\title{
Vibrational Thermodynamics of Materials
}

\author{
Brent Fultz \\ California Institute of Technology, W. M. Keck Laboratory, Pasadena CA 91125 USA
}

July 6,2009

\begin{abstract}
The literature on vibrational thermodynamics of materials is reviewed. The emphasis is on metals and alloys, especially on the progress over the last decade in understanding differences in the vibrational entropy of different alloy phases and phase transformations. Some results on carbides, nitrides, oxides, hydrides and lithium-storage materials are also covered.

Principles of harmonic phonons in alloys are organized into thermodynamic models for unmixing and ordering transformations on an Ising lattice, and extended for non-harmonic potentials. Owing to the high accuracy required for the phonon frequencies, quantitative predictions of vibrational entropy with analytical models prove elusive. Accurate tools for such calculations or measurements were challenging for many years, but are more accessible today. Ab-initio methods for calculating phonons in solids are summarized. The experimental techniques of calorimetry, inelastic neutron scattering, and inelastic x-ray scattering are explained with enough detail to show the issues of using these methods for investigations of vibrational thermodynamics. The explanations extend to methods of data analysis that affect the accuracy of thermodynamic information.

It is sometimes possible to identify the structural and chemical origins of the differences in vibrational entropy of materials, and the number of these assessments is growing. There has been considerable progress in our understanding of the vibrational entropy of mixing in solid solutions, compound formation from pure elements, chemical unmixing of alloys, order-disorder transformations, and martensitic transformations. Systematic trends are available for some of these phase transformations, although more examples are needed, and many results are less reliable at high temperatures. Nanostructures in materials can alter sufficiently the vibrational dynamics to affect thermodynamic stability. Internal stresses in polycrystals of anisotropic materials also contribute to the heat capacity. Lanthanides and actinides show a complex interplay of vibrational, electronic,
\end{abstract} and magnetic entropy, even at low temperatures.

A "quasiharmonic model" is often used to extend the systematics of harmonic phonons to high temperatures by accounting for the effects of thermal expansion against a bulk modulus. Non-harmonic effects beyond the quasiharmonic approximation originate from the interactions of thermally-excited phonons with other phonons, or with the interactions of phonons with electronic excitations. In the classical high temperature limit, the adiabatic electron-phonon coupling can have a surprisingly large effect in metals when temperature causes significant changes in the electron density near the Fermi level. There are useful similarities in how temperature, pressure, and composition alter the conduction electron screening and the interatomic force constants. Phononphonon "anharmonic" interactions arise from those non-harmonic parts of the interatomic potential that cannot be accounted for by the quasiharmonic model. Anharmonic shifts in phonon frequency with temperature can be substantial, but trends are not well understood. Anharmonic phonon damping does show systematic trends, however, at least for fcc metals.

Trends of vibrational entropy are often justified with atomic properties such as atomic size, electronegativity, electron-to-atom ratio, and mass. Since vibrational entropy originates at the level of electrons in solids, such rules of thumb prove no better than similar rules devised for trends in bonding and structure, and tend to be worse. Fortunately, the required tools for accurate experimental investigations of vibrational entropy have improved dramatically over the past few years, and the required ab-initio methods have become more accessible. Steady progress is expected for understanding the phenomena reviewed here, as investigations are performed with the new tools of experiment and theory, sometimes in integrated ways. 


\section{Table of Contents}

\section{Principles and Methods}

1 Overview 1

2 Harmonic Lattice Dynamics 2

2.1 Partition Function 3

2.2 Hamiltonian for Lattice Dynamics 5

2.3 Equations of Motion 6

2.4 The Eigenvalue Problem for the Polarization Vector 7

2.5 Calculation of the Phonon Density of States 8

3 Predictions with the Harmonic Model 9

3.1 Mass, Local Modes, and Resonance Modes 9

3.2 Long Wavelength Limit and the Debye Model 11

$\begin{array}{ll}3.3 & \text { Disordered Systems }\end{array}$

4 Bond Proportion Model 13

4.1 Bond Proportion Model and Unmixing on the Ising Lattice 14

$\begin{array}{lll}4.2 & \text { Bond Proportion Model and Ordering on the Ising Lattice }\end{array}$

4.3 Monte Carlo Results 20

5 Bond-Stiffness-versus-Bond-Length Model 21

5.1 Phonon Frequencies and Bond Lengths 21

5.2 Extending the Bond Proportion Model 24

5.3 Chemical Effects on Bond Stiffness 25

6 Heat Capacity 25

6.1 Harmonic Heat Capacity 25

$\begin{array}{ll}6.2 & \text { Quasiharmonic Thermodynamics } \\ & 27\end{array}$

6.3 Anharmonic Heat Capacity 29

6.4 Thermodynamic Entropy 30

7 Calorimeters 31

8 Neutron Scattering 32

8.1 Elastic and Inelastic Scattering 32

$\begin{array}{lll}8.2 & \text { One-Phonon and Multiphonon Scattering } & 35\end{array}$

$\begin{array}{lll}8.3 & \text { Neutron Weighting } & 37\end{array}$

8.4 Direct-Geometry Fermi Chopper Spectrometer 38

8.5 Triple-Axis Spectrometer 38

$9 \quad$ Ab-Initio Methods 39

9.1 Density Functional Theory 40

9.2 First-Principles Phonon Calculations 40

$\begin{array}{lll}9.3 & \text { Molecular Dynamics } & 41\end{array}$

II. Vibrational Entropy of Materials at Lower Temperatures

10 Earlier Investigations 43

10.1 Polymorphism 43

10.2 Solubilities and Defect Concentrations 44

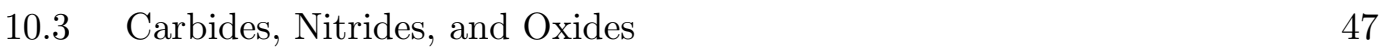

11 First-Principles Studies of Solutions and Studies of Ordered Compounds 48 
$\begin{array}{lll}11.1 & \text { Solubility in Al Alloys } & 48\end{array}$

$\begin{array}{lll}11.2 & \text { Aluminum Compounds } & 49\end{array}$

11.3 Low-Symmetry Phases at High Temperatures 50

12 Chemical Order-Disorder Transformations 52

12.1 Transition Metal Aluminides $\quad 52$

12.2 B2 Ordering in CuZn and FeCo 56

$\begin{array}{lll}12.3 & \mathrm{~L}_{2} \text { Ordering } & 57\end{array}$

13 Unmixing $\quad 62$

13.1 Computational Studies of Unmixing 62

13.2 Cluster Expansion Method for Solid Solutions 64

$\begin{array}{lll}13.3 & \text { Fe-Cr Solid Solutions } & 66\end{array}$

14 Interstitial Alloys $\quad 68$

$\begin{array}{lll}14.1 & \text { Interstitial Oxygen and Nitrogen } & 68\end{array}$

$\begin{array}{lll}14.2 & \text { Hydrogen and Hydrides } & 69\end{array}$

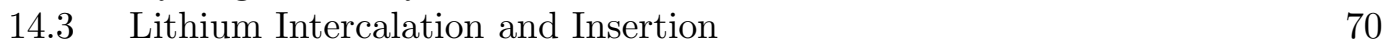

15 Martensite and Twinning $\quad 73$

16 Microstructural Contributions $\quad 76$

$\begin{array}{lll}16.1 & \text { Elastic Energy } & 77\end{array}$

$\begin{array}{lll}16.2 & \text { Nanostructures } & 79\end{array}$

17 f-Electron Metals 84

$\begin{array}{lll}17.1 & \text { Cerium } & 84\end{array}$

$\begin{array}{lll}17.2 & \text { Uranium and Plutonium } & 87\end{array}$

\section{Vibrational Entropy of Materials at Higher Temperatures}

18 Interactions between Thermal Excitations of Electrons and Phonons $\quad 89$

18.1 Overview of Thermal Effects on Electron and Phonon Energies 89

18.2 Formalism of Phonon-Phonon Interactions 90

18.3 Formalism of Electron-Phonon Interactions 93

19 Phonon-Phonon Anharmonicity $\quad 95$

19.1 Damped Harmonic Oscillator 95

$\begin{array}{ll}19.2 & \text { Perturbation Theory } \\ & 97\end{array}$

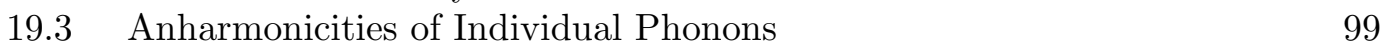

$\begin{array}{ll}19.4 & \text { Anharmonicity and Heat Capacity } \\ 101\end{array}$

19.5 Anharmonic Trends of fcc and bcc Metals 104

$\begin{array}{ll}19.6 & \text { Thermoelectric Materials } \\ & 107\end{array}$

20 Electron-Phonon Interactions in Metals 107

20.1 Fermi Surfaces 107

20.2 Phonons at High-Temperatures in Zirconium, Hafnium, Niobium, Molybdenum, and Chromium 110

20.3 Phonons in Vanadium and Its Alloys 110

20.4 The Adiabatic EPI in Superconductors 115

20.5 Effects of Pressure, Composition, and Temperature on the Adiabatic EPI and the Phonon DOS 118 


\section{Overview and Summary}

21 Systematic Trends of Vibrational Entropy 121

$\begin{array}{lll}21.1 & \text { Multiple Effects } & 121\end{array}$

21.2 Hume-Rothery Rules and Vibrational Entropy 122

22 Future Directions 123

$\begin{array}{lll}22.1 & \text { Opportunities for Experiment } & 123\end{array}$

$\begin{array}{lll}22.2 & \text { Opportunities for Theory } & 123\end{array}$

22.3 Outlook 124

23 Acknowledgments 125

24 Bibliography 125

$\begin{array}{ll}\text { Index } & 135\end{array}$ 


\section{Principles and Methods}

\section{Overview}

Without entropy to complement energy, thermodynamics would have the impact of one hand clapping. Boltzmann's graveside monument displays an equation for entropy of breathtaking generality

$$
S=k_{\mathrm{B}} \ln \Omega
$$

(modernized slightly, with $k_{\mathrm{B}}$ as the Boltzmann constant). The nub of the problem is the number $\Omega$, which counts the ways of finding the internal coordinates of a system for thermodynamically-equivalent macroscopic states. Physical questions are, "What do we count, and how do we count them?"

It is often possible to separate the internal coordinates of a material into configurational ones and dynamical ones. As an example, when the number $\Omega$ for configurational coordinates counts the spatial arrangements of atoms over the sites of a crystal, this $\Omega$ does not depend explicitly on temperature. On the other hand, when the number $\Omega$ for dynamical coordinates counts the intervals of volume explored as atoms vibrate, this $\Omega$ increases with temperature, as does the vibrational entropy.

Configurational entropies of atoms or spins undergo changes during chemical ordering or magnetic phase transformations, respectively. ${ }^{1}$ Configurational entropy was largely understood by Gibbs, who presented some of the combinatoric calculations of entropy that are used today [1]. The calculation of $\Omega$ is more difficult when there are local correlations in the system, but cluster approximation methods have proved powerful and accurate $[2,3,4]$. An important point is that configurational coordinates have different equilibrium values at different temperatures, but temperature does not alter how the configurations are counted to obtain $\Omega$.

Dynamical entropy grows larger as internal degrees of freedom of a solid, such as normal modes of vibration, become more strongly excited by thermal energy. ${ }^{2}$ With increasing temperature more phonons are created, and the vibrational excursions of atomic nuclei are larger. Fundamentally, the entropy from dynamical sources increases with temperature because with stronger excitations of dynamical degrees of freedom, the system explores a larger volume in the phase space of position and momentum. This volume, normalized if necessary, is the $\Omega$ for Eq. 1 .

Phonon thermodynamics, especially vibrational entropy, is the main focus of this review. It turns out that it is important to have rather accurate information on phonon energies or heat capacities to assess the importance of vibrational entropy. ${ }^{3}$

\footnotetext{
1 Electronic entropy can also have a configurational component in mixed-valent systems. Nuclear spins undergo ordering transitions at low temperatures, too, although at most temperatures of interest in materials physics the nuclear spins are fully disordered and their entropy does not change with temperature.

2 Temperature also drives electronic excitations to unoccupied states, and when many states are available the electronic entropy is large. Spin excitations are another source of entropy, but care must be taken when counting them if the configurations of spin disorder are already counted.

${ }^{3}$ For example, if we apply Eq. 16 to a case where the phonon density of states curves of the $\alpha$ and $\beta$ phases have the same shape, but differ in energy scaling by $10 \%$, we obtain a change in
} 
When comparing the phonon entropies of different alloy phases, a 1\% accuracy in the difference in logarithmic-averaged phonon energy is desireable. This is challenging, so although there were many suggestions over many years that vibrational entropy could have a significant effect on the thermodynamics of order-disorder transformations, until recently accurate computations of phonons were impractical, and the experimental results were ambiguous. Today, measurements and calculations have proved sufficiently reliable for useful assessments of the thermodynamic effects of vibrational entropy for different materials. This progress and the trends that have been found to date are the subjects of the present review.

The independence of phonons is an important feature of the "harmonic model," for which there are many useful and powerful mathematical results. The harmonic model explains many physical phenomena, especially at low temperatures when the phonons are approximately independent of each other, and independent of other sources of dynamical entropy. It is often extended to higher temperatures as the "quasiharmonic model," which assumes independent phonons with altered frequencies. At high temperatures, however, the different sources of dynamical entropy can interact in important ways. The existence of phonons alters the energies required to create additional phonons (phonon-phonon interaction, PPI), and the electron excitations are altered by vibrational excitations (electron-phonon interaction, EPI). An understanding of vibrational entropy at high temperatures is emerging.

The first sections of this review develop the principles of phonon thermodynamics and vibrational entropy. Methods for measurement and calculation are covered next, with emphasis on experimental methods. The third part is on the vibrational entropies of materials with different atomic configurations, and phase transformations, emphasizing low temperature behavior interpreted with a harmonic model. The fourth part is on non-harmonic behavior at higher temperatures, including the effects of electron-phonon and phonon-phonon interactions.

\section{Harmonic Lattice Dynamics}

The mass of an atom is in its point-like nucleus, and the forces between nuclei are transmitted through lightweight electrons, which are typically assumed to respond instantly to nuclear displacements (Born-Oppenheimer approximation). The Bornvon Kármán model assumes nuclear masses connected by massless electron springs, giving a set of ordinary second-order differential equations of motion. The model is specific to a crystal with translational periodicity, and its normal modes of vibration are wavelike and with dispersion relations between frequency and wavevector. The Born-von Kármán model is expected to be reliable at low temperatures where atom displacements are small.

Normal coordinates offer a sweeping simplification of the vibrational dynamics. In a harmonic solid, each normal mode does not transfer energy to the other modes. Without damping, a normal mode persists forever. This remains true in quantum mechanics, although integral numbers of excitations, phonons, exist in each normal

phonon entropy of $\Delta S_{\mathrm{ph}}=3 k_{\mathrm{B}} \ln (1.1) \simeq 0.3 k_{\mathrm{B}} /$ atom. This change in vibrational entropy is almost half of the maximum possible change in configurational entropy of an order-disorder transformation of a binary alloy (which is $k_{\mathrm{B}} \ln 2 /$ atom). 
mode. For harmonic solids, the energy cost for each additional phonon is $\varepsilon_{i}=$ $\hbar \omega_{i}$ ( $\omega_{i}$ is the angular frequency of the oscillator), and is unchanged even as more phonons are added to each normal mode. Phonon modes can therefore be considered as independent harmonic oscillators, so we start with the thermodynamics of the quantum harmonic oscillator.

\subsection{Partition Function}

The partition function for a single harmonic oscillator of frequency $\omega_{i}=\varepsilon_{i} / \hbar$ is

$$
\begin{aligned}
& Z_{i}=\sum_{n}^{\infty} \mathrm{e}^{-\beta(n+1 / 2) \varepsilon_{i}}, \\
& Z_{i}=\frac{\mathrm{e}^{-\beta \varepsilon_{i} / 2}}{1-\mathrm{e}^{-\beta \varepsilon_{i}}},
\end{aligned}
$$

where Eq. 3 was obtained by identifying Eq. 2 as a geometric series times the constant factor $\exp \left(-\beta \varepsilon_{i} / 2\right)$, where $\beta \equiv\left(k_{\mathrm{B}} T\right)^{-1}$. The partition function for a harmonic solid with $N$ atoms and $3 N$ independent oscillators is the product of these individual oscillator partition functions,

$$
Z_{N}=\prod_{i}^{3 N} \frac{\mathrm{e}^{-\beta \varepsilon_{i} / 2}}{1-\mathrm{e}^{-\beta \varepsilon_{i}}},
$$

from which we can calculate the phonon free energy by the prescription $F=$ $-k_{\mathrm{B}} T \ln Z$,

$$
F_{\mathrm{vib}}=\frac{1}{2} \sum_{i}^{3 N} \varepsilon_{i}+k_{\mathrm{B}} T \sum_{i}^{3 N} \ln \left(1-\mathrm{e}^{-\beta \varepsilon_{i}}\right),
$$

and the phonon entropy (vibrational entropy) by differentiating with respect to $T$ :

$$
S_{\mathrm{vib}}=k_{\mathrm{B}} \sum_{i}^{3 N}\left[-\ln \left(1-\mathrm{e}^{-\beta \varepsilon_{i}}\right)+\frac{\beta \varepsilon_{i}}{\mathrm{e}^{\beta \varepsilon_{i}}-1}\right] .
$$

Using the Planck distribution for phonon occupancy versus temperature, $n\left(\varepsilon_{i}, T\right)$,

$$
\begin{aligned}
n\left(\varepsilon_{i}, T\right) & =\frac{1}{\mathrm{e}^{+\beta \varepsilon_{i}}-1}, \\
-\ln \left(\mathrm{e}^{-\beta \varepsilon_{i}}-1\right) & =\ln (1+n), \\
\beta \varepsilon_{i} & =\ln \left(\frac{1+n}{n}\right),
\end{aligned}
$$

it is straightforward to show that Eq. 6 reduces to the following form for the vibrational entropy per oscillator, $S_{\text {osc }}$

$$
S_{\mathrm{osc}}=+k_{\mathrm{B}}[(1+n) \ln (1+n)-n \ln n] .
$$

Equation 10 can also be obtained from Eq. 1 with the combinatoric relationship $\Omega=(M+m) ! /[M ! m !]$ for $m$ phonons in $M$ oscillators (with $n \equiv m / M$ ). 
Phonons are bosons, so it is interesting to compare Eq. 10 to the analogous equation for fermions, where $m$ electrons are distributed over $N$ states of energy $\varepsilon$. For fermions, the combinatorics give $\Omega=N ! /[(N-m) ! m !]$. Fermion entropy is the same as the entropy of mixing of atoms on a crystal lattice, where each lattice site can hold only one atom. The entropy of mixing per atom is

$$
S_{\text {mix }}=-k_{\mathrm{B}}[(1-c) \ln (1-c)+c \ln c] .
$$

Equations 10 and 11 have two differences - all signs are reversed, and $n$ can exceed 1 , whereas $0 \leq c \leq 1$.

It is often useful to work with a phonon density of states (DOS), $g(\varepsilon)$. For a 3dimensional solid, $3 N g(\varepsilon) \mathrm{d} \varepsilon$ phonon modes are in an energy interval $\mathrm{d} \varepsilon$. For a DOS acquired as digital data in $m$ intervals of width $\Delta \varepsilon$ (so $\varepsilon_{j}=j \Delta \varepsilon$ ), the partition function can be computed numerically

$$
Z_{N}=\prod_{j=1}^{m}\left(\frac{\mathrm{e}^{-\beta \varepsilon_{j} / 2}}{1-\mathrm{e}^{-\beta \varepsilon_{j}}}\right)^{3 N g\left(\varepsilon_{j}\right) \Delta \varepsilon}
$$

which becomes at high temperatures

$$
Z_{N}=\prod_{j=1}^{m}\left(\frac{k_{\mathrm{B}} T}{\varepsilon_{j}}\right)^{3 N g\left(\varepsilon_{j}\right) \Delta \varepsilon} .
$$

Again, using Eqs. 7, 8, 9, we can obtain from Eq. 12 a useful expression for the phonon entropy of a harmonic material at any temperature (cf. Eq. 10)

$$
S_{\mathrm{vib}}(T)=3 k_{\mathrm{B}} \int_{0}^{\infty} g(\varepsilon)[(n(\varepsilon)+1) \ln (n(\varepsilon)+1)-n(\varepsilon) \ln (n(\varepsilon))] \mathrm{d} \varepsilon,
$$

where $g(\varepsilon)$ is normalized to 1 and $n(\varepsilon)$ is the Planck distribution (Eq. 7) for the temperature of interest. For thermodynamic calculations, series expansions of Eqs. 5 and 6 , valid at high temperatures, can be useful. For $S_{\mathrm{ph}}(T)$, Fig. 1 compares the exact expression of Eq. 10 to approximations for up to four terms of a standard hightemperature expansion (terms are presented in the figure). At high temperatures, the following expression works well for the entropy of one oscillator mode with energy $\varepsilon=\hbar \omega$

$$
S_{\mathrm{vib}, \omega}(T)=k_{\mathrm{B}}[(n(\varepsilon)+1) \ln (n(\varepsilon)+1)-n(\varepsilon) \ln (n(\varepsilon))] \simeq k_{\mathrm{B}}\left[\ln \left(\frac{k_{\mathrm{B}} T}{\hbar \omega}\right)+1\right],
$$

although the constant factor of $k_{\mathrm{B}}$ will cancel in differences of phonon entropy. A handy expression for the high-temperature limit of the difference in vibrational entropy between two harmonic phases, $\alpha$ and $\beta$, can be obtained readily from Eq. 14

$$
\Delta S_{\mathrm{vib}}^{\beta-\alpha}=3 k_{\mathrm{B}} \int_{0}^{\infty}\left(g^{\alpha}(\varepsilon)-g^{\beta}(\varepsilon)\right) \ln (\varepsilon) \mathrm{d} \varepsilon
$$




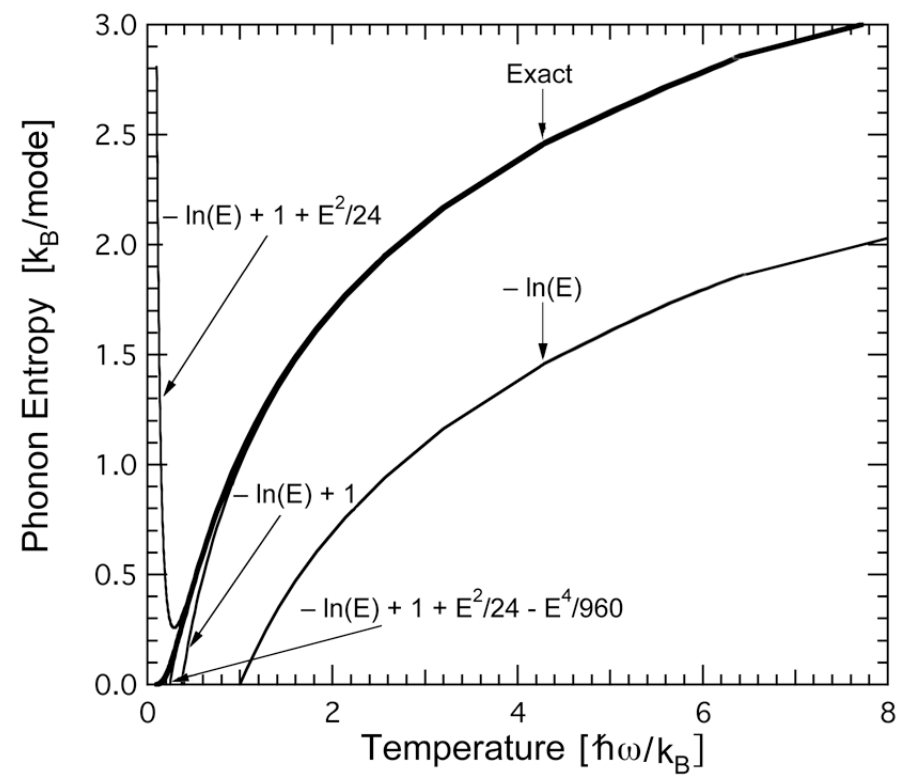

Figure 1. Temperature-dependence of entropy calculated for one mode of energy $\hbar \omega$, with $E=\hbar \omega\left(k_{\mathrm{B}} T\right)^{-1}$.

The important point about Eq. 12 is that the only material parameter relevant to the thermodynamic partition function in the harmonic approximation is the phonon DOS, $g(\varepsilon)$. Measuring or calculating the phonon DOS is central to much of our understanding of the vibrational entropy of materials. It turns out, though, that for most problems $g(\varepsilon)$ must be known with high accuracy, and this has been a challenge until relatively recently.

\subsection{Hamiltonian for Lattice Dynamics}

Since most of the atom mass is in the nucleus, for lattice dynamics we consider the Hamiltonian for nuclear motions in a crystal

$$
H_{\mathrm{n}}=\sum_{l, \kappa} \frac{\vec{p}_{l \kappa}^{2}}{2 m_{\kappa}}+\Phi,
$$

and the characteristics of the atom vibrations require attention to the potential energy, $\Phi$. Following the development of Maradudin, et al. [5] for a crystal lattice with a basis, the basis vectors are $\left\{\vec{r}_{\kappa}, \kappa=1,2 \ldots \mathcal{R}\right\}$. Each atom $\kappa$, in the unit cell $l$, is allowed to vibrate about equilibrium with the displacement $\vec{u}_{l \kappa}(t)$. The instantaneous position $\vec{R}_{l \kappa}(t)$ of atom $l \kappa$ at time $t$ is

$$
\vec{R}_{l \kappa}(t)=\vec{r}_{l}+\vec{r}_{\kappa}+\vec{u}_{l \kappa}(t) .
$$

We use Cartesian components $u_{\alpha l \kappa}$ for the displacement vector, where $\alpha=\{x, y, z\}$. The total potential energy of the crystal, $\Phi$, is a function of the instantaneous positions of all the atoms in the crystal, but it is expanded in a Taylor series of the atomic displacements about $\vec{u}_{l \kappa}(t)=0$ (i.e., the equilibrium positions of the atoms 
$\left.\vec{R}_{l \kappa}=\vec{r}_{l}+\vec{r}_{\kappa}\right)$

$$
\begin{aligned}
\Phi=\Phi_{0} & +\sum_{\alpha l \kappa} \Phi_{\alpha l \kappa} u_{\alpha l \kappa} \\
& +\frac{1}{2} \sum_{\alpha l \kappa} \sum_{\alpha^{\prime} l^{\prime} \kappa^{\prime}} \Phi_{\alpha \alpha^{\prime} l \kappa l^{\prime} \kappa^{\prime}} u_{\alpha l \kappa} u_{\alpha^{\prime} l^{\prime} \kappa^{\prime}}+\ldots,
\end{aligned}
$$

where the coefficients of the Taylor series are the derivatives of the potential with respect to the displacements:

$$
\begin{aligned}
\Phi_{\alpha l \kappa} & \left.=\frac{\partial \Phi}{\partial u_{\alpha l \kappa}}\right)_{0}, \\
\Phi_{\alpha \alpha^{\prime} l \kappa l^{\prime} \kappa^{\prime}} & \left.=\frac{\partial^{2} \Phi}{\partial u_{\alpha l \kappa} \partial u_{\alpha^{\prime} l^{\prime} \kappa^{\prime}}}\right)_{0},
\end{aligned}
$$

where the subscript zero means that derivatives are evaluated in the equilibrium configuration (all displacements equal to zero) and $\Phi_{0}$ is the static potential energy of the crystal. Because the force on any atom must vanish in the equilibrium configuration, we have [5]

$$
\Phi_{\alpha l \kappa}=0 \quad \forall \alpha, l, \kappa .
$$

In the harmonic approximation of lattice dynamics we keep only the remaining terms of the series written explicitly in Eq. 19 - we neglect terms of order three and higher in the displacements

$$
H_{\mathrm{n}}=\sum_{l \kappa} \frac{\vec{p}_{l \kappa}^{2}}{2 m_{\kappa}}+\Phi_{0}+\frac{1}{2} \sum_{\alpha l \kappa} \sum_{\alpha^{\prime} l^{\prime} \kappa^{\prime}} \Phi_{\alpha \alpha^{\prime} l \kappa l^{\prime} \kappa^{\prime}} u_{\alpha l \kappa} u_{\alpha^{\prime} l^{\prime} \kappa^{\prime}}
$$

where $m_{\kappa}$ is the mass of the atom at basis index $\kappa$ of the unit cell. We rewrite the Hamiltonian in matrix form

$$
H_{\mathrm{n}}=\sum_{l \kappa} \frac{\vec{p}_{l \kappa}^{2}}{2 m_{\kappa}}+\Phi_{0}+\frac{1}{2} \sum_{l \kappa} \sum_{l^{\prime} \kappa^{\prime}} \vec{u}_{l \kappa}^{T} \underline{\Phi}_{l \kappa l^{\prime} \kappa^{\prime}} \vec{u}_{l^{\prime} \kappa^{\prime}}
$$

where a $3 \times 3$ force-constant sub-matrix is defined for each atom pair $\left(l \kappa ; l^{\prime} \kappa^{\prime}\right)$

$$
\underline{\Phi}_{l \kappa l^{\prime} \kappa^{\prime}}=\left[\Phi_{\alpha \alpha^{\prime} l \kappa l^{\prime} \kappa^{\prime}}\right] \text {. }
$$

If $(l, \kappa) \neq\left(l^{\prime}, \kappa^{\prime}\right)$, Eq. 21 applies. If $(l, \kappa)=\left(l^{\prime}, \kappa^{\prime}\right), \Phi_{\alpha \alpha^{\prime} l \kappa l \kappa}$ is a "self-force constant," derived from the requirement of no overall translation of the crystal

$$
\underline{\Phi}_{l \kappa l \kappa}=-\sum_{\left(l^{\prime}, \kappa^{\prime}\right) \neq(l, \kappa)} \underline{\Phi}_{l \kappa l^{\prime} \kappa^{\prime}}
$$

Because equal and opposite forces act between each atom of a pair, $\Phi_{l \kappa l^{\prime} \kappa^{\prime}}$ must be a real symmetric matrix. 


\subsection{Equations of Motion}

In the harmonic approximation, the equations of motion for all nuclei are

$$
m_{\kappa} \ddot{\vec{u}}_{l \kappa}(t)=-\sum_{l^{\prime}, \kappa^{\prime}} \underline{\Phi}_{l \kappa l^{\prime} \kappa^{\prime}} \vec{u}_{l^{\prime} \kappa^{\prime}}(t) \quad \forall l, \kappa .
$$

In Eq. 27 there are $3 \times \mathcal{R} \times N^{\text {cell }}$ equations of motion to solve for a finite crystal containing $N^{\text {cell }}$ unit cells. We seek solutions having the form of plane waves ${ }^{4}$ of wavevector $\vec{k}$, angular frequency $\omega_{\vec{k} j}$, and "polarization" $\vec{e}_{\kappa j}(\vec{k})$ (where $j$ is a "branch index" discussed below)

$$
\begin{aligned}
& \vec{u}_{l \kappa \vec{k} j}(t)=\sqrt{\frac{2 \hbar}{N m_{\kappa} \omega_{\vec{k} j}}} \vec{e}_{\kappa j}(\vec{k}) \mathrm{e}^{\mathrm{i}\left(\vec{k} \cdot \vec{r}_{l}-\omega_{\vec{k} j} t\right)} . \\
& \vec{u}_{l \kappa \vec{k} j}(t)=\hbar \sqrt{\frac{2 n\left(\varepsilon_{\vec{k} j}, T\right)+1}{N m_{\kappa} \varepsilon_{\vec{k} j}}} \vec{e}_{\kappa j}(\vec{k}) \mathrm{e}^{\mathrm{i}\left(\vec{k} \cdot \vec{r}_{l}-\omega_{\vec{k} j} t\right)},
\end{aligned}
$$

where we take the real part to obtain physical displacements, or we use the convention of adding the complex conjugate of the exponential, and halving the prefactor. The phase factor, $\mathrm{e}^{\mathrm{i} \vec{k} \cdot \vec{r}_{l}}$, provides all the long-range spatial modulation of $\vec{u}_{l \kappa \vec{k} j}(t)$. The dependence on $\kappa$, a short-range basis vector index, is taken out of the phase factor and placed in the complex constant $\vec{e}_{\kappa j}(\vec{k})$. It is convenient for the $\vec{e}_{\kappa j}(\vec{k})$ of Eq. 28 to have modulus unity, as does the exponential. The prefactors are consistent with the quantization of energy for one phonon, $\hbar \omega=m \omega^{2}\left\langle u^{2}\right\rangle=\frac{1}{2} m \omega^{2} u_{\max }^{2}$, or for the thermal population of phonons in mode $\varepsilon_{\vec{k} j}$, having energy $E=\varepsilon_{\vec{k} j}\left[n\left(\varepsilon_{\vec{k} j}, T\right)+\frac{1}{2}\right]$. (For root-mean-squared displacements, delete the $\sqrt{2}$ in Eqs. 28 and 29.)

We impose periodic boundary conditions on the finite crystal. These require the set of possible wavevectors $\{\vec{k}\}$ to have $N^{\text {cell }}$ values, a large number that gives a very fine mesh of $\vec{k}$-points in reciprocal space. With $\mathcal{R}$ atoms in the basis of the unit cell, the crystal has $3 \times \mathcal{R} \times N^{\text {cell }}$ vibrational modes, in agreement with its total number of mechanical degrees of freedom. Each wavevector $\vec{k}$ is associated a-priori with $3 \times \mathcal{R}$ types of vibrational modes, identified by a branch index, $j$. Each of the $3 \mathcal{R}$ different modes corresponds to a different polarization vector $\vec{e}_{\kappa j}(\vec{k})$ and angular frequency $\omega_{\vec{k} j}(1 \leq j \leq 3 \mathcal{R})$, although degeneracies can be induced by symmetry.

\subsection{The Eigenvalue Problem for the Polarization Vector}

The polarization vector, $\vec{e}_{\kappa j}(\vec{k})$, is a characteristic of each vibrational mode $\vec{k}, j$. The vector $\vec{e}_{\kappa j}(\vec{k})$ contains information on the excursion of each atom $\kappa$ in the unit cell for the phonon mode mode $\vec{k}, j$. Specifically, it gives 1) the displacement direction of the atom, and 2) its phase lag in time with respect to the other atoms. The vectors $\vec{e}_{\kappa j}(\vec{k})$ for all the atoms in the basis $(1 \leq \kappa \leq \mathcal{R})$ and their associated

\footnotetext{
${ }^{4}$ In general, the motion of an atom $\vec{u}_{l \kappa}(t)$ will be a sum over many phonons $\vec{u}_{l \kappa \vec{k} j}(t)$ (cf., Eq. $143)$.
} 
angular frequencies $\omega_{\vec{k} j}$ can be calculated by diagonalizing the "dynamical matrix" $\underline{D}(\vec{k})$. The dynamical matrix is obtained by substituting Eq. 28 into 27 . It has the dimensions $(3 N \times 3 N)$ and is constructed from $(3 \times 3)$ submatrices $\underline{D}_{\kappa \kappa^{\prime}}(\vec{k})$

$$
\underline{D}(\vec{k})=\left(\begin{array}{ccc}
\underline{D}_{11}(\vec{k}) & \cdots & \underline{D}_{1 N}(\vec{k}) \\
\vdots & \ddots & \vdots \\
\underline{D}_{N 1}(\vec{k}) & \cdots & \underline{D}_{N N}(\vec{k})
\end{array}\right) .
$$

Each sub-matrix $\underline{D}_{\kappa \kappa^{\prime}}(\vec{k})$ is the Fourier transform of the force-constant matrix $\underline{\Phi}_{l \kappa l^{\prime} \kappa^{\prime}}$, considered as a function of $\left(l^{\prime}-l\right)$ :

$$
\underline{D}_{\kappa \kappa^{\prime}}(\vec{k})=\frac{1}{\sqrt{m_{\kappa} m_{\kappa^{\prime}}}} \sum_{l^{\prime}} \underline{\Phi}_{0 \kappa l^{\prime} \kappa^{\prime}} \mathrm{e}^{i \vec{k} \cdot\left(\vec{r}_{l^{\prime}}-\vec{r}_{0}\right)},
$$

where we set $l=0$ because the summation is over all $l^{\prime}$ and the origin is arbitrary. By similarly collecting the polarization vectors into a vector of size $3 \times \mathcal{R}$, we rewrite the system of differential equations (Eq. 27) with the plane wave solutions (Eq. 28) as an eigenvalue problem:

$$
\underline{D}(\vec{k}) \vec{e}_{j}(\vec{k})=\omega_{\vec{k} j}^{2} \vec{e}_{j}(\vec{k})
$$

where

$$
\vec{e}_{j}(\vec{k})=\left(\begin{array}{c}
e_{x 1 j}(\vec{k}) \\
e_{y 1 j}(\vec{k}) \\
e_{z 1 j}(\vec{k}) \\
e_{x 2 j}(\vec{k}) \\
\vdots \\
e_{z N j}(\vec{k})
\end{array}\right)
$$

It can be shown that the $(3 \mathcal{R} \times 3 \mathcal{R})$ dynamical matrix $\underline{D}(\vec{k})$ is hermitian (for any value of $\vec{k}$ ). It is fully diagonalizable, and the $\omega_{\vec{k} j}^{2}$ are real. The $3 \mathcal{R}$ eigenvectors and eigenvalues of the dynamical matrix evaluated at a particular wavevector $\vec{k}$ then correspond to the $3 \mathcal{R}$ eigenmodes of vibration of the crystal for that wavevector.

\subsection{Calculation of the Phonon Density of States}

To calculate the phonon density of states (DOS) of the crystal, $g(\varepsilon)$, the dynamical matrix is diagonalized at a large number of points in reciprocal space (typically covering the first Brillouin zone). The diagonalization of $\underline{D}(\vec{k})$ at each $\vec{k}$ point returns $3 \mathcal{R}$ eigenvalues of angular frequency $\omega_{\vec{k} j}(1 \leq j \leq 3 \mathcal{R})$, which are then binned into the DOS histogram.

A phonon partial DOS, $g_{d}(\varepsilon)$, is a similar quantity, but it gives the spectral distribution of motion by one atom, the species $d$ in the unit cell. Unlike the total DOS, $g(\varepsilon)$, the eigenvalues of the crystal are not weighted equally in the partial 
DOS

$$
g_{d}(\varepsilon)=\sum_{\vec{k}} \sum_{\alpha \kappa j} \delta_{d \kappa}\left|e_{\alpha \kappa j}(\vec{k})\right|^{2} g(\varepsilon),
$$

where the Kroneker delta is zero unless the atom species $d$ is at the site $\kappa$ (when $\delta_{d d}=1$ ). Because the eigenvalues of the dynamical matrix are normalized for each $\vec{k}$ as

$$
\sum_{\alpha \kappa j}\left|e_{\alpha \kappa j}(\vec{k})\right|^{2}=1
$$

the total DOS is the sum of the partial DOSs of all atoms in the unit cell,

$$
g(\varepsilon)=\sum_{d} g_{d}(\varepsilon)
$$

To calculate a partial DOS, for each diagonalization of the dynamical matrix at a specific $\vec{k}$ point, the partial DOS histogram for atom $d$ is incremented at frequency $\omega_{\vec{k} j}$ by the amount $\sum_{\alpha} e_{\alpha d j}^{*}(\vec{k}) e_{\alpha d j}(\vec{k})$. The partial DOS for atom $d$ is large at energies where there are many modes with large displacements of atom $d$. The histogram for the phonon partial DOSs are computed simultaneously with the total DOS.

\section{Predictions with the Harmonic Model}

The harmonic model provides much of the truth about vibrational entropy at low temperatures, and its analytical formulation allows a number of important predictions about vibrational entropies of alloying or phase transformations. Some important results are presented here without full proof. Two classic and sophisticated treatments of the harmonic solid are Maradudin, et al. [5], and Born and Huang [6].

\subsection{Mass, Local Modes, and Resonance Modes}

Otto Stern reported a number of theoretical observations relevant to lattice dynamics in 1916 [7]. Importantly, he showed that the trace of the dynamical matrix is equal to the product of the normal mode frequencies squared. From this it is possible to show that mass disorder does not contribute to the vibrational entropy of orderdisorder or unmixing transformations, for example. If a new structure is formed by rearranging the same atoms, the effects of the masses on the vibrational entropy can be neglected, at least in the classical limit. All changes in vibrational entropy are attributed to changes in force constants (or changes in the interatomic potential), and not to rearranging the same masses $[8,9,10,11]$

The kinetic energy for each atom is $p^{2} /(2 m)$, where the momentum, $p$, is a maximum when the atom is at its equilibrium position $\left(\vec{u}_{l_{\kappa}}=0\right)$ and all the thermal energy is kinetic. Rearranging the different atom masses into different structures has no effect on the kinetic energy of each atom as it moves across its equilibrium position. There is no change in the volume of momentum space explored during 
vibration. The potential energy of each oscillating atom does not depend on mass, only position. Changing the mass of an atom will not change the range of its motion in the potential well $\left(\operatorname{Max}\left[\vec{u}_{l \kappa}(t)\right]\right)$, or the volume of phase space in the position coordinates. Since the volume of momentum-position phase space is unchanged by rearranging the same masses, by Eq. 1 there is no change in vibrational entropy in the classical limit. Only changes in interatomic force constants are important when comparing classical vibrational entropies of different phases in the harmonic model. Anharmonicity does not change this independence of mass, but it alters other results. ${ }^{5}$

The Einstein model, which assumes each atom is an independent harmonic oscillator, cannot account for wavevector-energy relationships of phonons. Nevertheless, the Einstein model can be useful when little energy is transferred between the atoms in the solid, and there is little change of phonon energy with wavevector. Consider hydrogen atoms in interstitial sites between metal atoms. Owing to its low mass, a hydrogen atom vibrates as if in a cage. Although the neighboring metal atoms must move a bit in response to the moving hydrogen atom, these metal atom displacements are small. If the interatomic forces between the metal and hydrogen atoms are moderate, the system will show a "local mode" at a high frequency, typically above the modes of the metal crystal. The local mode is dominated by motions of the hydrogen atoms, although the metal atoms must have some contribution in their own partial density of states.

The other extreme of a heavy impurity atom in a host of lighter atoms produces a "resonance mode," where the partial phonon DOS of the heavy impurity is dominated by a peaked spectrum at low energy. The neighboring matrix atoms move in response to the local motions of the heavy impurity atom, but the dynamics is not simple. Because the resonance mode exists at the same frequency as other modes in the matrix, there is substantial coupling between the resonance mode and the continuum, so the resonance mode is not an eigenmode of a harmonic solid. A resonance mode appears as being highly damped, with a short lifetime and a broad width in energy, sometimes of the form [5]

$$
\Delta g(\omega) \propto \frac{\Gamma}{\left(\omega-\omega_{0}\right)^{2}+\Gamma^{2}}
$$

The resonance mode is not intuitive, and its discovery was an achievement of theoretical work $[12,13,14,15]$.

The existence of a resonance mode depends on the ratios of the masses and interatomic force constants. If all interatomic force constants are equal, a resonance mode will exist in an fcc lattice if the impurity mass is about 2.6 times heavier than the atoms in the matrix [5]. More likely, the interatomic force constants will differ between the host atoms and the impurity, compared to host-host force constants. Stiffer forces to the neighbors of the impurity effectively increase the mass of the defect center, favoring a resonance mode.

\footnotetext{
${ }^{5}$ For harmonic oscillators, the thermal energy is equipartitioned between kinetic and potential energy. The equipartition of $k_{\mathrm{B}} T / 2$ into each degree of freedom of momentum and position originates with these coordinates appearing quadratically in the Hamiltonian $H$, producing a Gaussian integration of $\mathrm{e}^{-H / k_{\mathrm{B}} T}$. A cubic or quartic term in $H$ causes an imbalance between kinetic and potential energy.
} 


\subsection{Long Wavelength Limit and the Debye Model}

The relative motions of different atoms in the unit cell are easiest to understand in the long wavelength limit. Each inequivalent atom in a unit cell forms a sublattice connecting its equivalent atoms in all other unit cells, distinct from the sublattices of the other atoms. In the limit of long waves (as the wavevector $k \rightarrow 0$ ), these sublattices move rigidly, as seen on the scale of many unit cells. In optical modes, the sublattices move against each other, out-of-phase for example, but equivalent atoms in adjacent unit cells have the same displacements at the same time. In the acoustic modes, the motions of all sublattices are the same, as adjacent unit cells distort their shapes in unison.

Acoustic modes at long wavelengths are elastic waves through solids. The anisotropy of a crystalline solid adds complexity to the problem, but consider the wave equation for the propagation of a longitudinal wave along the [100] direction:

$$
\omega^{2} \rho=C_{11} k_{[100]},
$$

which has a wave speed of $v_{1,[100]}=\sqrt{C_{11} / \rho}\left(C_{11}\right.$ is an elastic constant and $\rho$ is density). This wave speed $v_{1,[100]}$ must equal the group velocity obtained from the longitudinal branch of the phonon dispersion along [100] at small $k$ :

$$
\underset{k_{[100]} \rightarrow 0}{\mathcal{L i m}} \mathrm{d} \omega / \mathrm{d} k=\sqrt{C_{11} / \rho} .
$$

Relationships like Eqs. 38 and 39 hold true in all directions, although different combinations of elastic constants are required. Equation 39 requires a set of relationships between the elastic constants (such as $C_{11}$ ) and the interatomic force constants $\Phi_{l k l^{\prime} k^{\prime}}$, which determine the phonon dispersions through Eqs. 31 and 32. Physically, the elastic constants originate from the interatomic forces, so such a relationship is expected. The derivations of such relationships are not simple, however, owing to the anisotropy of the interatomic forces and differences between crystal structures.

Sound velocities are the rigorous limit of $\mathrm{d} \omega / \mathrm{d} k$ as $k \rightarrow 0$. At the atomic scale, a long wavelength longitudinal phonon is a density wave of compression followed by tension. In a local region (much smaller than $\lambda$ ), all atoms move collectively towards and against their neighbors, irrespective of their mass or interatomic force constants. When $\lambda$ exceeds the range of the interatomic forces, the wave propagation becomes independent of atomic detail, and in a given direction all waves of the same polarization propagate with the same speed. Continuum behavior is achieved.

A subtlety arises when anharmonicity is significant, such as at high temperatures. Anharmonicity can suppress phonon lifetimes to tens of vibrational periods or less (Sect. 19.5). In this case, the long acoustic waves measured by ultrasonics propagate through a medium influenced by averaged anharmonic effects. On the other hand, the acoustic phonons measured by neutron inelastic scattering propagate in their reduced lifetimes across a medium where equilibration of vibrational energy is not taking place between phonon modes. With anharmonicity, the energies and $\mathrm{d} \omega / \mathrm{d} k$ of the neutron measurements are often larger than expected from ultrasonic measurements.

The Debye model of phonons in solids extrapolates this long wavelength behavior to all phonon frequencies, $\omega$, by assuming they are linear functions of wavevector, $k \equiv$ 
$2 \pi / \lambda$. This assumption never works for optical phonons, and is erroneous for acoustic waves when $k$ approaches a reciprocal lattice vector (i.e., $\lambda$ is interatomic distances). In particular, the highest phonon frequency occurs when nearest-neighbor atoms move out-of-phase with respect to each other. Here there is no wave propagation, and $\mathrm{d} \omega / \mathrm{d} k=0$.

Comparing the Debye temperatures of phases before and after a phase transformation allows an estimate of the change in vibrational entropy, using for example a high temperature expression like $\Delta S^{\alpha \rightarrow \beta}=3 k_{\mathrm{B}} \ln \left(\Theta_{\mathrm{D} \alpha} / \Theta_{\mathrm{D} \beta}\right)$, where $\Theta_{\mathrm{D} \alpha}$ and $\Theta_{\mathrm{D} \beta}$ are Debye temperatures of phases $\alpha$ and $\beta$. This must be done with care, however, because the Debye model has a history of misleading the materials physics community about the importance of vibrational entropy. The Debye model is unreliable for any calculations of vibrational entropy when the Debye temperature is obtained from experimental measurements of long wavelength behavior such as sound velocities, because only the low-energy tail of the phonon DOS is sampled. Differences in this tail usually do not correlate reliably with differences in the phonon spectra of different materials because most of the phonons have wavelengths of atomic distances. On the other hand, Debye temperatures obtained from heat capacity measurements are more representative of the phonon contributions to the entropy, and are more trustworthy for estimates of vibrational entropy. Beware that the Debye temperature is sometimes graphed as a function of temperature, which is a confession that the high frequency phonons cannot be obtained reliably from long-wavelength vibrations.

Fernández Guillermet and Grimvall tested modifications of the Debye temperature for evaluating vibrational entropy and phonon spectra [16]. Specifically they defined a Debye temperature from the standard entropy of pure elements, as obtained for example from the JANAF Thermochemical Tables, with a small correction for electronic entropy. A second Debye temperature was obtained from bulk moduli, but it differed substantially from that for the heat capacity. For $\mathrm{Ru}$, for example, the two Debye temperatures were 384 and $555 \mathrm{~K}$, and the effective interatomic force constants derived from the two Debye temperatures differed by a factor of two. ${ }^{6}$ For $3 d$, $4 d$, and $5 d$ elements in the same column of the periodic table, there are fairly constant ratios of the two types of force constants. Nevertheless, the large disparities between Debye temperatures obtained from the entropy and from the elastic constants do not bode well for determining vibrational entropy from Debye temperatures. For some bcc and hcp transition metals, the ratios of Debye temperatures deduced from the entropy at ambient temperature and at cryogenic temperature range from 0.8 to 1.0 [17]. This indicates that the Debye temperature useful for determining differences in vibrational entropy is obtained from the vibrational entropy at the temperature of interest, i.e., the Debye temperature should be a recasting of the vibrational entropy itself.

It is possible to account for the effects of thermal expansion on the Debye temperature with a Debye-Grüneisen approximation, where $\Theta_{\mathrm{D}}(T)=\Theta_{\mathrm{D}}^{0}\left[r_{0} / r(T)\right]^{3 \gamma}$. With increasing $T, \Theta_{\mathrm{D}}(T)$ becomes smaller as $r(T)$ becomes larger. This approach was used by Moruzzi, et al. to calculate thermal properties of elements [18]. Sanchez, et al., used this approach to improve the effective cluster interactions for first-principles

\footnotetext{
${ }^{6}$ The force constant, $k=M \omega^{2}$, and with $\hbar \omega=k_{\mathrm{B}} \Theta_{\mathrm{D}}, k=M\left(k_{\mathrm{B}} \Theta_{\mathrm{D}} / \hbar\right)^{2}$.
} 
phase diagram calculations [19]. Tseng and Stark used this Debye-Grüneisen approximation to calculate the solubility of $\mathrm{Ti}$ in $\mathrm{Al}$ at both elevated temperatures, and under pressure [20].

\subsection{Disordered Systems}

The pioneering analytical work on the disordered two-component chain by Dyson [21] inspired a number of studies on the dynamics of disordered systems. This field is excellent for computational methods, and the value of computer calculations of lattice dynamics was realized as soon as appropriate computing resources were available. Early numerical computations on disordered systems were reviewed in 1972 by Dean [22] in a computational science article that is still interesting today. Much later, a pedagogical computational study of phonon propagation in disordered chains was performed by Allen and Kelner [23]. They showed that with increasing disorder, phonon propagation evolves from ballistic, to diffusive, to localization of the vibrational energy. The transitions between these three regimes are not abrupt. With modest disorder, some phonon wavepackets that propagate ballistically can be seen to scatter off the stronger inhomogeneities, and again propagate ballistically to their next scattering. These computational studies, although informative in their own right, were not directed towards the study of vibrational entropy.

\section{Bond Proportion Model}

If two phases differ in their energies by $\Delta E$ and entropies by $\Delta S$, these two phases will have the same free energy (i.e., $\Delta F=\Delta E-T_{\mathrm{c}} \Delta S=0$ ) at the critical temperature $T_{\mathrm{c}}$, determined by the condition $\Delta E=T_{\mathrm{c}} \Delta S$. Now suppose that $T_{\mathrm{c}}$ has been evaluated for the difference in configurational entropy alone. Calling it $T_{\mathrm{c}}^{\mathrm{conf}}$, we have $\Delta E=T_{\mathrm{c}}^{\mathrm{conf}} \Delta S_{\text {conf }}$. Now if, for the same $\Delta E$, we add a difference in vibrational entropy $\Delta S_{\text {vib }}$ to the model, the critical temperature of the phase transformation is changed to $T_{\mathrm{c}}^{\mathrm{conf}+\mathrm{vib}}$. In the classical limit, this new critical temperature is related to the critical temperature with configurational entropy alone as: $T_{\mathrm{c}}^{\mathrm{conf}+\mathrm{vib}}\left(\Delta S_{\text {conf }}+\Delta S_{\mathrm{vib}}\right)=T_{\mathrm{c}}^{\mathrm{conf}} \Delta S_{\text {conf }}$. There are handy methods for evaluating

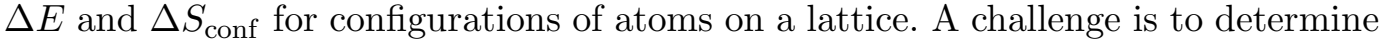
how $\Delta S_{\text {vib }}$ depends on the configurations of atoms, so that a new critical temperature can be obtained for a more complete model of a phase transformation of atoms on a lattice.

The vibrational entropy of phase transformations depends on changes in bond stiffnesses (force constants), so a natural first approach to estimate the vibrational entropy of a phase transformation is to count the different types of bonds between neighboring atoms, and how they change after a phase transformation. With a little risk, the accounting can be confined to first-nearest-neighbors (1NN), and with more risk we can associate the stronger bonds with the stiffer springs. This reasoning is enough to explain qualitatively the effects of vibrational entropy on the critical temperature, where a disordered phase at high temperatures transforms upon cooling to either an unmixed or ordered structure. The disordered phase has more bonds that are energetically unfavorable, and therefore weaker springs between atoms. A 
disordered phase is expected to have the larger vibrational entropy, increasing its relative stability. The critical temperature for disordering is therefore expected to decrease when vibrational entropy is added to the configurational entropy, and this is the usual case for real alloys. Assigning a fixed stiffness to a particular type of chemical bond, and counting these bonds, is called the "bond proportion model." It has a long history $[9,24,25,26,27,28]$.

Unfortunately, for quantitative predictions of the vibrational entropies of alloy phases, the bond proportion model is rarely adequate. Local atomic configurations alter the bond stiffnesses of atom pairs, and Section 5 discusses such effects parameterized by bond lengths and materials chemistry. Chemical effects are also discussed in Sections 18-20, including effects of temperature.

\subsection{Bond Proportion Model and Unmixing on the Ising Lattice}

Consider a binary alloy with atom species A and B arranged randomly on the sites of a crystal. The concentration of B-atoms is $c$, and the concentration of Aatoms is $1-c$. The crystal has $N$ sites, and therefore a number $c N$ of B-atoms and $(1-c) N$ of A-atoms. Each site is surrounded by $z$ sites as first-nearest-neighbors $(1 \mathrm{NN})$. For simplicity, we make the "point approximation," and assume that the chemical potential of an atom on a site depends on only the average occupancy of its neighboring sites. For a specified overall composition, we obtain thermodynamic functions with only knowledge of the species of atom on each site.

To obtain the total energy of an alloy configuration, we count the number of $1 \mathrm{NN}$ pairs: $\mathrm{A}-\mathrm{A}, \mathrm{B}-\mathrm{B},(\mathrm{A}-\mathrm{B}=\mathrm{B}-\mathrm{A})$, each with energy $e_{\mathrm{AA}}, e_{\mathrm{BB}}, e_{\mathrm{AB}}$. For a random solid solution, on average each $\mathrm{A}$-atom is surrounded by $z c \mathrm{~B}$-atoms, and its other $(1-c) z$ neighbors are A-atoms. Attributing all of the energy of the average A-atom, $e_{\mathrm{A}}$, to its $1 \mathrm{NN}$ chemical environment

$$
\begin{aligned}
e_{\mathrm{A}} & =\frac{z}{2}(1-c) e_{\mathrm{AA}}+\frac{z}{2} c e_{\mathrm{AB}} \\
e_{\mathrm{B}} & =\frac{z}{2}(1-c) e_{\mathrm{AB}}+\frac{z}{2} c e_{\mathrm{BB}}
\end{aligned}
$$

(and likewise for the average B-atom energy, $e_{\mathrm{B}}$ ). The division by 2 corrects for the double-counting that occurs when performing the average by summing over all atoms in the crystal (because the bonds from the neighbor atoms back to our central atom are counted again). The energies $e_{\mathrm{A}}$ and $e_{\mathrm{B}}$ also include the vibrational energy of the A- and B-atoms, which is $3 k_{\mathrm{B}} T$ in the classical limit. Since this is the same for both types of atoms, however, it does not affect the present analysis of phase transformations on an Ising lattice.

We define the "interchange energy"

$$
V \equiv\left(e_{\mathrm{AA}}+e_{\mathrm{BB}}-2 e_{\mathrm{AB}}\right) / 4 .
$$

The physical meaning of $V$ is made clear when we consider the interchange of an A$\mathrm{B}$ pair as shown in Fig. 2. For the initial configuration of the central A and B-atoms in Fig. 2a, we count $5 \mathrm{~A}-\mathrm{B}$ pairs, $1 \mathrm{~A}-\mathrm{A}$ pair, and $1 \mathrm{~B}-\mathrm{B}$ pair. After interchange of only the central A and B-atoms, ${ }^{7}$ we have 3 A-B pairs, 2 A-A pairs, and 2 B-B pairs

\footnotetext{
7 Note that this interchange conserves the total number of atoms of each type.
} 
Vibrational Thermodynamics of Materials
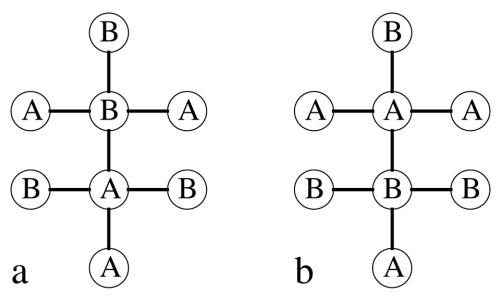

Figure 2. (a) Initial configuration around central A and B-atoms. (b) New configuration after exchange of the central A-B pair.

(Fig. 2b). The change in energy is precisely $4 V=e_{\mathrm{AA}}+e_{\mathrm{BB}}-2 e_{\mathrm{AB}}$ (or an integer multiple of $4 V$ for different configurations).

The partition function for a single site, $Z_{1 \text { site }}$ is

$$
Z_{1 \text { site }}=\mathrm{e}^{-\beta e_{\mathrm{A}}}+\mathrm{e}^{-\beta e_{\mathrm{B}}},
$$

where $\beta \equiv 1 /\left(k_{\mathrm{B}} T\right)$. Assuming all site occupancies are independent, the total partition function of the alloy with $N$ sites is

$$
Z_{N}=Z_{1 \text { site }}^{N}
$$

We can evaluate $Z_{N}$ by the binomial expansion of Eq. 44 plus Eq. 13 for the high temperature limit. For a fixed $n=c N$, we select only one term of the binomial expansion:

$$
Z_{N}=\frac{N !}{(N-n) ! n !}\left(\mathrm{e}^{-\beta e_{\mathrm{A}}}\right)^{N-n}\left(\mathrm{e}^{-\beta e_{\mathrm{B}}}\right)^{n} \prod_{j=1}^{m}\left(\frac{k_{\mathrm{B}} T}{\hbar \omega_{j}}\right)^{3 N g\left(\hbar \omega_{j}\right) \hbar \Delta \omega} .
$$

Evaluating the last factor in Eq. 45, the partition function for the vibrational frequencies, requires an approximation. We expect a physical correspondence between nearest-neighbor pairs and interatomic force constants. Section 2.4 showed how the normal mode frequencies $\left\{\omega_{j}\right\}$ are obtained from coupled equations of motion, and in principle a full diagonalization of the dynamical matrix may be required for each composition. This is difficult in practice, since disordered solid solutions cannot be described as periodic structures with small unit cells. On the other hand, we note that in Eq. 45 we need products of all the frequencies, not necessarly the individual frequencies. Mahanty and Sachdev [29] and Bakker [30, 31] addressed this problem, and showed for some crystals how the product of the $\left\{\omega_{j}^{2}\right\}$ equals the product of all interatomic force constants, $\left\{\gamma_{i j}\right\}$ between atom pairs, $i-j$, divided by the product of all atom masses, which is constant in a closed system. This result is consistent with the following approach of adapting the Einstein model for vibrational dynamics to our thermodynamic point approximation.

In the Einstein model each atom vibrates independently, as if tethered to a lattice site. $^{8}$ Its motions in three dimensions are decoupled, so each atom mass has three independent springs. The correspondence works naturally for a linear chain, square

\footnotetext{
8 The mass of the oscillator is therefore the mass of the atom, not the reduced mass of the atom plus its neighbor. In this approximation, the Einstein model preserves the independence of mass discussed in Section 3.1.
} 
lattice, or simple cubic lattice, for which each atom is associated with 1,2 , or 3 interatomic springs, with a frequency $\omega_{i j}$ that depends on the 1NN pair of atoms, $i$ and $j$. For a simple cubic lattice with $z=6$, each atom has $z / 2$ neighbors (bonds are not overcounted), and $z / 2$ springs (normal modes are not overcounted). When the coordination number $z$ is not the same as $2 \mathcal{D}$, where $\mathcal{D}$ is the number of dimensions, we correct the mode counting with the ratio $\eta=2 \mathcal{D} / z$, which is $1 / 2$ for an fcc crystal, for example. The vibrational factors in Eq. 45 now include only the frequencies $\left\{\omega_{\mathrm{AA}}, \omega_{\mathrm{BB}}, \omega_{\mathrm{AB}}\right\}$. Their exponents for the numbers of modes are replaced by the numbers of bonds of types A-B, A-A, B-B, adjusted to conserve modes, i.e., $\eta N_{\mathrm{AB}}$, $\eta N_{\mathrm{AA}}, \eta N_{\mathrm{BB}}$, giving

$$
\begin{aligned}
Z_{N}(c) & \simeq\left(\frac{N !}{(N-n) ! n !}\right)\left(\mathrm{e}^{-\beta e_{\mathrm{A}}}\right)^{N-n}\left(\mathrm{e}^{-\beta e_{\mathrm{B}}}\right)^{n} \\
& \times\left(\frac{k_{\mathrm{B}} T}{\hbar \omega_{\mathrm{AB}}}\right)^{\eta N_{\mathrm{AB}}}\left(\frac{k_{\mathrm{B}} T}{\hbar \omega_{\mathrm{AA}}}\right)^{\eta N_{\mathrm{AA}}}\left(\frac{k_{\mathrm{B}} T}{\hbar \omega_{\mathrm{BB}}}\right)^{\eta N_{\mathrm{BB}}} .
\end{aligned}
$$

To obtain $N_{\mathrm{AA}}$ for a random solid solution, we consider the average number of A-atom neighbors of an A-atom to be $(1-c) z$, so:

$$
N_{\mathrm{AA}}=(1-c)^{2} \frac{z N}{2},
$$

where dividing by 2 avoids double-counting. Likewise, $N_{\mathrm{BB}}=c^{2} z N / 2$ and $N_{\mathrm{AB}}=$ $c(1-c) z N$ (where we add A-B and B-A bonds together).

Obtaining the Helmholtz free energy from the expression $F(c)=-k_{\mathrm{B}} T \ln Z(c)$, and using the Stirling approximation $\ln (x !) \simeq x \ln x-x$, we obtain from Eq. 46

$$
F(c)=E_{\mathrm{conf}}(c)-T\left[S_{\mathrm{conf}}(c)+S_{\mathrm{vib}}(c)\right]
$$

where:

$$
\begin{aligned}
E_{\mathrm{conf}}(c) & =\left[e_{A A}+2 c\left(e_{A B}-e_{A A}\right)+c^{2}(4 V)\right] \frac{z N}{2} \\
S_{\mathrm{conf}}(c) & =-k_{\mathrm{B}}[c \ln c+(1-c) \ln (1-c)] N \\
S_{\mathrm{vib}}(c) & =k_{\mathrm{B}}\left[\mathcal{L}_{\mathrm{AA}}+2 c\left(\mathcal{L}_{\mathrm{AB}}-\mathcal{L}_{\mathrm{AA}}\right)+c^{2}\left(\mathcal{L}_{\mathrm{AA}}+\mathcal{L}_{\mathrm{BB}}-2 \mathcal{L}_{\mathrm{AB}}\right)\right] \frac{z \eta N}{2}, \\
S_{\mathrm{vib}}(c) & =k_{\mathrm{B}}\left[\ln \frac{k_{\mathrm{B}} T}{\hbar \omega_{\mathrm{AA}}}+2 c \ln \frac{\omega_{\mathrm{AA}}}{\omega_{\mathrm{AB}}}+c^{2} \ln \frac{\omega_{\mathrm{AB}}^{2}}{\omega_{\mathrm{AA}} \omega_{\mathrm{BB}}}\right] \mathcal{D} N .
\end{aligned}
$$

Here $E_{\text {conf }}(c), S_{\text {conf }}(c)$ and $S_{\text {vib }}(c)$ are the configurational energy, configurational entropy, and vibrational entropy of the random alloy. In Eq. 51 factors were defined such as $\mathcal{L}_{\mathrm{AB}} \equiv \ln \left(k_{\mathrm{B}} T /\left(\hbar \omega_{\mathrm{AB}}\right)\right)$. In the classical limit of the Planck distribution, $n\left(\varepsilon_{\mathrm{AB}}, T\right)$ of Eq. 7 , this $\mathcal{L}_{\mathrm{AB}}$ is the logarithm of the number of phonons of frequency $\omega_{\mathrm{AB}}$. Additionally, $k_{\mathrm{B}} \mathcal{L}_{\mathrm{AB}}$ is the vibrational entropy of an oscillator of frequency $\omega_{\mathrm{AB}}$, Eq. 10 in the classical limit. Equations 49 and 51 show that $E_{\text {conf }}(c)$ and $S_{\text {vib }}(c)$ have identical quadratic forms in $c$, reflecting the energies or interatomic force constants of nearest-neighbor bonds in the point (Bragg-Williams) approximation.

For chemical unmixing we consider the case where $V<0$ (from Eq. 42 this means that the average of A-A and B-B pairs is more favorable, i.e., their energies 

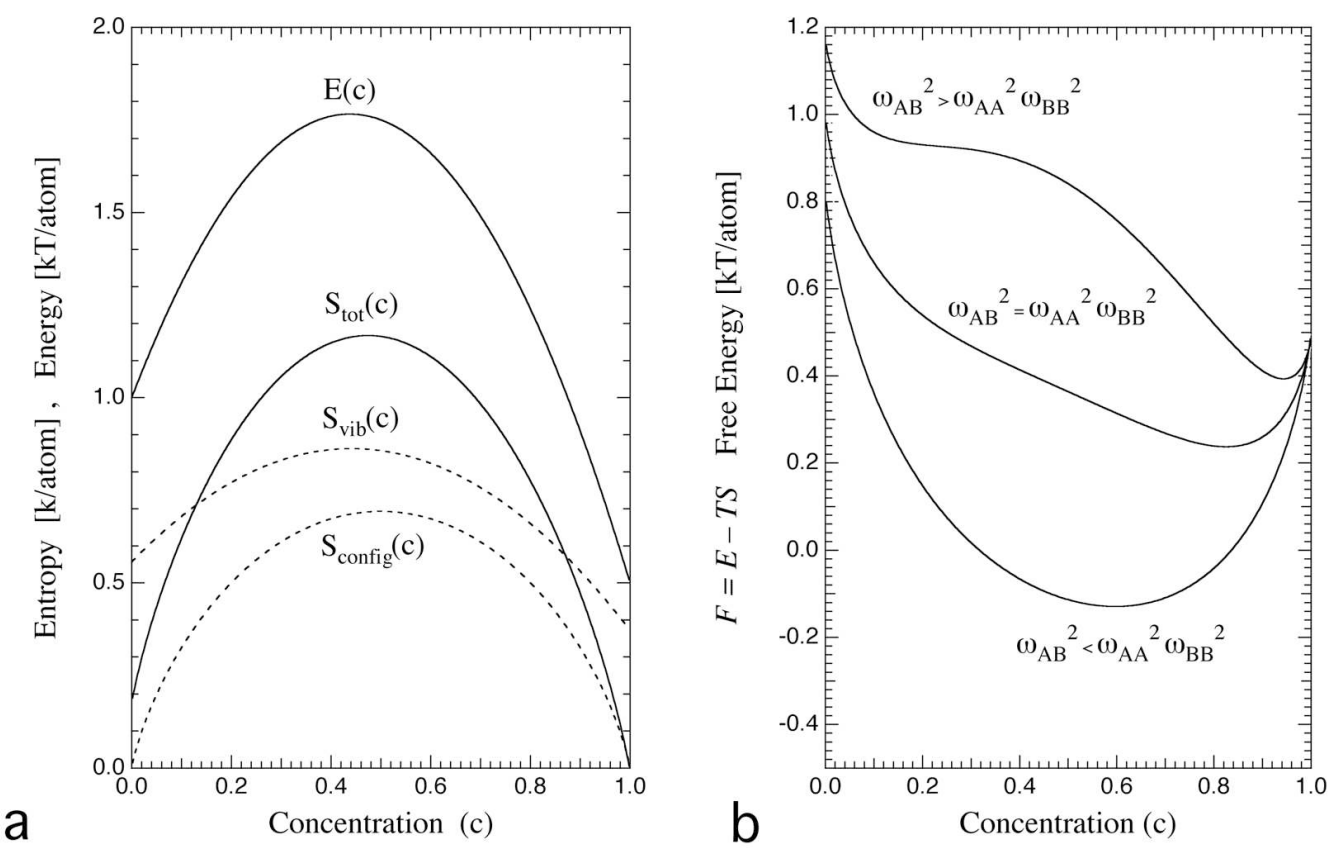

Figure 3. (a) $S_{\text {config }}(c)$ for random solid solution (Eq. 50), $E_{\text {config }}(c)$ for unmixing, i.e., $V<0$ (Eq. 49), representative $S_{\mathrm{vib}}(c)$ when $\omega_{\mathrm{AB}}^{2}<\omega_{\mathrm{AA}} \omega_{\mathrm{BB}}$ (Eq. 52), and $S_{\text {tot }}(c)=S_{\text {config }}(c)+S_{\mathrm{vib}}(c)$. (b) Free energy for $z V /(k T)=1$ (critical condition at $c=0.5$ without vibrational entropy), showing effects of different ratios of $\omega_{\mathrm{AB}}^{2}$ to $\omega_{\mathrm{AA}} \omega_{\mathrm{BB}}$.

are more negative, than A-B pairs). ${ }^{9}$ The energy and entropy terms of Eqs. 49, 50 and 52 are graphed in Fig. 3a. It was assumed that $\omega_{\mathrm{AB}}=0.77 \sqrt{\omega_{\mathrm{AA}} \omega_{\mathrm{BB}}}$, and $\omega_{\mathrm{AA}}<\omega_{\mathrm{BB}}$, as may be plausible if $e_{\mathrm{AA}}>e_{\mathrm{BB}}$. Figure $3 \mathrm{~b}$ shows curves of the total free energy versus composition for different ratios of vibrational frequencies. The logarithmic singularity of $S_{\text {config }}(c)$ always forces a downwards slope away from $c=0$ and $c=1$. The energy term, positive in this case with $V<0$, promotes unmixing at intermediate concentrations at low temperatures.

The critical temperature for chemical unmixing, $T_{\mathrm{c}}$, is found by setting $\partial^{2} F / \partial c^{2}=$ 0. It is straightforward to do this with Eqs. 49 through 52, with the result

$$
k_{\mathrm{B}} T_{\mathrm{c}}=\frac{4|V| z}{\frac{1}{c(1-c)}+2 \mathcal{D} \ln \left(\frac{\omega_{\mathrm{AA}} \omega_{\mathrm{BB}}}{\omega_{\mathrm{AB}}^{2}}\right)} .
$$

The $T_{\mathrm{C}}$ is reduced by vibrational entropy when $\omega_{\mathrm{AA}} \omega_{\mathrm{BB}}>\omega_{\mathrm{AB}}^{2}$, and increased when $\omega_{\mathrm{AA}} \omega_{\mathrm{BB}}<\omega_{\mathrm{AB}}^{2}$.

When $\omega_{\mathrm{AB}}^{2}=\omega_{\mathrm{AA}} \omega_{\mathrm{BB}}$, Eq. 53 provides $T_{\mathrm{c}}=z|V| / k_{\mathrm{B}}$ for $c=0.5$; the familiar result without vibrational entropy. The middle curve in Fig. 3b shows $F(c)$ for this condition. The free energy without vibrational entropy, $F_{\text {config }}(c)$, has an interesting curvature in $c$ below $T_{\mathrm{c}}=z|V| / k_{\mathrm{B}}$, which can be helpful for understanding spinodal decomposition.

\footnotetext{
9 The opposite sign of $V>0$ means that at low temperatures the alloy will tend to maximize the number of its A-B pairs by developing chemical order, as described in the next section.
} 
When $\omega_{\mathrm{AB}}^{2} \neq \omega_{\mathrm{AA}} \omega_{\mathrm{BB}}, S_{\mathrm{vib}}(c)$ is a quadratic function of $c$ (Eq. 52), much like $E_{\text {config }}(c){ }^{10}$

When $\omega_{\mathrm{AB}}^{2}<\omega_{\mathrm{AA}} \omega_{\mathrm{BB}}$, the vibrational entropy term $-T S_{\mathrm{vib}}(c)$ effectively diminishes $E_{\text {config }}(c)$, reducing $T_{\mathrm{c}}$.

When $\omega_{\mathrm{AB}}^{2}>\omega_{\mathrm{AA}} \omega_{\mathrm{BB}}, T_{\mathrm{c}}$ is raised and the whole unmixing phase boundary moves to higher temperatures. If $\omega_{\mathrm{AB}}^{2} \exp (-2 / 3)>\omega_{\mathrm{AA}} \omega_{\mathrm{BB}}$ (approximately $\left.0.513 \omega_{\mathrm{AB}}^{2}>\omega_{\mathrm{AA}} \omega_{\mathrm{BB}}\right)$, disordering cannot occur at any temperature at $c=0.5$. For yet larger $\omega_{\mathrm{AB}}^{2}$ with respect to $\omega_{\mathrm{AA}} \omega_{\mathrm{BB}}$, the entropy of mixing quickly becomes negative for intermediate compositions, suppressing disordering. A negative entropy of mixing has been observed in alloys of V-Pt at surprisingly low concentrations of Pt (see Sect. 20.3.2).

\subsection{Bond Proportion Model and Ordering on the Ising Lattice}

For chemical ordering, we consider the case $V>0$ of Eq. 42, where the energetics tends to maximize the number of A-B pairs at low temperatures. At high temperatures, we expect this alloy to become a random solid solution if the configurational entropy is dominant. For illustration of an ordering transformation we choose an equiatomic A-B alloy on the square lattice shown in Fig. 4. Figure 4 identifies the $\alpha$ - and $\beta$-sublattices, each having $N / 2$ sites for a crystal with $N$ sites. Finite temperatures favor some disorder in the structure - putting a few atoms on the wrong sublattice gives a big increase in the configurational entropy, ${ }^{11}$ driving some mixing of A-atoms onto the $\beta$-sublattice, in exchange for B-atoms on the $\alpha$-sublattice.

Figure 4 motivates the definition of four types of sublattice concentration variables: $\left\{N_{\mathrm{A}}^{\alpha}, N_{\mathrm{A}}^{\beta}, N_{\mathrm{B}}^{\alpha}, N_{\mathrm{B}}^{\beta}\right\}$, where for example $N_{\mathrm{A}}^{\alpha}$ is the number of A-atoms on the $\alpha$ sublattice. For an equiatomic alloy, by conservation of atoms and conservation of sublattice sites, $N_{\mathrm{A}}^{\alpha}=N_{\mathrm{B}}^{\beta} \equiv R$, where $R$ denotes atoms that are on the "right" sublattice, and $N_{\mathrm{A}}^{\beta}=N_{\mathrm{B}}^{\alpha} \equiv W$, denoting "wrong" atoms. The sublattice concentrations are used to define a "long-range order" (LRO) parameter, $L$, for the alloy $[32,33,34]$

$$
L \equiv \frac{R-W}{N / 2} .
$$

This $L$ can range from $-1<L<1$. The case of $L=1$ corresponds to perfect order where $R=N / 2$ and $W=0$. The disordered alloy has as many right as wrong atoms on each sublattice, i.e., $R=W$, so $L=0$ for a disordered alloy.

Analogous to the assumption of a random solid solution, we assume that the atoms are placed randomly on a sublattice, e.g., the $N_{\mathrm{A}}^{\alpha} \mathrm{A}$-atoms have no preference for any site on the $\alpha$-sublattice, irrespective of the presence of A-atoms or B-atoms on neighboring sites. This is the "point" or "Bragg-Williams" approximation. The strength of this approximation is that it makes it easy to count the bonds between the different species of atoms.

\footnotetext{
10 A simple approximation is that the strengths of the bonds and spring constants are proportional, giving $S_{\mathrm{vib}}(c)$ the same shape as $E_{\text {config }}(c)$. This is conceptually convenient, and drove some early studies, but it has weak predictive power.

11 The entropy has a logarithmic singularity in sublattice concentrations analogous to the concentration dependence of Eq. 50 near $c=0$.
} 


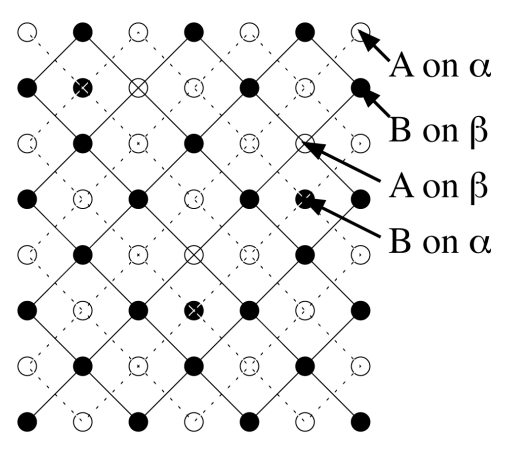

Figure 4. Ordered square lattice with two species $\{\mathrm{A}, \mathrm{B}\}$. Dashed and solid lines represent two interpenetrating square sublattices $\{\alpha, \beta\}$. A few misplaced $\mathrm{A}$ and $\mathrm{B}$ atoms are shown.

To determine the numbers of pairs $N_{\mathrm{AA}}, N_{\mathrm{BB}}, N_{\mathrm{AB}}$, an important point evident from Fig. 4 is that the first neighbors of each atom on the $\alpha$-sublattice are on the $\beta$-sublattice, and vice-versa. When evaluating $N_{\mathrm{AA}}$ we start with all $R$ A-atoms on the $\alpha$ sublattice, and multiply by the probability of finding a wrong A-atom in each of the $z$ neighboring sites of the $\beta$ sublattice (which has $N / 2$ sites).

$$
\begin{aligned}
N_{\mathrm{AA}}=N_{\mathrm{BB}} & =R W \frac{z}{N / 2}, \\
N_{\mathrm{AB}} & =\left(R^{2}+W^{2}\right) \frac{z}{N / 2} .
\end{aligned}
$$

Using Eqs. 54 through 56

$$
\begin{aligned}
N_{\mathrm{AA}}=N_{\mathrm{BB}} & =\left(1-L^{2}\right) \frac{z N}{8}, \\
N_{\mathrm{AB}} & =\left(1+L^{2}\right) \frac{z N}{4} .
\end{aligned}
$$

In the high temperature limit for phonons, we write the partition function for the alloy in terms of the random occupancies on two sublattices (giving two binomial probabilities) and three factors that depend on the numbers of bonds:

$$
\begin{aligned}
Z=\left(\frac{(N / 2) !}{R ! W !}\right)^{2} & {\left[\mathrm{e}^{-\beta e_{\mathrm{AA}}}\left(\frac{k_{\mathrm{B}} T}{\hbar \omega_{\mathrm{AA}}}\right)^{\eta}\right]^{N_{\mathrm{AA}}}\left[\mathrm{e}^{-\beta e_{\mathrm{BB}}}\left(\frac{k_{\mathrm{B}} T}{\hbar \omega_{\mathrm{BB}}}\right)^{\eta}\right]^{N_{\mathrm{BB}}} } \\
& \times\left[\mathrm{e}^{-\beta e_{\mathrm{AB}}}\left(\frac{k_{\mathrm{B}} T}{\hbar \omega_{\mathrm{AB}}}\right)^{\eta}\right]^{N_{\mathrm{AB}}} .
\end{aligned}
$$

As for Eq. 46, we seek a correspondence between the number of modes and the number of pairs. For the square lattice Eq. 59 works naturally with $\eta=1$, but for other lattices it is necessary to take care to avoid incorrect counting of the $3 \mathrm{~N}$ normal modes by setting $\eta=2 \mathcal{D} / z$, where $\mathcal{D}$ is the dimensionality.

The partition function depends on the LRO parameter, $L$, and we replace the exponents in Eq. 59 with Eqs. 57-58, and use Eq. 54 with the conservation of sites $(R+W=N / 2)$ to remove all instances of $R, W, N_{\mathrm{AA}}, N_{\mathrm{BB}}, N_{\mathrm{AB}}$ in Eq. 59, obtaining $Z(L)$. The free energy is obtained as $F(L)=-k_{\mathrm{B}} T \ln Z(L)$. Subtracting the free energy of the disordered solid solution, we obtain the free energy of ordering, 


$$
\begin{aligned}
\Delta F(L) \equiv F(L)-F(L & =0) \\
\Delta F(L)= & -L^{2} \frac{z N}{8} 4 V \\
& -T \frac{N}{2} k_{\mathrm{B}}[-(1+L) \ln (1+L)-(1-L) \ln (1-L)] \\
& -T L^{2} \frac{\mathcal{D} N}{4} k_{\mathrm{B}} \ln \left(\frac{\omega_{\mathrm{AA}} \omega_{\mathrm{BB}}}{\omega_{\mathrm{AB}}^{2}}\right)
\end{aligned}
$$

where the three lines of Eq. 60 are identified as the changes upon ordering of $E_{\text {config, }},-T S_{\text {config }}$, and $-T S_{\text {vib }}$, respectively. We set $\partial \Delta F(L) / \partial L=0$ and obtain a transcendental equation for the equilibrium value of $L(T)$

$$
2 L z V=k_{\mathrm{B}} T \ln \left(\frac{1+L}{1-L}\right)+L \mathcal{D} k_{\mathrm{B}} T \ln \left(\frac{\omega_{\mathrm{AB}}^{2}}{\omega_{\mathrm{AA}} \omega_{\mathrm{BB}}}\right) .
$$

This differs from the usual Bragg-Williams solution by the extra term from vibrational entropy. This term is linear in $L$, so it can be considered as a temperaturedependent rescaling of the interatomic exchange energy, $V$.

There is no analytical solution to Eq. 61 for all $T$, but it is possible to obtain an analytic solution for the critical temperature, $T_{\mathrm{c}}$. The trick is to assume $L$ is infinitesimally small, so Eq. 61 can be linearized. ${ }^{12}$ The result is

$$
k_{\mathrm{B}} T_{\mathrm{c}}=\frac{z V}{1+\frac{\mathcal{D}}{2} \ln \left(\frac{\omega_{\mathrm{AB}}^{2}}{\omega_{\mathrm{AA}} \omega_{\mathrm{BB}}}\right)} .
$$

In the absence of vibrational entropy, i.e., $\omega_{\mathrm{AB}}^{2}=\omega_{\mathrm{AA}} \omega_{\mathrm{BB}}$, Eq. 62 reduces to $T_{\mathrm{c}}=z V / k_{\mathrm{B}}$, a well-known result of Bragg-Williams theory. The configurational entropy is always important in a chemical order-disorder transformation. When both vibrational and configurational entropies are important, if $\omega_{\mathrm{AB}}^{2}>\omega_{\mathrm{AA}} \omega_{\mathrm{BB}}$ the Bragg-Williams value of $T_{\mathrm{C}}$ is reduced, perhaps significantly. In the case where $\omega_{\mathrm{AB}}^{2}=1.1 \omega_{\mathrm{AA}} \omega_{\mathrm{BB}}$, for a simple cubic lattice $T_{\mathrm{c}}$ is reduced to $87 \%$ of its value without vibrational entropy. This suppression of $T_{\mathrm{c}}$ seems to be the usual effect of vibrational entropy, but the opposite is possible when $\omega_{\mathrm{AB}}^{2}<\omega_{\mathrm{AA}} \omega_{\mathrm{BB}}$. Although difficult to expect from physical intuition, in the case where $\omega_{\mathrm{AB}}^{2} / \omega_{\mathrm{AA}} \omega_{\mathrm{BB}}<$ $\exp (-2 / 3)$, vibrational entropy suppresses disordering, and $T_{\mathrm{c}}=\infty$.

\subsection{Monte Carlo Results}

Some of the earliest computational studies on the vibrational entropy of ordering were performed by H. Bakker [30, 31]. Building on work by Mahanty and Sachdev [29], Bakker used the result for the difference in vibrational entropy of phases $\alpha$ and

\footnotetext{
12 This assumes that the ordering transformation remains second order, which is true for Eq. 61. The curve of $L(T)$ from Bragg-Williams theory, which decreases continuously from $L=1$ to $L=0$ at $T_{\mathrm{c}}$, is rescaled monotonically with $S_{\mathrm{vib}}$ - the effective $V$ can be considered as altered by a temperature-dependent term.
} 


$$
\Delta S_{\mathrm{vibr}}=1 / 2 \ln \frac{\left|\underline{B}^{\alpha}\right|\left|\underline{M}^{\beta}\right|}{\left|\underline{M}^{\alpha}\right|\left|\underline{B}^{\beta}\right|}
$$

where the determinant of the mass matrix $\underline{M}$ is the product of the atomic masses, and undergoes no change with ordering. The force constant matrix is $\underline{B}$. In the case of a simple cubic A-B alloy with first-neighbor interatomic forces $\gamma_{\mathrm{AA}}, \gamma_{\mathrm{BB}}, \gamma_{\mathrm{AB}}$, the resulting critical temperature is

$$
k_{\mathrm{B}} T_{\mathrm{c}}=\frac{z V}{1+\frac{z}{8} \ln \left(\frac{\gamma_{\mathrm{AB}}^{2}}{\gamma_{\mathrm{AA}} \gamma_{\mathrm{BB}}}\right)},
$$

in agreement with Eq. 62 because $\gamma \sim \omega^{2} M$.

Tuijn and Bakker $[35,36]$ computed the effects of vibrational entropy on the heat capacity of a three-dimensional crystal by counting the number of A-B pairs in a Monte Carlo simulation. Tuijn's results are shown in Fig. 5 for various values of $\Delta \equiv \gamma_{\mathrm{AB}} /\left[\gamma_{\mathrm{AA}} \gamma_{\mathrm{BB}}\right]^{-1 / 2}$. As expected, with $\Delta>1$, and hence stiffer vibrations in the ordered phase, the critical temperature is reduced. The critical temperature is reduced by approximately a factor of 1.8 when $\Delta=2$. The transformation still appears to be continuous, i.e., second order, although the singularity is rounded by the finite size of the lattice used in the simulations. Figure 5 shows that the more accurate Monte Carlo simulations predict a lower $T_{\mathrm{c}}$ than Eq. 64, a well-known result without vibrational entropy. The relative sensitivity to $\Delta$ is also different for the Bragg-Williams and Monte Carlo results, however. The best agreement between a rescaled Eq. 64 and Fig. 5 is found for $z=9$, rather than 6. Tuijn and Bakker also explored the effects of second nearest-neighbor interatomic forces and non-central forces. The heat capacities were similar to those of the simple cubic lattice with first-neighbor interactions, except when $\Delta \gg 2$.

\section{Bond-Stiffness-versus-Bond-Length Model}

\subsection{Phonon Frequencies And Bond Lengths}

The bond proportion model just described, which counts the different types of atom pairs, has a deficiency that is easy to understand. The bond lengths between pairs of atoms are different in different structures, and the stiffnesses of a bond is sensitive to the length of the bond. ${ }^{13}$ Counting the proportions of atom pairs is important when comparing the vibrational entropies of two different structures, but it is also important to know how each bond stiffness changes with bond length.

Consider a disordered solid solution with a distribution of interatomic distances, shorter and longer than optimal, owing to the different atomic sizes. The calculation of these distances is not simple because the relaxations in position for each atom depend in turn on the relaxations of its neighbors. Nevertheless, we can estimate

\footnotetext{
13 This same characteristic of the interatomic potential also gives rise to quasiharmonic theory, where interatomic forces weaken with thermal expansion.
} 


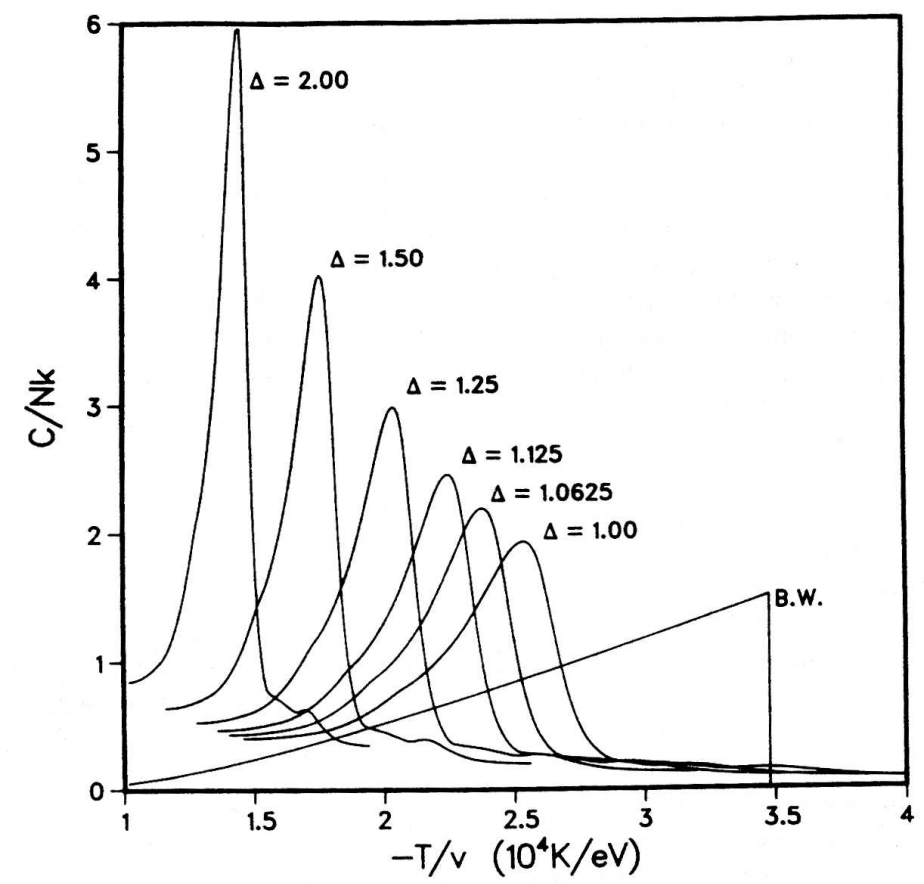

Figure 5. Heat capacity at constant volume for harmonic solids with different ratios of $\Delta$, as a function of $-T / v \equiv T / 2 \mathrm{~V}$ in the present notation. B.W. denotes Bragg-Williams approximation. After [37].

the size of this effect on phonon frequencies. The Grüneisen parameter $\gamma$ is defined as:

$$
\gamma \equiv-\frac{V}{\omega} \frac{\partial \omega}{\partial V}
$$

where $\omega$ is the phonon frequency and $V$ is volume. Recognizing that $V \propto r^{3}$ and $\omega \propto \sqrt{k_{s}}$, where $k_{s}$ is an interatomic force constant

$$
6 \gamma=-\frac{r}{k_{s}} \frac{\partial k_{s}}{\partial r}
$$

There is no universal value for a Grüneisen parameter, but typically $\gamma \simeq 2$. In this case, Eq. 66 predicts that if the interatomic distance changes by $+1 \%$, the interatomic force changes by $-12 \%$. This is a major effect. A change in interatomic distance of $+10 \%$ could cause the interatomic force to be lost entirely.

Grimvall and co-workers [38] correlated the interatomic forces of 8 alkali halides with the $\mathrm{NaCl}$ structure to their volume per atom in the crystal structure. (The interatomic forces were deduced from the bulk moduli.) The interatomic forces decreased approximately inversely with atomic volume per atom as $k_{s}=1300 / \mathrm{V}$ where the force (spring) constant $k_{s}$ has units of $\mathrm{N} / \mathrm{m}$ and $V$ has units of $10^{-30} \mathrm{~m}^{3}$. Fernández Guillermet and Grimvall sought similar trends in vanadium and titanium compounds with oxygen, nitrogen, and carbon [39]. The trend in this case was a rapid increase in the $k_{s}$ with $V$, and there was a significantly different slope for the 
titanium and vanadium compounds. Evidently the differences between these transition metal compounds are too large for a systematic relationship between $k_{S}$ and $V$, even though these also had the $\mathrm{NaCl}$ structure. Grimvall and Fernández-Guillermet correlated the thermodynamic Debye temperature to the averaged mass and volume of a series of $s p^{3}$-bonded compounds with the sphalerite $(\mathrm{ZnS})$ structure [40]. The correlation included InSb, GaP, GaAs, and the elements $\mathrm{Si}, \mathrm{Ge}$, and Sn were added to the plot. They found a relationship of the form $\Theta_{\mathrm{D}}[\mathrm{K}]=1500 a^{-1} M^{-1 / 2}\left[\mathrm{~nm}^{-1}\right.$ (at.wt.) $\left.{ }^{-1 / 2}\right]$ (where, for example $\Theta_{\mathrm{D}}=600 \mathrm{~K}$ corresponds to $a^{-1} M^{-1 / 2}=0.4$ in these units). Adding volume differences to these types of correlations for different compounds having the same structure accommodates somewhat their differences in bonding and bond stiffness. Suggestions that there should be a correlation between interatomic forces and atomic volume, assuming sufficient similarity in chemistry and structure of the compounds, have been around for many years [41].

Quantitative analyses of the effects of bond length on bond stiffnesses were performed by Sluiter, et al. on Al-Li [42] and by van de Walle and Ceder on Pd-V [43]. They tested the idea of transferring interatomic forces from one material to another, with the goal of predictive power without major calculations or measurements on every material. There is some subtlety to the concept of transferrability of force constants because different crystal structures have different sets of allowed tensorial force constants. Force constants must be identically zero if symmetry operations of the crystal transform them into their negatives. Different symmetry operations for different crystal structures therefore make it difficult to transfer tensorial force constants from one structure to another. This is not a problem for axial force models, which involve a central-force "stretching" force and a "bending" force, and axial force models often give adequate descriptions of lattice dynamics. In the "bondstiffness-versus-bond-length" approach, only first-nearest-neighbor $(1 \mathrm{NN})$ bonds are transferred between different structures $[9,44]$. It is also observed that only the stretching (longitudinal) forces between atoms have a significant dependence on the bond length. The bond bending forces are found to be smaller and less dependent on bond length.

Van de Walle and Ceder performed density-functional theory calculations of bond stiffnesses for a set of fcc-based Pd-V structures with different interatomic distances. Some results, presented in Fig. 6, show the longitudinal (stretching) interatomic forces between $\mathrm{V}-\mathrm{V}, \mathrm{Pd}-\mathrm{Pd}$, and $\mathrm{Pd}-\mathrm{V} 1 \mathrm{NN}$ pairs of atoms. The points are from full calculations for individual $\mathrm{Pd}-\mathrm{V}$ ordered compounds of different compositions and different lattice parameters. The points tend to fall rapidly with bond length, as expected when the interatomic forces soften with expansion. Approximately, there is a range of about $10 \%$ in the interatomic separation where the force constants vary from their maximum value to zero. Also approximately, the stretching stiffness has a linear relationship with bond length. The effects of bond length on bond stiffness of Fig. 6 are large, so a Pd-Pd bond cannot be expected to have the same stiffness in any local environment of a random a solid solution, for example. The bond-stiffness-versus-bond-length model is useful for semi-quantitative arguments, and when calibrated for specific elements by ab-initio calculations, for example, this model can be used for comparing phonon entropies of different alloy phases. The 


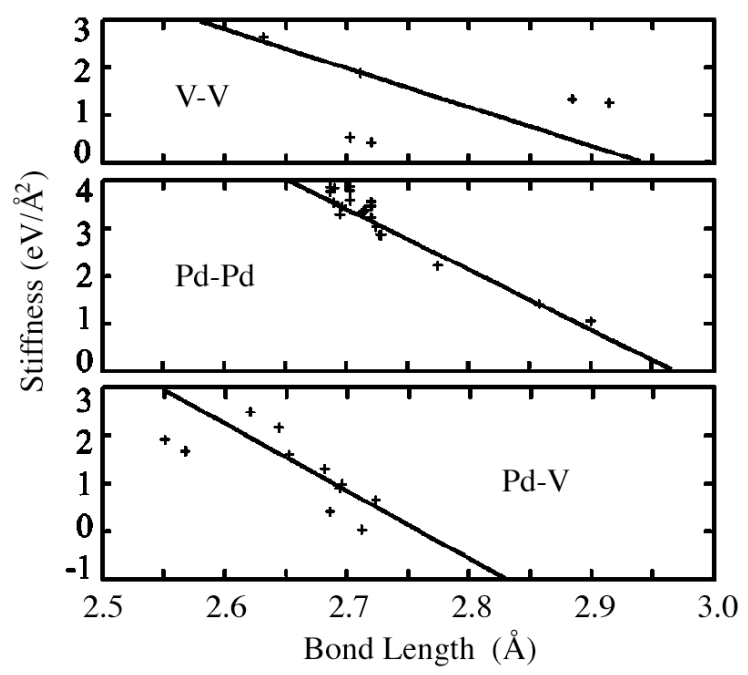

Figure 6. Stretching terms of the nearest-neighbor force constants as a function of bond length of $\mathrm{Pd}-\mathrm{V}$ alloys. Each point corresponds to one type of bond in one of a set of fcc crystal structures of different volumes. After [43].

bond-stiffness-versus-bond-length model would reduce to the bond proportion model if there were no variation of bond stiffness with interatomic distance.

\subsection{Extending the Bond Proportion Model}

Consider how a bond-stiffness-versus-bond-length relationship alters the free energy (Eq. 60) and the critical temperature (Eq. 62) of the bond proportion model for ordering transformations. The free energy $F(L)$ of Eq. 60 is with reference to the disordered solid solution, and is zero for $L=0$ (i.e., $F(0)=0$ ). Consider again the case of $\mathrm{B} 1$ ordering ( $\mathrm{NaCl}$ prototype) of an $\mathrm{AB}$ alloy on a simple cubic lattice, as discussed following Eq. 62. In this structure, all 1NN bonds are between A-B pairs, and there is only one bond length. It is the disordered solid solution where the distortions of bond lengths occur. This is a complex problem owing to the different relaxations in the different local environments. We can get to the essence of the problem by using the same Grüneisen parameter for all bonds (Eq. 65), and by simplifying the three curves of Fig. 6 by assuming that the bond stiffnesses are zero at the same bond lengths for A-A, B-B, and A-B pairs. In this case, all three frequencies $\left\{\omega_{\mathrm{AA}}, \omega_{\mathrm{BB}}, \omega_{\mathrm{AB}}\right\}$ change with volume as:

$$
\omega_{i j}=\omega_{i j}^{0}\left(1-\gamma \frac{\delta V}{V^{0}}\right)
$$

where the superscript 0 denotes a reference value for the ordered structure. For small $\delta V / V^{0}$

$$
\ln \left(\omega_{i j}\right) \simeq \ln \left(\omega_{i j}^{0}\right)-\gamma \frac{\delta V}{V^{0}} .
$$

To the free energy of Eq. 60 we add the term

$$
-T S_{\text {Gruen }}=k_{\mathrm{B}} T\left(1-L^{2}\right) \mathcal{D} N \gamma \frac{\delta V}{V^{0}},
$$


which vanishes when $L=1$, but grows with disorder as the number of R-W pairs increases as $1-L^{2}$. The critical temperature of Eq. 62 becomes:

$$
k_{\mathrm{B}} T_{\mathrm{c}}=\frac{z V}{1+2 \mathcal{D} \gamma \frac{\delta V}{V^{0}}+\frac{\mathcal{D}}{2} \ln \left(\frac{\omega_{\mathrm{AB}}^{2}}{\omega_{\mathrm{AA}} \omega_{\mathrm{BB}}}\right)} .
$$

Consider a typical case where the disordered phase has a lattice parameter $1 \%$ larger than the ordered phase (so $\delta V / V^{0} \simeq 0.03$ ). Assuming $\gamma=2$, the second term in the denominator of Eq. 70 is +0.36 . The critical temperature is suppressed substantially when the average bond length in the disordered solid solution is $1 \%$ longer than for the ordered phase. ${ }^{14}$ Because we assumed the same Grüneisen parameter for all bonds, this effect of bond stiffness versus bond length is independent of the effect on $T_{\mathrm{c}}$ from stiffness differences of the different pairs of atoms. In a more complete treatment these effects are inter-related.

\subsection{Chemical Effects on Bond Stiffness}

A closer examination of Fig. 6 shows that the bond stiffnesses for $\mathrm{V}-\mathrm{V}$ and $\mathrm{Pd}-\mathrm{V}$ pairs have considerable scatter about the linear fit. Some of this can be accounted for by a dependence of bond stiffness on chemical composition (discussed further in Sect. 20.1). For example, the addition of B atoms to a host crystal of element A will fill or deplete the electronic band structure of A, causing changes in the density of electron states at the Fermi level. The electron screening of the A-ion cores is thereby altered, changing the bond stiffnesses of A-A pairs. Such effects may scale with the electronegativity difference between $\mathrm{A}$ and $\mathrm{B}$ atoms, and features of the electronic structure such as the density of states at the Fermi level. These chemical effects are probably less significant for alloys of isoelectronic elements.

Liu, et al., studied the stretching forces in fcc aluminum alloyed with early transition metals [45], and presented a number of graphs such as Fig. 7. The stiffness of $\mathrm{Al}-\mathrm{Al}$ bonds decreases with $\mathrm{Zr}$ concentration. This effect is big, and is comparable to the change of bond stiffness versus bond length at one alloy composition. The Pauling electronegativity of $\mathrm{Al}$ (1.61) exceeds that of $\mathrm{Zr}$ (1.33), so alloying should cause substantial changes in electronic structure. Liu, et al., argue that when $\mathrm{Al}$ is alloyed with $\mathrm{Zr}$, electrons are transferred into the stronger Al-Zr bonds, stiffening them at the expense of $\mathrm{Al}-\mathrm{Al}$ bonds. The stretching forces between $\mathrm{Al}-\mathrm{Zr}$ and $\mathrm{Zr}-\mathrm{Zr}$ pairs are much less affected, however. These effects of alloying on bond stiffness in Al-Zr are discussed further in Section 11.

\section{Heat Capacity}

\subsection{Harmonic Heat Capacity}

First consider a harmonic model where $C_{V}$ is obtained with a fixed set of oscillator frequencies, or a fixed phonon DOS. The temperature derivative of the Planck

\footnotetext{
14 In the less common case where the lattice parameter of the disordered alloy is smaller than the ordered, $T_{\mathrm{c}}$ would be raised.
} 


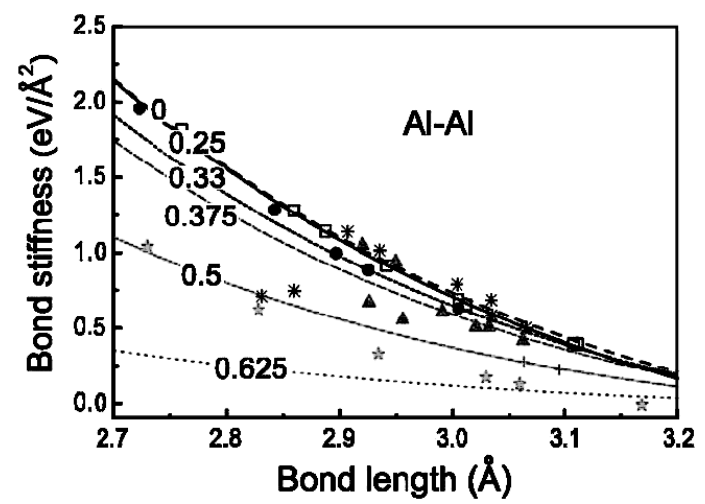

Figure 7. Nearest-neighbor stretching forces between Al-Al pairs in fcc Al-Zr alloys with Zr concentrations as labeled. After [45].

occupancy distribution (Eq. 7), weighted by the energy of the mode, $\varepsilon_{i}$, shows that each mode of energy $\varepsilon_{i}$ contributes to the heat capacity at $T$ the amount

$$
C_{V, i}(T)=k_{\mathrm{B}}\left(\frac{\varepsilon_{i}}{k_{\mathrm{B}} T}\right)^{2} \frac{\exp \left(\varepsilon_{i} / k_{\mathrm{B}} T\right)}{\left(\exp \left(\varepsilon_{i} / k_{\mathrm{B}} T\right)-1\right)^{2}} .
$$

In the high temperature (classical) limit where $k_{\mathrm{B}} T \gg \varepsilon_{i}$, the heat capacity becomes $1 k_{\mathrm{B}}$ per mode. ${ }^{15}$ Equation 71 is consistent with Eq. 6 if we integrate over $T$ for all modes

$$
S_{V, \mathrm{vib}}(T)=\int_{0}^{T} \sum_{i}^{3 N} \frac{C_{V, i}\left(T^{\prime}\right)}{T^{\prime}} \mathrm{d} T^{\prime} .
$$

(Usually we integrate against the phonon DOS, $g(\varepsilon)$, to obtain $S_{V, \text { vib }}(T)$.)

A Debye model predicts some general features of the heat capacity. Figure 8a shows heat capacity curves for two materials with similar Debye temperatures. The curves look similar at low $T$, and are essentially identical at very high $T$. Their most pronounced difference is found at approximately one-quarter of the Debye temperature. The integrand of Eq. 72 has a maximum below about a fifth of the Debye temperature, so experimental studies of vibrational entropy by heat capacity should include this range of temperatures. The difference between these two curves, shown in Fig. 8b, is typical of the shape of a differential heat capacity curve obtained from real phonon DOSs (see curve "DOS $11 \mathrm{~K}$ " in Fig. 26), although there are differences in detail. The Debye model assumes a phonon DOS that is a quadratic function of $\varepsilon$, and increases monotonically up to a cutoff energy $\varepsilon_{\mathrm{D}}$. The actual DOS often has a more abrupt rise at low energies that comes from the lower sound velocity of the low transverse modes, which are also weighted at their cutoff energy

\footnotetext{
15 Even with phonon softening this could remain true. In the high-temperature limit, with phonon occupancies of $k_{\mathrm{B}} T / \varepsilon_{i}$ per mode, the softening of the modes gives an increase in occupancy proportional to $1 / \varepsilon_{i}$, but a decrease in energy proportional to $\varepsilon_{i}$. The product of these two factors produces a heat capacity $C_{V}$ at high temperatures that is unaffected by mode softening, so $C_{V}$ can remain as $3 k_{\mathrm{B}}$ /atom at high temperatures. This is not so if there are other phenomena at work, such as a buildup of elastic energy as a crystal expands.
} 

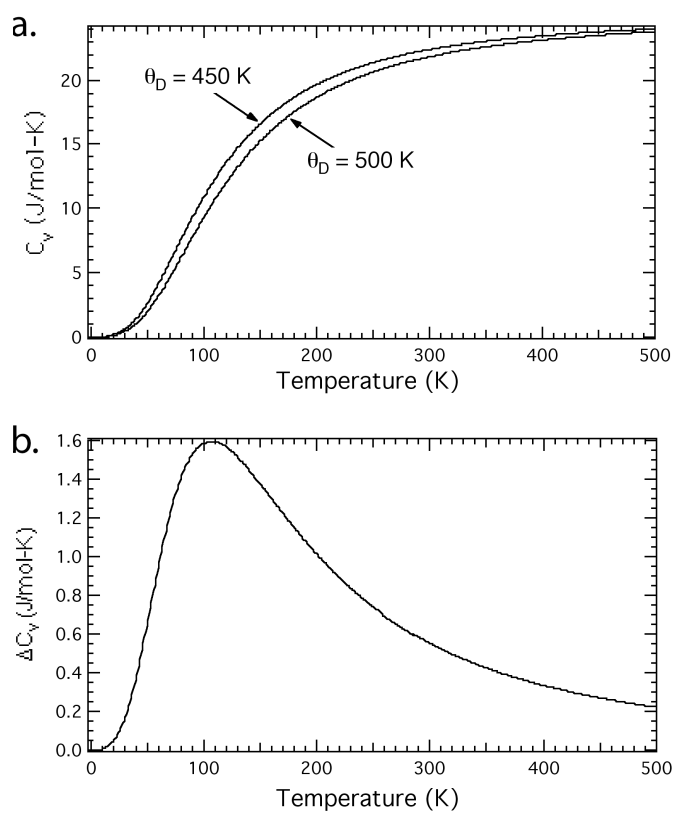

Figure 8. (a) Heat capacity versus temperature for two Debye solids with Debye temperatures of 450 and $500 \mathrm{~K}$. (b) Difference of the two curves in part a.

by the effects of dispersion (e.g., from a high DOS at the Brillouin zone boundary). Plots of $\varepsilon_{\mathrm{D}}$ versus $T$ typically show a dip and a rise from a mimimum at roughly a tenth of the Debye temperature $\theta_{\mathrm{D}}=\varepsilon_{\mathrm{D}} / k_{\mathrm{B}}$, but this varies from material to material.

\subsection{Quasiharmonic Thermodynamics}

More typically measured by calorimetry is the amount of heat going into the solid at constant pressure, $C_{p}$, defined as

$$
\left.C_{p}(T)=T \frac{\mathrm{d} S}{\mathrm{~d} T}\right)_{p} .
$$

The difference between $C_{p}$ and $C_{V}$ is a classical thermodynamic relationship

$$
C_{p}-C_{V}=9 B v \alpha^{2} T,
$$

where $B$ is bulk modulus, $\alpha$ is the linear coefficient of thermal expansion, $v$ is specific volume, and 9 is the square of three dimensions. Equation 74 can be derived from formal thermodynamic manipulations, aided for example by techniques of Jacobian transformations.

A physical derivation of Eq. 74 can be performed by considering the free energy of a crystal having only 1) phonons, and 2) thermal expansion. There are energy terms from the phonons and from the elastic expansion, and an entropy from the phonons (an average thermal expansion does not generate entropy itself). The free energy is

$$
F(T)=E_{\text {elas }}(T)+E_{\mathrm{ph}}(T)-T S_{\mathrm{ph}} .
$$


The elastic energy of thermal expansion, using $\delta V / V_{0}=3 \alpha T$ is

$$
\begin{aligned}
& E_{\text {elas }}=\frac{1}{2} B \frac{(\delta V)^{2}}{V_{0}}, \\
& E_{\text {elas }}=\frac{9}{2} B V_{0} \alpha^{2} T^{2},
\end{aligned}
$$

The phonon energy in the classical limit is

$$
E_{\mathrm{ph}}(T)=3 N k_{\mathrm{B}} T
$$

In the high-temperature limit, the phonon entropy is (cf., Eq. 15)

$$
S_{\mathrm{ph}}(T)=k_{\mathrm{B}} \sum_{j}^{3 N} \ln \left(\frac{k_{\mathrm{B}} T}{\hbar \omega_{j}}\right) .
$$

Using the same approach as Eq. 68 with $\omega_{j}=\omega_{j}^{0}\left(1-\gamma \delta V / V_{0}\right)$ and $\delta V / V_{0}=3 \alpha T$ for the thermal expansion

$$
\begin{aligned}
S_{\mathrm{ph}}(T) & =k_{\mathrm{B}} \sum_{j}^{3 N} \ln \left(\frac{k_{\mathrm{B}} T}{\hbar \omega_{j}^{0}(1-3 \gamma \alpha T)}\right), \\
S_{\mathrm{ph}}(T) & =\left[k_{\mathrm{B}} \sum_{j}^{3 N} \ln \left(\frac{k_{\mathrm{B}} T}{\hbar \omega_{j}^{0}}\right)\right]+9 N \gamma \alpha k_{\mathrm{B}} T, \\
S_{\mathrm{ph}}(T) & =S_{\mathrm{ph}}^{0}(T)+9 N \gamma \alpha k_{\mathrm{B}} T=S_{\mathrm{ph}}^{0}+S_{\mathrm{q}},
\end{aligned}
$$

where the first term, $S_{\mathrm{ph}}^{0}(T)$, is the harmonic phonon entropy with phonon frequencies characteristic of $T=0$, and unchanged with temperature. Using Eqs. 76, 77 and 81 in the free energy expression for the expanded crystal with phonons, Eq. 75

$$
F(T)=\frac{9}{2} B V_{0} \alpha^{2} T^{2}+3 N k_{\mathrm{B}} T-T S_{\mathrm{ph}}^{0}(T)-9 N \alpha \gamma k_{\mathrm{B}} T^{2}
$$

First we obtain the equilibrium thermal expansion coefficient, $\alpha$, by calculating

$$
\begin{aligned}
\frac{\partial F(T)}{\partial \alpha}=0 & =9 B V_{0} \alpha T^{2}-9 N \gamma k_{\mathrm{B}} T^{2}, \\
\alpha & =\frac{C_{V} \gamma}{3 B V_{0}},
\end{aligned}
$$

where we have used the classical result $C_{V}=3 N k_{\mathrm{B}} \cdot{ }^{16}$ If the electronic entropy depends on volume, an additional term, $C_{\mathrm{el} V} \gamma_{\mathrm{el}} /\left(3 B V_{0}\right)$ is added to the right-hand side of Eq. 83.

Substituting Eq. 83 for the thermal expansion into 82 for the free energy

$$
F(T)=F^{0}(T)-\frac{9}{2} B V_{0} \alpha^{2} T^{2}
$$

\footnotetext{
16 The effect of phonon energy is small as discussed in Footnote 15, although there is also a small effect from a reduction in zero-point phonon energy as the solid expands.
} 


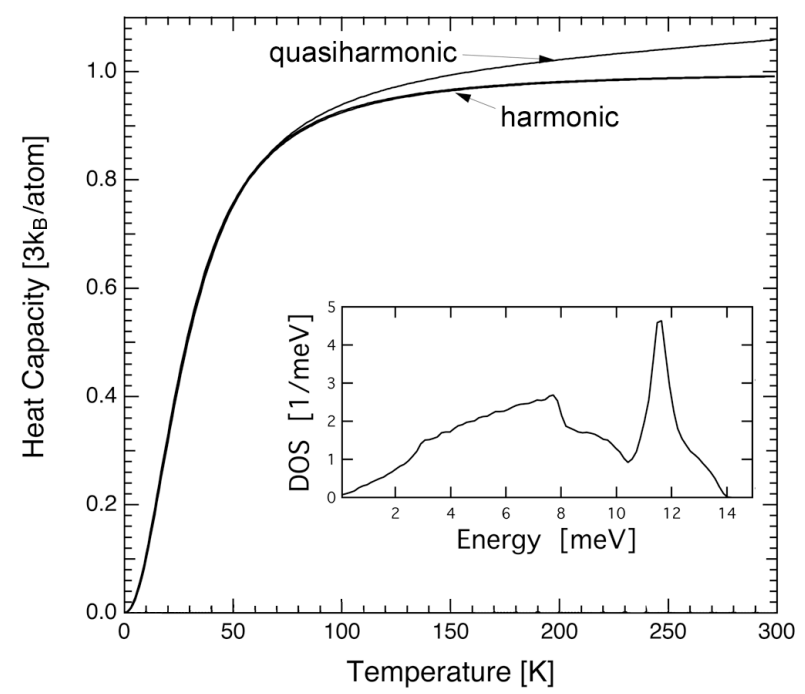

Figure 9. Illustration of a relatively large effect of thermal expansion on the heat capacity, using models of a cerium alloy. Inset is the phonon DOS at $0 \mathrm{~K}$, from which the "harmonic" heat capacity curve was obtained $\left(3 k_{\mathrm{B}}\right.$ /atom is the high-temperature limit of the harmonic model). Using a large $\alpha$ and an experimental $B$ in Eq. 83, a $\gamma$ was obtained from Eq. 83 that predicted the phonon DOS would soften by $6.5 \%$ from 0 to $300 \mathrm{~K}$. The elastic energy from thermal expansion was added to the "harmonic" curve to obtain the "quasiharmonic" curve.

where $F^{0}(T)$ is the harmonic free energy at finite temperature with zero thermal expansion $(\alpha=0)$. We use Eq. 83 to express the quasiharmonic entropy $S_{\mathrm{q}}$ of Eq. 80 or 81 as

$$
S_{\mathrm{q}}=9 B V_{0} \alpha^{2} T
$$

(consistent with differentiating Eq. 84 with respect to $T$ ). Finally, if we substitute Eq. 85 into Eq. 73, we can recover Eq. 74. Typical effects on heat capacity are shown in Fig. 9. This $S_{\mathrm{q}}$ of Eq. 85 should always be considered when comparing the vibrational thermodynamics of different materials at elevated temperatures.

\subsection{Anharmonic Heat Capacity}

In mechanics, the word "anharmonic" describes any oscillator with generalized forces that deviate from linearity with generalized coordinates. In vibrational thermodynamics, "anharmonic" is used more restrictively. The word "quasiharmonic" accounts for the effects of thermal expansion on phonons just considered (e.g., Eqs. $74,85)$, effects that are not strictly harmonic. An implicit assumption of quasiharmonic theory is that an oscillation has a well-defined, long-lived, harmonic-like frequency that changes modestly and predictably with changes in $T$ and $P$.

Equation 83 is useful for separating anharmonic from quasiharmonic behavior. Suppose we know (by inelastic scattering measurements for example) the thermal softening of the phonon modes, from which we calculate an average Grüneisen parameter $\gamma$. With the phonon DOS we can also calculate $C_{V}$ (or its molar quantity), and conventional measurements can provide $B, V_{0}$ (or molar volume $v$ ), and $\alpha$, 
accounting for all unknowns in Eq. 83. If Eq. 83 proves to be true with the $\gamma$ measured from the phonon softening, we say that the solid is "quasiharmonic."

Anharmonic behavior is identified as phonon softening or stiffening that is inconsistent with the Grüneisen parameter $\gamma$ needed for equality in Eq. 83. Anharmonicity involves phenomena beyond those of independent phonons. Higher-order phononphonon interactions are the source of anharmonic behavior as discussed in Sect. 18.2. Section 20 discusses electron-phonon interactions, which also vitiate Eq. $83 .{ }^{17}$ Substantial deviations from quasiharmonic behavior are known for Cr [47], V [48], Ce [49], Mo, W, Si, Ge [50], and anharmonic or non-quasiharmonic effects could be important in many other systems.

\subsection{Thermodynamic Entropy}

The temperature dependence of the entropy

$$
\left.\left.\frac{\mathrm{d} S(T, V)}{\mathrm{d} T}=\frac{\partial S}{\partial T}\right)_{V}+\frac{\partial S}{\partial V}\right)_{T} \frac{\mathrm{d} V}{\mathrm{~d} T}
$$

contains harmonic, quasiharmonic, and anharmonic contributions, along with nonharmonic effects from electron-phonon interactions. For the harmonic part of the vibrational entropy we use Eq. 14, with a phonon DOS, $g_{0}(\varepsilon)$, from low temperature measurement or calculation (ideally $T=0 \mathrm{~K}$ ). The harmonic part contributes to the first term on the right-hand-side of Eq. 86, i.e., $\partial S / \partial T)_{V}$. Harmonic phonons undergo no change in frequency with $T$ or $V$. The temperature dependence of $S_{\mathrm{h}}(T)$ originates with the Planck occupancy factors $n(\varepsilon, T) .{ }^{18}$

Quasiharmonic phonons have frequencies that depend on volume only, and make a contribution through the second term on the right-hand-side of Eq. 86. At a fixed volume, however, they behave as harmonic oscillators. Their frequencies can change with temperature, but only because thermal expansion alters the volume of the solid.

Anharmonic entropy, $S_{\mathrm{a}}$, may contribute to either of the two terms on the righthand side of Eq. 86, but especially the first, a pure temperature-dependence of the vibrational entropy at fixed volume caused by changes in the interatomic force constants with temperature. Consider for example a quartic term in the interatomic potential, which alters the force constants with larger thermal displacements. The quartic term does not affect the thermal expansion, but it does change the phonon frequencies. It causes a type of "phonon-phonon interaction," so called because the existence of phonons affects the energy to create new phonons, as discussed in Section 19. Other temperature-dependences of force constants at constant volume may arise if the nature of the bonding changes as atoms are displaced further from their equilibrium positions, or perhaps thermal excitations of electrons alter the sensitivity of electronic energy to atom displacements (Sections 17, 18.3 and

\footnotetext{
17 It remains a challenge to differentiate between these sources of non-harmonic behavior [46], so sometimes the effects of electron-phonon interactions are called "anharmonic." More often "anharmonic" is reserved for phonon-phonon interactions only.

18 For harmonic solids at high temperatures, differences in the phonon DOS will cause a difference in entropy for different phases, but this difference in entropy does not change with temperature. (At high $T, S$ increases with $T$ by the same amount for each phase.)
} 
20 describe such effects from adiabatic electron-phonon interactions). The second term in Eq. 86 is associated with changes of the interatomic force constants with volume. The quasiharmonic contribution is expected to account for most of it, but the practice of using harmonic entropy expressions, while correct to first order [46], is not fully verified. The cubic term associated with thermal expansion also shortens phonon lifetimes, causing energy spreads of phonon linewidths. This may not alter the vibrational entropy to first order, but again the robustness of the harmonic approximation is not fully tested.

Analogs exist for magnetic dependencies, as can originate from a coupling between magnetic and vibrational degrees of freedom [51]. Invar behavior is an experimental example, but perhaps this is best understood as a volume-dependent electronic transition rather than a magnetic one $[52,53,54]$.

\section{Calorimeters}

The word "calorimeter" covers a broad scope of instruments for measuring large or small transfers of heat to samples at low or high temperatures. From these measurements heat capacity can be determined, or heats of phase transformations or chemical reactions in the sample can be found. ${ }^{19}$ Phase transformations and chemical reactions may involve changes in configurational entropy, however, so for isolating vibrational entropy it is usually better to measure heat capacity data at lower temperatures where no changes in local atomic arrangements are underway. (An exception is the martensitic transformation discussed in Sect. 15.)

Adiabatic calorimeters have performed heat capacity measurements for a century, dating from Nernst and Eucken [55, 56, 57, 58, 59, 60]. The sample and a heater are thermally isolated from their surroundings by vacuum and an adiabatic shield that is maintained at approximately the same temperature as the sample. The latter minimizes radiative heat transfer, and a thin mechanical suspension such as silk thread minimizes heat conduction. Measured electrical energy is provided to the sample through a heater, and the sample temperature is measured by thermometry. After equilibration the temperature rise is measured, from which the heat capacity of the sample and its holder are obtained. Ideally, the sample weight should be large $\left(1 \mathrm{~cm}^{3}\right.$ is relatively small $)$, minimizing background corrections. Heat capacities can be measured to better than $1 \%$ accuracy from typically $10-300 \mathrm{~K}$ with adiabatic calorimeters, but there are many instrument designs and operating ranges. A variation on this method is drift calorimetry. The sample is connected to a thermal bath through a weak thermal link, causing its temperature to drift. Although the rate of drift should be proportional to the heat capacity of the sample, more accurate data are usually obtained by applying pulses of electrical energy, and identifying the extra temperature change over the baseline drift. Another approach is used for heat pulse, or relaxation calorimeters, which also have thermal links between the sample assembly and a thermal anchor. After a pulse of electrical energy raises the temperature of the sample, its temperature decays towards that of the thermal

\footnotetext{
19 Proposals appear with some regularity to use accurate heat capacity data to determine the phonon DOS. These prove to be too optimistic, although some lower moments of the phonon DOS can be determined this way.
} 
anchor. The rate of decay (sometimes modeled as an exponential function or a sum of two exponentials) is used to obtain the heat capacity of the sample, which can be proportional to the characteristic time of the decay [61,62, 63, 64]. A related technique, which also has advantages for small samples, is to apply an oscillating heating signal to the sample, and measuring the thermal response at the frequency of the heating signal using a lockin amplifier to minimize noise [65].

Differential measurements can be highly accurate, and are sometimes useful for measuring differences in vibrational entropy of different materials. A differential scanning calorimeter heats two samples, perhaps matched in their composition and mass, but with different crystal structures. The electrical heating is performed with feedback control so that the temperatures of both samples increase at the same rate. Differences in heat capacity are measured by the differences in electrical power required to maintain the same heating rates. This method of differential scanning calorimetry has, in principle, the advantage that it nulls the background from the sample holder, but background issues prove difficult when highly accurate measurements are required. The method also has limitations in the range of temperatures that can be achieved, because if there is no adiabatic isolation, the electronic heating system needs to provide considerably higher power at higher temperatures. A simpler differential measurement technique, differential thermal analysis, places two samples in a common thermal environment, such as side-by-side in a furnace. The furnace temperature is increased uniformly, and the temperature difference between the two samples is measured to obtain their differential heat capacity. These differential measurements can be accurate to $2 \%$ of the total heat capacity, but like all methods of calorimetry, it is a challenge to obtain high-quality results over a wide range of temperatures.

\section{Neutron Scattering}

\subsection{Elastic and Inelastic Scattering}

Figure 10 shows the geometry, wavevectors and position vectors for neutron scattering. The plane wave incident from the left, $\Psi_{\text {in }}\left(\vec{r}^{\prime}, t\right)$, has the form

$$
\Psi_{\text {in }}\left(\vec{r}^{\prime}, t\right)=\mathrm{e}^{\mathrm{i}\left(\vec{k}_{\mathrm{i}} \cdot \vec{r}^{\prime}-\omega_{\mathrm{i}} t\right)} .
$$

For scattering from one nucleus (which is essentially a point compared to typical neutron wavelengths), a spherical wave, $\Psi_{\mathrm{sc}}(\vec{r}, t)$, emerges from $\vec{r}^{\prime}$, and reaches the detector at $\vec{r}$ at time $t^{\prime}>t$

$$
\Psi_{\mathrm{sc}}\left(\vec{r}, t^{\prime}\right)=\frac{-1}{4 \pi} \frac{\mathrm{e}^{\mathrm{i}\left[k_{\mathrm{f}}\left|\vec{r}-\vec{r}^{\prime}\right|-\omega_{\mathrm{f}}\left(t^{\prime}-t\right)\right]}}{\left|\vec{r}-\vec{r}^{\prime}\right|} .
$$

The difference, $\vec{r}-\vec{r}^{\prime}$, is the distance from the nucleus to the detector. Because Eq. 88 gives the contribution to the wavefunction at position $\vec{r}$ caused by a $\delta$-function at $\vec{r}^{\prime}$, with its prefactor it is the Green's function of the Schrödinger equation of the neutron scattering problem [34].

In the first Born approximation [34], the amplitude of the scattered wave at the detector, $\Psi_{\mathrm{sc}}\left(\vec{r}, t^{\prime}\right)$, is proportional to the product of: 1$)$ the amplitude of the 


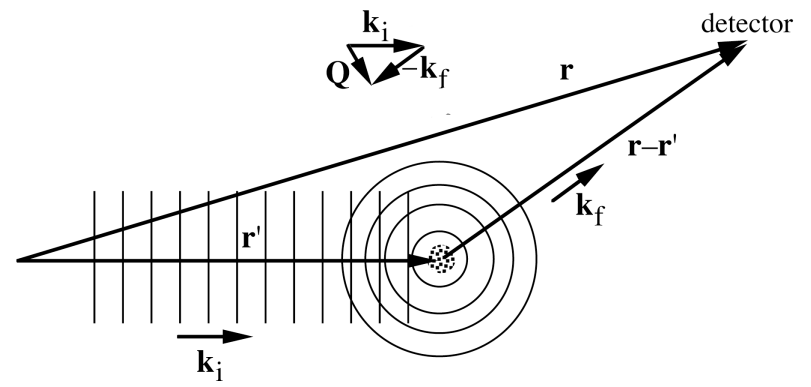

Figure 10. Wavevectors and position vectors for neutron scattering.

incident wave, $\Psi_{\text {in }}$ (Eq. 87), 2) the Green's function of Eq. 88, and 3) the potential that scatters the neutron out of its initial state

$$
\Psi_{\mathrm{sc}}\left(\vec{r}, t^{\prime}\right)=\frac{-m_{\mathrm{n}}}{2 \pi \hbar^{2}} \int V_{\mathrm{n}}\left(\vec{r}^{\prime}\right) \mathrm{e}^{\mathrm{i}\left(\vec{k}_{\mathrm{i}} \cdot \vec{r}^{\prime}-\omega_{\mathrm{i}} t\right)} \frac{\mathrm{e}^{\mathrm{i}\left[k_{\mathrm{f}}\left|\vec{r}-\vec{r}^{\prime}\right|-\omega_{\mathrm{f}}\left(t^{\prime}-t\right)\right]}}{\left|\vec{r}-\vec{r}^{\prime}\right|} \mathrm{d}^{3} \vec{r}^{\prime} .
$$

For nuclear scattering, relevant to phonon measurements, we use the "Fermi pseudopotential," $V_{\text {nuc }}\left(\vec{r}^{\prime}\right)=4 \pi \hbar^{2} /\left(2 m_{\mathrm{n}}\right) b \delta\left(\vec{r}^{\prime}\right)$ which places all the potential at the point nucleus, where $b$ is a fixed length. ${ }^{20}$ Fermi pseudopotentials are placed at crystal translation vectors, $\left\{\vec{R}_{j}\right\}$, accounting for all $N$ nuclei in the crystal

$$
V_{\mathrm{n}}\left(\vec{r}^{\prime}\right)=4 \pi \frac{\hbar^{2}}{2 m_{\mathrm{n}}} \sum_{j=1}^{N} b_{j} \delta\left(\vec{r}^{\prime}-\vec{R}_{j}\right) .
$$

This sum of $\delta$-functions transforms the integration of Eq. 89 into a sum over points at $\vec{R}_{j}$. For laboratory distances (large compared to the size of the scatterer), $\vec{r} \gg \vec{R}_{j}$, the outgoing spherical wave can be treated as a plane wave, allowing rearrangement

$$
\Psi_{\mathrm{sc}}\left(\vec{r}, t^{\prime}\right)=\frac{\mathrm{e}^{\mathrm{i}\left(\vec{k}_{\mathrm{f}} \cdot \vec{r}-\omega_{\mathrm{f}} t^{\prime}\right)}}{|\vec{r}|} \sum_{j} b_{j} \mathrm{e}^{\mathrm{i}\left(\vec{k}_{\mathrm{i}}-\vec{k}_{\mathrm{f}}\right) \cdot \vec{R}_{j}} \mathrm{e}^{\mathrm{i}\left(\omega_{\mathrm{f}}-\omega_{\mathrm{i}}\right) t} .
$$

The factor $\mathrm{e}^{\mathrm{i}\left(\vec{k}_{\mathrm{f}} \cdot \vec{r}-\omega_{\mathrm{f}} t^{\prime}\right)}$ gives the precise phase at the time and place of neutron detection. It has modulus unity, and we ignore it because we measure the intensity of scattering. The relative phases of waves from all nuclei are essential, however, because they account for interferences.

We define the change in neutron wavevector, $\vec{Q} \equiv \vec{k}_{\mathrm{i}}-\vec{k}_{\mathrm{f}}$ (and the momentum transfer from the neutron to the sample is $\hbar \vec{Q}$ ). Using Eq. 18 for $\vec{R}_{j}$, and allowing the scattered wave to emanate from the nucleus at later times

$$
\Psi_{\mathrm{sc}}(\vec{r})=\frac{1}{r} \sum_{l, \kappa} b_{\kappa} \mathrm{e}^{\mathrm{i} \vec{Q} \cdot\left(\vec{r}_{l}+\vec{r}_{\kappa}\right)} \mathrm{e}^{\mathrm{i} \vec{k}_{\mathrm{i}} \cdot \vec{u}_{l \kappa}(0)} \mathrm{e}^{-\mathrm{i} \vec{k}_{\mathrm{f}} \cdot \vec{u}_{l \kappa}(t)} \mathrm{e}^{\mathrm{i}\left(\omega_{\mathrm{f}}-\omega_{\mathrm{i}}\right) t},
$$

where we note that because all unit cells are identical, $b$ depends on the basis vector only. It is easiest to understand the case when the scattering is a fast process, such as

\footnotetext{
20 Typically of a nuclear scale of $10^{-12} \mathrm{~cm}$ or so, sometimes with an imaginary component to account for absorption.
} 
for x-ray scattering, where an x-ray traverses the crystal more rapidly than typical vibrational periods of atoms. Incoherent neutron scattering is also fast, where the thermal neutron is scattered from individual atomic nuclei without concern for phase relationships between nuclei. This motivates a first approximation; ignore the time dependence of the potential by setting $\vec{u}_{l \kappa}(t)=\vec{u}_{l \kappa}(0)$, and take the time average

$$
\begin{aligned}
& \Psi_{\mathrm{sc}}^{\mathrm{el}}(\vec{Q}, \vec{r})=\frac{1}{r} \sum_{l, \kappa} b_{\kappa} \mathrm{e}^{\mathrm{i} \vec{Q}_{\left(\vec{r}_{l}+\vec{r}_{\kappa}\right)}} \mathrm{e}^{\mathrm{i} \vec{Q} \cdot \vec{u}_{l \kappa}(0)} \frac{1}{\tau} \int_{0}^{\tau} \mathrm{e}^{\mathrm{i}\left(\omega_{\mathrm{f}}-\omega_{\mathrm{i}}\right) t} \mathrm{~d} t, \\
& \Psi_{\mathrm{sc}}^{\mathrm{el}}(\vec{Q}, \vec{r})=\frac{1}{r} \sum_{l, \kappa} b_{\kappa} \mathrm{e}^{\mathrm{i} \vec{Q} \cdot\left(\vec{r}_{l}+\vec{r}_{\kappa}\right)} \mathrm{e}^{\mathrm{i} \vec{Q} \cdot \vec{u}_{l \kappa}(0)} \delta\left(\omega_{\mathrm{f}}-\omega_{\mathrm{i}}\right),
\end{aligned}
$$

The $\delta$-function enforces no change of frequency or energy, so Eq. 94 pertains to elastic scattering, where the modes of atom vibrations are unchanged. We need to perform an ensemble average over the different values of $\vec{u}_{l \kappa}(0)$, which evaluates as ${ }^{21}$

$$
\left\langle\mathrm{e}^{\mathrm{i} \vec{Q} \cdot \vec{u}(0)}\right\rangle_{N}=\mathrm{e}^{-\frac{1}{2}\left\langle[\vec{Q} \cdot \vec{u}(0)]^{2}\right\rangle_{N}} \equiv \mathrm{e}^{-W} .
$$

Equation 94 for elastic scattering becomes

$$
\Psi_{\mathrm{sc}}^{\mathrm{el}}(\vec{Q}, \vec{r})=\frac{\mathrm{e}^{-W}}{r} \sum_{\kappa} b_{\kappa} \mathrm{e}^{\mathrm{i} \vec{Q} \cdot \vec{r}_{\kappa}} \sum_{l} \mathrm{e}^{\mathrm{i} \vec{Q} \cdot \vec{r}_{l}} \equiv \frac{\mathrm{e}^{-W}}{r} \mathcal{F}(\vec{Q}) \mathcal{S}(\vec{Q}) .
$$

Here we separated the sums over basis and lattice vectors into a structure factor, $\mathcal{F}(\vec{Q})$, and a shape factor, $\mathcal{S}(\vec{Q})$. Equation 96 can be used to calculate the neutron diffraction pattern from a crystal, giving the average static structure. The factor $\mathrm{e}^{-W}$ is the "Debye-Waller factor," which becomes appreciably less than 1 when the atom displacement $\vec{u}_{\kappa}$ is comparable to $1 / Q \sim \lambda / 2 \pi$, the wavelength associated with the scattering angle and energy. This is consistent with the origin of the Debye-Waller factor from the destructive interference of scattered wavelets that are emitted from different spatial positions of the displaced nuclei. Note that the ensemble average of Eq. 95 does not reveal the energy of an atom vibration, only how displaced atom positions alter the phase.

Next consider a process of scattering that is slow compared to the atomic vibrational period. Such is the case for coherent scattering of neutrons by phonons, and for nuclear resonant scattering of photons by phonons. For clarity, we consider the scattering by one phonon of wavevector $\vec{k}$. Equation 28 shows that $\vec{u}_{l \kappa \vec{k}}(t)=$ $\vec{u}_{l \kappa \vec{k}}(0) \mathrm{e}^{-\mathrm{i} \omega_{\vec{k}} t}$. We need to take a time average of the displacement factors in Eq. 92

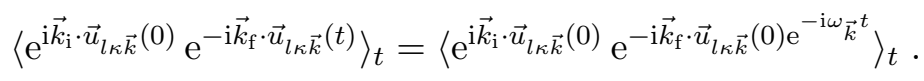

Coherent phonon scattering involves correlations of displacements $\vec{u}_{l \kappa \vec{k}}(t)$ at different atom positions at different times. The intensity of the coherent inelastic

\footnotetext{
21 An approximate demonstration of this is by expanding $\exp (\mathrm{i} \vec{Q} \cdot \vec{u})=1+\mathrm{i} \vec{Q} \cdot \vec{u}+\ldots$ and taking either an ensemble average or a time average. The average of the second term, $\mathrm{i} \vec{Q} \cdot \vec{u}$ is zero owing to positive and negative excursions of $\vec{u}$, leaving the quadratic term, consistent with Eq. 95. The average of the subsequent cubic term also vanishes, conveniently, but unfortunately the quartic term has the wrong sign. Nevertheless, it can be proved that Eq. 95 is exact if the probability distribution of $\vec{u}$ is Gaussian about the origin, and this is often reasonable for lattice dynamics.
} 
scattering proves to be the Fourier transform of the Van Hove space-time correlation function [66], and is a rich approach to studying dispersive excitations in solids. The proper quantum mechanical treatment of time and temperature averages is beyond the scope of this review, but Eq. 98 below is the proper result for incoherent scattering. Furthermore, it is possible to average the coherent scattering over all $\vec{Q}$ to recover this incoherent result $[67,68,69]$, and the incoherent approximation serves us well in the following analysis of multiphonon scattering. The loss of phase information by averaging the coherent scattering over all $\vec{Q}$ brings us back to consideration of a fast scattering process.

\subsection{One-Phonon And Multiphonon Scattering}

Equation 96 is the elastic scattering only. It does not conserve the nuclear scattering cross section. Nuclear interactions do not necessarily depend on the displacements of atoms, so we need a compensating factor $\mathrm{e}^{+\left\langle(\vec{Q} \cdot \vec{u})^{2}\right\rangle}$ to conserve the cross-section and account for the rest of the scattering intensity. Expanding this new factor, we calculate the intensity as $\Psi_{\mathrm{sc}}^{*} \Psi_{\mathrm{sc}}$, and for clarity we normalize the intensity

$$
\begin{aligned}
\left\langle\Psi_{\mathrm{sc}}^{*} \Psi_{\mathrm{sc}}\right\rangle_{N} & =\frac{1}{\mathcal{R}} \sum_{\kappa}^{\mathcal{R}} \mathrm{e}^{-\left\langle\left(\vec{Q} \cdot \vec{u}_{\kappa}\right)^{2}\right\rangle} \\
& \times\left(1+\left\langle\left(\vec{Q} \cdot \vec{u}_{\kappa}\right)^{2}\right\rangle+\frac{1}{2}\left(\left\langle\left(\vec{Q} \cdot \vec{u}_{\kappa}\right)^{2}\right\rangle\right)^{2}+\ldots\right)
\end{aligned}
$$

The first and second terms in parentheses of Eq. 98 are the fractions of elastic $(0-$ phonon) and 1-phonon scattering. The third term with the $\frac{1}{2}\left(\left\langle\left(\vec{Q} \cdot \vec{u}_{\kappa}\right)^{2}\right\rangle\right)^{2}$ accounts for 2-phonon scattering processes. Only the 1-phonon scattering proves useful for obtaining a phonon DOS, but it is important to have an understanding of the higher-order terms (called "multiphonon scattering") for designing an experiment, or performing quantitative corrections of experimental data.

A more detailed treatment of coherent 1-phonon scattering includes the thermal factors for phonon populations, giving the double-differential scattering cross section $^{22}$

$$
\begin{aligned}
\frac{\mathrm{d}^{2} \sigma}{\mathrm{d} \Omega \mathrm{d} E}=\frac{k_{\mathrm{f}}}{k_{\mathrm{i}}} \frac{(2 \pi)^{3}}{2 V_{0}} & \sum_{\vec{g}, \vec{k}} \frac{n(\vec{k})+\frac{1}{2} \pm \frac{1}{2}}{\omega_{\vec{k}}} \\
& \times\left|\sum_{\kappa} \frac{b_{\kappa}}{\sqrt{m_{\kappa}}} \mathrm{e}^{-\left\langle\left(\vec{Q} \cdot \vec{u}_{\kappa}\right)^{2}\right\rangle}\left[\vec{Q} \cdot \vec{e}_{\kappa}(\vec{k})\right] \mathrm{e}^{\mathrm{i} \vec{Q} \cdot \vec{r}_{\kappa}}\right|^{2} \\
& \times \delta(\vec{Q}+\vec{k} \pm \vec{g}) \delta\left(\omega-\omega_{\vec{k}}\right),
\end{aligned}
$$

where the phonon occupancy factor, $n(\vec{k})$, is the Planck distribution of Eq. 7 at the temperature of interest and $\varepsilon_{i}=\hbar \omega_{\vec{k}}$. The signs in the factor $n(\vec{k})+\frac{1}{2} \pm \frac{1}{2}$ in Eq. 99 are such that it is $n(\vec{k})$ for phonon annihilation, and $n(\vec{k})+1$ for phonon creation - it

\footnotetext{
${ }^{22}$ A new factor is the ratio $k_{\mathrm{f}} / k_{\mathrm{i}}$, which expresses the effect on flux caused by the rate at which neutrons leave the sample. Compared to an elastically-scattered neutron, fewer neutrons per second will pass into an increment of solid angle $\mathrm{d} \Omega$ if they are slowed to smaller values of $k_{\mathrm{f}}$. Also, the use of solid angle eliminates the need for the factor $\mathrm{r}^{-2}$.
} 
is always possible to create a phonon, even at $T=0$ when no phonon excitations are present. The $\delta(\vec{Q}+\vec{k} \pm \vec{g})$ and $\delta(\omega-\omega(\vec{k}))$ enforce the conservation of momentum and energy on the neutron scattering process. The phonons on different branches must be considered separately in Eq. 99, and it is traditional to add a "branch index," sometimes denoted $j$, to $\omega(\vec{k})$, since more than one $\omega_{j}$ may correspond to a specific $\vec{k}$ (cf., Eq. 29).

Why should the expansion of an evidently classical exponential in Eq. 98 produce the series of neutron-phonon interactions? In the classical limit, the scattering of every neutron is inelastic, generating heat composed of a number of phonons with total energy, $E$, the energy transfer from the neutron. For classical scattering, an atom of mass $m$ will recoil with velocity $v=\hbar Q / m$, with momentum transfer $\hbar Q$, and energy transfer

$$
E_{R}=\frac{\hbar^{2} Q^{2}}{2 m}
$$

In the quantum limit where $Q$ is small, the energy recoil may or may not occur, depending on whether phonons are created or not. The total scattering, $S(Q, E)$, is the sum of components, $\sum_{n=0}^{\infty} S_{n}(Q, E)$, from neutrons scattered after creating different numbers, $n$, of phonons in the sample. ${ }^{23}$ The thermal energy is

$$
k_{\mathrm{B}} T=2 m \omega^{2}\left\langle u^{2}\right\rangle
$$

for one harmonic mode of frequency $\omega$. From Eqs. 100 and 101

$$
Q^{2}\left\langle u^{2}\right\rangle=\frac{E_{\mathrm{R}}}{\hbar \omega} \frac{k_{\mathrm{B}} T}{\hbar \omega}
$$

This is the quantity in which the multiphonon expansion is performed (Eq. 98). From Eq. 102 we see that the relative intensities of the sequence of phonon scattering terms scale with 1) the ratio of the recoil energy to the oscillator energy, and 2) the ratio of temperature to the oscillator energy. When the first ratio is small, quantum mechanics requires that some recoils excite the oscillator, but most do not. The same is true for temperature (and at low temperatures we of course have low heat capacity because the oscillator is usually not excited).

It remains to get the spectral shape of each order of the multiphonon scattering. The spectrum for one-phonon scattering weights more heavily the low-energy modes because they have larger amplitudes of motion, providing a factor of $g(\varepsilon) / \varepsilon$. The number of phonons is the Planck distribution $1 /\left[\exp \left(\varepsilon / k_{\mathrm{B}} T\right)-1\right]$, and the two factors provide the shape of the 1-phonon profile, $A_{1}(\varepsilon)$ :

$$
A_{1}(\varepsilon)=\frac{g(\varepsilon)}{\varepsilon} \frac{1}{\mathrm{e}^{\varepsilon / k_{\mathrm{B}} T}-1} .
$$

When two phonons are created simultaneously, the total spectrum of energies is the convolution of the 1 -phonon profile with the 1 -phonon profile. ${ }^{24}$ The $n$-phonon

\footnotetext{
23 Phonon annihilation is handled by extending the range of $E$ to negative values for each $S_{n}(Q, E)$.

${ }_{24}$ Consider each phonon excitation to be a random variable with a probability distribution of $A_{1}$. The sum of two random variables has a distribution that is the convolution of the probability distributions $A_{1} * A_{1}$.
} 
profile is the convolution of another 1-phonon profile with the $(n-1)$-phonon profile

$$
A_{n}(\varepsilon)=A_{1} * A_{n-1}=\int_{-\infty}^{\infty} A_{1}\left(\varepsilon-\varepsilon^{\prime}\right) A_{n-1}\left(\varepsilon^{\prime}\right) \mathrm{d} \varepsilon^{\prime} .
$$

Starting with $A_{1}$, we can generate the spectral shapes of all orders of multiphonon scattering by the systematic application of Eq. $104 .{ }^{25}$ The total scattering is the sum of these spectral profiles, weighted by the corresponding terms of Eq. 98

$$
S(Q, E)=\sum_{n=0}^{\infty} \exp (-2 W) \frac{(2 W)^{n}}{n !} A_{n}(E),
$$

where we have defined $S(Q, E)$ as the double-differential cross-section $\mathrm{d}^{2} \sigma / \mathrm{d} \Omega \mathrm{d} E$ without prefactors. ${ }^{26}$

In much experimental work, we seek to isolate the single scattering profile, $A_{1}(E)$, from the measured $S(Q, E)$, and then determine the phonon DOS $g(E)$ by Eq. 103. One way to correct for multiphonon scattering is by iteration on an initial guess of the phonon DOS. With an initial guess at the DOS or $A_{1}(E)$ (often the $S(Q, E)$ with a constant background removed), the various $A_{2}(E), A_{3}(E), A_{4}(E) \ldots$ are calculated and subtracted from $S(Q, E)$ to give a better $A_{1}(E)$. Iteration is complete when there is a good match between the measured $S(Q, E)$ and the $S(Q, E)$ recalculated with Eq. 105. When the multiphonon scattering is weak, perhaps only two iterations are necessary. When the multiphonon scattering is strong, the procedure becomes difficult and slow. If there is strong multiphonon scattering, however, the experiment is moving into the limit of classical scattering, so isolating the single phonon spectrum becomes more challenging.

\subsection{Neutron Weighting}

Strictly speaking, the phonon DOS from the procedure outlined above is not the true phonon DOS, but rather the neutron-weighted DOS. For an elemental scatterer, the neutron-weighted DOS is identical to the phonon DOS. This is not the usual case for a sample that contains more than one type of atom. The neutron-weighted phonon DOS is

$$
g_{\mathrm{NW}}(\varepsilon) \propto \sum_{d} g_{d}(\varepsilon) \exp \left(-2 W_{d}\right) \exp (2 W) \frac{\sigma_{d}}{m_{d}}
$$

where the $g_{d}(\varepsilon)$ are the phonon partial DOS functions of Eq. 34. The Debye-Waller factor is an explicit function of $g_{d}(\varepsilon)$. The term $\exp (2 W)$ is the average Debye-Waller

\footnotetext{
25 A subtlety arises for coherent scattering from crystals that are sufficiently anharmonic so that the phonon lifetime is shorter than the characteristic time for neutron scattering. When, for example, one phonon decays into two during the scattering, there can be constructive or destructive interference between the neutron wavepackets scattered by a one-phonon process and a two-phonon process. This interference can alter the spectral shape. Although recognized some time ago [70], identifying this effect has been a challenge [71, 72, 73].

26 This $S(Q, E)$, called the "scattering law," is normalized so that it is the double Fourier transform of the Van Hove correlation function $G(\vec{r}, t)$.
} 
correction; it is calculated from the self-consistent neutron-weighted DOS. At low temperatures, the factor $\exp \left[2\left(W-W_{d}\right)\right]$ is approximately unity.

For the case where $\sigma_{d} / m_{d}$ is the same for all species $d, g_{\mathrm{NW}}(\varepsilon) \approx g(\varepsilon)$, but in general obtaining the true phonon DOS from the neutron-weighted phonon DOS requires a full analysis of the lattice dynamics. This can be performed by simulational procedures or by some types of analytical methods. These are important analyses, but are mostly beyond the scope of this review. One example is presented in Section 13.2 .

\subsection{Direct-Geometry Fermi Chopper Spectrometer}

Chopper spectrometers have evolved with advances in electronics $[74,75,76,77,78]$. A Fermi chopper is a fast shutter located a fixed distance from the neutron source. By aligning a slot at the right time after a neutron burst leaves the moderator source, it selects neutrons having a particular velocity, and hence a particular neutron energy $E=\frac{1}{2} m v^{2}$. Its operation is best illustrated with the distance versus time diagram of Fig. 11. The slopes of the lines are neutron velocities. Neutrons of many velocities are emitted from the moderator. The figure depicts two neutron pulses from the moderator, separated in time by perhaps $1 / 60$ sec. The Fermi chopper selects a narrow range of velocities, corresponding to a time window of tens of microseconds. Of course the narrower the window, the more neutrons are blocked by the chopper, and the lower the flux of neutrons on the sample. On the other hand, the narrower the window, the more precisely selected are the velocities, and hence better energy resolution is achieved. The Fermi chopper therefore controls the incident energy, the intensity, and the energy resolution of the neutrons incident on the sample. ${ }^{27}$

The sample usually transmits most of the incident neutrons without energy change, and most of the scattered neutrons are scattered elastically. There is a strong tendency for the lines in Fig. 11 to have unchanged slopes through the sample. Neutrons that are scattered inelastically, however, have lines with kinks at the sample, and have slopes after the sample that are either steeper or shallower than for the incident beam.

A Fermi chopper spectrometer works by timing. The time of detection of the elastic neutrons is known from the velocity selected by the Fermi chopper, and the elastically-scattered neutrons give an intense peak in the time spectra from the detectors. The neutrons that arrive earlier have gained energy from the sample, and those that arrive later have lost energy to the sample. The neutron energy spectrum is obtained from the histogram of neutron arrival times. From the energy transfer and the scattering angle, the momentum transfer can be determined.

\subsection{Triple-Axis Spectrometer}

The triple-axis spectrometer, invented by Brockhouse [79, 80, 81], uses a steady beam of neutrons from the core of a nuclear reactor. After the neutrons are moderated to thermal energies, a monochromator crystal selects neutrons of a particular energy

\footnotetext{
27 The moderator also affects energy resolution. Figure 11 shows emission of neutrons at two points in time, but there is actually a spread of emission times, especially for neutrons of lower energy.
} 


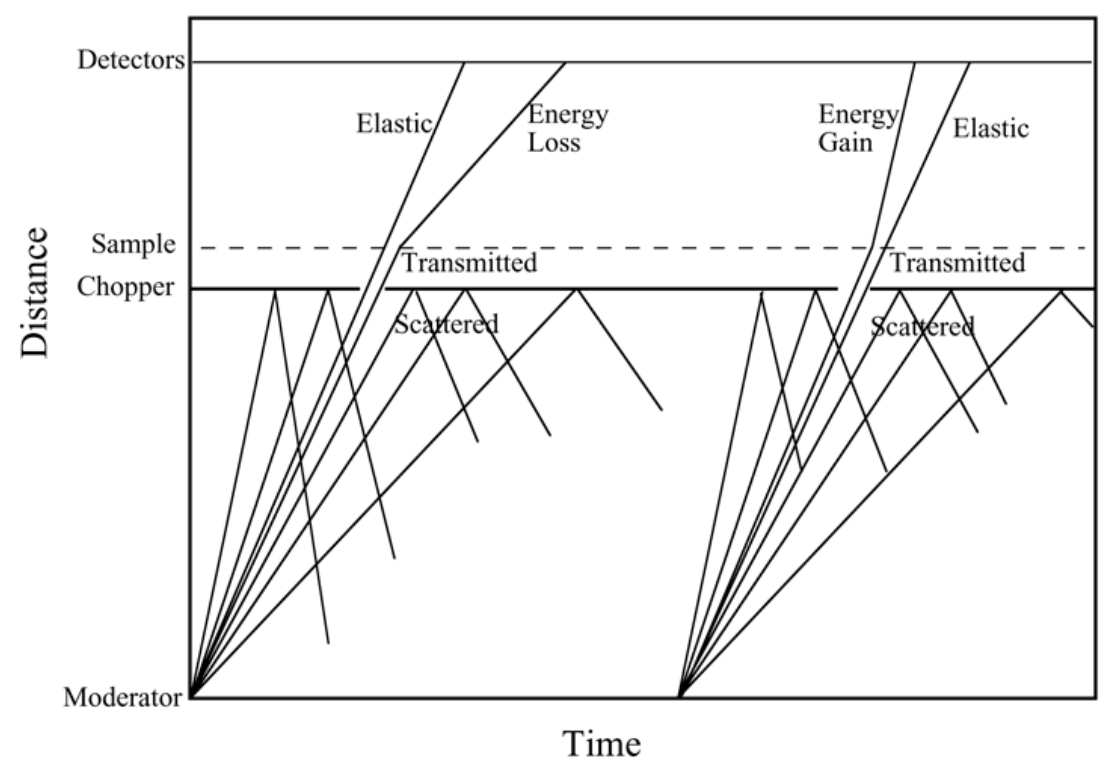

Figure 11. Distance-time diagram for inelastic scattering by a direct geometry chopper spectrometer. Positions of the moderator, chopper, sample, and detectors are marked on the vertical axis. Two moderator pulses occur at points on the $x$-axis.

and sends them in a beam to the sample. The sample is typically a single crystal, positioned with different orientations with respect to the incident beam. An analyzer crystal is used to select an energy of the neutrons leaving the sample, and directs the final beam into a detector. There are a number of modes of operation of a triple-axis instrument, but for measuring phonon dispersions along crystallographic directions it is typical to operate the instrument with a fixed momentum transfer, $\vec{Q}$. The final energy can be selected by fixing the angle of the analyzer crystal with respect to the sample and detector. The energy transfer is varied by changing the orientation of the monochromator crystal, and moving the sample to remain in the incident beam. Maintaining a fixed momentum transfer requires changing the orientation of the analyzer system around the sample. A computer system is used to control the scan over $E$ at constant $\vec{Q}$.

The triple-axis spectrometer selects one value of $E$ and one value of $\vec{Q}$ at any one time. It is the instrument of choice for measurements on single crystals, where it is important to measure dispersions of excitations along crystallographic directions. Single-crystal measurements of phonon dispersion curves can be interpreted directly when phonon peaks are evident above the background. Fitting the dispersion curves to Born-von Kármán models has been an important method for determining interatomic force constants. With these force constants it is then possible to calculate the energies of phonons of other $\vec{k}$, and obtain a phonon DOS. 


\section{Ab-Initio Methods}

\subsection{Density Functional Theory}

A large branch of computational materials science owes its predictive power to developments in density functional theory (DFT), in conjunction with advances in computing power. There are excellent reviews and books on density functional theory and its applications to materials, some of which offer a broader context of condensed matter theory $[82,83,84,85]$. This rich field is far too extensive to cover here. The techniques for calculating phonons from first principles also deserve a much more extensive presentation $[9,86,87,88,89,90,91,92,93,94]$. This brief overview of some key methods and concepts is presented with apologies that numerous intellectual achievements in this rapidly-moving field cannot be covered.

Density functional theory elevates the importance of the electron density with respect to the electron wavefunctions, relying on a theorem that all properties of an electronic system can be obtained from the ground state electron density [95, 96]. A functional relationship must exist between the density and the physical quantity of interest, but finding this relationship is a challenge. The successful approach developed by Kohn and Sham [97] assumes that the ground state electron density of a system of interacting electrons can be equal to that of a non-interacting system of electrons, with an interacting electron density. Working with independent electrons makes calculations practical, freeing us from time-ordered-fermion field operators for electron-electron interactions. In particular, the electron exchange and Coulomb correlation energy, which are nonlocal multibody interactions, can be successfully approximated with functionals of the local density, or the density and its local gradients, for example. The density is obtained from the electron wavefunctions, so computational algorithms require iteration to self consistency of the potential and the wavefunctions. The local density functional theory has been surprisingly successful, although problems occur for strongly-correlated electron systems.

\subsection{First-Principles Phonon Calculations}

Principles for calculating phonon dispersions from the electronic energy were outlined in the classic work of Ziman [98] and others [99]. Two approaches are used. The direct methods impose displacements of atoms, and calculate energies of distorted configurations. In the supercell method, pairs of atoms are displaced by $\vec{u}$ and $\vec{u}^{\prime}$ along directions of symmetry, for example, and from the energies of the distorted structures, $E\left(\vec{u}, \vec{u}^{\prime}\right)$, the force constants $\underline{\Phi}_{l \kappa l^{\prime} \kappa^{\prime}}$ of Eq. 27 can be calculated as

$$
\left.\Phi_{\alpha \alpha^{\prime} l \kappa l^{\prime} \kappa^{\prime}}=\frac{\partial^{2} E\left(\vec{u}, \vec{u}^{\prime}\right)}{\partial u_{\alpha l \kappa} \partial u_{\alpha^{\prime} l^{\prime} \kappa^{\prime}}}\right)_{0},
$$

(cf., Eq. 21). With these ab-initio force constants, the lattice dynamics can be calculated [88]. The Hellman-Feynman theorem shows that errors in the wavefunctions that cause only second-order errors in energy are responsible for first-order errors in forces, so energy convergence is stringent $[9,92]$. With the frozen phonon method, the potential energy of an individual phonon is calculated directly by generating periodic 
distortions of a cell or supercell [100, 101]. Calculations with direct methods are versatile, and can account for unusual structures. Frozen phonons of different amplitudes are a natural way to account for anharmonic potential energies [102, 103, 104], although a good sampling of all phonons is challenging. When periodic boundary conditions are used, the cell should be comfortably larger than the longest-range interatomic force, and the phonon wavevector should be commensurate with the structure. With many atoms and electrons in a large supercell, however, the calculations can become unwieldy, especially since the computation time often scales as the cube of the number of electrons.

The second approach calculates the sensitivities to small density modulation, usually with a linear response theory $[86,87,89,105]$. These are second derivatives of the energy, and the simplest approach to the problem is with second order perturbation theory. For periodic crystals with both electrons and phonons expressed as Bloch functions, the density susceptibility function for the Fourier coefficient $(\vec{k})$ of the electron density depends on the Fourier coefficient of the phonon modulation $\left(\vec{k}^{\prime}\right)$ as

$$
\chi\left(\vec{k}, \vec{k}^{\prime}\right)=2 \sum_{i} \sum_{j} \frac{\left\langle i\left|\mathrm{e}^{-\mathrm{i} \vec{k} \cdot \vec{r}}\right| j\right\rangle\left\langle j\left|\mathrm{e}^{\mathrm{i} \vec{k}^{\prime} \cdot \vec{r}}\right| i\right\rangle}{\epsilon_{i}-\epsilon_{j}},
$$

where the intermediate electronic states $|j\rangle$ are unoccupied, and the states $|i\rangle$ are occupied. A considerable simplification is that $\vec{k}$ and $\vec{k}^{\prime}$ must be related by a reciprocal lattice vector, reducing the number of terms in Eq. 108, although for more realistic interacting systems, the situation is not so simple. A variational approach for obtaining the energy to second order has been developed to obtain a linear response to periodic perturbations [89]. In this method, the dynamical matrix can be calculated directly [90]. It is also possible to recover real-space force constants by Fourier transformation of the dynamical matrix for appropriate selections of $\vec{k}$.

\subsection{Molecular Dynamics}

Molecular dynamics simulations integrate equations of motion to follow in time the positions and velocities of all atoms. Many molecular dynamics simulations are classical or semi-classical, using forces obtained as gradients of model potential energy functions, for example. The atoms are allowed to move under Newton's law for a short time, and the forces are recalculated for the new configuration of atoms. These simulations can be extended beyond a microcanonical ensemble by allowing contact of a small simulation system with a reservoir, accounting for fluctuations of temperature or pressure, for example. Realistic thermostatic or barostatic control can be achieved by extending the dynamical system with degrees of freedom that represent the external system [106, 107]. Molecular dynamics is not restricted to harmonic forces, and large-amplitude displacements allow investigations of anharmonic behavior. Accurate force fields that account for all detail of the local atomic environment can be a challenge. Such force fields can be obtained from quantum mechanics, but often they are empirical. 
The velocity-velocity autocorrelation function $\langle v(t) \mid v(0)\rangle$ can be calculated from the trajectory of each atom in a molecular dynamics simulation. ${ }^{28}$ The Fourier transform of the velocity-velocity autocorrelation function gives the phonon DOS [108]

$$
g(\omega)=\int \mathrm{e}^{\mathrm{i} \omega t} \frac{\langle v(t) \mid v(0)\rangle}{\langle v(0) \mid v(0)\rangle} \mathrm{d} t .
$$

In another approach, it is possible to obtain differences in thermodynamic quantities such as phonon free energy by parameterizing a Hamiltonian to allow the system to switch from one structure to another, and integrating along the path of this switching parameter.

It is possible to solve for the forces on the moving nuclei by performing a full DFT calculation at each timestep of the simulation, but this is cumbersome. The Car-Parrinello algorithm makes practical quantum molecular dynamics simulations of greater complexity $[109,110]$. It uses the electron degrees of freedom as dynamical variables in a set of coupled equations of motion for the nuclei and the electrons. The nuclei have a classical kinetic energy of $M / 2\left[\mathrm{~d} u_{l \kappa} / \mathrm{d} t\right]^{2}$. The trick to the algorithm is that the electrons are given a fictitious kinetic energy of $\mu / 2 \int[\mathrm{d} \psi / \mathrm{d} t]^{2} \mathrm{~d} \vec{r}$, with a mass $\mu$ that is an adjustable parameter (that helps with the stability of the numerical calculations). With this coupling of nuclear and electronic motions, constrained by the orthogonality of the electron wavefunctions, the electrons stay near their ground states during the simulation. The electron wavefunctions do not need to be recalculated at each step of the simulation, offering an improvement in efficiency. The Car-Parrinello algorithm is an adiabatic approximation.

\footnotetext{
28 The notation indicates that the velocity history of each atom is multiplied by the velocity history shifted by $t$, and integrated over all time.
} 
Vibrational Thermodynamics of Materials

\section{Vibrational Entropy of Materials at Lower Temperatures}

\section{Earlier Investigations}

\subsection{POLYMORPHISM}

Polymorphism is the transformation of a material into a new crystal structure by changing $T$ or $P$, without change in composition. The classic texts by J.C. Slater Introduction to Chemical Physics (1939) [111] and by F. Seitz Modern Theory of Solids (1940) [112] have explanations of the role of vibrational entropy in polymorphic phase transformations of pure elements. An early experiment was reported by F. Lange in 1924, who measured the heat capacities of white and gray tin from nearly liquid helium temperature to ambient [113]. The difference in entropy was obtained with the Nernst postulate and by integrating the difference in heat capacity (cf., Eq. 72)

$$
\Delta S_{\mathrm{vib}}^{\alpha-\beta}(T)=\int_{0}^{T} \frac{C_{p}^{\alpha}\left(T^{\prime}\right)-C_{p}^{\beta}\left(T^{\prime}\right)}{T^{\prime}} \mathrm{d} T^{\prime} .
$$

The enthalpy of the transformation was also obtained, and the critical temperature was predicted accurately from the vibrational entropy and the enthalpy [112]. It appears that the entropy of the tin transformation is mostly vibrational. It is big - white tin has a vibrational entropy $0.9 k_{\mathrm{B}}$ /atom greater than gray tin at room temperature. The roles of vibrational entropy in the polymorphism of sulfur, carbon, and iron are discussed semiquantitatively in Seitz's book [112].

Many elemental metals transform from close-packed structures (fcc or hcp) at low temperatures to the bcc structure at high temperatures (and ambient pressure). These include metals with very different electronic structures, such as alkali metals and transition metals. Zener [114] hypothesized that soft transverse phonons, intrinsic to the more open bcc structure, would contribute enough vibrational entropy to favor the bcc structure at high temperatures. Many years later, this idea was subjected to some analysis by Grimvall and Ebbsjö [115], using results from pseudopotential calculations $[116,117]$ and results from force-field calculations of lattice dynamics [118]. Their assessment showed that phonons in bcc crystals were typically a few percent softer than in fcc or hcp, especially for the low transverse modes, supporting Zener's hypothesis. They further argued that this trend could be understood without anharmonic effects, although perhaps not quantitatively. ${ }^{29}$ On the other hand, the fcc and hcp close packed structures seem to have very similar phonon energies, as shown by neutron scattering measurements on Co [119], for example. Likewise, the fcc-based $\mathrm{L}_{2}$ structure of $\mathrm{Co}_{3} \mathrm{~V}$ has a vibrational entropy of only $0.07 k_{\mathrm{B}}$ /atom larger than the ordered hexagonal $\mathrm{hP} 24$ structure of $\mathrm{Co}_{3} \mathrm{~V}$ near

\footnotetext{
29 Their calculations, which assumed a constant volume per atom in both phases [115], are approximate.
} 
the transition temperature of $1320 \mathrm{~K}[120,121]$. On the other hand, for pure ${ }^{57} \mathrm{Fe}$, nuclear resonant inelastic x-ray scattering (NRIXS) measurements of the phonon DOS at elevated pressure and temperature show only a small difference for the bcc $\alpha$-phase and the hcp $\epsilon$-phase [122, 123]. Curiously, another NRIXS study has shown a large difference between the phonon DOS of the hcp $\epsilon$-phase and the fcc $\gamma$-phase [124], contrary to the trend for other metals. The phonon DOS of $\gamma$-Fe was softer than the DOS for $\epsilon$-Fe, giving the fcc $\gamma$-phase the larger vibrational entropy and lower sound velocities.

Electronic entropy also plays an important role in the hcp-bcc transformation [17]. Especially today, ab-initio methods are well suited for comparing vibrational free energies of polymorphic transformations of many elemental metals and compounds (e.g., [125]).

\subsection{Solubilities And Defect Concentrations}

\subsubsection{Diffusion}

Vibrational entropy originates with atom displacements around equilibrium, which are small compared to the atomic displacements of diffusional jumps. When the times between diffusional jumps become very short, however, the distinction between vibrational and configurational degrees of freedom becomes blurred. This is certainly an issue when jump times are comparable to vibrational periods, or even if jump times are comparable to phonon lifetimes. This blurring of vibrational and diffusional phenomena occurs in liquids, for example, where some low-energy modes of vibration become unstable and atom movements occur. Likewise, soft-mode transformations relate vibrational degrees of freedom to structural ones as discussed in Sect. 15.

Even in the common situation where the energetics of the atomic displacements are of order $k_{\mathrm{B}} T \simeq 0.1 \mathrm{eV}$ and the activation barriers for diffusion are of order $1 \mathrm{eV}$, there are relationships between vibrational entropy and diffusional processes. The activation free energy for diffusion, $\Delta G^{*}$, is related to the jump frequency, $\Gamma$, as:

$$
\Gamma(T)=\Gamma_{0} \mathrm{e}^{-\Delta G^{*} / k_{\mathrm{B}} T}=\Gamma_{0} \mathrm{e}^{+\Delta S^{*} / k_{\mathrm{B}}} \mathrm{e}^{-\Delta H^{*} / k_{\mathrm{B}} T},
$$

where $\Gamma_{0}$ is an attempt frequency related to atom vibrations, and $\Delta S^{*}$ is analyzed in terms of vibrations. Zener estimated $\Delta S^{*}$ by how elastic constants soften with temperature [126].

The relationship between atomic vibrations and the entropy of diffusion, $\Delta S^{*}$, was developed formally by G. Vineyard, who assigned the origin of $\Delta S^{*}$ to the vibrational entropy of all harmonic normal modes of the equilibrium solid $\left\{\omega_{i}^{e}\right\}$, and how they change at the saddle point of the diffusional jump $\left\{\omega_{i}^{s}\right\}$ [127]. The expression for the temperature-independent prefactor of Eq. 111 was found to be

$$
\mathrm{e}^{+\Delta S^{*} / k_{\mathrm{B}}}=\frac{\prod_{i=1}^{3 N-1} \omega_{i}^{e}}{\prod_{j=1}^{3 N-1} \omega_{j}^{s}},
$$

where the $3 \mathrm{~N}^{\text {th }}$ frequency of the equilibrium crystal is associated with the attempt frequency of the diffusive jump, $\Gamma_{0}$. Schober, Petry and Trampenau assessed the vibrational entropy and enthalpy of vacancy migration using a lattice Green's function method. For an idealized activation barrier of vacancy migration, they argued 
that the different phonons should be weighted by the phonon DOS with a factor of $1 /(m \omega)^{2}$. This weighting of the phonon DOS accounts for how thermal energy causes larger mean-squared displacements for phonons of lower frequencies [128]. Defect formation entropies of order $2 k_{\mathrm{B}}$ /atom were reported, but these values are not quantitative.

\subsubsection{Solubility}

The thermodynamics of solubility can be understood with the help of Eq. 48, using Eqs. 49, 50, and 52. In the dilute limit of B-atoms in an A-matrix (small c), the free energy is

$$
\begin{aligned}
F(c) & \simeq z N / 2\left[e_{\mathrm{AA}}+2 c\left(e_{\mathrm{AB}}-e_{\mathrm{AA}}\right)\right] \\
& +k_{\mathrm{B}} T N[c \ln c+(1-c) \ln (1-c)] \\
& +k_{\mathrm{B}} T \mathcal{D} N\left[\ln \omega_{\mathrm{AA}}+2 c \ln \left(\omega_{\mathrm{AB}} / \omega_{\mathrm{AA}}\right)\right] .
\end{aligned}
$$

To calculate the solubility, we find the difference in free energy of a dilute A-B alloy with respect to pure $\mathrm{A}$ and pure $\mathrm{B}{ }^{30}$ For illustration, assume all materials have the same crystal structure, and we know that the pure elements have zero configurational entropy of mixing. We calculate $\mathrm{F}(\mathrm{c}=0)$ and $\mathrm{F}(\mathrm{c}=1)$, weight them by their proportions, and take the difference

$$
\begin{aligned}
\Delta F(c)= & F(c)-(1-c) F(0)-c F(1), \\
\Delta F(c)= & -c z N 2 V+c \mathcal{D} N k_{\mathrm{B}} T\left[\ln \left(\frac{\omega_{\mathrm{AB}}^{2}}{\omega_{\mathrm{AA}} \omega_{\mathrm{BB}}}\right)\right] \\
& +N k_{\mathrm{B}} T[c \ln c+(1-c) \ln (1-c)] .
\end{aligned}
$$

Seeking the minimum of $\Delta F(c)$ as $\partial \Delta F(c) / \partial c=0$, the equilibrium concentration for small $c$ is

$$
c=\left(\frac{\omega_{\mathrm{AA}} \omega_{\mathrm{BB}}}{\omega_{\mathrm{AB}}^{2}}\right)^{\mathcal{D}} \exp \left(\frac{z 4 V}{k_{\mathrm{B}} T}\right) .
$$

The exponential in Eq. 116 includes the interchange energy of Eq. 42, which is negative for unmixing systems. The prefactor is $\exp \left(-\Delta S_{\text {vib }} / k_{\mathrm{B}}\right)$, comparable to Eq. 112. The configurational entropy does not appear explicitly in Eq. 116, but the derivation assumed that all sites of the A-crystal are equivalent for placing B-atom solutes.

An analogous expression for a dilute concentration of B-atoms in an A-atom matrix, in equilibrium with a second chemically-ordered phase of precise composition $\mathrm{A}_{1-x} \mathrm{~B}_{x}$, is

$$
\begin{aligned}
& c=\exp \left(-\frac{F(\mathrm{~B})-\frac{1}{x} F\left(\mathrm{~A}_{1-x} \mathrm{~B}_{x}\right)}{k_{\mathrm{B}} T}\right) \\
& c=\exp \left(-\left[S_{\mathrm{vib}}(\mathrm{B})-\frac{1}{x} S_{\mathrm{vib}}\left(\mathrm{A}_{1-x} \mathrm{~B}_{x}\right)\right]\right) \exp \left(-\frac{E(\mathrm{~B})-\frac{1}{x} E\left(\mathrm{~A}_{1-x} \mathrm{~B}_{x}\right)}{k_{\mathrm{B}} T}\right),
\end{aligned}
$$

\footnotetext{
30 The Hume-Rotherey rules, classic arguments for the size of $\{e\}$, and perhaps extendable to $\{\omega\}$, are presented in Section 21.2.
} 
where $E(\mathrm{~B})$ denotes the energy of formation for placing a $\mathrm{B}$-atom on a matrix site and $E\left(\mathrm{~A}_{1-x} \mathrm{~B}_{x}\right)$ denotes the energy of formation per atom of the phase $\mathrm{A}_{1-x} \mathrm{~B}_{x}$ (similarly for $F$ and $S_{\text {vib }}$ ). Any additional sources of entropy, such as electronic entropy, are added to $S_{\text {vib }}$, except for configurational entropy, which was implicitly included when deriving Eqs. 116 and 117.

Equation 118 can be used to obtain vibrational entropies if the energies of formation are known. Because $S_{\text {vib vanishes at low temperatures, experimental approaches }}$ can isolate the $E$ in Eq. 118. More typically, a plot of $\ln c$ versus $1 / T$ can be extrapolated to $1 / T=0$, giving the entropy difference of Eq. 118. Clarence Zener took this approach many years ago, and identified effects of vibrational entropy on solubilities in dilute $\mathrm{Al}$ alloys [129].

Section 1 discussed a key difference between vibrational entropy and configurational entropy that is usually used to calculate solubilities of dilute solutions. The vibrational entropy has an intrinsic temperature dependence, whereas the configurational entropy does not. The relative importance of vibrational entropy therefore increases with temperature, so it is sometimes important to account for a temperature dependence in the difference $S_{\mathrm{vib}}(\mathrm{B})-\frac{1}{x} S_{\mathrm{vib}}\left(\mathrm{A}_{1-x} \mathrm{~B}_{x}\right)$ of Eq. 118. For harmonic solids, this difference would be a constant at high temperatures. Nevertheless, anharmonic effects can be large. It is plausible that changes with temperature of the vibrational entropy of mixing could cause a retrograde solubility. ${ }^{31}$

Huntington, Shirn and Wajda studied the vibrational entropy of formation of lattice defects [130]. They used an Einstein model with a Born-Mayer potential, and considered the changes in vibrational frequencies of individual atoms around a defect, or the frequencies of an interstital atom. These entropies were of order $+1 k_{\mathrm{B}}$ /atom, with the defects giving the larger entropy. Freedman and Nowick [131] built on this work by analyzing data on solubility versus temperature for a set of metallic solid solutions to extract the "excess entropy," (i.e., entropy that was not accounted for by the entropy of mixing, Eq. 11). These contributions were generally positive, meaning that they added to the entropy of Eq. 11, but ranged from $1.1 k_{\mathrm{B}} /$ atom for $\mathrm{Ni}$ in $\mathrm{Ag}$ to $+3.0 k_{\mathrm{B}} /$ atom for $\mathrm{Fe}$ in $\mathrm{Cu}$. Their analysis of vibrational entropy used the Einstein model, but it made the "rather drastic assumption" that there was no overall change in the vibrational frequencies of the solute atoms in the matrix, compared to the averages of the pure elements. This assumption permitted an analysis like that of Zener, which was based on the temperature dependence of the shear modulus. The effects of vibrational entropy seemed to be large.

Desorbo [132] and Oriani [133] used calorimetric methods to estimate the excess entropy of near-equiatomic $\mathrm{Au}-\mathrm{Ni}$, and attributed it to vibrations. Desorbo found the excess entropy to be approximately $+0.3 k_{\mathrm{B}}$ /atom at room temperature, and Oriani states, "The positive value of this deviation and the large magnitude of the vibrational entropy of solution are related to the loosening of the lattice that accompanies formation of the solid solution."

\footnotetext{
31 Of course there is a drastic change in the temperature dependence of this difference at low temperatures below the Debye temperature (cf., Fig. 8b), but such low temperatures are often below the range of validity of $T-c$ phase diagrams.
} 


\subsection{Carbides, Nitrides, and Oxides}

Fernández Guillermet and Grimvall examined thermodynamic data on transition metal carbides, and reported some trends [134]. The standard entropy, primarily vibrational in these ordered structures, and the standard enthalpy were shown to depend on the electron to atom ratio after the carbides were separated into two groups - one with $\mathrm{Sc}$, Ti, V, and the second with $\mathrm{Cr}, \mathrm{Mn}, \mathrm{Fe}, \mathrm{Co}, \mathrm{Ni}$. The first group showed stronger variations in the standard enthalpy and entropy with electron-to-atom ratio than did the second group. For carbides in the first group, standard entropies of formation varied by typically $0.4 k_{\mathrm{B}}$ /atom for different carbon stoichiometries, although the crystal structure also changed. Fernández Guillermet and $\mathrm{Du}$ assessed the thermodynamic properties of the Fe-N system, and noted a large increase in Debye temperature as the nitrogen concentration was increased from the $\mathrm{Fe}_{4} \mathrm{~N}$ to $\mathrm{FeN}_{3}$ (from 300 to $584 \mathrm{~K}$ ) [135]. In the high temperature limit, this corresponds to a large difference in vibrational entropy of $3 k_{\mathrm{B}} \ln (300 / 584) /$ atom $=-2.0 k_{\mathrm{B}}$ /atom for these two compounds.

The pressure-induced phase transformation of $\mathrm{ZnO}$ from the wurtzite structure to the rocksalt structure was the subject of a first-principles computational study using the planewave pseudopotential code VASP, with the code PHONON to calculate phonon frequencies by the direct method [136]. Vibrational entropy was calculated with the harmonic expressions for the phonon frequencies obtained at different volumes (i.e., the quasiharmonic approximation). The transition pressure was found to decrease with temperature (consistent with experimental results), and this was attributed primarily to a larger vibrational entropy of the high-pressure rocksalt structure. The $\mathrm{ZnO}-\mathrm{MgO}$ composition-temperature phase diagram was also calculated, and it was found that vibrational entropy increases the solubility of $\mathrm{ZnO}$ in the rocksalt $\mathrm{MgO}$ by about a factor of two at a given temperature, whereas vibrational entropy decreases the solubility of $\mathrm{MgO}$ in wurtzite $\mathrm{ZnO}$ by a similar factor [136].

Some of the expectations and rules for metal systems may not be transferrable to nitrides or oxides. Structural units in oxide crystals, i.e., metal-oxygen octahedra, can undergo dynamics at low energies by rotating as a rigid unit, whereas the modes involving relative motions of the metal and oxygen atoms typically occur at much higher energies. These "rigid unit modes" of the octahedra [137] can break the symmetry of a structure of packed octahedra, and can be responsible for displacive phase transformations in minerals, for example [138]. In structures where some oxygen atoms at corners of the octahedra are not shared by other octahedra, there is more freedom in these reorientations. The material $\mathrm{ZrW}_{2} \mathrm{O}_{8}$ has such a local structure, and it exhibits an unusual negative thermal expansion at low temperatures. An analogy is opening a pair of scissors, where the total length is reduced as the device is opened, but there is enough open space at the side to accommodate the increase in width. The relevant low energy vibrational modes in $\mathrm{ZrW}_{2} \mathrm{O}_{8}$ have been identified by inelastic neutron scattering [139, 140], and they show a stiffening with increased temperature. A similar negative thermal expansion was observed for the related compound $\mathrm{HfMo}_{2} \mathrm{O}_{8}$, and a similar interpretation was offered in terms of low-frequency optical modes [141]. For quite some time it has been known that RbI exhibits a negative thermal expansion, as does silicon at low temperatures. For 
RbI the mechanical analogy is to a guitar string, where the increased vibrational amplitude of the string pulls inwards its points of suspension [139, 142].

\section{First-Principles Studies of Solutions and Studies of Ordered Compounds}

\subsection{Solubility in Al Alloys}

The interatomic force constants and the phonon DOS of fcc Al metal were obtained by analyzing the thermal diffuse x-ray scattering from a single crystal of Al [143]. ${ }^{32}$ Neutron inelastic scattering experiments were later performed on $\mathrm{Al}$, giving similar results for the lattice dynamics [144, 145]. Many features in the phonon dispersion curves were identified as Kohn anomalies from electron-phonon interactions (Sect. 20). These anomalies are small and difficult to observe, but in principle they could have some thermodynamic effect.

Ab-initio electronic structure calculations have proved valuable for calculating the energy and vibrational entropy of a matrix with solute atoms, and Eq. 118 can be used to find the effects of vibrational entropy on solubility. This approach was used by Ozolins and Asta, who found a large effect of vibrational entropy on the solubility of $\mathrm{Sc}$ in $\mathrm{Al}[146,147]$. Although the solubility of $\mathrm{Sc}$ in $\mathrm{Al}$ is low, calculations without vibrational entropy predict it to be about 27 times lower than has been measured, but the inclusion of vibrational entropy brings good agreement with experient. The Sc dissolved in fcc $\mathrm{Al}$ is in equilibrium with $\mathrm{Sc}$ in the $\mathrm{L}_{2^{-}}$ ordered compound $\mathrm{Al}_{3} \mathrm{Sc}$, so the energies and entropies of ordered compounds were also calculated, as discussed below. The $\mathrm{L}_{2}$ structure of $\mathrm{Al}_{3} \mathrm{Sc}$ proved to be very stiff, with a high bulk modulus and small volume per Sc atom The phonon DOS of the $\mathrm{L}_{2}$ structure had an average frequency higher than either fcc $\mathrm{Al}$ or hcp Sc, giving it a negative entropy of formation. This entropy of formation of $\mathrm{Al}_{3} \mathrm{Sc}$ from fcc $\mathrm{Al}$ and hcp Sc was big, $-0.70 k_{\mathrm{B}} /$ atom. The strong hybridization between Sc $d$ - and $\mathrm{Al} p$-electrons is responsible for short and stiff bonds in this intermetallic compound. For Sc solutes in fcc Al, stiffer first-nearest-neighbor bonds are also expected, and do occur. Nevertheless, the vibrational entropy per Sc atom in fcc $\mathrm{Al}$ is approximately $+0.4 k_{\mathrm{B}} /$ atom, indicating an overall softening of phonon frequencies. The softening occurs for Al-Al bonds in the more distant neighbor shells around the Sc atom. Two inter-related contributions were identified as a source of this softening:

- The element Sc is larger than the $\mathrm{Al}$ atom, and tends to increase the lattice parameter of the alloy. The elongation of the Al-Al bonds is a source of softening.

- $\mathrm{Al}$ is more electronegative than Sc, so we expect a charge transfer and a charge perturbation around $\mathrm{Sc}$ atoms. The conduction electrons of $\mathrm{Al}$ redistribute around the charge perturbation, much as Friedel oscillations in a free electron gas. The entropic effects of the charge disturbances are of similar size as the effects of volume expansion.

\footnotetext{
32 This was sophisticated work, especially in its day, and few other phonon studies have since been done this way.
} 
Ozolins, Sadigh, and Asta [148] performed a computational study on the solubility of $\mathrm{Si}$ in Al. The Al-Si phase diagram is a simple eutectic, so the relevant phases are fcc Al, fcc Al-Si, and dc Si. The phonons in dc Si are quite stiff in comparison to the other phases. Removing a $\mathrm{Si}$ atom from covalently-bonded dc $\mathrm{Si}$ and placing it in fcc $\mathrm{Al}$ has the net effect of lowering the vibrational frequencies of all atoms. The vibrational frequencies at $\mathrm{Al}$ atoms in the vicinity of Si solutes are not altered significantly. (There is no large charge redistribution as for the case of Sc, for example.) With the dominant effect of solubility being the loss of short, stiff covalent bonds in $\mathrm{dc} \mathrm{Si}$, the vibrational entropy of formation for dilute $\mathrm{Si}$ in $\mathrm{Al}$ was calculated as being $+2.6 k_{\mathrm{B}} / \mathrm{Si}$ atom, quite a large effect. This seems qualitatively consistent with the softening of transverse acoustic modes measured by inelastic neutron scattering on supersaturated Al-Si solid solutions [149]. It should be noted that Ozolins, et al., encountered difficulties with the LDA approximation in this study of Si solubility, owing to differences in the exchange-correlation energy caused by charge inhomogeneities in the different phases.

\subsection{Aluminum Compounds}

Aluminum alloys are of high technological importance because pure $\mathrm{Al}$ is mechanically soft, and second phases can provide strengthening mechanisms. One of the best known examples of precipitation hardening occurs for $\mathrm{Al}-\mathrm{Cu}$ with the nonequilibrium phases $\theta^{\prime \prime}$ and $\theta^{\prime}$. These eventually transform to the equilibrium $\theta$ phase, so the free energy of the $\theta$ phase must be lower than that of the metastable $\theta^{\prime}$ phase. Nevertheless, Wolverton and Ozolins calculated the electronic energy of the $\mathrm{Al}_{2} \mathrm{Cu}$ $\theta$ phase, and found it to be $15 \mathrm{meV} /$ atom larger than for the $\theta^{\prime}$ phase [150]. The stability of the $\theta$ phase was attributed to its vibrational entropy, approximately $+0.37 k_{\mathrm{B}}$ /atom larger than that of $\theta^{\prime}$ in the harmonic approximation. An entropic stabilization of the $\theta$ phase implies a phase transformation to the lower-energy $\theta^{\prime}$ phase upon cooling. The transformation temperature is predicted to occur at $T_{c}=15 \mathrm{meV} / 0.37 k_{\mathrm{B}} \simeq 200^{\circ} \mathrm{C}$. There is weak experimental evidence for this phase transformation, but it should be quite sluggish at this low temperature.

The entropic stabilization of the $\theta$ phase may originate with features of its lowsymmetry structure. The lowest-frequency optical modes in the $\theta$ phase allow for perpendicular motions of neighboring $\mathrm{Al}$ and $\mathrm{Cu}$ atoms, with minimal change in $\mathrm{Al}-\mathrm{Cu}$ bond lengths. Wolverton and Ozolins suggest that vibrational entropy stabilization may be important for other low-symmetry structures such as Laves phases in transition metal systems. The general idea is that the low symmetry may result in a less favorable bonding energy, but this is compensated at higher temperatures by a larger vibrational entropy originating from floppy atom motions.

Vibrational entropies of formation were calculated for several intermetallic compounds in the Al-Sc system by Asta and Ozolins [147]. Although the vibrational entropy of formation of the stiff $\mathrm{L}_{2} \mathrm{Al}_{3} \mathrm{Sc}$ from fcc $\mathrm{Al}$ and hcp Sc had the largest magnitude, $-0.77 k_{\mathrm{B}}$ /atom, the B2 AlSc and orthorhombic AlSc compounds had vibrational entropies of formation of $-0.58 k_{\mathrm{B}}$ /atom and $-0.24 k_{\mathrm{B}}$ /atom. The orthorhombic $\mathrm{B}_{f}$ compound ( $\mathrm{BCr}$ prototype) has a lower symmetry than the cubic $\mathrm{L} 1_{2}$ and $\mathrm{B} 2$ structures, and its low-frequency optical modes give it the least negative vibrational entropy of formation of these compounds with strong Al-Sc bonds. 


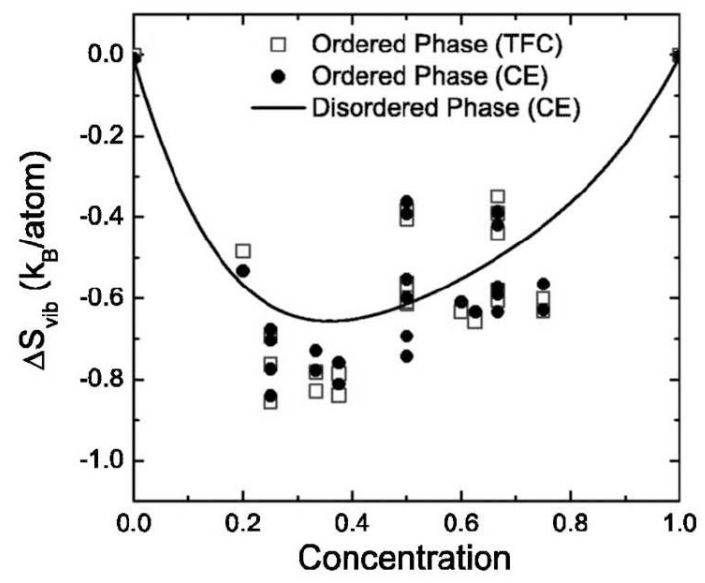

Figure 12. Vibrational entropies of mixing of $\mathrm{Hf}$ in $\mathrm{Al}$. The symbols are for ordered structures. Open squares were calculated by the transferable force constant approach while the solid circles are the values predicted by the cluster expansion method The solid lines are predictions from the cluster expansion method for a disordered fcc solid solution of Al-Hf. After [45].

Quasicrystals, or icosahedral-phase compounds, are found in a number of aluminumtransition metal systems as metastable phases, and are in fact stable phases in some systems such as $\mathrm{Al}-\mathrm{Co}-\mathrm{Ni}$. In the case of $\mathrm{AlCo}_{x}$, where $0.23<x<0.26$, a large number of crystal structures have been reported, some of which are quasicrystal approximants. It is challenging to determine which of these is the thermodynamicallystable structure. Energetic comparisons with ab-initio calculations can help. In such work by Mihalkovic and Widom [151], the orthorhombic and monoclinic $\mathrm{Al}_{13} \mathrm{Co}_{4}$ structures were found to be unstable energetically. Nevertheless, a combination of defect entropy and vibrational entropy made these $\mathrm{Al}_{13} \mathrm{Co}_{4}$ structures stable at temperatures above $750 \mathrm{~K}$.

As discussed in Sect. 5.3, Liu, et al., identified chemical modifications that improve the agreement between the bond-stiffness-versus-bond-length model and first principles calculations for Al-TM alloys, where $\mathrm{TM}=\{\mathrm{Ti}, \mathrm{Zr}, \mathrm{Hf}\}$ [45]. It is possible that these concentration dependences are correlated to the difference in electronegativity between the $\mathrm{TM}$ and the $\mathrm{Al}$ atom - this difference is smallest for $\mathrm{Al}-\mathrm{Ti}$, as are the concentration dependences of the curves of bond stiffness versus bond length. Consider, for example, the systematics of Al-Hf shown in Fig. 12. The strong hybridization of $\mathrm{Al} p$ - and $\mathrm{Hf} d$-electrons tends to build up electron density in the bonding levels between Al-Hf $1 \mathrm{NN}$ pairs, causing a stiffening of phonons and a negative entropy of mixing. Figure 12 shows that the entropies of mixing are large and negative in all cases, typically $-1 k_{\mathrm{B}}$ /atom. The systematics of ordering are less obvious. The ordered phases tend to be stiffer, with negative vibrational entropies of ordering of approximately $-0.2 k_{\mathrm{B}} /$ atom, but there are exceptions, perhaps associated with lower symmetry structures.

\subsection{Low-Symmetry Phases at High Temperatures}

Any survey of alloy phase diagrams shows examples of chemically-ordered compounds that exist at high temperatures, but decompose to other phases at lower 
temperature. High-temperature phases are stabilized by entropy, but there is no configurational entropy for chemically-ordered structures, of course, and vibrational entropy likely plays a role in their high-temperature stability. ${ }^{33}$ Many of these hightemperature ordered phases are low-symmetry crystal structures. The B $8_{1} \mathrm{NiAs}$ structure is such an example, and may sometimes be stabilized by vibrational entropy. The B 81 structure has a number of asymmetrical intersticies that could accommodate large-amplitude atom vibrations, which may be of low frequency and anharmonic. More interestingly, in about half the phase diagrams where the $\mathrm{B} 8_{1}$ structure appears, it is not stable at low temperatures, but only appears at high temperature. Vibrational entropy seems a likely source of stabilizing the B $8_{1}$ structure in $\mathrm{AlCu}, \mathrm{CrSe}, \mathrm{CuSn}, \mathrm{FeS}, \mathrm{CuIn}, \mathrm{InPt}, \mathrm{PdSb}, \mathrm{FeSn}, \mathrm{CrTe}, \mathrm{FeTe}$, NiS, CrS, CoSe and FeSe, which appear only at high temperatures [152, 153]. Many of these compounds exist off the equiatomic stoichiometry, however, suggesting there is also a role for defect entropy in their high-temperature stability.

At high temperatures, the full thermodynamics is likely more complex. For example, the $\mathrm{B} 8_{1}$ NiAs structure of CuSn undergoes an ordering transformation to a longperiod structure at temperatures below $187^{\circ} \mathrm{C}$, with an ordering enthalpy measured to be $-0.26 \pm 0.03 \mathrm{~kJ} /$ mole [154], implying an ordering entropy of $-0.07 k_{\mathrm{B}}$ /atom. This is an unusually small entropy for chemical ordering, suggesting that the lowtemperature ordered phase has a relatively large vibrational entropy (or only a small fraction of atoms are involved in the ordering process, implying that the chemical disorder at high temperatures is small). The $\mathrm{B} 8_{1}$ phase of FeSe is a case where a number of other sources of entropy may affect its stability. ${ }^{34}$ It is an antiferromagnet with a Néel temperature of $600 \mathrm{~K}$, which is very close to the temperature where the $\mathrm{B} 8_{1}$ phase appears on the phase diagram $(633 \mathrm{~K})$. Furthermore, the Fermi level of $\mathrm{B} 8_{1}$ FeSe lies in a deep valley between two closely-spaced, high peaks in the electronic DOS [157]. Thermal smearing, discussed below in Section 20, should increase the DOS at the Fermi level, enhancing the electron-phonon coupling, although this trend should reverse at higher temperatures. Both magnetism and the adiabatic electronphonon interaction should be considered when assessing the stability of the $\mathrm{B} 8_{1}$ phase of FeSe.

As a curious counterexample, a high-symmetry B2-ordered phase exists in the $\mathrm{Cu}-\mathrm{Zr}$ system only above $715^{\circ} \mathrm{C}$ at $51.5 \% \mathrm{Zr}$ [152]. This $\mathrm{B} 2$ phase undergoes a eutectoid transformation below $715^{\circ} \mathrm{C}$ to two other intermetallic compounds of lower symmetry $[158,159]$. Thermodynamic assessments give it an entropy of stabilization of $0.46 k_{\mathrm{B}}$ /atom with respect to pure $\mathrm{Cu}$ and $\mathrm{Zr}$ [160], which seems too large to be accounted for by configurational entropy (unless there is a high degree of chemical disorder in the B2 structure). Although it seems reasonable to propose a rule of thumb that vibrational entropy helps stabilize low-symmetry structures at high temperatures, the $\mathrm{B} 2$ phase in $\mathrm{Cu}-\mathrm{Zr}$ serves as a warning. Another example is the hexagonal C32 structure. It is not a low-symmetry structure, but many C32 diboride

\footnotetext{
33 Some partial chemical disorder may contribute configurational entropy, and antisite defects may also influence the phonon dynamics in ways that are not understood.

34 It has recently been found that the B10 structure (PbO prototype) of Fe-Se, stable at more Fe-rich compositions, is a superconductor with similarities to the new iron arsenates [155, 156]. Perhaps other charge or spin excitations in the B8 1 phase of Fe-Se are relevant for phase stability.
} 
phases are stable to extremely high temperatures. Some, like $\mathrm{MnB}_{2}$, are unstable at lower temperatures.

\section{Chemical Order-Disorder Transformations}

\subsection{Transition Metal Aluminides}

\subsection{1. $\mathrm{Fe}_{3} \mathrm{Al}$}

When the atom displacements, $u$, are comparable to the inverse of the scattering wavevector, $Q$, an elastic Bragg diffraction is suppressed by the Debye-Waller factor $\exp \left(-Q^{2}\left\langle u^{2}\right\rangle\right)$, as discussed in Sect. 8.1. A closely-related effect occurs with the local probe methods "extended x-ray absorption fine structure" (EXAFS) or "extended electron energy-loss fine structure" (EXELFS), which measure scattering above a core ionization edge of an atom. The electron leaving the ionized atom has a wavelength that allows self-interference by backscattering from the nearest-neighbor shells of atoms. For energies $E$ above the absorption edge $E_{\mathrm{a}}$, the wavevector, $k$, of the outgoing electron is $E-E_{\mathrm{a}}=\hbar^{2} k^{2}\left(2 m_{\mathrm{e}}\right)^{-1}$. When $2 \pi / k$ matches the roundtrip nearest-neighbor distance, constructive interference occurs, and the ionization probability is slightly enhanced. ${ }^{35}$ The Fourier transform of the EXAFS or EXELFS oscillations above $E_{\mathrm{a}}$ can be used to identify nearest-neighbor distances and the scattering strengths of the neighboring atoms. If the nearest-neighbor atoms are vibrating with respect to the ionized atom, the self-interference of the outgoing electron is suppressed. The effect is essentially the same as a Debye-Waller factor, but it is associated with the mean-squared relative displacement (MSRD) between neighboring atoms. Measurements of the MSRD as functions of temperature for Fe and $\mathrm{Al}$ atoms in $\mathrm{DO}_{3}$-ordered and disordered solid solutions of $\mathrm{Fe}_{3} \mathrm{Al}$ are shown in Fig. 13 from work in 1990 [161]. What is important is that the MSRD of Al atoms is larger, and more temperature-dependent, in the disordered than in the ordered phase. A similar but weaker trend is found for Fe atoms. Fitting these data to MSRD curves calculated with a local Einstein model provides characteristic temperatures listed in the caption. With these Einstein temperatures we can estimate a difference in vibrational entropy of $0.4 \mathrm{k}_{\mathrm{B}}$ /atom, with the disordered material having the larger entropy. These EXELFS measurements were repeated with better experimental technique, but with similar results [162].

Subsequent calorimetry work using Eq. 110 [163] showed that the difference in vibrational entropy of disordered and ordered $\mathrm{Fe}_{3} \mathrm{Al}$ is $(0.1 \pm 0.03) \mathrm{k}_{\mathrm{B}} /$ atom, much smaller than the $0.4 \mathrm{k}_{\mathrm{B}}$ /atom deduced from the simple EXELFS interpretation with an Einstein model. The discrepancy gives insight into the origin of the change in vibrational entropy. The EXELFS method is more sensitive to high-frequency longitudinal phonons than other phonons [164]. If disordering affects primarily the high-frequency phonons, EXELFS will tend to overestimate the change in vibrational entropy.

Figure 14 presents results on ${ }^{57} \mathrm{Fe}_{3} \mathrm{Al}$ from nuclear resonant inelastic x-ray scattering (NRIXS) measurements, and partial DOS curves calculated for the $\mathrm{DO}_{3}$-ordered

\footnotetext{
${ }^{35}$ There are numerous other factors to consider, especially the change in phase of the electron wavefunction upon scattering, but these are beyond the scope of the present article.
} 
(a)

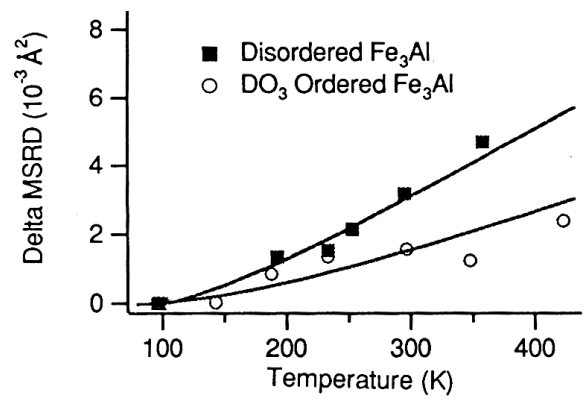

(b)

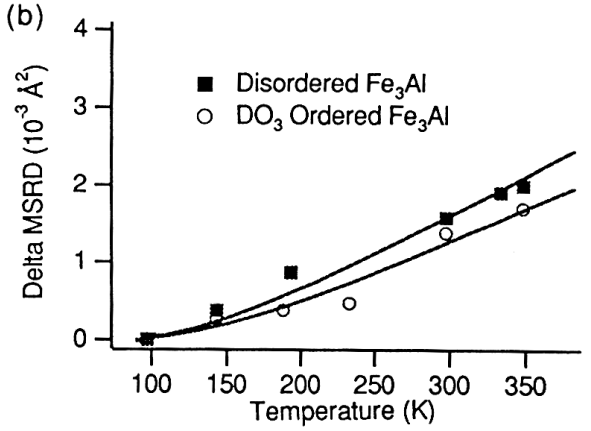

Figure 13. (a) Experimental MSRD between $\mathrm{Al}$ atoms and their $1 \mathrm{nn}$ shell relative to data at $96 \mathrm{~K}$. Lines are calculations of MSRD based on Einstein temperatures of $350 \mathrm{~K}$ for $\mathrm{Al}$ in disordered $\mathrm{Fe}_{3} \mathrm{Al}$, and $460 \mathrm{~K}$ for $\mathrm{Al}$ in $\mathrm{DO}_{3}$-ordered $\mathrm{Fe}_{3} \mathrm{Al}$. (b) Experimental MSRD between Fe atoms and their 1nn shell relative to data at $96 \mathrm{~K}$. Lines are calculations of MSRD based on Einstein temperatures of $400 \mathrm{~K}$ for $\mathrm{Fe}$ in disordered $\mathrm{Fe}_{3} \mathrm{Al}$, and $440 \mathrm{~K}$ for $\mathrm{Fe}$ in $\mathrm{DO}_{3}$-ordered $\mathrm{Fe}_{3} \mathrm{Al}$.

structure [165]. The NRIXS method is similar to incoherent inelastic neutron scattering, but the spectral intensities of NRIXS are weighted entirely on the ${ }^{57} \mathrm{Fe}$ atoms, which exhibit the nuclear resonance. The calculated partial phonon DOS of Al shows that the optical modes at energies around $43 \mathrm{meV}$ are dominated by the motions of the $\mathrm{Al}$ atoms. The shapes of the $\mathrm{Fe}$ and $\mathrm{Al}$ partial DOS curves at the energies of the optical modes are rather similar. ${ }^{36}$ The $8[\mathrm{c}]$ Fe atoms, which are first neighbors of the $\mathrm{Al}$ atoms, have small displacements in response to the $\mathrm{Al}$ vibrations. Nevertheless, the partial DOS of ${ }^{57} \mathrm{Fe}$ is measured reliably owing to the low background and high sensitivity of the NRIXS method. Figure 14 shows that with disordering, the spectral contribution from the optical modes becomes less distinct, but more importantly these modes shift to lower energy. This effect depends primarily on the short-range order in the alloy, and substantial changes to the phonons occur well before any superlattice diffractions from long-range order are observed in diffraction measurements [166]. A downwards shift of about $10 \%$ for a quarter of the modes accounts for a change in vibrational entropy of $-0.25 \times 3 \ln (0.9)=0.08 k_{\mathrm{B}} /$ atom, in good agreement with the calorimetry result of $(0.1 \pm 0.03) \mathrm{k}_{\mathrm{B}} /$ atom. It is unfortunate that the partial phonon DOS of Al cannot be measured, but the MSRD data of Fig. 13a also point to changes in the $\mathrm{Al}$ atom dynamics at high frequency as a main source of the vibrational entropy of the order-disorder transformation in $\mathrm{Fe}_{3} \mathrm{Al}$.

\subsection{2. $\mathrm{Ni}_{3} \mathrm{Al}$}

Section 4.2 discussed how phonon frequencies are expected to increase with the strengths of chemical bonds, or pairwise interaction energies. For an alloy with strong ordering tendencies (a large $V$ and a high critical temperature (Eqs. 62, 64, 70)), we might expect a large change in vibrational entropy upon disordering. The critical temperature for $\mathrm{D}_{3}$ ordering in $\mathrm{Fe}_{3} \mathrm{Al}$ is $820 \mathrm{~K}$, although $\mathrm{B} 2$ order persists to $1100 \mathrm{~K}$ or so. On the other hand, for $\mathrm{Ni}_{3} \mathrm{Al}$ the $\mathrm{L}_{2}$ ordered structure persists to melting at $1635 \mathrm{~K}$, and chemical disorder is notoriously difficult to induce in $\mathrm{Ni}_{3} \mathrm{Al}$.

\footnotetext{
${ }^{36}$ Three phonon branches out of twelve (4-atom unit cell) correspond to these optical modes, so from $40-45 \mathrm{meV}$ the partial DOS of Al must sum with the Fe partial DOS to account for $25 \%$ of the total DOS.
} 


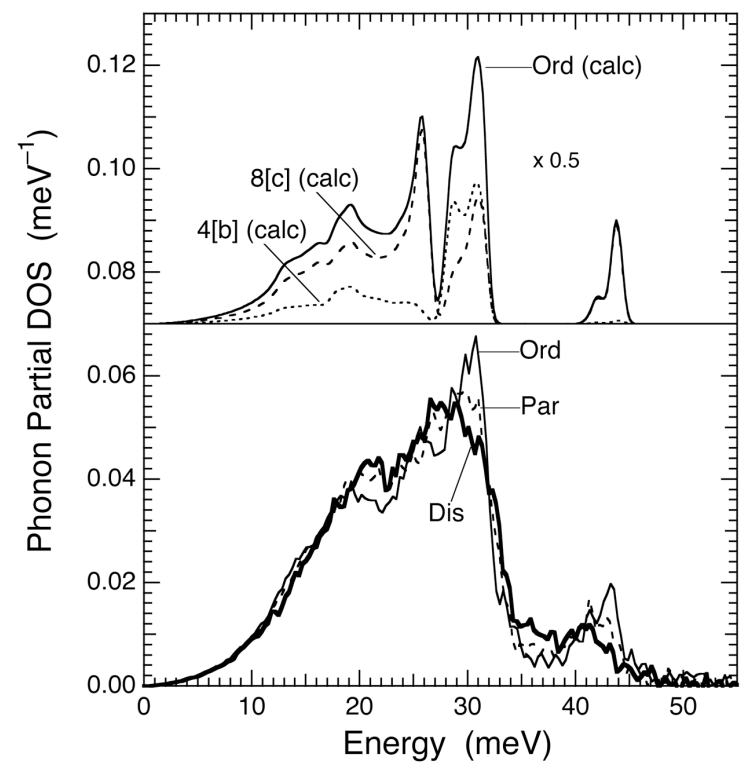

Figure 14. Phonon partial DOS curves for ${ }^{57} \mathrm{Fe}$ in ${ }^{57} \mathrm{Fe}_{3} \mathrm{Al}$. Top: Calculated with a Born - von Kármán model using force constants from prior neutron scattering measurements on single crystals of $\mathrm{DO}_{3}$-ordered $\mathrm{Fe}_{3} \mathrm{Al}$, convoluted with an experimental resolution function. Bottom: ${ }^{57} \mathrm{Fe}$ phonon partial DOS curves from $\mathrm{Fe}_{3} \mathrm{Al}$ with high long-range order ("Ord"), some short-range order ("Par"), and no measurable short-range order ("Dis").

Disordered samples of $\mathrm{Ni}-25 \mathrm{at} . \% \mathrm{Al}$ were prepared by physical vapor deposition onto cold substrates, and their heat capacity was compared to annealed samples with $\mathrm{L} 1_{2}$ chemical order using differential scanning calorimetry (Sect. 7) and Eq. 110 [167, 168]. X-ray Debye-Waller factor measurements and EXELFS MSRD measurements were also performed on these pairs of ordered and disordered samples, indicating that the vibrational entropy of the disordered $\mathrm{Ni}_{3} \mathrm{Al}$ was $0.3 k_{\mathrm{B}}$ /atom larger than the ordered (error bars are probably \pm 0.1 ).

Inelastic neutron scattering measurements were performed on disordered $\mathrm{Ni}_{3} \mathrm{Al}$ prepared by high-energy ball milling, and experimental spectra are presented in Fig. 15a [169]. The optical modes at $39 \mathrm{meV}$ in the ordered alloy are clearly suppressed by disordering, and the disordered spectra show new intensity around $32 \mathrm{meV}$. The phonon DOS of the ordered $\mathrm{Ni}_{3} \mathrm{Al}$ was possible to deduce from the experimental spectra by a neutron weight correction based on a comparison with a Born-von Kármán calculation from prior work on single crystals of $\mathrm{L}_{2} \mathrm{Ni}_{3} \mathrm{Al}$ [170]. As expected by footnote 36 for ordered $\mathrm{Fe}_{3} \mathrm{Al}$, there is indeed about $25 \%$ of the intensity in the optical mode peak around $39 \mathrm{meV}$ in the neutron-weight corrected spectrum in Fig. 15b. The proper correction for the disordered sample is less obvious, but it should lie between two extremes. The first, labeled "Dis 1" in Fig. 15b, assumes a virtual crystal, with no neutron weighting of the different parts of the phonon DOS. The intensity at high energies is probably too low, since many $\mathrm{Al}$ atoms are still expected to vibrate in local modes. The second, labeled "Dis 2" in Fig. 15b, used the same neutron weight correction as for the ordered alloy. This method probably gives too much intensity in the modes around $39 \mathrm{meV}$ because with disorder there is clearly 

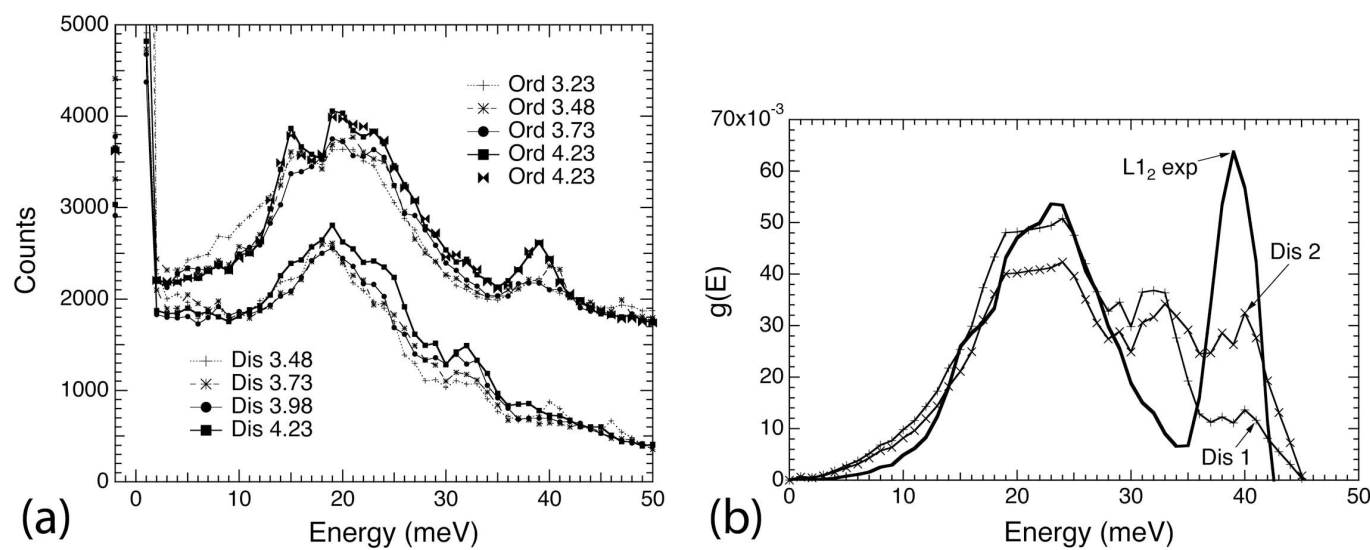

Figure 15. (a) Neutron energy loss spectra for ordered and disordered $\mathrm{Ni}_{3} \mathrm{Al}$ powders, obtained for four values of $Q$. Data for the ordered alloy are offset by 1500 counts. (b) Phonon DOS curves of ordered $\left(\mathrm{L}_{2}\right)$ and disordered $\mathrm{Ni}_{3} \mathrm{Al}$ (Dis1, Dis 2). Neutron weight corrections were performed for the ordered data. There is uncertainty in the neutron weight correction for the disordered alloy, with two extremes shown as Dis 1 and Dis 2 .

some intensity that shifted to lower energies, which are suppressed somewhat by the renormalization of the DOS curve to unit area. The effects of disorder on the optical modes of $\mathrm{Ni}_{3} \mathrm{Al}$, which are dominated by the motions of $\mathrm{Al}$ atoms, are larger than the similar effects found for $\mathrm{Fe}_{3} \mathrm{Al}$, consistent with the calorimetric measurements of a vibrational entropy of ordering that are a factor of 2 or 3 larger for $\mathrm{Ni}_{3} \mathrm{Al}$ than $\mathrm{Fe}_{3} \mathrm{Al}$.

After the first report that vibrational entropy was important to the thermodynamics of ordering in $\mathrm{Ni}_{3} \mathrm{Al}$ [167], a number of computational studies addressed the entropy difference of ordered and disordered $\mathrm{Ni}_{3} \mathrm{Al}$ [171, 172, 173, 174, 175, 176]. Agreement with experiment, or with each other, was not good. A density functional theory study on $\mathrm{Ni}_{3} \mathrm{Al}$ by van de Walle, Ceder and Waghmare [176], arguably the most sophisticated at its time, found essentially no difference of vibrational entropy between the ordered and disordered materials. The biggest effects were in the optical modes involving $\mathrm{Al}$ atom motions, as was found experimentally, but in the DFT calculations these modes were shifted to higher energies in the disordered material. One source of this discrepancy is the difference in lattice parameter of the ordered and disordered $\mathrm{Ni}_{3} \mathrm{Al}$. Phonon frequencies are highly sensitive to lattice parameter, as discussed in Sect. 5.2. Experimentally, the lattice parameter of the ordered material was larger than the disordered, but the DFT calculations gave the opposite result, and EAM calculations gave an even larger difference [172], again in the opposite direction as experiment. With Eq. 68 we obtain $\delta S=3 \ln \left(1+\gamma \delta V / V_{0}\right)=0.17 k_{\mathrm{B}} /$ atom, using $\delta V / V_{0}=0.03$ for the difference between ordered and disordered material prepared by high-energy ball milling, and assuming $\gamma=2$. Unfortunately, this worsens the agreement between theory and experiment, so a more plausible reason is that the chemical disorder in ball milled $\mathrm{Ni}_{3} \mathrm{Al}$ is not of the antisite variety assumed in the computational work. This makes it difficult to do a rigorous comparison between theory and experiment. Another peculiarity of the experimental phonon DOS of the disordered material in Fig. 15b is the enhanced intensity at energies below 15 
$\mathrm{meV}$. This is an interesting aspect of nanostructured materials, discussed below in Section 16.2. The positions of the Van Hove singularities in the phonon DOS of nanostructured materials are usually the same as for bulk materials, however, so the effects on optical modes, other than broadening, are not expected to be large.

\subsection{B2 Ordering in CuZn and FeCo}

In 1938, a few years after Bragg and Williams [177] and Bethe [178] explained orderdisorder transformations without vibrational entropy, Nix and Shockley argued that the vibrational entropy change of order-disorder transformations could be assessed from Debye temperatures [179]. Zener assessed the temperature-dependence of the phase boundaries of disordered $\alpha$ - and $\beta$-phase $\mathrm{Cu}-\mathrm{Zn}$, and argued that the $\beta$ phase must be stabilized in part by vibrational entropy (which works out to be approximately $0.13 k_{\mathrm{B}} /$ atom) [180]. The role of vibrational entropy in the chemical order-disorder transformation of $\beta$-brass was considered with increasing sophistication in the mid-twentieth century. In 1953 Saroléa used a Lennard-Jones model and a method of physical chemistry to deduce that vibrational entropy would cause a small 4.5\% decrease in the ordering transition temperature in CuZn [181]. In 1955, Booth and Rowlinson developed a classical partition function for vibrations in the Einstein model to extend the Bragg-Williams model of ordering. They estimated a $50 \%$ increase in the heat capacity of $\beta$-brass across the order-disorder transition, and a lowering of the critical temperature [182]. Using a specialized calculation, Wojtowicz and Kirkwood found that the decrease in critical temperature was about a factor of two [183]. The B2 ordering transformation in $\mathrm{Cu}-\mathrm{Zn}$ seems worthy of study by more modern methods.

The alloy Fe-50at.\%Co has a B2 order-disorder transformation at $1000 \mathrm{~K}$. The enthalpy of the ordering transformation, $\Delta H_{\text {ord }}$, was measured by differential scanning calorimetry, and found to be $4.4 \mathrm{~kJ} / \mathrm{mol}[184,185]$ giving a transformation entropy $\Delta S_{\text {ord }}=\Delta H_{\text {ord }} / T_{\text {ord }}=0.53 k_{\mathrm{B}} /$ atom. This could be accounted for by the configurational entropy alone, but other sources should be assessed. Both the disordered and ordered phases are ferromagnetic at $T_{\text {ord }}$. It seems that the magnetic entropy does not change significantly with ordering, since the saturation magnetization has little change around $1000 \mathrm{~K}$ [186], and in-situ Mössbauer spectrometry studies show that the ordering process at $800 \mathrm{~K}$ does not cause any discontinuity in the temperature dependence of the hyperfine magnetic field [187]. Although no phonon measurements have been performed at elevated temperatures, phonon DOS curves of ordered and disordered Fe-50at.\%Co alloys have been measured at $300 \mathrm{~K}$ [188]. The features of the phonon DOS curves are somewhat sharper in the B2-ordered sample, but there is no net shift of the phonon frequencies that could account for a significant difference in vibrational entropy between the ordered and disordered materials. Interestingly, this is qualitatively consistent with the prediction of Eq. 70 - unlike the general trend for ordered compounds, the B2 ordered phase of FeCo has a lattice parameter that is $0.2 \%$ larger than for the bcc solid solution [189]. With Eq. 69 this dilation predicts a small entropic contribution of perhaps $0.03-0.04 k_{\mathrm{B}}$ /atom. The role of vibrational entropy on the $\mathrm{B} 2$ ordering transformation in FeCo appears to be small. 


\section{3. $\mathrm{L}_{2}$ Ordering}

\subsection{1. $C u_{3} A u$}

In 1954, Bowen reported measurements of the electrical resistivity of ordered and disordered $\mathrm{Cu}_{3} \mathrm{Au}$ over a range of cryogenic temperatures [190]. Debye temperatures of 175 and $197 \mathrm{~K}$ were reported for the disordered and ordered alloys, suggesting that the disordered phase had a larger vibrational entropy by the amount ${ }^{37}$ $3 \ln (197 / 175)=0.35\left[k_{\mathrm{B}} /\right.$ atom]. Flinn, McManus and Rayne [191] measured the elastic constants of ordered and disordered $\mathrm{Cu}_{3} \mathrm{Au}$ from 4.2 to $300 \mathrm{~K}$, finding differences of less than 1\%. They criticized Bowen's results, "It is concluded that little significance can be attached to [Debye temperatures] obtained from resistance measurements on $\mathrm{Cu}_{3} \mathrm{Au} . " 38$ Rayne had performed an earlier calorimetric study on ordered and disordered $\mathrm{Cu}_{3} \mathrm{Au}$ [193], and found evidence that vibrational entropy of ordering was between the values reported in [190] and [191]. Nevertheless, the message from Flinn, McManus and Rayne was that the difference in vibrational entropy of ordered and disordered $\mathrm{Cu}_{3} \mathrm{Au}$ was not important thermodynamically. This thinking persisted for decades.

Differences in the phonon spectra of ordered and disordered $\mathrm{Cu}_{3} \mathrm{Au}$ were measured more recently [194] with a triple-axis spectrometer, but the thermodynamic consequences were not assessed. There is risk, however, in interpreting the peaks in the phonon scans at constant $\vec{Q}$, measured on single crystals of disordered material. Specifically, the peaks in $I(\varepsilon)$ from the disordered solid solution of $\mathrm{Cu}_{3} \mathrm{Au}$ were interpreted as energies of phonons in a "virtual" fcc crystal, which assumes one species of atom with average properties of $\mathrm{Cu}$ and $\mathrm{Au}$ atoms. The virtual crystal is not expected to be trustworthy when the $\mathrm{Cu}$ and $\mathrm{Au}$ atoms differ in mass by a factor of three, and differ considerably in their atomic radius. Nevertheless, this approach was used in an earlier reconciliation of the phonon dispersion curves with heat capacity data $[168,195]$. The calorimetric measurements by Nagel, et al., showed that as a disordered solid solution, $\mathrm{Cu}_{3} \mathrm{Au}$ has a larger vibrational entropy than for the $\mathrm{L}_{2}$ structure by about $0.14 \pm 0.05 k_{\mathrm{B}}$ /atom $[195,196]$. A similar value was obtained from a lattice dynamics calculation with forces derived from tight-binding potentials with a virtual crystal approximation [197] and other potentials [174, 198], although there were differences in interpretation. Surface effects were also investigated [199].

The neutron weighting problem (Sect. 8.3) is a major challenge for phonon spectra of $\mathrm{Cu}_{3} \mathrm{Au}$ measured by time-of-flight instruments. An atom of $\mathrm{Cu}$ is 3.2 times more efficient than $\mathrm{Au}$ for phonon scattering. Because the atom masses differ by a factor of 3.1, we expect a tendency of $\mathrm{Au}$ atoms to have larger displacements in lowenergy modes, and $\mathrm{Cu}$ atom motions to dominate at higher energies. Correcting for neutron weighting is not difficult when a Born-von Kármán model is developed for ordered phases with a periodic unit cell, but it is difficult to perform for disordered solid solutions. It is probably more reliable to work with the entropy as an integral quantity of the phonon DOS, defining a neutron-weighted vibrational entropy, $S_{\text {vib }}^{\text {nw }}$,

\footnotetext{
37 The factor of 3 arises because each atom has 3 degrees of freedom in 3 dimensions.

38 Rossiter and Bykovec later showed that the electrical resitivity of $\mathrm{Cu}_{3} \mathrm{Au}$ depends in a complex way on the presence of antiphase domain boundaries, and is not a simple function of order parameter [192].
} 


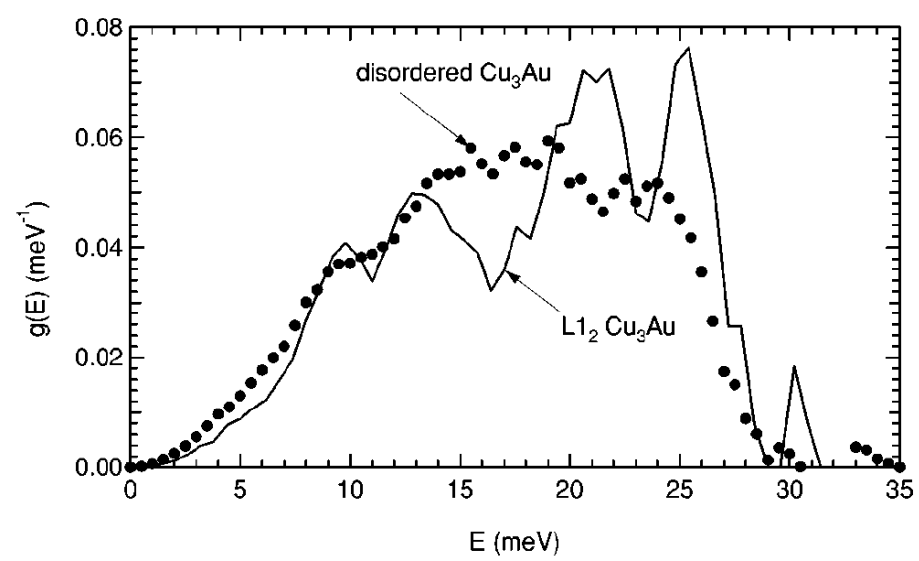

Figure 16. Phonon DOS curves of ordered and disordered $\mathrm{Cu}_{3} \mathrm{Au}$, corrected for neutron weighting [201].

that differs from the true vibrational entropy $S_{\mathrm{vib}}$ :

$$
\begin{aligned}
S_{\mathrm{vib}}^{\mathrm{nw}} & =\frac{3.2(1-x) S_{\mathrm{vib}}^{\mathrm{Cu}}(x)+x S_{\mathrm{vib}}^{\mathrm{Au}}(x)}{3.2(1-x)+x}, \\
S_{\mathrm{vib}} & =(1-x) S_{\mathrm{vib}}^{\mathrm{Cu}}(x)+x S_{\mathrm{vib}}^{\mathrm{Au}}(x)
\end{aligned}
$$

where $S_{\mathrm{vib}}^{\mathrm{Cu}}(x)$ and $S_{\mathrm{vib}}^{\mathrm{Au}}$ are the phonon partial DOS functions for $\mathrm{Cu}$ and $\mathrm{Au}$ at the Au concentration, $x .{ }^{39}$ Several approaches were used to correct Eq. 119 for the neutron weighting of $\mathrm{Cu}$-Au alloys [200], with the best estimate that the disordered phase has a larger vibrational entropy than the ordered by $0.15 \pm 0.05 k_{\mathrm{B}} /$ atom at $300 \mathrm{~K}$.

A method for neutron weight correction of solid solutions is described in section 13.2 , and an early version of this approach was used to correct the phonon DOS curves in Fig. 16 [188, 200, 201]. The most prominent effect of disorder is a shift of intensity from peaks at 21 and $25 \mathrm{meV}$ to lower energies, increasing the vibrational entropy of disordered $\mathrm{Cu}_{3} \mathrm{Au}$ by about $0.15 k_{\mathrm{B}}$ /atom, in general agreement with the calorimetry results [195]. Other phonon spectra [200], and computational work by Ozolins, Wolverton and Zunger [202], also showed this change in the spectrum at $21 \mathrm{meV}$ owing to changes optical modes (or local oscillations) emphasizing $\mathrm{Cu}$ atoms.

Using the force constants of $\mathrm{L1}_{2}$-ordered $\mathrm{Cu}_{3} \mathrm{Au}$, the motions of atoms in the different modes can be identified. The highest frequency modes around $25 \mathrm{meV}$ involve the motions of $\mathrm{Cu}$ atoms against $\mathrm{Au}$ atoms. The $\mathrm{Cu}$-atom motions were found primarily in the modes around $21 \mathrm{meV}$, and are associated with local $\mathrm{Cu}$ atom movements with less response from each $\mathrm{Au}$ neighbor. In some of these modes, a $\mathrm{Cu}$ atom moves perpendicular to the face of the of the standard $\mathrm{L}_{2}$ structural cube. The constraints on the motions of $\mathrm{Cu}$ atoms from the framework of the massive $\mathrm{Au}$ atoms, a characteristic of the $\mathrm{L}_{2}$ structure of $\mathrm{Cu}_{3} \mathrm{Au}$, could be removed in the disordered alloy. This may cause a shift of the modes at $21 \mathrm{meV}$ to lower energy. Although we do not have a definitive picture of the lattice dynamics of the disordered

\footnotetext{
39 Note that when $S_{\mathrm{vib}}^{\mathrm{Cu}}(x)=S_{\mathrm{vib}}^{\mathrm{Au}}(x), S_{\mathrm{vib}}=S_{\mathrm{vib}}^{\mathrm{nw}}$.
} 
alloys, the phonon DOS curves shown in Fig. 16 are consistent with this change in constraints of $\mathrm{Cu}$ atom motions.

A resonance mode associated with the damped motions of the massive Au atoms is a prominent feature of the phonon DOS of disordered $\mathrm{Cu}-\mathrm{Au}$ solid solutions with low $\mathrm{Au}$ concentrations. The broad resonance mode is centered at approximately $9 \mathrm{meV}$, and dominates the Au partial phonon DOS over a range of compositions $[201,203]$. The resonance mode seems responsible for a more rapid rise in vibrational entropy as $\mathrm{Au}$ is added to $\mathrm{Cu}$, as compared to adding $\mathrm{Cu}$ to $\mathrm{Au}$. Unfortunately the study of Au-rich alloys by neutron scattering is difficult owing to absorption, and this work is incomplete.

Finally, we return to the observations of Flinn, McManus, and Rayne [191], who measured no difference in the elastic constants of ordered and disordered $\mathrm{Cu}_{3} \mathrm{Au}$. From both theoretical and experimental lattice dynamics studies, it is also found that ordered and disordered $\mathrm{Cu}_{3} \mathrm{Au}$ have minimal differences in the low-frequency phonons that determine the elastic constants. The higher frequency phonons are responsible for the larger vibrational entropy of disordered $\mathrm{Cu}_{3} \mathrm{Au}$. Clearly it is risky to use Debye temperatures from elastic constant measurements to assess differences in vibrational entropy of alloy phases.

\subsection{2. $\mathrm{Pt}_{3} \mathrm{Fe}$}

To further explore the effects of local environments on the phonon DOS of the disorder-L $1_{2}$ order transition, a nuclear resonant inelastic x-ray scattering (NRIXS) experiment was performed on samples of $\mathrm{Pt}_{3}^{57} \mathrm{Fe}[204]$ to obtain a phonon partial DOS of $\mathrm{Fe}$ atoms. In the $\mathrm{L}_{2}$ structure of $\mathrm{Pt}_{3} \mathrm{Fe}$, all 12 first-nearest-neighbors surrounding a relatively light $\mathrm{Fe}$ atom are massive $\mathrm{Pt}$ atoms. The structural rearrangements of chemical disordering will vandalize this framework around the Fe atoms, so their vibrational frequencies are expected to change. In $\mathrm{L}_{2}$-ordered $\mathrm{Pt}_{3} \mathrm{Fe}$, the phonon partial DOS of Fe atoms is largely a single peak at $25 \mathrm{meV}$, approximately an Einstein mode. Figure 17 shows that with disorder, approximately $10 \%$ of the intensity of this peak is transferred to a broader peak around $19 \mathrm{meV}$. This transfer of intensity depends primarily on the short-range order in the alloy, suggesting that local environments dominate the changes in the phonon partial DOS.

A first interpretation of the spectra in Fig. 17 is that there are two vibrational environments for ${ }^{57} \mathrm{Fe}$. There is one subspectrum at $25 \mathrm{meV}$ associated with a nearlyfull complement of Pt first-nearest-neighbors, and a second subspectrum at $19 \mathrm{meV}$ associated with enough $\mathrm{Fe}$ first-neighbors to vandalize the cage of $\mathrm{Pt}$, and shift the vibrational frequency of the central $\mathrm{Fe}$ atom. If the disordered solid solution is a random solid solution, the numbers of different Fe environments can be calculated with a binomial probability distribution. From the areas of the two vibrational subspectra, the vandalized cage occurs with approximately 5 or more nearest neighbors. Of course the actual situation with the local vibrations is expected to involve the mutual positions of the different $\mathrm{Pt}$ atoms about the Fe atom, not just the total number of Pt atoms in a first-neighbor shell. Nevertheless, the results of Fig. 17 suggest that the concept of a cage structure is relatively robust, and more than one or two misplaced atoms are required for a significant change in the vibrational frequencies of the Fe atoms. 


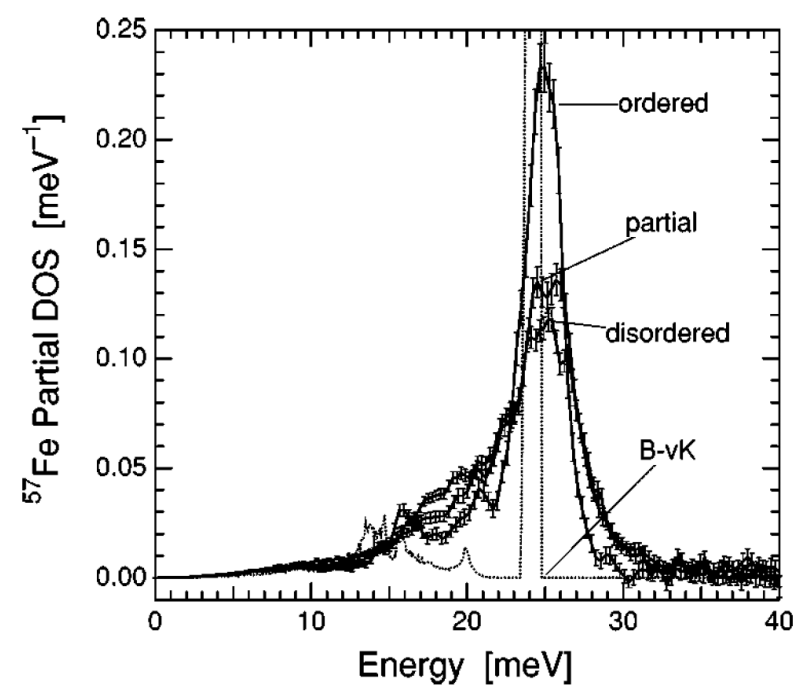

Figure 17. Phonon partial DOS curves for ${ }^{57} \mathrm{Fe}$ in $\mathrm{Pt}_{3}^{57} \mathrm{Fe}$ with $\mathrm{L}_{2}$ order (ordered), with short-range order but little long-range order (partial), and prepared by piston-anvil quenching without any measurable short-range order (disordered) [204]. Also shown is a curve calculated from a Born-von Kármán model (B-vK, which extends vertically to 1.8) [205].

\subsubsection{Formation Entropy of $\mathrm{L}_{2}$ Compounds}

Further information about the vibrational entropy of $\mathrm{L}_{2}$ compounds is obtained by comparing the vibrational entropy of the compound to the vibrational entropies of the pure elements from which it is formed. The advantage of this comparison is that the phonon DOS curves of the pure elements are usually available, and are generally accurate. The systematics of the calculated "entropy of formation," $\Delta S_{\mathrm{vib}}^{\text {form }}$, gives insight into the ordered structure, which helps our understanding of the order-disorder transformation itself.

From phonon DOS curves published in the literature, or from DOS curves obtained from a Born-von Kármán model with published force constants, the hightemperature limit of the vibrational entropy was obtained with Eq. 16 [206]. In this expression, the DOS $g^{\alpha}(\varepsilon)$ was the DOS of the ordered $\mathrm{L}_{2}$ compound, with composition $\mathrm{A}_{0.75} \mathrm{~B}_{0.25}$, and $g^{\beta}(\varepsilon)$ was the weighted sum of the DOS curves of elements $\mathrm{A}$ and $\mathrm{B}, 0.75 g^{\mathrm{A}}(\varepsilon)+0.25 g^{\mathrm{B}}(\varepsilon)$. A number of correlations were explored between $\Delta S_{\text {vib }}^{\text {form }}$ and the properties of the constitutent atoms [206], but the correlation shown in Fig. 18 was most successful.

The lattice parameter of the standard cube of the $\mathrm{L}_{2}$ structure is usually similar to the lattice parameter of the fcc unit cell of the majority species. Figure 18 shows that when the minority species is larger than the majority species, there is a significant decrease in the vibrational entropy of formation. This originates with an overall stiffening of phonons, i.e., a shift of the $\mathrm{L} 1_{2}$ spectrum to higher energies with respect to the average of the pure elements. On the other hand, inserting a smaller minority species between large majority atoms seems to cause only a modest softening of phonons in the $\mathrm{L}_{2}$ structure, and this is not such a strong function of the difference in metallic radius. The systematics of Fig. 18 support the concept of a caged minority species in the $\mathrm{L}_{2}$ structure - when the minority species is large and 


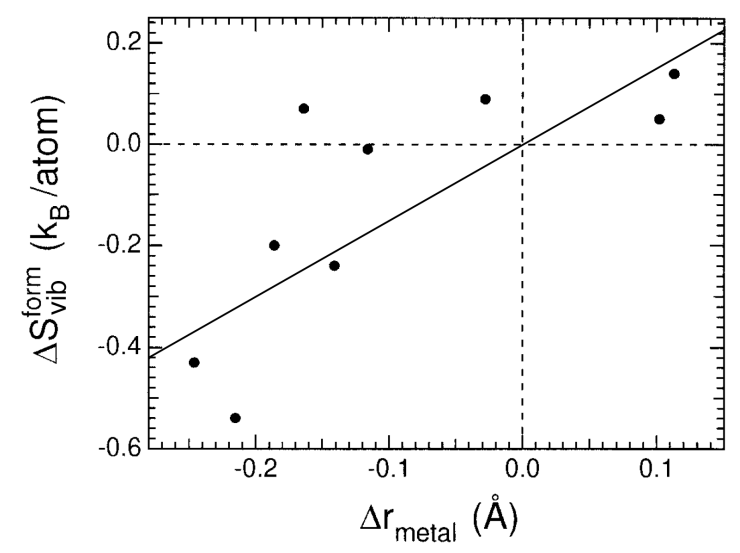

Figure 18. Vibrational entropies of formation of $\mathrm{L}_{2}$ alloys as a function of difference in metallic radius between the majority species and the minority species. The difference $\Delta r_{\text {metal }}$ is positive when the majority species is bigger than the minority species [206]. Line is from the second term of Eq. 124.

"squeezed," the phonon frequencies are increased. An intuitive picture is that the atoms are slightly-compressible spheres, which have higher interatomic forces when they are compressed substantially.

The extended bond proportion model of Section 5.2 gives a semiquantitative interpretation of the trend in Fig. 18. In the $\mathrm{L}_{2}$ structure, half of the $1 \mathrm{NN}$ pairs are $\mathrm{A}-\mathrm{B}$, half are $\mathrm{A}-\mathrm{A}$, and there are no $\mathrm{B}-\mathrm{B} 1 \mathrm{NN}$ pairs. Because the lattice parameters of the $\mathrm{L}_{2}$ compounds are similar to those of fcc $\mathrm{A}$, we assume that the $1 \mathrm{NN} \mathrm{A-A}$ bonds between majority species are unaffected by the minority solute atoms (and we need not consider changes in the B-B bond length, only elimination of B-B bonds during $\mathrm{L} 1_{2}$ compound formation). The vibrational entropy of formation per atom, $S_{\text {vib }}^{\mathrm{fL} 1_{2}}$, is (c.f., Eq. 52 et seq., and Eq. 68)

$$
\begin{aligned}
S_{\text {vib }}^{\mathrm{f} \mathrm{L1} 1_{2}} & =\left[S_{\text {vib }}^{\mathrm{L} 1_{2}}\right]-\left[\frac{3}{4} S_{\text {vib }}^{\mathrm{A}}+\frac{1}{4} S_{\text {vib }}^{\mathrm{B}}\right], \\
S_{\mathrm{vib}}^{\mathrm{f} \mathrm{L1} 1_{2}} & =\left[\frac{1}{2} 3 k_{\mathrm{B}} \ln \left(\frac{k_{\mathrm{B}} T}{\hbar \omega_{\mathrm{AA}}^{0}}\right)+\frac{1}{2} 3 k_{\mathrm{B}} \ln \left(\frac{k_{\mathrm{B}} T}{\hbar \omega_{\mathrm{AB}}^{0}\left(1-\gamma \delta V / V_{0}\right)}\right)\right] \\
& -\left[\frac{3}{4} 3 k_{\mathrm{B}} \ln \left(\frac{k_{\mathrm{B}} T}{\hbar \omega_{\mathrm{AA}}^{0}}\right)+\frac{1}{4} 3 k_{\mathrm{B}} \ln \left(\frac{k_{\mathrm{B}} T}{\hbar \omega_{\mathrm{BB}}^{0}}\right)\right], \\
S_{\mathrm{vib}}^{\mathrm{f} \mathrm{L} 1_{2}} & =\frac{3}{4} k_{\mathrm{B}} \ln \left(\frac{\omega_{\mathrm{AA}}^{0} \omega_{\mathrm{BB}}^{0}}{\left(\omega_{\mathrm{AB}}^{0}\right)^{2}}\right)+\frac{3}{2} k_{\mathrm{B}} \gamma \frac{\delta V}{V_{0}} .
\end{aligned}
$$

where the superscript " 0 " denotes the value for the pure elements or for $\Delta r_{\text {metal }}=0$. The $\Delta V / V_{0}$ of Eq. 123 can be obtained approximately from the $\Delta r_{\text {metal }}$ of Fig. 18 as $\Delta V / V_{0} \simeq 3 \Delta r_{\text {metal }} / 2 r_{0}$, where $2 r_{0} \simeq 3 \AA$, and assuming $\gamma=2$

$$
S_{\mathrm{vib}}^{\mathrm{f} \mathrm{L} 1_{2}} \simeq \frac{3}{4} k_{\mathrm{B}} \ln \left(\frac{\omega_{\mathrm{AA}}^{0} \omega_{\mathrm{BB}}^{0}}{\left(\omega_{\mathrm{AB}}^{0}\right)^{2}}\right)+1.5 k_{\mathrm{B}} \Delta r_{\text {metal }}[\AA]
$$

The second term in Eq. 124 gives the dependence on $\Delta r_{\text {metal }}$, and is shown as the diagonal line in Fig. 18. The first term in Eq. 124, the prediction from the bond 
proportion model (cf., last term in Eq. 61), should cause the line to be offset from the origin. This first term is also expected to cause deviations from the line.

\subsubsection{Mass and Volume Effects in Intermetallic Compounds}

It is interesting to compare changes in the phonon DOS when one of the atomic species in an ordered compound is replaced with atoms of a different element. After this substitution, the vibrational entropy is expected to decrease with force constant, increase with mass, and increase with lattice parameter. All such effects could be present simultaneously, and in different amounts for different alloy systems, so rigorous predictions are challenging. Experimental phonon spectra were used to test these expected correlations when the minority atoms were replaced and the crystal structure was largely unchanged [206]. Changes in vibrational entropy were obtained when the minority B-atoms in $\mathrm{A}_{3} \mathrm{~B} \mathrm{L1} 1_{2}, \mathrm{~A}_{3} \mathrm{~B} \mathrm{~A} 15$, and $\mathrm{A}_{2} \mathrm{~B}$ Laves phase compounds were replaced by a different species. The $\Delta S_{\text {vib }}$ showed a fair correlation to the expected effects of a change in the mass of the minority species $\left(3 / 2 c k_{\mathrm{B}} \ln \left(m_{1} / m_{2}\right)\right)$, when added to the effects expected from the change in unit cell volume (cf., Eq. 66). A precise correlation is not expected, however, in part because the interatomic distances do not track the change in volume of the unit cell.

An example of such a change in vibrational entropy with minority species substitution is shown in Fig. 19 [207]. Figures 19a and $19 \mathrm{~b}$ show the ${ }^{57} \mathrm{Fe}$ phonon partial DOS of $\mathrm{L}_{2}$-ordered $\mathrm{Pt}_{3}^{57} \mathrm{Fe}$ and $\mathrm{Pd}_{3}^{57} \mathrm{Fe}$, together with curves calculated with force constants from prior inelastic neutron scattering experiments [205, 208]. The atoms $\mathrm{Pt}$ and $\mathrm{Pd}$ have many chemical similarities, so it might be expected that the interatomic forces do not change significantly with the substitution of $\mathrm{Pd}$ for Pt. Figure 19c shows the result of a Born-von Kármán calculation that used the force constants for Pt, but the mass was changed to that of Pd (from 195 to 106). There is a semi-quantitative agreement with the actual ${ }^{57} \mathrm{Fe}$ phonon partial DOS of $\mathrm{L}_{2}$-ordered $\mathrm{Pd}_{3}^{57} \mathrm{Fe}$, suggesting the importance of the mass itself, but quantitative predictions of vibrational entropy are risky in this system, which undergoes an Invar transition under pressure.

\section{Unmixing}

\subsection{Computational Studies of Unmixing}

The transformation from a disordered solid solution to two phases of distinct chemical composition causes major changes in local chemistry, and substantial changes in configurational entropy. Nevertheless, there can be a large change in vibrational entropy when forming a disordered solid solution (Section 4.1). There are challenges in calculating phonons in disordered solid solutions. In first-principles electronic structure studies by Ozolins, Wolverton and Zunger [209], effects of vibrational entropy were deduced (and effects on the miscibility gaps were assessed), by subtracting a calculated configurational entropy from an experimental total entropy of formation. This left a residual entropy of formation that was probably vibrational. For the formation of disordered equiatomic $\mathrm{Ni}$ - $\mathrm{Au}$ from pure $\mathrm{Ni}$ and pure $\mathrm{Au}$, the vibrational entropy of formation was $+0.48 k_{\mathrm{B}}$ /atom. For a solid solution of $\mathrm{Cu}-\mathrm{Ag}$, the vibra- 


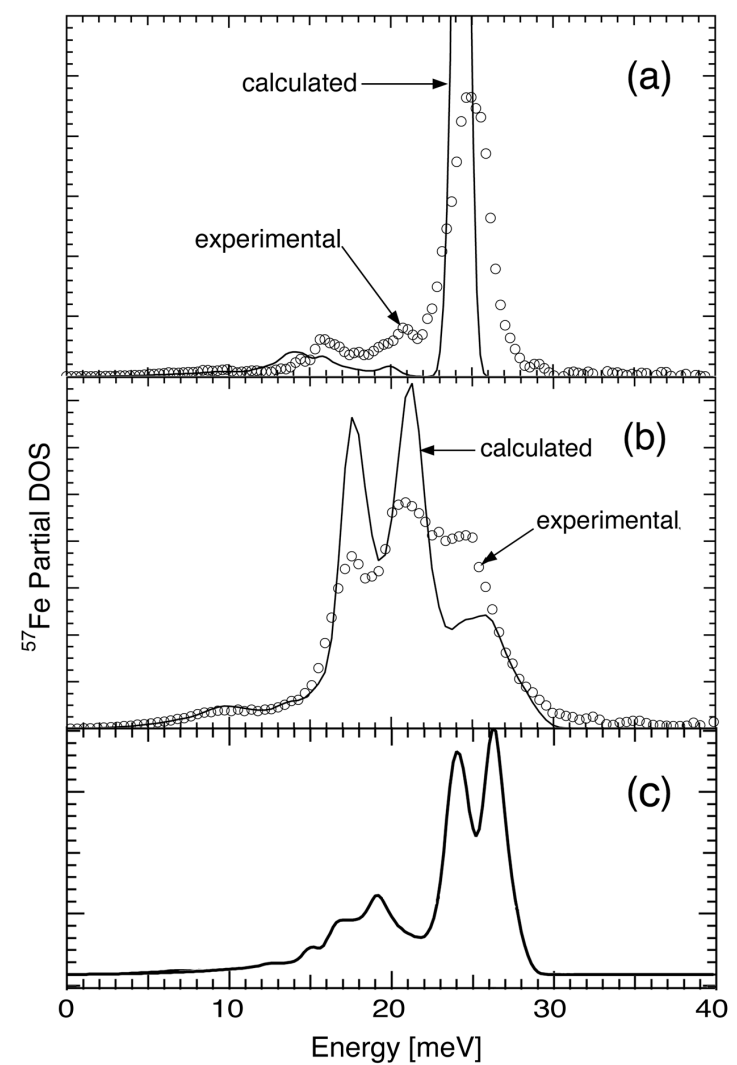

Figure 19. (a) Phonon partial DOS curves for ${ }^{57} \mathrm{Fe}, g_{F e}(\varepsilon)$, for $\mathrm{L} 1_{2}$-ordered $\mathrm{Pt}_{3}^{57} \mathrm{Fe}$, measured by NRIXS and calculated using force constants of [205]. (b) Phonon partial DOS curves for ${ }^{57} \mathrm{Fe}$, $g_{\mathrm{Fe}}(\varepsilon)$, for $\mathrm{L}_{2}$-ordered $\mathrm{Pd}_{3}^{57} \mathrm{Fe}$, measured by NRIXS and calculated using force constants of [208]. (c) Phonon partial DOS curves for ${ }^{57} \mathrm{Fe}, g_{F e}(\varepsilon)$, for $\mathrm{L}_{2}$-ordered $\mathrm{Pd}_{3}^{57} \mathrm{Fe}$, calculated by using the force constants of $\mathrm{Pt}_{3}^{57} \mathrm{Fe}$ [205] and the mass of Pd. After [207].

tional entropy of formation was $+0.37 k_{\mathrm{B}} /$ atom. These are large contributions to the thermodynamics of unmixing. The authors suggested that the atomic size mismatch could be responsible for such large effects. From a computational study on unmixing in the Ni-Cr system, there was a suggestion that the discrepancy between phase boundaries between experiment and first-principles calculations without vibrational entropy might be useful for identifying the importance of vibrational entropy [210].

Zarkevich, Tan, and Johnson used first-principles methods to study the unmixing phase diagram in the alloy systems $\mathrm{Ca}-\mathrm{Sr}, \mathrm{Au}-\mathrm{Pt}$, $\mathrm{Rh}-\mathrm{Pd}$, $\mathrm{Rh}-\mathrm{Cu}$, Rh- $\mathrm{Ag}$, and $\mathrm{Rh}-$ $\mathrm{Au}$ [211]. They also tested whether simpler methods could explain many of the trends, such as whether $T_{\text {crit }}$ could be predicted from a simple ratio of enthalpy to entropy. They discussed the difficulty of including vibrational entropy in simple estimates, but they showed some success with scaling the effects of vibrational entropy with differences in electronegativity of the elemental constituents. It may be difficult to use differences of electronegativity for quantitative estimates of the effects of vibrational entropy, especially for concentrated alloys, but it does seem that elements with large differences in electronegativity may show large effects of vibrational entropy in unmixing transformations. 
Burton, van de Walle and Kattner performed first principles computational studies of unmixing of three quasi-binary systems with the wurtzite structure, AlN-GaN, GaN-InN, and AlN-InN, using the bond-stiffness versus bond-length model [212]. With similar structures, there is a general expectation that the solubility should depend on differences in the ionic size of the metal atoms. The critical temperature for unmixing did correlate with the difference in ionic radii, but the effects of vibrational entropy did not. For example, although the phonon frequencies are expected to increase rapidly when larger In atoms are substituted on $\mathrm{Al}$ atom sites, the effects of vibrational entropy on the miscibility gap were surprisingly symmetrical in composition. This may be a consequence of the wurtzite structure, which is not close packed and may allow more local relaxations.

\subsection{Cluster Expansion Method for Solid Solutions}

A phonon DOS, a function of energy, pertains to a specific configuration of atoms. The interatomic forces are relatively short-ranged, so we expected that specifying local configurations of atoms would be sufficient to account for the phonon DOS. The cluster expansion method is a systematic way to construct a phonon DOS by summing contributions from different local clusters of atoms, starting with the chemical composition, and adding contributions from clusters of increasingly larger size. The cluster expansion method, which is extensible to DOS functions [213], is most useful when the phonon DOS can be accounted for with relatively few terms.

A phonon DOS $g_{j}(\varepsilon)$ is related to the local atom arrangements in a structure (the structure is designated $j$ ) through "interaction phonon DOS" functions, $\left\{G_{0}(\varepsilon), G_{1}(\varepsilon), G_{2}(\varepsilon) \ldots G_{\nu-1}(\varepsilon)\right\}$, where $\nu$ is the number of terms in the cluster expansion. The ordered set of interaction DOS functions can be written as a vector, $\vec{G}(\varepsilon)$, so

$$
g_{j}(\varepsilon)=\vec{G}(\varepsilon) \vec{\xi}_{j},
$$

where $\vec{\xi}_{j}$ is a set of atom correlation functions, $\left\{\xi_{j}\right\}$, in the specific composition or structure, $j$. With DOS curves from a set of different compositions or structures, $\vec{g}(\varepsilon)=\left\{g_{1}(\varepsilon), g_{2}(\varepsilon) \ldots\right\}$, again written as a vector, we can solve for a set of different interaction DOS functions using an inversion method of Connolly and Williams [214],

$$
\vec{G}(\varepsilon)=\vec{g}(\varepsilon) \underline{\xi}^{-1}
$$

where the matrix $\xi^{-1}$ is obtained from the correlation functions below.

The correlation functions $\left\{\xi_{j 1}, \xi_{j 2}, \ldots\right\}$ are obtained by geometrical analysis of atom positions in each structure, $j$. Specifically, for a cluster composed of $n$ atoms:

$$
\xi_{j n}=\frac{1}{N_{n}} \sum_{i=1}^{n} \sigma_{p_{i}},
$$

where $p_{i}$ are specific sites. Equation 127 is a product of "spin variables," where $\sigma=+1$ for an A-atom and $\sigma=-1$ for a B-atom in a binary alloy. Consider, for 
example, correlation functions for a disordered solid solution, where each site has the probability of $x_{\mathrm{B}}$ of being occupied by a B-atom, and $1-x_{\mathrm{B}}$ by an A-atom. The average for $n=1$ is $\xi_{1}=\sigma_{B} x_{\mathrm{B}}+\sigma_{A}\left(1-x_{\mathrm{B}}\right)=1-2 x_{\mathrm{B}}$. For clusters of two atoms (i.e., pairs), both sites are occupied randomly by A- or B-atoms, so $\xi_{2}=\left(1-2 x_{\mathrm{B}}\right)^{2}$, and for an $n$-atom cluster in a random solid solution $\xi_{n}=\left(1-2 x_{\mathrm{B}}\right)^{n}$. For an equiatomic alloy with $x_{\mathrm{B}}=0.5, \xi_{n}=0$ for all $n$ except $n=0$. For $n=0$, the interaction phonon DOS, $G_{0}(\varepsilon)$, is the phonon DOS of the equiatomic solid solution, and all other $G_{n}(\varepsilon)$ are corrections to this function for deviations from the equiatomic. The cluster functions $G_{n}(\varepsilon)$ contribute to the DOS in proportion to how many $n^{\text {th }}$ clusters there are in a disordered alloy of composition $c$, compared to how many there are in an equiatomic disordered alloy.

The same systematics can be used for the phonon partial DOS functions of Aand B-atoms. Here the index $d$ denotes either "A" or "B." The analogs of Eqs. 125 and 126 are

$$
\begin{aligned}
\vec{g}_{d, j}(\varepsilon) & =\vec{G}_{d}(\varepsilon) \xi_{d, j} \\
\vec{G}_{d}(\varepsilon) & =\vec{g}_{d}(\varepsilon) \underline{-}_{-}^{-1}
\end{aligned}
$$

Neutron weighting (Sect. 8.3) is obtained with the phonon partial DOS (PDOS) functions ${ }^{40}$

$$
g_{j}^{\mathrm{nw}}(\varepsilon)=\sum_{d} \frac{x_{\mathrm{A} j} \sigma_{\mathrm{A}}}{M_{\mathrm{A}}}\langle M / \sigma\rangle g_{d, j}(\varepsilon),
$$

where each contribution to $g_{j}^{\mathrm{nw}}(\varepsilon)$ (for the composition or structure $j$ ) is weighted by the phonon scattering efficiency of its A- and B-atoms and their partial DOS $g_{d, j}(\varepsilon)$. The constant $\langle M / \sigma\rangle$ provides normalization. It can be shown that the neutronweighted interaction DOS functions can be written

$$
\vec{G}^{\mathrm{nw}}(\varepsilon)=\sum_{d} R_{-} C_{-} \xi_{-}^{-1} g_{d}(\varepsilon)
$$

where the matrix $C_{d}$ accounts for the neutron weighting [188, 215]. The matrix $R_{d}$ relates the interaction DOS (IDOS) functions to the interaction partial DOS (IPDOS) function of species $d(\mathrm{~A}$ or $\mathrm{B})$

$$
\vec{G}(\varepsilon)=\sum_{d} R_{-} \vec{G}_{d}(\varepsilon) .
$$

The $R_{d}$ is obtained from the correlation functions of the disordered solid solution.

For a disordered solid solution, Lucas, et al. [188, 215, 216] found strategies to solve for the phonon partial DOS functions from neutron-weighted DOS curves of

\footnotetext{
40 There are two subtleties: 1) It is assumed that the Debye-Waller factors for the two species of atoms do not differ significantly. This assumption should be adequate at low temperatures. 2) If the phonon spectra from all structures $\{j\}$ do not span the same range of energy, the energy can be renormalized to the highest energy mode in each structure. This offers a good comparison of partial DOS functions, but requires further assessments for the entropy.
} 
solid solutions. Arguably the best method is to obtain sets of experimental data with differences in the phonon scattering cross sections of the different species, such as with Fe alloys studied by neutron scattering and with ${ }^{57} \mathrm{Fe}$ inelastic nuclear resonant x-ray scattering (NRIXS). Alternative approaches can be developed with the assumption that the partial DOS of the host atoms is unaltered by the solute atoms, but this assumption can be violated if the solute atoms alter the electronic band structure or band filling. Improvements in the statistical quality of experimental neutron-weighted phonon DOS functions offer other opportunities for comparing samples with rather similar chemical compositions, and further development of these methods is expected with improvements in neutron instrumentation.

\subsection{Fe-Cr Solid Solutions}

Using the cluster expansion method of the previous section, Lucas, et al., obtained a high degree of detail about how the vibrational entropy depends on local configurations of atoms in bcc Fe-Cr $[188,215]$. The $n=0$ term, $G_{0, \mathrm{Cr}}(\varepsilon)$, is the Cr partial DOS of a random solid solution of $\mathrm{Fe}_{50} \mathrm{Cr}_{50}$. The $n=1$ term gives the effect on the PDOS of having different numbers of $\mathrm{Fe}$ and $\mathrm{Cr}$ atoms in the nearest-neighbor $(\mathrm{NN})$ shell of the $\mathrm{Cr}$ atom. ${ }^{41}$ The function $G_{1, \mathrm{Cr}}(\varepsilon)$ is added to $G_{0, \mathrm{Cr}}(\varepsilon)$ when the atom in the $\mathrm{NN}$ shell of the $\mathrm{Cr}$ atom is $\mathrm{Cr}$, and subtracted when it is Fe. Figure 20 shows that the effect of $\mathrm{Cr}$ atoms in the $\mathrm{NN}$ shell of a $\mathrm{Cr}$ atom is to decrease the number of modes in the ranges of $0 \leq \varepsilon \leq 0.59$ and $0.83 \leq \varepsilon \leq 1$, while increasing the number of modes in the range $0.59 \leq \varepsilon \leq 0.83$. The triangle term $\mathrm{n}=2$ gives the effect of the like or unlike pairs of atoms within the NN shell of a $\mathrm{Cr}$ atom. The function $G_{2, \mathrm{Cr}}(\varepsilon)$ adds to both $G_{0, \mathrm{Cr}}(\varepsilon)$ and $G_{1, \mathrm{Cr}}(\varepsilon)$ when a pair in the NN shell of the $\mathrm{Cr}$ atom is composed of two of the same atoms. Pairs of like atoms decrease the number of modes in the ranges $0 \leq \varepsilon \leq 0.56,0.80 \leq \varepsilon \leq 0.88$, and $0.94 \leq \varepsilon \leq 1$ while increasing the number of modes in the remaining ranges, whereas unlike pairs do the opposite.

The IPDOS functions can be used to construct the PDOS curves of $\mathrm{Fe}$ and $\mathrm{Cr}$ atoms in alloys (Fig. 21). These PDOS curves can then be used to construct the neutron-weight-corrected DOS curves. The neutron-weight correction stiffens all of the DOS curves because the Fe PDOS curves have more low-energy modes than the Cr PDOS curves, especially below $\varepsilon=0.7$, but Fe atoms are more efficient scatterers. For Fe-Cr alloys, the PDOS curves of both Fe and $\mathrm{Cr}$ atoms undergo an average softening upon alloying, giving a net positive vibrational entropy of mixing. The softening of the Cr PDOS curves is larger, so at low concentrations of $\mathrm{Fe}$ in $\mathrm{Cr}$, the phonon entropy of mixing rises relatively rapidly with Fe concentration, giving a skewness to the concentration dependence of the vibrational entropy of mixing.

Cluster expansion methods were originally developed for thermodynamic quantities such as entropy, and the same notation is useful for interaction entropies of solid solutions, $s_{n, \mathrm{Fe}}^{\mathrm{vib}}$ and $s_{n, \mathrm{Cr}}^{\mathrm{vib}}$. For a random solid solution, the vibrational entropy of mixing is, in terms of the interaction phonon partial entropies

$$
\Delta S^{\mathrm{vib}}=-4 x_{\mathrm{Cr}}\left(1-x_{\mathrm{Cr}}\right)\left\{\left[s_{1, \mathrm{Cr}}^{\mathrm{vib}}+s_{1, \mathrm{Fe}}^{\mathrm{vib}}\right] / 2+x_{\mathrm{Cr}} s_{2, \mathrm{Cr}}^{\mathrm{vib}}+\left(1-x_{\mathrm{Cr}}\right) s_{2, \mathrm{Fe}}^{\mathrm{vib}}\right\}
$$

\footnotetext{
41 Lucas, et al., used a combined first and second nearest-neighbor shell of the bcc structure as the NN shell [188, 215].
} 


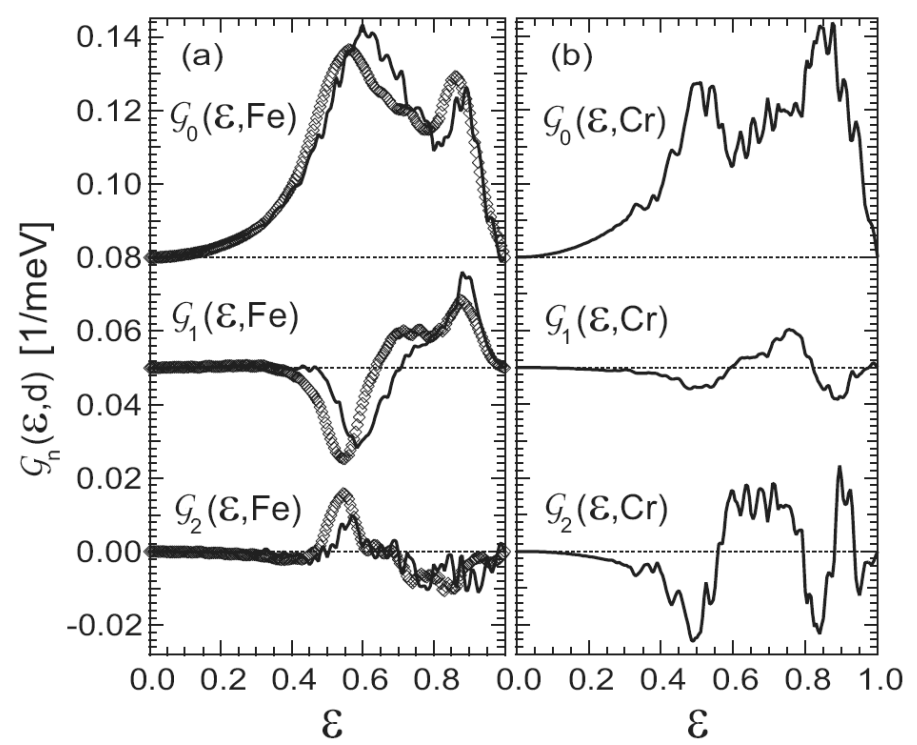

Figure 20. Interaction phonon DOS functions of (a) Fe and (b) $\mathrm{Cr}$ in bcc solid solutions of Fe- $\mathrm{Cr}$ alloys, determined from neutron scattering (solid lines), and direct measurement by ${ }^{57} \mathrm{Fe}$ NRIXS (points). The $n=1$ and $n=2$ curves are offset vertically. Note that the energy scale was renormalized to the energy of the highest energy mode in each structure.

The function $-4 x_{\mathrm{Cr}}\left(1-x_{\mathrm{Cr}}\right)$ is a parabola, scaled by the average $\left[s_{1, \mathrm{Cr}}^{\mathrm{vib}}+s_{1, \mathrm{Fe}}^{\mathrm{vib}}\right] / 2$. This average is negative, so the term introduces a positive entropy of mixing, with a maximum at $x_{\mathrm{Cr}}=0.5$. The remaining two terms associated with $s_{2, d}^{\mathrm{vib}}$ are the equation of a line, giving an asymmetry to the vibrational entropy of mixing when $s_{2, \mathrm{Cr}}^{\mathrm{vib}} \neq s_{2, \mathrm{Fe}}^{\mathrm{vib}}$. The vibrational entropy of mixing is shifted toward pure Cr because $G_{2, \mathrm{Cr}}(\varepsilon)<G_{2, \mathrm{Fe}}(\varepsilon)$ in the energy range 0 to $25 \mathrm{meV}$. The maximum critical temperature and composition of the miscibility gap in Fe-Cr are $905 \mathrm{~K}$ and $x_{\mathrm{Cr}}=0.51$, respectively. Assuming that the values of $s_{n, d}^{\mathrm{vib}}$ for $n>0$ do not change appreciably with temperature, Eq. 133 can be used to determine the effect of the vibrational entropy on the miscibility gap for spinodal decomposition. When the vibrational entropy of mixing in the bcc phase is removed, the miscibility gap for a random solution of $\mathrm{Fe}-\mathrm{Cr}$ is shifted up in temperature and toward pure $\mathrm{Cr}$, with a critical temperature and composition of $1268 \mathrm{~K}$ and $x_{\mathrm{Cr}}=0.61$, respectively $[188,215]$. It is known that the enthalpy of mixing is also shifted toward pure $\operatorname{Cr}[217,218,219,220]$, so the vibrational entropy counteracts the asymmetry of the enthalpy of mixing, especially at higher temperatures. In short, the vibrational entropy of mixing lowers the miscibility gap and shifts its maximum towards the equiatomic composition. 


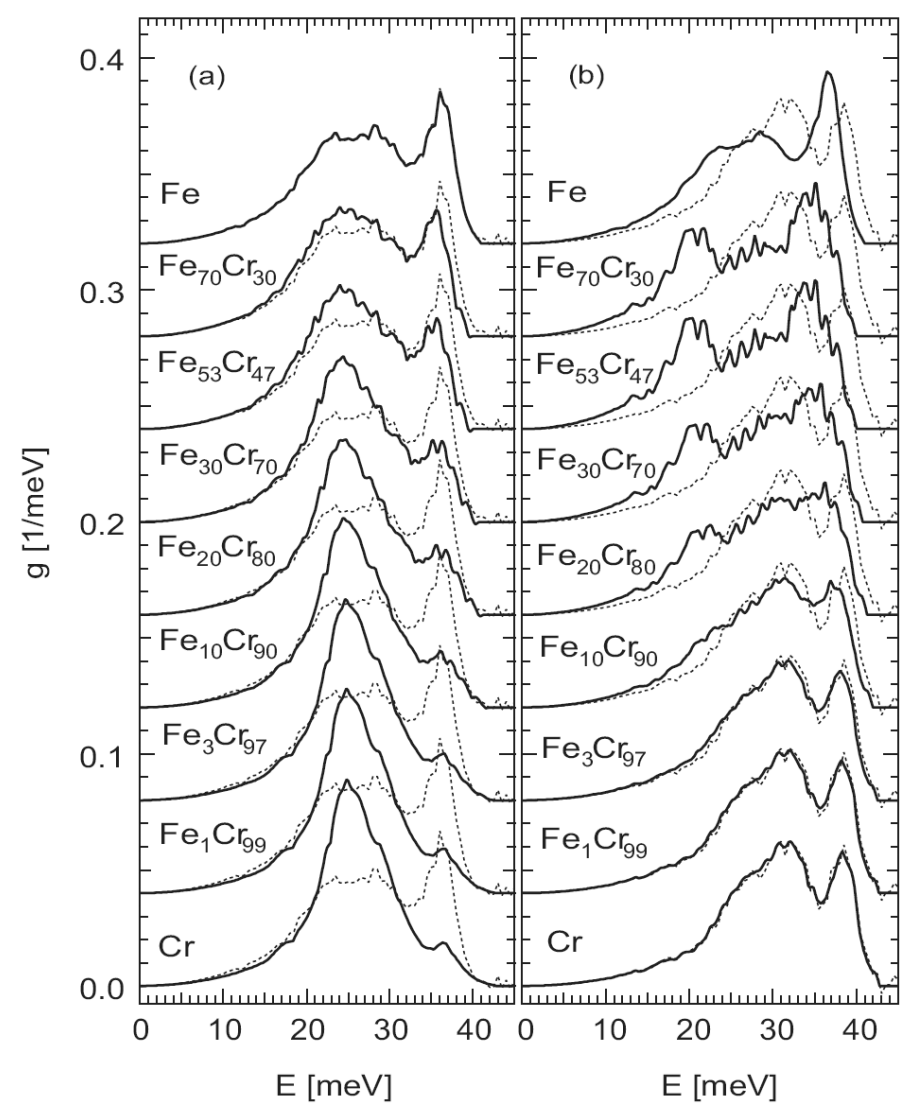

Figure 21. PDOS curves from the cluster expansion fitting method for the concentrations of the alloys measured: (a) Fe PDOS curves (solid lines), and measured pure Fe DOS (dashed line), and (b) Cr PDOS curves (solid lines), and measured pure Cr DOS (dashed line). After [188, 215].

\section{Interstitial Alloys}

\subsection{Interstitial OXYGen And Nitrogen}

Inserting small atoms into interstitial sites of a crystalline host matrix is sometimes treated as a simple problem of distributing the small atoms over a set of independent lattice sites. The energy of insertion of each atom is assumed a constant, and a configurational entropy can then be calculated readily with a form of Eq. 11, for example. The real situation is more complicated, owing to distortions of the matrix around each interstitial atom. Sometimes the elastic interactions between distorted unit cells cause structural organizations of the interstitial atoms. Anisotropic structures such as martensite in steel are formed this way, accompanied by large, long-range elastic distortions. These distortions are expected to alter the vibrational frequencies of both host and interstitial atoms, and the vibrational entropy. Such effects are not yet well understood.

Danilkin, et al., performed inelastic neutron scattering studies on $\mathrm{V}, \mathrm{Nb}$, and $\mathrm{Ta}$ with concentrations of up to 11 at.\% of interstitial $\mathrm{O}$ and $\mathrm{N}[221,222,223]$. They argued that the mass difference between the host and interstitial elements causes 
separation of the phonon DOS into bands dominated by the partial DOS of the metal atoms at low energies, and dominated by the partial DOS of the interstitial atoms at high energies. Although a full justification is not presented, it may be reasonable to neglect the contribution from the interstitial partial DOS in the low-energy part of the spectrum, and ascribe all changes to the phonon partial DOS of the metal atoms. With this assumption, large changes in vibrational entropy of the host metal are obtained. For example, in a $\mathrm{V}-3$ at.\%O alloy, the average vibrational frequency of the $\mathrm{V}$ atoms increases by approximately $6 \%$, which is a change of $+0.18 k_{\mathrm{B}} / \mathrm{V}$ atom with respect to pure $\mathrm{V}$. This is large compared to the configurational entropy of mixing for an alloy of 3 at.\% O, which from Eq. 11 is $-0.135 k_{\mathrm{B}} / \mathrm{O}$ atom. It is also interesting that these effects of interstitial atoms on the vibrations of host atoms are sub-linear at higher solute concentrations. Similar, although somewhat smaller, effects were found for nitrogen in vanadium, and for oxygen in niobium. For alloys of Ta-N, for which the mass difference is quite large, the effects were somewhat smaller, although the authors point out their concern that the measurements did not average the coherent scattering from Ta over a sufficiently wide range of $Q$.

\subsection{Hydrogen AND Hydrides}

Hydrogen and deuterium differ in mass by a factor of two. The characteristic vibrational frequencies of $\mathrm{H}_{2}$ and $\mathrm{D}_{2}$ molecules differ by almost exactly $\sqrt{2}$. Analogous mass effects are expected for hydrogen in a metal hydride. Owing to its low mass, hydrogen typically has high vibrational frequencies, which can show effects of quantized occupancy at temperatures well above room temperature because the Planck occupancy factor is still small. The V-H and the V-D phase diagrams have been measured, and the phase boundary between the disordered solid solution phase and the two-phase region is shifted to lower temperature for V-D by approximately $15 \%$ $[224] .{ }^{42}$ With deuterium there is a greater difference in the vibrational entropy of the solid solution compared to the unmixed state at lower temperatures, and this difference with respect to hydrogen seems understandable as a mass effect [225]. It has also been noted that with substitution of deuterium or tritium, the lattice parameter of $\mathrm{LiH}$ is reduced at low temperatures, although this difference decreases with temperature [226]. At high temperatures in the classical limit, it is expected that the mass effects on the thermal expansion of $\mathrm{LiH}$ versus LiT will disappear.

The alkali hydrides $\mathrm{LiH}$ and $\mathrm{NaH}$ both have the $\mathrm{B} 1(\mathrm{NaCl})$ structure at ambient pressure, but $\mathrm{NaH}$ transforms to the $\mathrm{B} 2(\mathrm{CsCl})$ structure at a pressure of $29 \mathrm{GPa}$, and $\mathrm{LiH}$ is predicted to transform to $\mathrm{B} 2$ at $308 \mathrm{GPa}$ [227]. A computational study of phonons in $\mathrm{B} 1 \mathrm{LiH}$ and $\mathrm{LiD}$ by $\mathrm{Yu}$, et al. [227], showed a large isotope effect on the three high-frequency optical branches that have a large partial DOS for $\mathrm{H}$ atom motions. A much smaller isotope effect was found for the three acoustic branches. The calculated heat capacity of $\mathrm{B} 1 \mathrm{LiH}$ was in good agreement with experimental measurements, which also reported that the Grüneisen parameters increase at lower temperatures [228]. Since the phonon spectrum extends to approximately $100 \mathrm{meV}$ for $\mathrm{LiH}$, the difference in the zero point energies of $\mathrm{LiD}$ and $\mathrm{LiH}$ at ambient temperature is important for the vibrational thermodynamics, and there are large

\footnotetext{
42 There are also differences in the phase fields of the V-H and V-D phase diagrams.
} 
zero-point effects on the lattice parameters and bulk moduli. The calculated phonon frequencies of $\mathrm{LiH}$ and $\mathrm{NaH}$ varied with volume, and these frequencies were used with the harmonic entropy expressions (i.e., a quasiharmonic approximation was used) to calculate the Helmholtz free energy. The calculated B1 to B2 transformation pressure for $\mathrm{NaH}$ was in good agreement with experimental measurement [227].

Equilibrium between a system containing a hydrogen sorbent in contact with a gas reservoir at fixed temperature and pressure ensures that the enthalpy of sorption, $\Delta H$, is dissipated as heat.

$$
\Delta H \equiv H_{\mathrm{g}}-H_{\mathrm{s}}=T\left(S_{\mathrm{g}}-S_{\mathrm{s}}\right) .
$$

with the subscript $\mathrm{g}$ for the full system with hydrogen in the gas, and $\mathrm{s}$ for the same system with hydrogen in or on the solid sorbent. The entropy of hydrogen gas is large, $S_{\text {gas }}=131 \mathrm{~J}(\mathrm{~mol} \mathrm{~K})^{-1}=7.9 k_{\mathrm{B}} /$ atom at STP. For the same pressure of gas, sorbents with larger $\Delta H$ tend to have higher operating temperatures.

A consequence of Eq. 134 is the release of heat during hydrogen sorption. Consider the case of lithium borohydride, for which $\Delta H=67 \mathrm{~kJ} /$ mole at $400^{\circ} \mathrm{C}$. The heat release from the sorption of 2500 moles of hydrogen is $167 \mathrm{MJ}$. For a refueling in 3 minutes, one megawatt of heat must be dissipated! Reducing this heat load requires either lower sorption temperatures, or a reduction of $S_{\mathrm{g}}-S_{\mathrm{s}}$. The degrees of freedom of diatomic hydrogen gas that contribute to $S_{\mathrm{g}}$ are translational, rotational, vibrational, electronic, and nuclear. The first two contributions are big, and generally unavailable to hydrogen in a solid, reducing the maximum value of $S_{\mathrm{s}}$. On the other hand, the vibrational frequency of the $\mathrm{H}_{2}$ molecule has a characteristic temperature of $6215 \mathrm{~K}$, suppressing its contribution to the entropy at ambient temperature. It is possible to get lower vibrational frequencies in compounds. Ozolins, Majzoub, and Wolverton [229] found effects of this sort, and suggested an approach for minimizing $S_{\mathrm{g}}-S_{\mathrm{s}}$. Storing hydrogen in complex anions such as $\mathrm{NH}_{2}$ and $\mathrm{BH}_{4}$ can produce crystal structures with low-frequency rotational modes, which increase the vibrational entropy of $S_{\mathrm{s}}$. After the hydrogen is removed, it is helpful if the solid has a low vibrational entropy owing to stiff phonons, further reducing $S_{\mathrm{g}}$. Nevertheless, with $S_{\text {gas }}=7.9 k_{\mathrm{B}} /$ atom, it is a challenge to decrease $S_{\mathrm{g}}-S_{\mathrm{s}}$ substantially.

\subsection{Lithium Intercalation AND InSERTion}

There are numerous electrochemical studies of the insertion and de-insertion of lithium in materials. A few of these studies have reported precise information on the chemical potential of the Li atoms, and how it changes with concentration. In equilibrium (i.e., steady-state without changing the charge of the electrochemical cell), the difference in voltage of the anode and cathode of a reliable electrochemical cell is precisely the difference in chemical potential of the $\mathrm{Li}$ atom between one electrode and the other. A counter-electrode of pure Li metal is especially useful for thermodynamic measurements, because there is no difference in the chemical potential for the different Li atoms as they are deposited on, or removed from, a pure Li metal electrode. By varying temperature, it is possible to measure the temperature dependence of the free energy per atom, and hence determine the 
entropy of intercalating additional Li into the material

$$
\left.\Delta S(x)=F \frac{\partial E_{0}}{\partial T}\right)_{x},
$$

where $F$ is the Faraday constant and $E_{0}$ is the equilibrium voltage of the cell. Although $E_{0}$ may be volts, and may change with temperature by only $0.1 \mathrm{mV}$ per degree $\mathrm{K}$, accurate measurements of $\Delta S(x)$ are practical with modern instrumentation and good electrochemical cells.

\subsubsection{Lithium Intercalation in Graphite}

Graphitic carbons are in widespread use as anode materials in rechargeable Li batteries. Under ambient conditions, Li atoms can be inserted into graphite to a concentration of $\mathrm{Li}_{1} \mathrm{C}_{6}$. Several intermediate phases are formed. As the concentration of $\mathrm{Li}, x$, increases in $\mathrm{Li}_{x} \mathrm{C}_{6}$, the first phase is a disordered distribution of $\mathrm{Li}$ atoms in the van der Waals gap between graphene planes. Figure 22 shows a large and positive entropy of insertion for small $x$. Assuming that all sites in the disordered phase are filled at $x=0.25$, the concentration dependence of Eq. 11 predicts the curved part of $\Delta S_{\text {calc }}$, which is in reasonable agreement with the experimental results for $\Delta S_{\text {meas }}$. Over this range of composition, $\Delta H_{\text {meas }}$ is reasonably constant, so perhaps a lattice gas model can successfully explain the intercalation thermodynamics.

It is known that further lithiation occurs in stages. A Stage II compound (with $\mathrm{Li}$ atoms filling alternate gaps between graphene planes) is formed up to the concentration $x=0.5$, followed by a Stage I compound (with Li atoms filling all gaps between graphene planes). These compounds form by first-order reactions, with an approximately constant enthalpy and entropy for each additional amount of transformed material. Figure 22 does show that $\Delta S_{\text {meas }}$ and $\Delta H_{\text {meas }}$ are fairly constant, with a break at the boundary of $x=0.5$ that marks the onset of Stage I compound formation.

Figure 22 shows that the entropies of formation of Stage II and Stage I compounds are negative. Experimental studies of the vibrational spectra of intercalated lithium atoms provide two Debye temperatures, a $\theta_{\mathrm{D} \perp} \simeq 893 \mathrm{~K}$ for the one dimension of lithium vibration normal to the graphene planes, and a $\theta_{\mathrm{D} \|} \simeq 392 \mathrm{~K}$ for the two dimensions of lithium vibrations in the plane of the graphene sheets [230]. With a Debye temperature $\theta_{\mathrm{D} 0} \simeq 380 \mathrm{~K}$ for bcc Li metal [231], the vibrational entropy of lithiation $\Delta S_{\text {calc }}$ is obtained as the flat line in Fig. 22. In essence, the vibrational entropy of intercalation is negative because the modes of Li vibration perpendicular to the graphene planes are highly constrained, and have high frequencies. The resulting negative vibrational entropy of intercalation dominates the entropy. There should be little change in configurational entropy when Stage I or Stage II compounds are formed because they are ordered.

\subsubsection{Lithium Insertion in $\mathrm{Li}_{x} \mathrm{CoO}_{2}$}

The layered hexagonal compound $\mathrm{Li}_{x} \mathrm{CoO}_{2}$ preserves a transition-metal oxide crystal framework as $\mathrm{Li}^{+}$ions are inserted or extracted. Because the chemical potential of the $\mathrm{Li}$ atom is lower by approximately $4 \mathrm{eV}$ in $\mathrm{Li}_{x} \mathrm{CoO}_{2}$ than in graphite, these oxides are excellent for service in rechargeable Li batteries. The "O3" hexagonal structure is stable over the composition range, $\sim 0.58<x \leq 1$, the approximate range used 


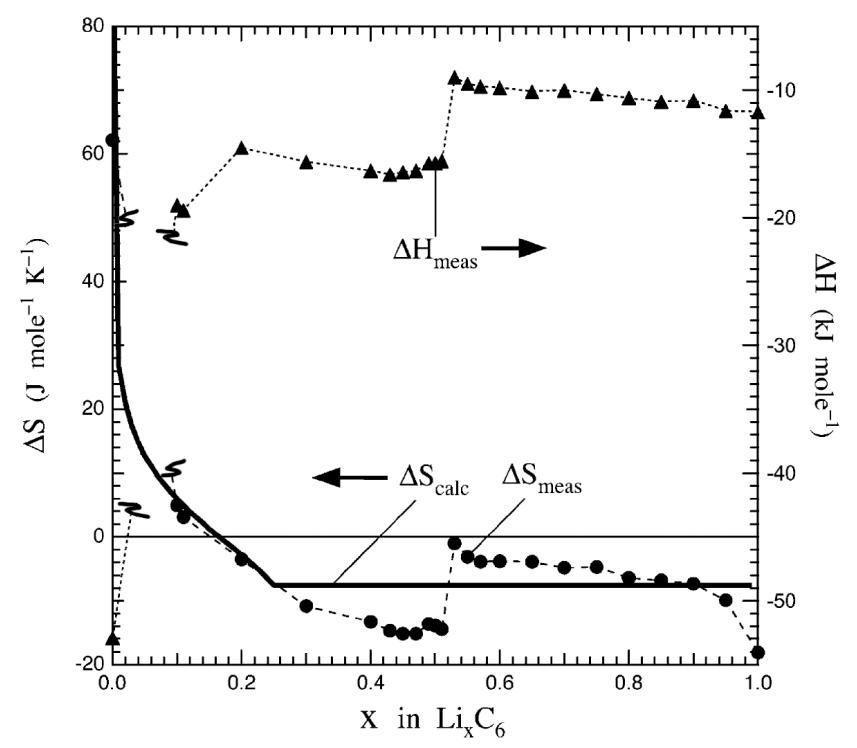

Figure 22. Composition-dependence of the entropy and enthalpy of intercalation, $\Delta S(x)$ and $\Delta H(x)$. Labels $\Delta S_{\text {meas }}$ and $\Delta H_{\text {meas }}$ refer to the experimental data. The label $\Delta S_{\text {calc }}$ refers to the calculation of the entropy of intercalation (see text). After [232].

in practice for rechargeable batteries. For $0.95<x \leq 1$, however, the O3 phase has a lattice parameter that is smaller than for $x=0.833$, and there is a region of twophase equilibrium between $x=0.833$ and $x=0.95$. There is a concomitant change from metal to insulator between $x=0.833$ and $x=0.95$. In the composition range $0.58<x<0.833$ there is a disordered distribution of Li atoms on the available sites, so the configurational entropy is expected to vary strongly with composition.

Phonon DOS measurements were performed on samples of $\mathrm{Li}_{x} \mathrm{CoO}_{2}$ for various values of $x$ in the O3 structure, including $x=0.62$ and $x=1.0$ [233]. Perhaps surprisingly, there was little difference in the DOS or the vibrational entropy of these two compositions. On the other hand, there is a considerable difference in the vibrational entropy of the $\mathrm{Li}$ in $\mathrm{Li}$ metal versus in $\mathrm{Li}_{x} \mathrm{CoO}_{2}$. Because the phonon frequencies of $\mathrm{Li}$ metal are much lower than those of $\mathrm{Li}_{x} \mathrm{CoO}_{2}$, as $\mathrm{Li}$ is transferred from metal to oxide, the vibrational entropy decreases. The contributions to the entropy of lithiation of $\mathrm{Li}_{x} \mathrm{CoO}_{2}$ are shown in Fig. 23. The data points were obtained with measurements that exploited Eq. 135. The smooth curve labeled $\Delta S_{\mathrm{cf}}$ was calculated from the configurational entropy of the disordered solid solution (it is the derivative $\partial S_{\text {cf }} / \partial x$ because the open circuit voltage measures the change in entropy as additional lithium is inserted in the material). In Fig. 23, $\Delta S_{\mathrm{cf}}$ was lowered vertically to make a best fit to the electrochemical results. This offset is mostly accounted for by the vibrational entropy of lithiation, $\Delta S_{\text {vib }}$, which is large and negative, approximately $-2.4 k_{\mathrm{B}}$ /atom, although it has only a small variation with $x$. (The correction from the electronic entropy, $\Delta S_{\mathrm{el}}$, is not large because there are few states near the Fermi level in these materials.) In summary, and in analogy to Li intercalation into graphite, the $\mathrm{Li}^{+}$inserted into $\mathrm{Li}_{x} \mathrm{CoO}_{2}$ is more stiffly constrained than in Li metal, giving higher vibrational frequencies and a lower vibrational en- 


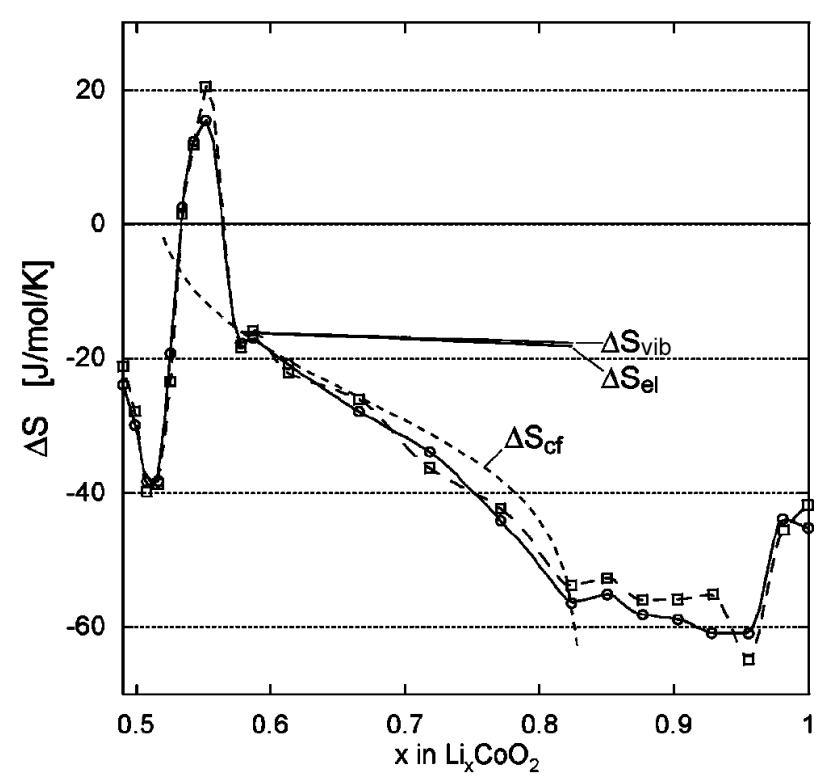

Figure 23. Configurational entropy $\Delta S_{\mathrm{cf}}$ (offset vertically), vibrational entropy $\Delta S_{\mathrm{ph}}$, (and electronic entropy $\Delta S_{\text {el }}$ correction) of the lithiation reaction in $\mathrm{Li}_{x} \mathrm{CoO}_{2}$ over the composition range $0.6<x<0.833$, superimposed on electrochemical data.

tropy. Since this does not change much with Li concentration, the dominant effects probably involve the $\mathrm{Li}$ and the host structure (rather than $\mathrm{Li}-\mathrm{Li}$ interactions).

\section{Martensite and Twinning}

Iron-carbon martensite is a classic interstitial solid solution. The deformation potential for $\mathrm{C}$ in bct $\mathrm{Fe}$ is of order $1 \mathrm{eV}$ - much higher than for most substitutional solid solutions [234]. With a carbon concentration of 4at.\%, the tetragonality of the martensite unit cell is nearly $4 \%$, which causes a large distortion of the nearestneighbor bonds between Fe atoms and between Fe and C [235, 236, 237]. Effects on vibrational entropy from rearrangements of carbon atoms are plausible. The phonon DOS might be changed substantially by the presence of $\mathrm{C}$ in $\mathrm{Fe}$, perhaps with a stiffening of the host Fe DOS as was found in the work by Danilkin, et al., for $\mathrm{V}, \mathrm{Nb}$, and $\mathrm{Ta}$ with interstitial atoms (Section 14.1). On the other hand, since $\mathrm{C}$ diffuses freely in bcc Fe, we should expect a substantially weaker bonding between $\mathrm{Fe}$ and $\mathrm{C}$ than between transition metals and $\mathrm{O}$, reducing any effects of stiffening. The thermodynamics of martensitic transformations has a long history of study [238, 239, 240,241, 242, 243, 244]. Nevertheless, most of the interest has been on the thermodynamics of the martensitic transformation itself, as discussed below, and not of the thermodynamics of formation of the interstitial solid solutions of $\mathrm{Fe}-\mathrm{C}$.

A structurally stable crystal must provide restoring forces against shear and dilatation strains that would change its shape. The stability of a cubic crystal 
requires these conditions on the elastic constants

$$
C_{11}>0, C_{44}>0, C_{11}^{2}>C_{12}^{2}, \frac{1}{2}\left(C_{11}-C_{12}\right)>0 .
$$

Each of these stability criteria corresponds to a different mode of deformation. For example, the last inequality, of particular interest in the study of martensite, defines the stability with respect to small amplitude shears on (110)-type planes in $\langle 110\rangle$ directions. If the ratio $\left(C_{11}-C_{12}\right)$ vanishes at a given temperature, the crystal will shear spontaneously in a "soft mode instability." The earliest explanations of martensitic transformations [114, 245] were based on soft mode instabilities. Clapp's [246] formulation used the the free energy of a crystalline solid

$$
F=F_{0}+\frac{1}{2} \sum_{i=1}^{6} \sum_{j=1}^{6} C_{i j} \eta_{i} \eta_{j}+\frac{1}{3 !} \sum_{i=1}^{6} \sum_{j=1}^{6} \sum_{k=1}^{6} C_{i j k} \eta_{i} \eta_{j} \eta_{k}+\ldots
$$

where $F_{0}$ is the free energy of the strain-free material, and Voigt notation for strains $\eta$ is used. ${ }^{43}$ Stability with respect to strain is governed by the sign of each of the six eigenvalues of the $6 \times 6$ matrix of second derivatives:

$$
\frac{\partial^{2} F}{\partial \eta_{i} \partial \eta_{j}}=F_{i j} \quad(i, j=1,6)
$$

If a particular eigenvalue vanishes at a given temperature, the material is unstable with respect to the deformation mode of the associated eigenvector. The stability analysis applied to a free energy written in terms of only the first summation in Eq. 137 will reproduce the inequalities presented in Eq. 136.

It is a general feature of the bcc structure that it is weak against longitudinal waves with $2 / 3\langle 111\rangle$ wavevectors. Phonon dispersion curves of all bcc elements show a dip in frequency of the transverse dispersion near this wavevector, or at least an anomaly. (In contrast, phonon dispersions of fcc crystals tend to increase monotonically to the edge of the Brillouin zone.) Many bcc crystals have phonon dispersions that also soften at specific frequencies along the transverse [110] branch, but the specific wavevector of these soft modes varies for different alloys. At temperatures near some martensitic transformations, these low-frequency transverse modes dip towards zero frequency, becoming unstable. This behavior could originate with a temperature-dependent change of second nearest-neighbor force constants [47], because the bcc structure is not stable with first-neighbor central forces alone. With large atom displacements, however, the interatomic force constants are not expected to behave as for small displacements, and the mechanics of how a soft mode can lead to structural changes is not yet well understood.

Many bcc metal crystals are unstable energetically at low temperatures, where a close-packed structure is preferred. In pure elements, the bcc-fcc transformation cannot cause any change in configurational entropy because the shear and shuffle processes of atom movements create the product phase deterministically. Owing to the weakness of bcc structures against various distortions, Friedel suggested some time ago that soft transverse modes stabilize the bcc structure at elevated

${ }^{43}$ Here $\eta_{1}=\epsilon_{11},=\epsilon_{22}, \eta_{3}=\epsilon_{33}, \eta_{4}=\epsilon_{12}, \eta_{5}=\epsilon_{13}$ and $\eta_{6}=\epsilon_{23}$. 
temperatures [247] (also discussed in Section 10.1). Other considerations of entropy changes during martensitic transformations and twinning in alloys were described by Cahn [248].

Murakami studied the cubic to orthorhombic martensitic transformation in $\mathrm{Au}-$ $\mathrm{Cu}-\mathrm{Zn}$ alloys [249]. Elastic constants were obtained by ultrasonic measurements, and Debye temperatures were estimated from them. The importance of vibrational entropy was demonstrated, at least qualitatively. Tong and Wayman presented similar ideas about the fcc to hcp martensitic transformation in Co-Ni [242].

Planes, Ortín, Mañosa, et al., performed a number of calorimetric studies of the heats of martensitic transformations in shape-memory alloys, which tend to have good thermodynamic reversibility. From the measured heats from $\mathrm{Cu}-\mathrm{Al}-\mathrm{Zn}, \mathrm{Cu}-\mathrm{Al}-$ $\mathrm{Ni}$, and $\mathrm{Cu}-\mathrm{Al}-\mathrm{Be}$ alloys, $\Delta H$, and the temperatures of transformation, the entropy was obtained as $\Delta S=\Delta H / T$. A typical value of $\Delta S$ was $0.2 k_{\mathrm{B}} /$ atom $[250,251$, 252]. The size of $\Delta S$ increased with the electron/atom ratio of the alloys, with $\Delta S$ varying by about $15 \%$ over the composition ranges studied. These investigators also showed that when the transformation temperature is low with respect to the Debye temperature of the alloy, the vibrational entropy of the transformation is suppressed - a consequence expected from a low occupancy of most phonon modes. Finally, they presented evidence that the martensitic transformation temperature depends on the presence of low-energy modes in the high temperature phase that help stabilize it, and the transformation is promoted when these modes are depopulated at lower temperatures.

The martensite-to-austenite transformation in NiTi has a latent heat of $(0.5 \pm$ 0.05) $k_{\mathrm{B}} /$ atom, as measured by calorimetry [253]. The phonon DOS of the two phases were obtained from inelastic scattering spectra after using a lattice dynamics simulation to correct for the different phonon scattering weights of $\mathrm{Ni}$ and $\mathrm{Ti}$. These phonon DOS curves of the austenite and martensite, Fig. 24, show substantial differences. From these data, it seems that all the transformation entropy from calorimetry is vibrational entropy. From simulations and the measured DOS, the vibrational entropy of austenite was found to be larger than that of martensite because the transverse acoustic (TA(2)) and longitudinal acoustic modes of austenite are softer than those of martensite. (Similar changes in acoustic modes of NiTi were reported previously [254].) Simulations suggest that this originates with a large and negative $1 \mathrm{NN}$ transverse force constant, which may also be responsible for the soft modes involved in the mechanism of the martensitic transformation.

The situation for martensitic transformations in Fe alloys is more complicated. Substantial changes in magnetic entropy typically accompany the martensitic transformation. For Fe-Ni alloys, the martensitic transformation is from fcc at high temperatures to approximately bcc at low temperatures. Figure 25 shows phonon DOS curves from Fe-29 at.\% Ni [255]. The phonon DOS curves were corrected for neutron weighting, using the partial DOS of ${ }^{57} \mathrm{Fe}$ atoms measured by Franz, et al., [256]. The vibrational entropy change of the martensitic transformation was $\Delta S_{\mathrm{vib}}=0.12 k_{\mathrm{B}} /$ atom, with the fcc phase having the larger vibrational entropy. It is interesting to see that the $\mathrm{Ni}$ atoms have relatively low vibrational frequencies in the bcc phase, reducing the vibrational entropy of the transformation. This $\Delta S_{\text {vib }}$ was inconsistent with the latent heats of the forward and reverse martensitic trans- 


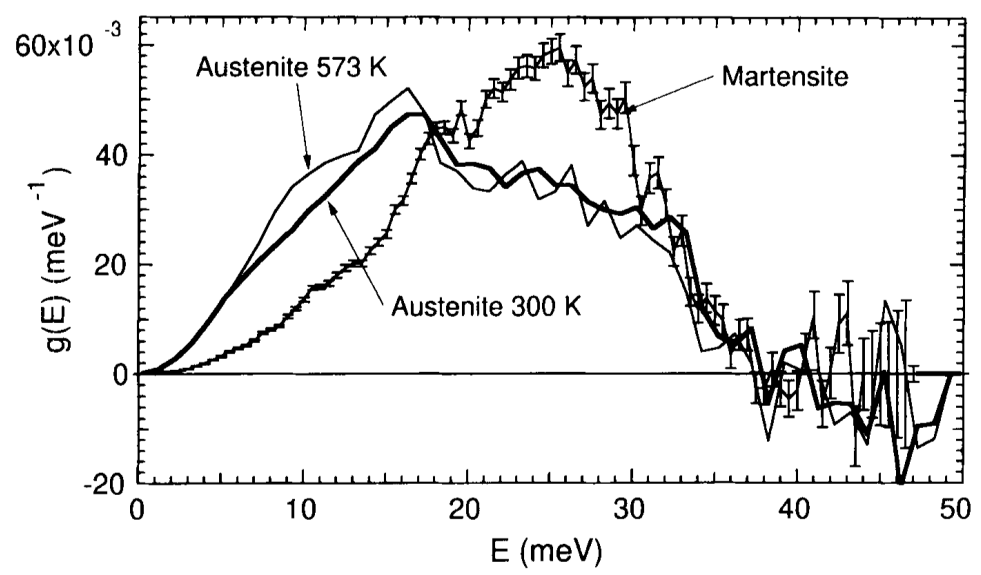

Figure 24. Phonon DOS curves of NiTi at different temperatures and different phases as labeled. The "Martensite" curve was acquired at approximately $150 \mathrm{~K}$.

formations, which were not equal. The origin of the discrepancy was the change in magnetic entropy. The martensitic transformation is hysteretic, and occurs below the Curie temperature of the bcc phase for the forward fcc $\rightarrow$ bcc transformation, and above this temperature for the reverse transformation. The change in magnetic entropy of the martensitic transformation in Fe 29 at.\% Ni proved to be about twice as large as the change in vibrational entropy.

The Heusler alloy $\mathrm{Ni}_{2} \mathrm{MnGa}$ exhibits a "magnetic shape memory" effect, where a magnetic field can change the shape of the material much as temperature changes the shape of conventional shape memory alloys like $\mathrm{Ni}$-Ti. The alloy $\mathrm{Ni}_{2} \mathrm{MnGa}$ also changes its shape by a reversible martensitic transformation, and there have been several studies of phonons in the cubic "austenite" and tetragonal "martensite" phases. Several soft phonon anomalies near the transition temperature were observed in the phonon dispersion curves, and it was suggested that they originate in part from electron-phonon interactions of a nested Fermi surface [257]. Further work on $\mathrm{Ni}_{2} \mathrm{MnGa}$ showed that the transition temperature depends on the electronic structure [258], and the thermal excitations of both magnons and phonons [259]. The magnetic and vibrational effects on the martensitic transition in $\mathrm{Ni}_{2} \mathrm{MnGa}$ seem to be comparable in importance.

\section{Microstructural Contributions}

The entropy is typically largest when the number $\Omega$ of Eq. 1 is obtained by counting entities with about one degree of freedom per atom. Consolidating $n$ degrees of freedom into one reduces $S$ by a factor of $n$, so mesoscopic and microscopic structures in materials generally have too few degrees of freedom to compete with entropic contributions from atomistic quantities. Usually it is the energy that depends on the microstructural degrees of freedom, but some exceptions are presented here. 

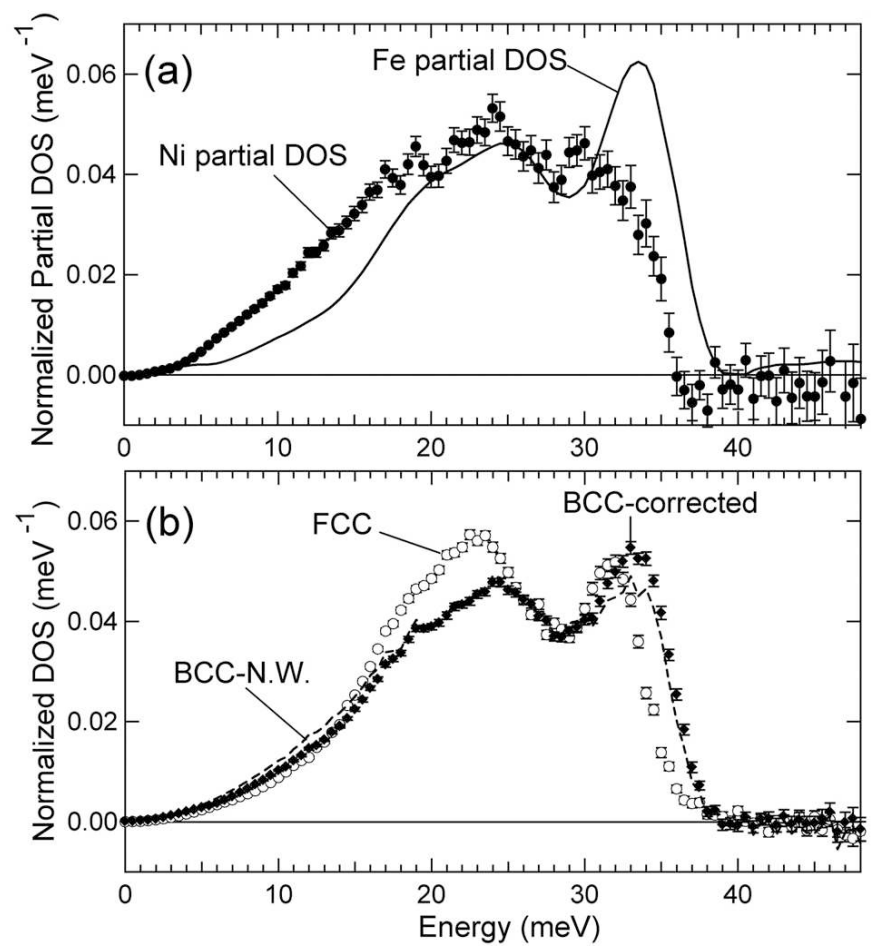

Figure 25. (a) Partial phonon DOS curves for $\mathrm{Fe}$ and $\mathrm{Ni}$ in the martensite, at room temperature. Line: Fe-pDOS obtained by Franz, et al. [256]. Dots: Ni-pDOS determined from analysis of data of $[255,256]$. (b) Room-temperature phonon DOS curves. Dashed line: neutron-weighted DOS for martensite. Diamonds: neutron-weight-corrected DOS for martensite. White circles: neutron-weighted DOS for austenite (the neutron-weight in this case is minor).

\subsection{Elastic ENERGy}

If the thermal expansion of a crystal is isotropic, all distances will expand by the same fractional length. Even an irregular packing of crystals in a polycrystalline microstructure will expand uniformly, and will not develop internal stresses with changes in temperature. On the other hand, when thermal expansion is crystallographically anisotropic, an irregular polycrystalline microstructure develops internal stresses as adjacent crystals, differing in crystallographic orientation, try to expand by different amounts along different directions. ${ }^{44}$ Here we consider the case where a stress-free polycrystalline microstructure is prepared by annealing at high temperature, but anisotropies of thermal contraction create internal stresses upon cooling.

The $\mathrm{D}_{22}$ structure is similar to a cubic fcc structure, but has a compositional modulation along (001) that gives a tetragonal distortion to the unit cell. If the $\mathrm{D0}_{22}$ structure is formed in equilibrium at high temperature and cooled, a polycrystalline microstructure will develop internal stresses at low temperature. Assuming the strains are all elastic, these internal stresses are relieved as the microstructure is heated to modest temperatures, and this stored elastic energy is converted to heat.

\footnotetext{
44 Some ceramic materials with unit cells of low symmetry may fracture under these internal stresses upon cooling.
} 


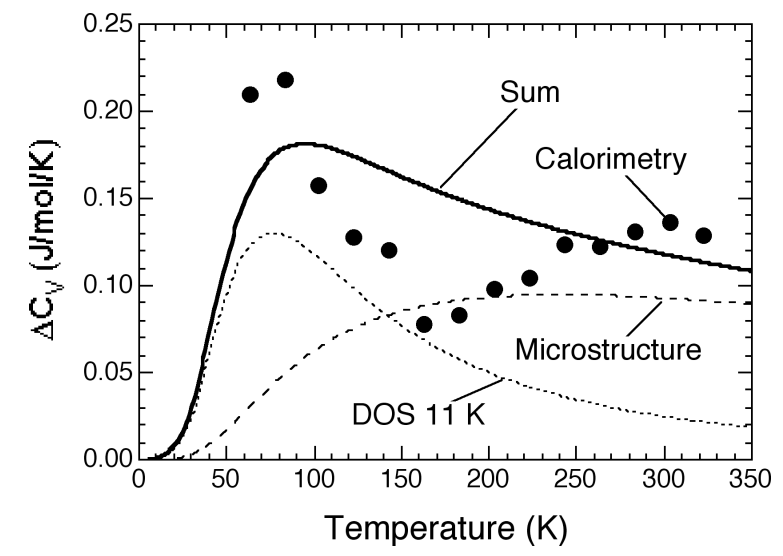

Figure 26. Dots: differential heat capacity of two samples of $\mathrm{Ni}_{3} \mathrm{~V}$. Sign is partially-disordered fcc minus $\mathrm{D0}_{22}$-ordered. After $[260,196]$.

It therefore takes less heat input to raise the temperature of this microstructure, and its heat capacity is suppressed. Evidence of this suppressed heat capacity was found for $\mathrm{D}_{22} \mathrm{Ni}_{3} \mathrm{~V}$ and $\mathrm{Pd}_{3} \mathrm{~V}$ alloys [260, 261]. In these experiments, differential scanning calorimetry was used to compare alloys in two phases: 1) cubic, prepared by quenching the sample from a high temperature in an fcc region of the phase diagram, and 2) $\mathrm{D}_{22}$ ordered, prepared by annealing in the $\mathrm{D}_{22}$ region of the phase diagram. Figure 26 shows the differential heat capacity of these two samples - the partially disordered cubic sample had the larger heat capacity. The shape of the differential heat capacity curve is quite different from the shape of Fig. 8b, however. The curve labeled "Microstructure" in Fig. 26 is the contribution obtained by assuming that the $\mathrm{D}_{22}$ ordered sample retains internal stresses at low temperature that are relieved as the solid expands. The curve labeled "DOS" in Fig. 26 was obtained from experimental phonon spectra measured for the two samples by inelastic neutron scattering. The sum of the two contributions better accounts for the measured differential heat capacity than either contribution alone.

Nevertheless, the fitted shape in Fig. 26 leaves much to be desired. The response to thermal stresses is probably more complicated than linear elastic behavior. Over a temperature range of $100 \mathrm{~K}$, differences in thermal expansion along different crystallographic directions of $10^{-5} / \mathrm{K}$ generate strains in excess of 0.001 . Over a temperature range of a few hundred $\mathrm{K}$, local plastic deformation can be induced by anisotropic thermal strains, especially at locations of stress concentrations. Plastic deformation requires mechanical work to be performed by thermal expansion, increasing the apparent heat capacity (as opposed to decreasing it by relief of internal stress). The microstructural contribution to the heat capacity can therefore change sign, and plastic deformation can cause hysteretic behavior.

The effects of elastic and plastic deformation during thermal contraction and expansion were studied in polycrystalline uranium, and its differential heat capacity was measured against a single crystal of uranium [262]. Both samples are expected to have nearly the same phonon entropy, so the study was able to isolate the microstructural effects on the heat capacity. Powder diffractometry was used to obtain internal strain distributions along different crystallographic directions. These diffrac- 
tion results were used to calculate the elastic energy in the microstructure, and this analysis of diffraction results was in reasonable agreement with the measured heat capacity. The differential heat capacity between polycrystalline and single crystal uranium changed sign with temperature as the relief of elastic stress upon heating gave way to plastic deformation.

These effects are probably not best described as part of the intrinsic thermodynamics of crystalline phases, but rather a part of the mechanics of the microstructure. They do affect bulk thermodynamic properties measurements, however, and such measurements offer a means for studying them.

\subsection{NANOSTRUCtURES}

\subsubsection{Energy and Entropy of Nanostructures}

Nanostructured materials are of widespread interest in many fields of science and engineering. A practical question is whether nanostructures are sufficiently stable at their temperatures of service, and a basic question is how the thermodynamics of nanostructured materials differs from conventional bulk materials. In short, the surfaces or interfaces of nanostructures raise the energy. On the other hand, the free energy of nanostructured materials could be reduced by sources of entropy associated with configurations of atoms or excitations in the structure, helping to stabilize the material at finite temperatures. Consider, for example, a nanostructured material with a grain boundary enthalpy of $3 \mathrm{~kJ} /$ mole $[263,264]$. At a temperature of $500 \mathrm{~K}$, an entropy of $0.75 \mathrm{k}_{\mathrm{B}}$ /atom $\left(6.2 \mathrm{~J}(\mathrm{~mol} \mathrm{~K})^{-1}\right)$ would null the free energy difference between the nanostructured material and the bulk material.

The extra configurational entropy associated with a nanocrystalline microstructure is probably not large. For consolidated nanocrystals, there can be a substantial fraction of atoms in grain boundaries, which are typically of $1 \mathrm{~nm}$ in width [264, 265]. Atoms in the grain boundary accommodate the mismatch of the bounding crystals, and cannot be positioned as arbitrarily as in a glass. Using a glass transition entropy of $\sim 1 \mathrm{k}_{\mathrm{B}}$ /atom would be a generous upper bound. This is shared over all atoms in the solid, however, so it should be reduced by the grain boundary fraction of atoms. The positions and orientations of the nanocrystals give other configurational degrees of freedom. Considering each nanocrystal as having nucleated at a point and grown to impingement with other nanocrystals, the configurations can be counted as the number of ways of distributing the nucleation points over a lattice of approximately atomic spacing. The orientational degrees of freedom can also be counted as rotations of 1-atom increments at the edge of the nanocrystal. These configurational degrees of freedom are not large for a nanocrystal of $7 \mathrm{~nm}$ dimension and $10^{5}$ atoms, when averaged over all atoms in the material. (There will be additional degrees of freedom if the positions of grain boundaries are decoupled from the centers of the nanocrystals.) Perhaps $0.1 \mathrm{k}_{\mathrm{B}} /$ atom can be obtained for the excess configurational entropy of such nanocrystals, but this seems generous. In summary, the configurational degrees of freedom of atoms become consolidated into collections of $10^{5}$ atoms, reducing the entropy by this large factor, but there are nanostructural features that bring some new degrees of freedom.

The vibrational entropy of nanostructured materials is less well understood. From the measurements available to date, the vibrational entropy difference be- 
tween nanostructured and bulk materials is larger than the configurational entropy difference, but it seems half as large as necessary to stabilize thermodynamically a nanocrystalline material at a temperature of $500 \mathrm{~K}$. Nevertheless, vibrational entropy makes a difference for the stability of nanocrystalline materials. Furthermore, the vibrational dynamics of nanostructures are of interest in their own right.

\subsubsection{Low-Energy Vibrations of Nanostructures}

The eigenmodes of vibrations of small crystals have been calculated both for isolated crystals [266, 267, 268]. and for crystals with rigid constraints at their surfaces [269]. A distinguishing feature of these calculations of the DOS, later borne out by experiments, is an enhancement in the number of modes at low energies. A continuum analog for some of these low-energy modes are Rayleigh modes over the surface, which become more distinct from bulk atom motions with increasing mismatch of the acoustic impedances of the crystal and the medium in which it is embedded. These modes involve surface atoms, of course, but they also contribute to the partial DOS of atoms beneath the surface. A computer simulation of a periodic array of small nanocrystals with Lennard-Jones interatomic potentials also showed an enhancement of the low-energy modes of the phonon DOS [270]. The calculated DOS was similar to that of a glass, but curiously, the glass was more stable than the nanocrystalline microstructure of this simulation.

Experimental measurements have shown a number of differences in the phonon DOS of nanocrystalline and bulk materials. In general, the energies of the van Hove singularities are the same for materials with nanocrystals or with large crystals, indicating that the interatomic forces are the same for most atoms situated locally in regions of good crystal [271, 272, 273, 274]. This is seen in the phonon DOS curves of Fig. 27 - although the DOS is broadened with smaller crystallite size, the energies of the van Hove singularities do not shift substantially [275]. This remains true as the nanocrystalline material is subjected to pressure, as shown for bcc Fe in Fig. 28 [276]. At pressures of 0.2 and $9.5 \mathrm{GPa}$ both bulk and nanocrystalline samples were bcc, but both had transformed to hcp Fe at the highest pressure of 25-27 GPa. The energies of the van Hove singularities changed with crystal structure and with pressure, but these changes were similar for the nanocrystalline and bulk materials.

It is clear from both Figs. 27 and 28 that the phonon DOS in the nanocrystalline material has more spectral weight at energies below about $20 \mathrm{meV}$, and further analysis of the data of Fig. 27 showed that this enhancement at low energies scales inversely with the crystallite size. This enhancement of the DOS at low energies has been found in all studies of compacted nanocrystals $[272,273,274,275,277$, 278]. This trend implies that the extra spectral weight at low energies originates from degrees of freedom in the microstructure, which may include vibrations that tend to localize at grain boundary regions or propagate along them, motions of the nanocrystals with respect to each other, or some combinations of these degrees of freedom.

For consolidated nanocrystals, the enhancement in the phonon DOS at low energies does not extend to arbitrarily low energies and aritrarily long wavelengths. In measurements of phonons in the $\mu \mathrm{eV}$ energy range [279], it was found that the enhancement in the phonon DOS around $10 \mu \mathrm{eV}$ was only about half of what was found around $10 \mathrm{meV}$. Some enhancement of the phonon DOS is expected owing 


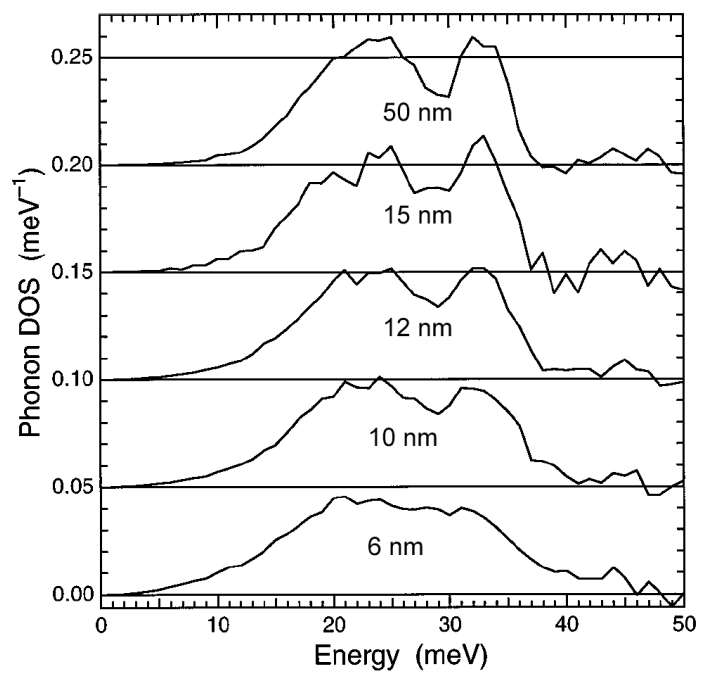

Figure 27. Phonon DOS curves from nanocrystalline fcc $\mathrm{Ni}_{3} \mathrm{Fe}$ prepared by high-energy ball milling, followed by subsequent annealing to induce grain growth to sizes as labeled. After [275].

to lower average elastic constants in any composite material with elastically "soft" atom configurations at grain boundaries, and this may explain the suppression of the DOS in the $\mu \mathrm{eV}$ energy range and the reduction in bulk elastic constants. The behavior at $\mu \mathrm{eV}$ energies is therefore understood as a continuum limit, so the extra enhancement in the meV energy range is more interesting. It is likely caused by the extra degrees of freedom of the nanocrystalline microstructure. These probably originate with the spatial dimensions of the nanocrystalline features, of order $10 \mathrm{~nm}$, and these effects therefore do not extend to the continuum limit. We note in passing that this behavior of an enhancement in the DOS at low, but not arbitrarily low, energies is reminiscent of the "boson peak," of glassy materials, hinting that the boson peak may originate from nanostructured features of the glassy structure.

The enhancement of spectral weight at low energies is a widespread phenomenon, and is larger when the nanocrystalline dimensions are smaller. Further insight can be obtained by assessing whether these low energy excitations show characteristics of reduced dimensionality. In the continuum limit, all sound waves have linear dispersions with $\varepsilon / \hbar=\omega \propto k$, so in three dimensions $g(\varepsilon) \propto \varepsilon^{2}$. Especially for DOS curves measured by inelastic nuclear resonant scattering, which offers the advantages of being both incoherent and with a low background, the exponent $n$ of $g(\varepsilon) \propto \varepsilon^{n}$ can be obtained by fitting $g(\varepsilon)$ as in Fig. 28. Doing so for three DOS curves in the bcc phase at different pressures gave $n=2.00( \pm 0.015)$ for the bulk material, and $n=1.95( \pm 0.015)$ for the nanocrystalline material. Some excitations in the nanocrystalline material may have 2-dimensional character. The nanocrystals used for the data of Fig. 28 had grain sizes of $9 \mathrm{~nm}$, however, and larger effects may occur for smaller nanocrystals, or nanocrystals that are not compacted together. Lower-dimensional excitations were more prominent in a computational study where the nanocrystals were individually isolated and were very small $(2-3 \mathrm{~nm})$ [280]. In a companion experimental study, inelastic scattering from hydrogen atoms was measured, but the motions of hydrogen atoms in grain boundaries may not reflect the 


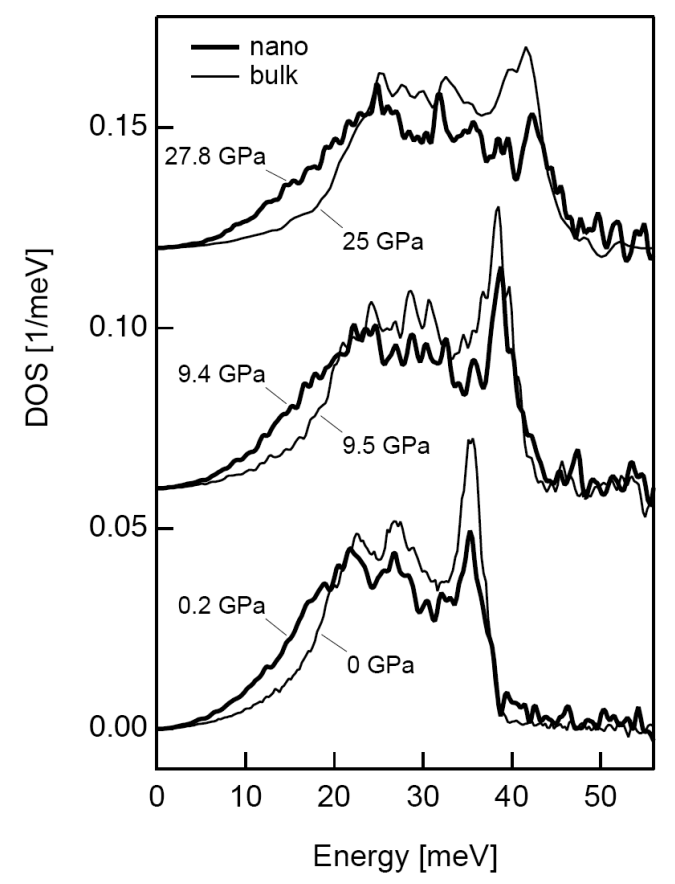

Figure 28. Phonon DOS of nanocrystalline and bulk bcc ${ }^{57} \mathrm{Fe}$ at pressures of 0 and $9.5 \mathrm{GPa}$, and hcp ${ }^{57} \mathrm{Fe}$ at approximately $26 \mathrm{GPa}$.

motions of the Pd atoms themselves [281]. A later molecular dynamics calculation on nanocrystalline microstructures of fcc $\mathrm{Ni}$ and $\mathrm{Cu}$ reported similar low-dimensional behavior for 5 and $12 \mathrm{~nm}$ nanocrystals [282].

In a challenging experiment, Roldan Cuenya measured the ${ }^{57} \mathrm{Fe}$ partial DOS of individual nanocrystals, isolated from each other, using the NRIXS method. [283]. The nanocrystals of 8-10 nm diameter consisted of bcc Fe cores, surrounded by oxide shells, and were deposited on Si wafers. At phonon energies below 5 to $10 \mathrm{meV}$, exponents $n$ in the expression $g(\varepsilon) \propto \varepsilon^{n}$ were found to be $1.33,1.84,1.86$ for three different samples, showing a clear low-dimensional behavior in the partial DOS of the Fe. This behavior depended on the shell of surface atoms of the nanocrystals, where the tendency toward 2D vibrational behavior was likely due to the large number of low-coordinated interfacial atoms. Supporting calculations showed that the lowerdimensional behavior extends from the surface to the core of the nanocrystals due to hybridization of surface and bulk modes. The evidence for lower-dimensional vibrations seems stronger in systems where there is a larger acoustic impedance mismatch between surface atoms and their surroundings, such as between isolated nanocrystals and vacuum. In such cases, however, it may be more appropriate to consider the low energy vibrations as eigenmodes of small particles [267, 268, 269].

In a separate NRIXS study, Roldan Cuenya, et al. studied phonons in thin, $8 \mathrm{~nm}$ layers of ${ }^{57} \mathrm{Fe}$ confined between layers of $\mathrm{Cr}, \mathrm{Co}, \mathrm{Cu}, \mathrm{Pd}, \mathrm{Au}$, or $\mathrm{Ag}$ [284]. There was a clear suppression of high-frequency longitudinal modes of bcc Fe when it was confined between metals having a lower cutoff of their phonon DOS. Owing to the large mismatch in phonon modes, high-frequency modes of Fe cannot propagate into the $\mathrm{Cu}, \mathrm{Pd}, \mathrm{Ag}$, and $\mathrm{Au}$ layers, and remain confined inside the thin Fe layers. Short- 
wavelength vibrational waves in the Fe layers are expected to be suppressed near the $\mathrm{Fe} / \mathrm{M}$ interfaces when $\mathrm{M}=\mathrm{Cu}, \mathrm{Pd}, \mathrm{Ag}$, and $\mathrm{Au}$, consistent with observations. The phonon DOS of the bcc Fe is shifted to lower energy, causing an increase in its vibrational entropy. In addition, thermal transport is also expected to be suppressed in these thin film multilayers.

For nanocrystals, it is generally found that phonon spectral weight is transferred to lower energies, causing an increase in the vibrational entropy as large as $0.2 k_{\mathrm{B}}$ /atom for nanocrystalline materials measured to date [273, 275, 276, 277, 285]. Nevertheless, it appears that the entropy of vibrations and configurations is only about half of what is required to give thermodynamic stabilization to a nanocrystalline microstructure at reasonable finite temperatures. Further research should show if greater stabilization is possible by the enhancement of vibrational modes at low energies. Some of this increase in vibrational entropy can be offset by the spectral weight that appears at high energies, however.

\subsubsection{Broadening of Spectral Features of Nanocrystalline Materials}

The second prominent feature of the phonon DOS of nanocrystalline materials, but one that remains less well understood, is the broadening of all features of the DOS. This broadening is at least partially independent of the transfer of spectral weight to low energies. One possible explanation is that the broadening originates with the "damping" of the phonons, which means the rapid transfer of energy from one phonon state to another, likely caused by an anharmonic perturbation. Such behavior can originate with anharmonic interatomic potentials, as may perhaps exist for atoms in irregular sites in grain boundaries. These broadenings can be large, as shown in Fig. 27. If the effects originate with damping, the spectral shape of a damped harmonic oscillator may be appropriate

$$
D_{\varepsilon^{\prime}}(\varepsilon)=\frac{1}{\pi \mathcal{Q} \varepsilon^{\prime}} \frac{1}{\left(\frac{\varepsilon^{\prime}}{\varepsilon}-\frac{\varepsilon}{\varepsilon^{\prime}}\right)^{2}+\frac{1}{\mathcal{Q}^{2}}} .
$$

where $\mathcal{Q}$ is the "quality factor" of the oscillator (i.e., the number of cycles for which the fraction $\mathrm{e}^{-1}$ of the phonons will remain) and $\varepsilon$ is the range of energies around the energy of the phonon, $\varepsilon^{\prime}$. The shape of $D_{\varepsilon^{\prime}}(\varepsilon)$ is similar to a Lorentzian function when $\mathcal{Q}$ is large, but $D_{\varepsilon^{\prime}}(\varepsilon)$ goes to zero as $\varepsilon$ goes to zero. For spectra measured to date on consolidated nanocrystals, convoluting the phonon DOS of a bulk material with the function $D_{\varepsilon^{\prime}}(\varepsilon)$ has proved successful in accounting for the broadening of the phonon DOS of nanostructured materials, except for the independent effects at low energies that are caused by degrees of freedom of the nanostructure itself. Figure 29 shows such an example.

A curious feature of the broadening of the phonon DOS of nanocrystals is that sometimes it can be changed without changing the size of the nanocrystallites. As shown in Fig. 30, the phonon DOS of nanocrystalline $\mathrm{Ni}_{3} \mathrm{Fe}$ sharpens considerably after the sample is exposed to deep cryogenic temperatures, even though the low energy part of the DOS remains essentially the same. It is possible that some of the atoms in anharmonic environments, such as atoms in energetically unfavorable sites at grain boundaries, were jostled into more favorable harmonic configurations by the thermal contractions induced by cryogenic exposure [286, 287, 288, 289]. 


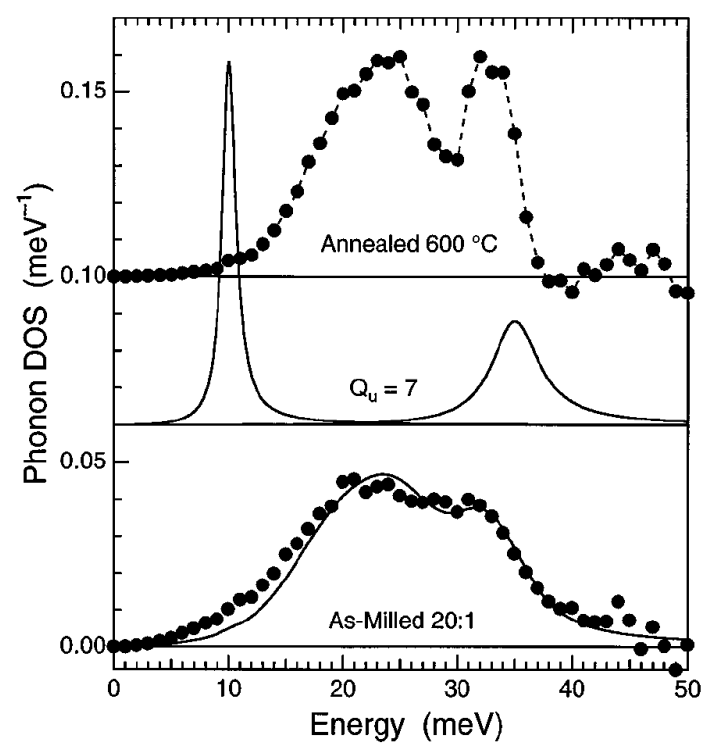

Figure 29. The characteristic shape of the damped harmonic oscillator function is shown in the center for modes at 10 and $35 \mathrm{meV}$ having the quality factor $\mathcal{Q}=7$. Solid curve at bottom is the convolution of the damped harmonic oscillator function for $\mathcal{Q}=7$ with the experimental DOS from the sample annealed at $600^{\circ} \mathrm{C}$ (points at top). Points at bottom are experimental DOS curve from $6 \mathrm{~nm}$ Ni-Fe material. After [275].

An alternative explanation for vibrational modes at high energies was suggested by D. Wolf, et al. [290]. Their molecular dynamics simulations showed modes at energies above those of the crystallites. These high-energy modes were attributed to local atomic environments in grain boundaries with reduced interatomic separations. This explanation could be consistent with the trend of Fig. 30, assuming again that cryogenic exposure or low-temperature annealing were to reduce the local atomic distortions within grain boundaries.

\section{7. f-Electron Metals}

\subsection{Cerium}

The $T$ - $P$ phase diagram of cerium metal is shown in Fig. 31. With increasing temperature, there are four solid phases at ambient pressure. Cerium is particularly unusual because its $\alpha$ and $\gamma$ phases both have the fcc structure, but their specific volumes differ by about $17 \%$ at ambient temperature. There is a solid-state critical point where the two phases merge, and it is possible to take a path in $T$ and $P$ from one phase to the other with a continuous change in lattice parameter. The fcc-fcc transformation has attracted considerable attention, in part because of suggestions that the outer electron(s) of cerium become delocalized when the $\gamma$-phase collapses into the high-density $\alpha$-phase. Nevertheless, all the phase transformations of cerium prove interesting, and we begin with the high-temperature phases.

The phonon DOS above and below the $\gamma-\delta$ (fcc-bcc) phase transformation at $999 \mathrm{~K}$ was the subject of two independent neutron scattering experiments [291, 292], 


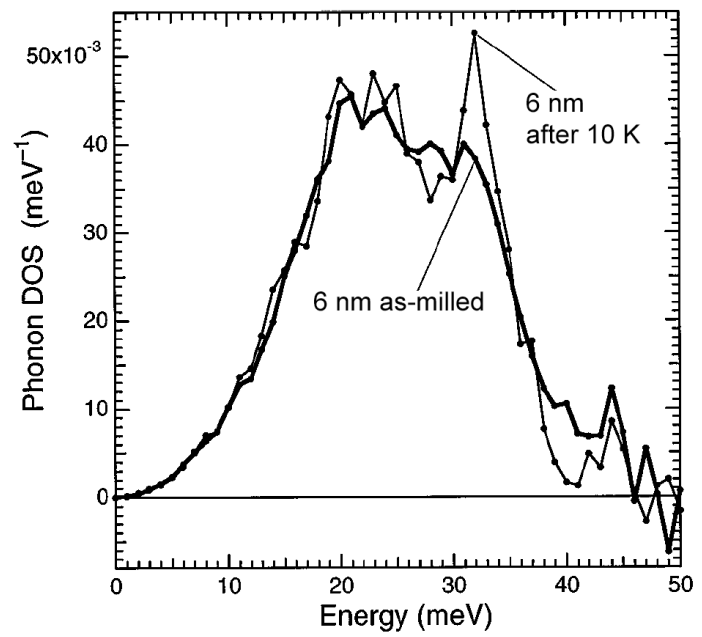

Figure 30. Phonon DOS curves at $300 \mathrm{~K}$ of fcc $\mathrm{Ni}_{3} \mathrm{Fe}$ as-milled (with $6 \mathrm{~nm}$ grain size), before and after cooling to $10 \mathrm{~K}$. After [275]. X-ray diffractometry showed no change in grain size after cryogenic exposure.

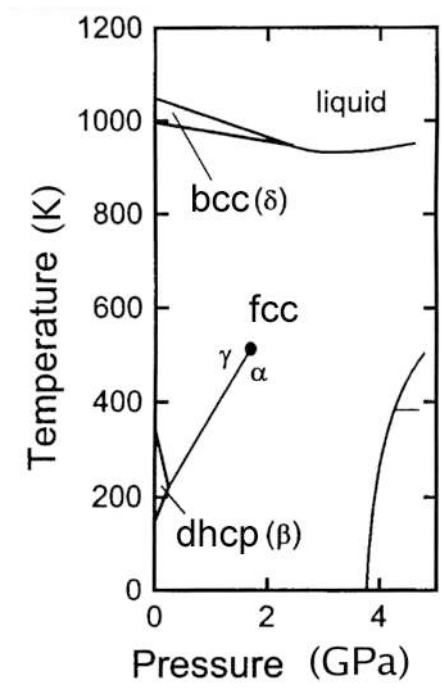

Figure 31. Low-pressure region of the cerium $T-P$ phase diagram. The solid phases at zero pressure are: fcc $\alpha$, double hcp $\beta$, fcc $\gamma$, bcc $\delta$.

using two different types of instrumentation. Cerium scatters neutrons coherently, so it is necessary to sample a wide range of momentum transfers, $Q$, to get a good representation of the full phonon spectrum. A triple-axis experiment was performed with several different values of $Q$, chosen strategically for their sampling of the phonon DOS. The conclusion from this work was that the change in vibrational entropy was too big to explain the latent heat of the $\gamma-\delta$ transformation. The second measurement with a time-of-flight instrument gave a better coverage of $Q$, and concluded that the change in vibrational entropy was even bigger. Both measurements were difficult, however, owing to the high temperature of the $\gamma-\delta$ transformation, and the large correction required for multiphonon scattering. Giving twice the weight 
to the second experiment, the vibrational entropy of the $\gamma-\delta$ transformation would be $0.64 k_{\mathrm{B}}$ /atom. The latent heat of this transformation is $0.36 k_{\mathrm{B}} /$ atom $[293,294]$, which leaves $-0.28 k_{\mathrm{B}}$ /atom to be explained by other sources of entropy. Electronic entropy is the probable cause. Such a large change in electronic entropy at such a high temperature is surprising - one might expect the Fermi-Dirac distribution and other thermal broadenings of the electronic DOS to wash out any large differences in electronic entropy.

The $\beta-\gamma$ (double hcp-fcc) phase transformation is sluggish at low temperatures. (A 20-year study showed that $283 \mathrm{~K}$ is a better estimate of the transformation temperature than that of Fig. 31 [295].) Inelastic neutron scattering experiments performed on the $\beta$ and $\gamma$ phases of cerium $[296,297]$ found only a small difference in vibrational entropy, approximately $0.09 k_{\mathrm{B}}$ /atom at the transformation temperature. The vibrational entropy was the largest contributor to the small latent heat at the transformation temperature, but other sources of entropy were much larger at lower temperatures. In particular, the entropy difference between the $\gamma$ and $\beta$ phases had large contributions from the difference in heat capacity of the crystal field levels of the two phases, which was as large as $-0.4 k_{\mathrm{B}}$ /atom at approximately $40 \mathrm{~K}$. The difference in electronic entropy of the $\gamma$ and $\beta$ phases made a small, negative contribution at all temperatures. The spin fluctuations are responsible for significant entropy, but curiously, the spin fluctuations in both phases are rather similar, and do not contribute a significant entropy to the phase transformation.

The celebrated $\alpha-\gamma$ (fcc-fcc) phase transformation in pure Ce is usually induced by pressure, but it is possible to obtain this phase transformation as a function of temperature in the alloy $\mathrm{Ce}_{0.9} \mathrm{Th}_{0.1}$. An inelastic neutron scattering experiment reported differences in the phonon DOS of the $\alpha$ - and $\gamma$-phases, but these were responsible for only a negligible difference in the vibrational entropy of the two phases [298]. The entropy of the $\alpha-\gamma$ transformation in $\mathrm{Ce}_{0.9} \mathrm{Th}_{0.1}$ originates almost entirely from the entropies of electrons and spins. These contributions are shown in Fig. 32. The electronic entropy from disorder in the electron occupancies near the Fermi surface makes a modest contribution to the difference in entropies of the two phases. The ground state spin fluctuation entropy was deduced from the quasielastic magnetic scattering, and the crystal field entropy from the inelastic excitation of a broad crystal field transition at $16 \mathrm{meV}$. These magnetic contributions are very large, and are the main source of the entropy difference between the two fcc phases of $\mathrm{Ce}_{0.9} \mathrm{Th}_{0.1}$.

The $\alpha-\gamma$ transformation in pure Ce seems to have a large change in vibrational entropy, unlike the $\alpha-\gamma$ transformation in $\mathrm{Ce}_{0.9} \mathrm{Th}_{0.1}$. Jeong, et al., performed diffraction measurements on pure Ce metal, crossing the $\alpha-\gamma$ phase boundary by varying $T$ at fixed $P$, and by varying $P$ at fixed $T$ [300]. They observed a discontinuity in the Debye-Waller factor at the phase transformation, indicating that the vibrational entropy of $\gamma$-Ce is $0.75 \pm 0.15 \mathrm{k}_{\mathrm{B}}$ /atom larger than that of $\alpha$-Ce. The mean-squared displacements of atoms in a phonon of frequency $\omega$ go as $\omega^{-2}$, so the Debye-Waller factor emphasizes low-frequency phonons more strongly than the $-\ln \omega$ for the vibrational entropy of Eq. 16. This difference could be a problem if the shape of the phonon spectrum differs between different phases, as is the case for $\mathrm{Ce}_{0.9} \mathrm{Th}_{0.1}$. Nevertheless, Jeong, et al., performed diffraction measurements on this alloy, too, 


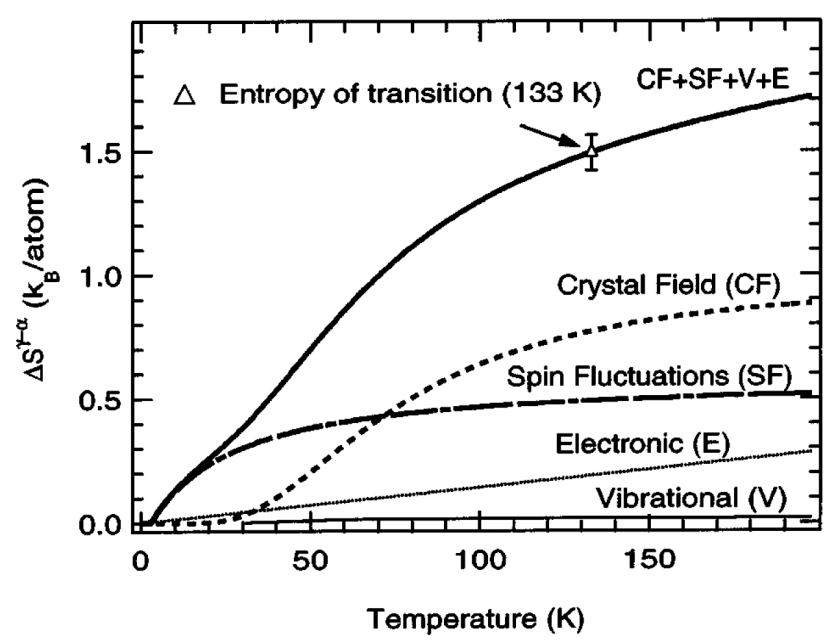

Figure 32. Contributions to the entropy difference between the $\gamma$ and $\alpha$ phase of $\mathrm{Ce}_{0.9} \mathrm{Th}_{0.1}$. The thick curves is the sum of the four components. The electronic component was adjusted so that the sum equaled the entropy measured by [299].

and found small changes in the Debye-Waller factor across the phase transformation [301], qualitatively consistent with prior neutron scattering results. It seems that pure Ce has a large vibrational entropy of its $\alpha^{-}-\gamma$ transformation (accounting for about half the transformation entropy), but the addition of $10 \% \mathrm{Th}$ suppresses the vibrational entropy of the $\alpha-\gamma$ transformation.

\subsection{Uranium and Plutonium}

Somewhat like cerium, uranium assumes 3 solid phases at different temperatures and ambient pressure. At low temperatures, the orthorhombic $\alpha$-phase shows a number of unusual properties such as a charge-density wave transition [302, 303]. Such an instability in electronic structure suggests that the atom displacements in phonons may alter the electronic structure, and the interatomic forces and phonon frequencies may change with temperature. A temperature-dependent electronic effect on phonons was in fact found in a neutron inelastic scattering study on $\alpha-\mathrm{U}$ [304]. The quasiharmonic phonon softening in $\alpha$-uranium expected with Eq. 83 and the approach of Section 6.3 is small, owing to the low bulk modulus. The actual phonon softening to a temperature of $940 \mathrm{~K}$ gives an increase of vibrational entropy of $1.5 k_{\mathrm{B}}$ /atom, approximately a factor of 10 larger than the quasiharmonic prediction. Typical anharmonic potentials cannot account for such a large softening of the phonons. The clear implication is that the interatomic forces are weakening with temperature. It is not known if this originates with thermal excitations of electron energy levels, electron energy level broadenings caused by shortened electron lifetimes, or by changes in electronic levels with temperature that are caused by larger atomic mean-squared displacements, for example.

At ambient pressure, plutonium has even more solid phases, six, at different temperatures, having atomic volumes differing by as much as $20 \%$. With such differences in local atomic distances, the $5 f$ bands of $\mathrm{Pu}$ differ considerably in their widths for 
the different phases. For the denser phases, the Peierls distortions of the $5 f$ bands are responsible for the formation of crystal structures of low symmetry $[305,306]$. The fcc $\delta$-phase of $\mathrm{Pu}$ is intermediate in its $5 f$ localization, and temperature causes some odd behavior, such as negative thermal expansion. The fcc $\delta$-phase can be stabilized over a broad range of temperature by alloying with 2 at.\% Ga or 5 at. $\% \mathrm{Al}$ (although the thermal expansion becomes positive for the latter). Thermal neutron scattering from $\mathrm{Pu}$ is possible for samples with a high enrichment of the ${ }^{242} \mathrm{Pu}$ isotope, which has an acceptable absorption cross section. It is also possible to measure phonon dispersions with inelastic x-ray scattering, which does not require isotopic control, and a full set of phonon dispersions were measured on a small single crystal of $\mathrm{Pu}-$ 2 at.\% Ga at ambient temperature [307]. An anomaly in the low transverse [011] phonon branch, and small anisotropic elastic constants were attributed to electronphonon interactions. Measurements of the phonon DOS at temperatures of $300 \mathrm{~K}$ and below were performed on $35 \mathrm{~g}$ of ${ }^{242} \mathrm{Pu}-5$ at. $\% \mathrm{Al}$ [308]. There was generally good agreement with the results of [307], but from 27 to $300 \mathrm{~K}$ there was a large overall softening and broadening of the phonon spectrum. Similar to the case of U, for $\mathrm{Pu}$ the quasiharmonic softening expected from Section 6.3 was more than a factor of ten too small to account for the phonon softening - the softening occurs with relatively little change in lattice parameter. Such behavior seems plausible because temperature should have large effects on electrons in very narrow $5 f$ bands, but detailed predictions of electron-phonon interactions in $\mathrm{Pu}$ are only now becoming available [309].

The low temperature phases of $\mathrm{Pu}$ tend to have smaller specific volumes than the high temperature phases, and the actinide metals to the right of $\mathrm{Pu}(\mathrm{Am}, \mathrm{Cm}$, Bk...) tend to be much larger in specific volume. It has been suggested that for the larger phases of $\mathrm{Pu}$, and for the heavier actinides, the $5 f$ electrons become localized and non-bonding [310]. With these changes we may expect lower phonon frequencies. Wallace used calorimetric data to show that this trend is generally true, and also showed that vibrational entropy plays a big role in stabilizing the high temperature phases [311]. The low temperature $\alpha$-phase of $\mathrm{Pu}$ was also shown to be rather anharmonic. 
Vibrational Thermodynamics of Materials

\section{Vibrational Entropy of Materials at Higher Temperatures}

\section{Interactions between Thermal Excitations of Electrons and Phonons}

\subsection{Overview of Thermal Effects on Electron and Phonon Energies}

The full Hamiltonian of the crystal, $H$, is

$$
H=H_{\mathrm{n}}+H_{\mathrm{e}}+H_{\mathrm{ep}}
$$

where $H_{\mathrm{n}}$ is the contribution from the nuclei (i.e., phonons as in Eqs. 23 or 141), $H_{\mathrm{e}}$ is the contribution from the electrons (including electron-electron interactions and thermal electronic excitations), and $H_{\mathrm{ep}}$ is the contribution from electron-phonon interactions (EPI). Figure 33 attempts to illustrate some concepts and definitions of high temperature phonon thermodynamics, especially the adiabatic EPI. Six diagrams are shown, with low temperature effects on the bottom and high temperature effects at the top. Phonon concepts for a fixed interatomic potential $\Phi(r)$ are on the left; electron concepts with a simple free electron band structure are on the right.

At low temperatures, the vibrating atoms explore a small part of their interatomic potential wells. A parabolic fit to the interatomic potential is appropriate (at least without zero-point effects), and the harmonic model follows. At higher temperatures, the "quasiharmonic" approximation is often used, where Fig. 33 shows that the parabola is altered in position and curvature to accommodate small shifts in the effective potential as the atoms vibrate about their points of equilibrium. (The thermal spread in potential energy, of order $k_{\mathrm{B}} T$, is shown by light shading.) The quasiharmonic model assumes that phonons are eigenstates of a phonon Hamiltonian for the expanded volume of the crystal, and it does not predict any shortening of phonon lifetimes. This is less appropriate at higher temperatures, where the cubic and quartic contributions to the potential energy are needed to understand the reduced phonon lifetimes, and shifts with temperature that may not depend quasiharmonically on the volume of the crystal and its bulk modulus (Section 6.3). "Anharmonic" corrections to the quasiharmonic model are needed to account for "phonon-phonon interactions" (PPI).

The electron states at right in Fig. 33 are filled to the Fermi level at $T=0$, but at higher temperatures the shading illustrates a thermal spread of energies associated with the Fermi-Dirac distribution. At higher temperatures, as the nuclei move further about their equilibrium positions, the electron band structure is altered. If this occurs without thermal excitations of electrons, the change in energy is that for anharmonic phonons, already included in $H_{\mathrm{n}}$. On the other hand, if there are atom displacements from many phonon excitations plus there are thermal electron excitations, there is a change in energy that depends on both excitations. The conditions for obtaining this extra energy are illustrated at the top of Fig. 33. This is an adiabatic electron-phonon interaction, and makes up $H_{\mathrm{ep}}$ of Eq. 140 . Although differing in its thermal factor, the adiabatic EPI has similarities to the 


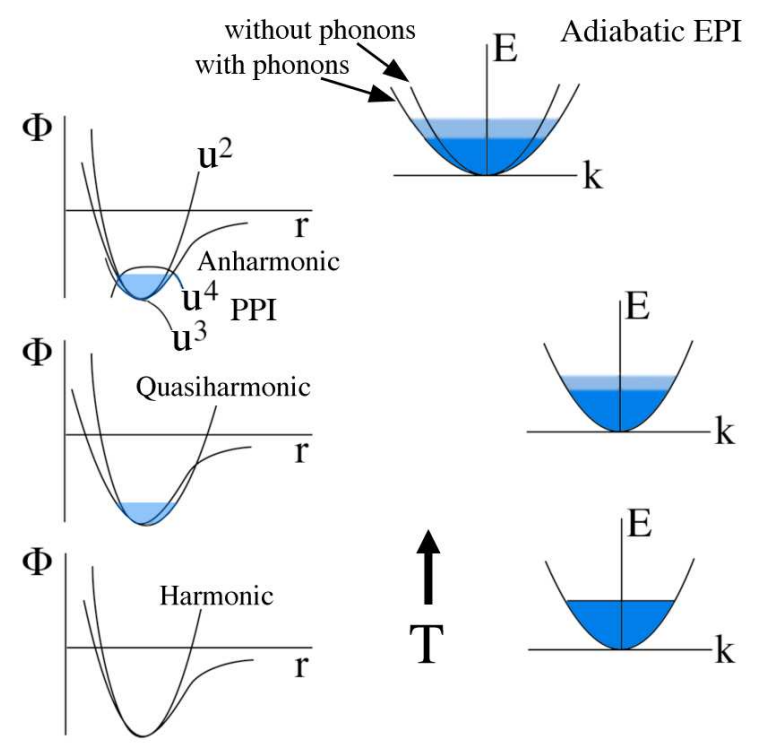

Figure 33. Effects of temperature, increasing from bottom to top, on phonons $\left(H_{\mathrm{n}}\right.$, left), electrons $\left(H_{\mathrm{e}}\right.$, right), and the existence of the adiabatic EPI when phonons alter the electronic bandstructure in the presence of electron excitations ( $H_{\mathrm{ep}}$, top). Increasing width of light shading indicates increasing thermal energy spreads.

nonadiabatic EPI responsible for Cooper pairing in conventional superconductors, and until recently it was assumed to be a weak phenomenon that could be neglected at elevated temperatures.

\subsection{Formalism of Phonon-Phonon Interactions}

To understand phonon-phonon interactions, we consider in detail the Hamiltonian for nuclear motions $H_{\mathrm{n}}$, extending the Taylor series of Eq. 23

$$
\begin{aligned}
H_{\mathrm{n}}=\Phi_{0} & +\sum_{\kappa} \frac{\vec{p}_{\kappa}^{2}}{2 m}+\frac{1}{2 !} \sum_{\kappa \alpha} \sum_{\kappa^{\prime} \alpha^{\prime}} \Phi_{\alpha \alpha^{\prime} \kappa \kappa^{\prime}} u_{\alpha \kappa} u_{\alpha^{\prime} \kappa^{\prime}} \\
& +\frac{1}{3 !} \sum_{\kappa \alpha} \sum_{\kappa^{\prime} \alpha^{\prime}} \sum_{\kappa^{\prime \prime} \alpha^{\prime \prime}} \Phi_{\alpha \alpha^{\prime} \alpha^{\prime \prime} \kappa \kappa^{\prime} \kappa^{\prime \prime}} u_{\alpha \kappa} u_{\alpha^{\prime} \kappa^{\prime}} u_{\alpha^{\prime \prime} \kappa^{\prime \prime}} \\
& +\frac{1}{4 !} \sum_{\kappa \alpha} \sum_{\kappa^{\prime} \alpha^{\prime}} \sum_{\kappa^{\prime \prime} \alpha^{\prime \prime}} \sum_{\kappa^{\prime \prime \prime} \alpha^{\prime \prime \prime}} \Phi_{\alpha \alpha^{\prime} \alpha^{\prime \prime} \alpha^{\prime \prime \prime} \kappa \kappa^{\prime} \kappa^{\prime \prime} \kappa^{\prime \prime \prime}} u_{\alpha \kappa} u_{\alpha^{\prime} \kappa^{\prime}} u_{\alpha^{\prime \prime} \kappa^{\prime \prime}} u_{\alpha^{\prime \prime \prime} \kappa^{\prime \prime \prime}}+\ldots,
\end{aligned}
$$

where we have simplified the development to one atom per unit cell, so $u_{\alpha \kappa}$ is the displacement of atom $\kappa$ along Cartesian direction $\alpha$.

The transformation to normal coordinates for an infinite periodic crystal is

$$
\vec{U}_{\vec{k}_{i}}=\frac{1}{\sqrt{N m}} \sum_{\vec{r}_{\kappa}} \vec{u}_{\vec{r}_{\kappa}} \mathrm{e}^{-\mathrm{i} \vec{k}_{i} \cdot \vec{r}_{\kappa}}
$$

and by Fourier inversion

$$
\vec{u}_{\vec{r}_{\kappa}}=\frac{1}{\sqrt{N m}} \sum_{\vec{k}_{i}} \vec{U}_{\vec{k}_{i}} \mathrm{e}^{+\mathrm{i} \vec{k}_{i} \cdot \vec{r}_{\kappa}} .
$$


Substituting Eq. 143 into Eq. 141, we identify the Fourier transform of $\Phi_{\alpha \alpha^{\prime} \kappa \kappa^{\prime}}$, and use it to define the dynamical matrix of Eqs. 30 and 31. Our interest now is in the higher-order terms of Eq. 141, which cause anharmonic behavior when the atoms are vibrating with substantial amplitude. Using Eqs. 141 - 143 we can assess phonon-phonon interactions with a classical approach, which is valid at high temperatures.

There is, however, a clarity to be gained in working with the quantized phonon field (a displacement field in the crystal), especially when considering excitations of nonlocal electron states simultaneously with phonon excitations. The phonon field operator is $A_{\vec{k}_{i}}$

$$
A_{\vec{k}_{i}}=a_{\vec{k}_{i}}+a_{-\vec{k}_{i}}^{\dagger}=A_{-\vec{k}_{i}}^{\dagger} .
$$

The raising and lowering operators $a_{\vec{k}_{i}}^{\dagger}$ and $a_{\vec{k}_{i}}$ create and annihilate the phonon $\vec{k}_{i}$. They are constructed from the momentum and position operators of the Hamiltonian when a plane wave solution is considered for one $\vec{k}_{i}$. The reader should have references showing the properties of $a^{\dagger}$ and $a$ for a simple harmonic oscillator [312], and the $a_{\vec{k}_{i}}^{\dagger}$ and $a_{\vec{k}_{i}}$ work the same way for a single normal mode of a harmonic crystal. The formalism is now in the "occupation number representation," where $n$ phonons of wavevector $\vec{k}_{i}$ are created with $n$ raising operations as $\left(a_{\vec{k}}^{\dagger}\right)^{n}|0\rangle_{\vec{k}}=(n !)^{-1 / 2}|n\rangle_{\vec{k}}$. The phonon field operators are related to the displacements (now operators) as Fourier coefficients

$$
\vec{u}\left(\vec{r}_{\kappa}\right)=\sum_{\vec{k}_{i}} \sqrt{\frac{\hbar}{2 N m \omega_{\vec{k}_{i}}}} \vec{e}\left(\vec{k}_{i}\right) \mathrm{e}^{\mathrm{i} \vec{k}_{i} \cdot \vec{r}_{\kappa}} A_{\vec{k}_{i}} .
$$

Note the similar roles of the phonon field operator $A_{\vec{k}_{i}}$ in Eq. 145 and the vector $\vec{U}_{\vec{k}_{i}}$ in Eq. 143.

By substituting Eq. 145 into Eq. 141, the dynamical matrix is recovered from the quadratic term, with prefactors such as $\hbar /\left(4 \omega_{\vec{k}_{i}} \omega_{\vec{k}_{j}}\right)$. We define similarly the transformed higher-order terms, so Eq. 141 becomes

$$
\begin{aligned}
\hat{H}_{\mathrm{n}} & =\Phi_{0}+\sum_{\vec{k}_{i}} \hbar \omega_{\vec{k}_{i}}\left(a_{\vec{k}_{i}}^{\dagger} a_{\vec{k}_{i}}+\frac{1}{2}\right) \\
& +\sum_{\vec{k}_{i}} \sum_{\vec{k}_{j}} \sum_{\vec{k}_{k}} V\left(\vec{k}_{i}, \vec{k}_{j}, \vec{k}_{k}\right) A_{\vec{k}_{i}} A_{\vec{k}_{j}} A_{\vec{k}_{k}} \\
& +\sum_{\vec{k}_{i}} \sum_{\vec{k}_{j}} \sum_{\vec{k}_{k}} \sum_{\vec{k}_{l}} V\left(\vec{k}_{i}, \vec{k}_{j}, \vec{k}_{k}, \vec{k}_{l}\right) A_{\vec{k}_{i}} A_{\vec{k}_{j}} A_{\vec{k}_{k}} A_{\vec{k}_{l}}+\ldots,
\end{aligned}
$$

where the first sum on the right-hand side includes both the kinetic energy and the harmonic part of the potential energy (following standard development [313]), and the phonon number operator is $a_{\overrightarrow{k_{i}}}^{\dagger} a_{\overrightarrow{k_{i}}}$. The $V$ are related to the $\Phi$ as Fourier 
transforms. For example,

$$
\begin{aligned}
V\left(\vec{k}_{i}, \vec{k}_{j}, \vec{k}_{k}\right) & =\frac{1}{3 !} \sqrt{\frac{1}{N m^{3}}\left(\frac{\hbar}{2}\right)^{3} \frac{1}{\omega_{\vec{k}_{i}} \omega_{\vec{k}_{j}} \omega_{\vec{k}_{k}}}} \delta\left(\vec{k}_{i}+\vec{k}_{j}+\vec{k}_{k}-\vec{g}\right) \\
& \times \sum_{\{\alpha\}\left\{\vec{r}_{\kappa}\right\}} e_{\alpha}\left(\vec{k}_{i}\right) e_{\alpha^{\prime}}\left(\vec{k}_{j}\right) e_{\alpha^{\prime \prime}}\left(\vec{k}_{k}\right) \mathrm{e}^{+\mathrm{i}\left(\vec{k}_{i}+\vec{k}_{j}+\vec{k}_{k}\right) \cdot \vec{r}_{\kappa}} \Phi_{\alpha, \alpha^{\prime}, \alpha^{\prime \prime} \kappa, \kappa^{\prime}, \kappa^{\prime \prime}},
\end{aligned}
$$

where the factor $\delta$ signifies that the sum $\vec{k}_{i}+\vec{k}_{j}+\vec{k}_{k}$ equals a reciprocal lattice vector, $\vec{g}$. For terminology, the terms with $V\left(\vec{k}_{i}, \vec{k}_{j}, \vec{k}_{k}\right)$ and $V\left(\vec{k}_{i}, \vec{k}_{j}, \vec{k}_{k}, \vec{k}_{l}\right)$ in Eq. 146 give the energies of "phonon-phonon interactions" because they alter the phonon energies when larger vibrational displacements are present in the crystal.

Details of the phonon dispersions are immediately relevant when attempting to calculate anharmonic behavior, and several concepts have been developed in detail. For example, if the phonon dispersion relation $\varepsilon(\vec{k})$ were linear, it would be easy to add two wavevectors $\vec{k}+\vec{k}^{\prime}=\vec{k}^{\prime \prime}$, and simultaneously conserve energy $\varepsilon+\varepsilon^{\prime}=$ $\varepsilon^{\prime \prime}$. The number of three-phonon processes increases as $k^{2}$, at least for small $k$. Many phonon dispersions $\varepsilon(\vec{k})$ are concave downwards, however, so simultaneous energy and momentum conservation is often impossible unless at least one phonon lies on a different branch. Phonon processes that satisfy momentum and energy conservation depend on the symmetry of the dispersion relations, and on the crystal structure. For larger phonon wavevectors $\vec{k}$, momentum conservation is possible by adding a reciprocal lattice vector. The idea is that the momentum transferred to the entire crystal occurs with zero energy because of the large mass of the crystal. Such "umklapp" processes allow many more three-phonon interactions, but the phonon wavevectors must be of length comparable to the reciprocal lattice vector if these vector additions are possible.

An important question is how many terms are needed in Eq. 146 to account accurately for anharmonic behavior. An approximate theory of the lifetimes of optical phonons was developed with 3-phonon processes [314], and was extended to 4phonon processes to include energy shifts [315]. This approach has found application for the analysis of the thermal shifts and widths of Raman peaks, sometimes with applications to thermal conductivity (e.g. [316]). We expect the higher-order terms in Eq. 141 to be progressively smaller, but the requirement of energy conservation and the $\delta$-function for momentum conservation in Eq. 147 restricts the allowable threephonon processes, owing to restrictions imposed by the phonon dispersions $\varepsilon(\vec{k})$. Fourth-order anharmonicity can therefore play a role as significant as the third. Another complexity is that four-phonon processes can be generated from threephonon processes. The idea is that two three-phonon process such as $\vec{k}+\vec{k}^{\prime} \rightarrow \vec{k}^{\prime \prime}$ and $\vec{k}^{\prime \prime} \rightarrow \vec{k}^{\prime \prime \prime}+\vec{k}^{\prime \prime \prime \prime}$ can be combined through intermediate states $\left\{\vec{k}^{\prime \prime}\right\}$ to become a four-phonon process in second-order perturbation theory, which involves matrix elements as $\sum_{\vec{k}^{\prime \prime}}\left\langle\vec{k}^{\prime \prime \prime}, \vec{k}^{\prime \prime \prime \prime \prime}\left|H^{\prime \prime \prime}\right| \vec{k}^{\prime \prime}\right\rangle\left\langle\vec{k}^{\prime \prime}\left|H^{\prime \prime \prime}\right| \vec{k}, \vec{k}^{\prime}\right\rangle$. With effort, this field theoretic approach has been used for some physical predictions as described below. Approximate summations of the perturbation series have been performed, and some results for anharmonic heat capacity were reported for solids with Lennard-Jones potentials [318]. This approach also has conceptual value for understanding the meaning of anharmonicity. 


\subsection{Formalism of Electron-Phonon Interactions}

The large difference in energy scales of phonons and electrons can motivate the separation of the Hamiltonian of the solid into a term with nuclear coordinates for the phonons $H_{\mathrm{n}}$, and a term with electron coordinates $H_{\mathrm{e}}$ (Eq. 140). The energy of the crystal deformation caused by a phonon originates with the electrons, of course, but although this potential energy of deformation is electronic in origin, it transfers to kinetic energy in the motion of the nuclei. ${ }^{45}$ If the electrons were always in their ground states, all this energy of deformation would be associated with $H_{\mathrm{n}}$ alone (i.e., the phonons). Treating the electron system and the phonon system as two independent thermodynamic systems becomes inconsistent at finite temperature, however, because the presence of phonons alters the thermal excitations of electrons.

Very generally, the electron-phonon interaction (EPI) requires the coordinates of the electrons $\left\{\vec{r}_{\lambda}^{\mathrm{el}}\right\}$, and coordinates of the nuclei $\left\{\vec{r}_{j}^{\mathrm{n}}\right\}$

$$
H_{\mathrm{ep}}=\sum_{\lambda, j} V\left(\vec{r}_{\lambda}^{\mathrm{el}}, \vec{r}_{j}^{\mathrm{n}}\right)
$$

First assume the nuclear motions are slow enough so the electron levels adapt continuously to the evolving structure, i.e., the Born-Oppenheimer approximation. This "adiabatic approximation" does not allow the nuclear kinetic energy to alter the electron states. ${ }^{46}$ The adiabatic EPI increases with the number of phonons, but it also scales with the thermal excitations of electrons. (Effects from how the interatomic potential depends on phonon populations alone are already counted as phonon-phonon interactions.)

In this adiabatic picture, unique electron eigenstates exist for a snapshot of the nuclear thermal displacements, but these evolve continuously as the nuclei move. Taking for reference a ground state with the nuclear positions of a perfectly periodic crystal and the electronic structure at $0 \mathrm{~K}$, we seek to account for how the displaced nuclear positions alter the thermally-excited electronic energy levels (changing the electronic excitations induced by temperature). For a given electronic structure, the adiabatic electron-phonon interaction is proportional to the number of phonons, $n(\varepsilon, T)+1 / 2$, times the difference of electron occupancy with respect to the ground state, $f(T)-f(0)$, where $f(T)$ is the Fermi-Dirac distribution. When there are sharp features in the electronic DOS near the Fermi level, there will be a shift of electron chemical potential with temperature, and other temperature-dependences associated with the thermal sampling of the excited electron states.

How the electron eigenstates change with nuclear displacements is a problem that generally requires considerable detail about the states of electrons and phonons. A simple approach is to consider an electronic band formed from isotropic $s$-electrons, and a uniform dilation as may be associated with longitudinal phonons of long

\footnotetext{
45 For a harmonic phonon, the electron-phonon interaction affects equally the energies of the electrons and the phonons.

46 The "non-adiabatic" electron-phonon interaction accounts for how the electronic states are altered by the nuclear kinetic energy, not the potential energy of displaced nuclei as for the adiabatic case. The non-adiabatic EPI does not require thermal activation, and can be responsible for superconductivity.
} 
wavelength,

$$
H_{\mathrm{ep}}^{\mathrm{D}}=-\sum_{\lambda} D \Delta\left(\vec{r}_{\lambda}^{\mathrm{el}}\right) .
$$

where $\Delta\left(\vec{r}_{\lambda}^{\mathrm{el}}\right)$ is the fractional change in volume at $\vec{r}_{\lambda}^{\mathrm{el}}$, and $D$ is a "deformation potential," typically a few $\mathrm{eV} .{ }^{47}$ This simple approach is convenient because the $\Delta\left(\vec{r}_{\lambda}^{\mathrm{el}}\right)$ is related to the displacement $\vec{u}$ as through its divergence: $\Delta\left(\vec{r}_{\lambda}^{\mathrm{el}}\right)=\vec{\nabla} \cdot \vec{u}$, so

$$
H_{\mathrm{ep}}^{\mathrm{D}}=-D \sum_{\lambda} \vec{\nabla} \cdot \vec{u}\left(\vec{r}_{\lambda}^{\mathrm{el}}\right)
$$

For longitudinal phonons

$$
\vec{e}\left(\vec{k}_{i}\right)=\frac{\vec{k}_{i}}{k_{i}},
$$

and by taking the divergence of Eq. 145 we have, now in the occupation number representation

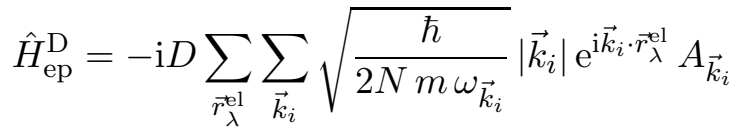

Consistent with the second quantization formalism for phonons, fermion field operators are used for the electrons

$$
\Psi^{\dagger}\left(\vec{r}_{\lambda}^{\mathrm{el}}\right)=\sum_{\vec{k}_{\lambda}} C_{\vec{k}_{\lambda}}^{\dagger} \mathrm{e}^{-\mathrm{i} \vec{k}_{\lambda} \cdot \vec{r}_{\lambda}^{\mathrm{el}}}
$$

which when applied to a multi-electron state, places an electron in the state $\vec{k}_{\lambda}$ (the operator $C_{\vec{k}_{\lambda}}$ removes an electron from this state). When small, $\hat{H}_{\mathrm{ep}}^{\mathrm{D}}$ in Eq. 152 is a perturbation that mixes electronic states as $\left\langle\Psi_{f}\left|\hat{H}_{\mathrm{ep}}^{\mathrm{D}}\right| \Psi_{i}\right\rangle$. This integral can be evaluated by using the fermion field operators $C$ and $C^{\dagger}$, where the exponential phase factors cancel the $k$-space integration unless there is a conservation of wavevector. This forces the same total $\vec{k}_{\lambda}+\vec{k}_{i}$ for the creation operators as for the annihilation operators

$$
\hat{H}_{\mathrm{ep}}^{\mathrm{D}}=-\mathrm{i} D \sqrt{\frac{\hbar}{2 N m}} \sum_{\vec{k}_{\lambda}} \sum_{\vec{k}_{i}}^{\text {electron phonon }} \sqrt{\frac{k_{i}}{c_{L}}} A_{\vec{k}_{i}} C_{\vec{k}_{\lambda}+\vec{k}_{i}}^{\dagger} C_{\vec{k}_{\lambda}},
$$

plus an analogous term with $A_{\vec{k}_{i}}^{\dagger} C_{\vec{k}_{\lambda}+\vec{k}_{i}} C_{\vec{k}_{\lambda}}^{\dagger}$ for phonon creation. For Eq. 154 we also used a linear dispersion relationship $\omega_{L}\left(k_{i}\right)=c_{L} k_{i}$, appropriate for long-wavelength, longitudinal acoustic phonons. In accounting for the effects of phonons on the electron states, it is usually necessary to consider a more general case than the isotropic deformation potential of Eq. 149 as

$$
I \propto\left\langle\vec{k}_{\lambda}+\vec{k}_{\lambda}\left|\vec{e}_{j}\left(\vec{k}_{j}\right) \cdot \nabla_{\vec{r}_{j}^{\mathrm{n}}} V\left(\vec{r}_{\lambda}^{\mathrm{el}}, \vec{r}_{j}^{\mathrm{n}}\right)\right| \vec{k}_{\lambda}\right\rangle
$$

\footnotetext{
47 It is not typical to have electron energies shifted by several eV under high compressions, so the $D$ typically pertain to small elastic distortions over a finite volume of crystal.
} 
where the gradient is with respect to the nuclear coordinates. ${ }^{48}$

In general, there are two lower order terms that are used to describe the electronphonon interaction. The first is a generalization of the previous result Eq. 154

$$
\hat{H}_{\mathrm{ep}}^{1}=\sum_{\vec{k}_{i}} \sum_{\vec{k}_{\lambda}} V^{\mathrm{ep}}\left(\vec{k}_{i}, \vec{k}_{\lambda}\right) A_{\vec{k}_{i}} C_{\vec{k}_{\lambda}+\vec{k}_{i}}^{\dagger} C_{\vec{k}_{\lambda}}
$$

It accounts for the processes where an electron is excited from state $\vec{k}_{\lambda}$ to $\vec{k}_{\lambda}+$ $\vec{k}_{i}$, simultaneously with the annihilation of a phonon in state $\vec{k}_{i}$, or creation of a phonon in state $-\vec{k}_{i}$. Momentum conservation restricts significantly these threephonon processes. The second low-order term for electron-phonon coupling is $\hat{H}_{\mathrm{ep}}^{2}$, and it includes a factor from second-order perturbation theory, $\sum_{\vec{k}^{\prime}}\left\langle\vec{k}|\Omega| \vec{k}^{\prime}\right\rangle\left\langle\vec{k}^{\prime}|\Omega| \vec{k}\right\rangle$. Note that the initial and final states of the electron have the same $\vec{k}$, so there is no change of electron state by this process. Nevertheless, the scattering into the virtual states $\left\{\vec{k}^{\prime}\right\}$ serve to alter the self-energy of the electron.

For calculating the thermodynamic effects of electron-phonon coupling, it is more useful to know how the electron-phonon coupling depends on energy, rather than momentum as in Eq. 156 for $\hat{H}_{\mathrm{ep}}^{1}$. This requires averaging over all electron states near the Fermi surface separated by $\vec{k}_{j}$ and differing in energy by a selected $\hbar \omega$. Most work on electron-phonon coupling has focused on superconductivity, where $\hbar \omega$ is rather small, and the electron states are close enough to the Fermi surface that it is reasonable to use ground state Fermi surface properties. The Éliashberg coupling function $\alpha^{2} g(\omega)$, where $g(\omega)$ is the phonon DOS, accounts for all scattering between electron states on the Fermi surface. The function $\alpha^{2} g(\omega)$ often looks similar $g(\omega)$, although some parts of the spectrum are given different weights. An important moment is calculated by weighting by $\omega^{-1}$, and can be shown to be

$$
2 \int_{0}^{\omega_{\max }} \frac{\alpha^{2} g(\omega)}{\omega} \mathrm{d} \omega=\frac{N\left(E_{\mathrm{F}}\right)\left\langle I^{2}\right\rangle}{M\left\langle\omega^{2}\right\rangle}=\lambda
$$

where $\left\langle I^{2}\right\rangle$ is an average of $I^{*} I$ of Eq. 155 (or its analog) over all directions, $\left\langle\omega^{2}\right\rangle$ is a second moment of the phonon DOS, $N\left(E_{\mathrm{F}}\right)$ is the electron DOS at the Fermi surface, and $\lambda$ is the "electron-phonon coupling parameter." The strength of the electron-phonon coupling scales with $\lambda$.

\section{Phonon-Phonon Anharmonicity}

\subsection{Damped Harmonic Oscillator}

The equation of motion for a classical damped harmonic oscillator is

$$
m \frac{\mathrm{d}^{2} x}{\mathrm{~d} t^{2}}+b \frac{\mathrm{d} x}{\mathrm{~d} t}-k x=0 .
$$

\footnotetext{
48 "Frölich coupling" is another example of an EPI, where longitudinal optical phonons create an electric field in an ionic solid. In this case, the Fourier transform of a Coulomb potential gives a factor of $4 \pi k_{i}^{-2}$, and with two ions involved, $\hat{H}_{\mathrm{ep}}^{\mathrm{Fr}}$ goes as $k_{i}^{-1}$ (instead of the $k_{i}^{+1 / 2}$ for $\hat{H}_{\mathrm{ep}}^{\mathrm{D}}$ in Eq. 154). Transition rates, or scattering rates, go as the square of these quantities.
} 
We define the dimensionless quality factor $\mathcal{Q}$ as

$$
\mathcal{Q} \equiv \frac{\sqrt{k m}}{b}
$$

A solution of Eq. 158 is

$$
x(t)=X_{0} \mathrm{e}^{-\left[\mathrm{i}+(2 \mathcal{Q})^{-1}\right] \omega t},
$$

where the frequency $\omega$ differs somewhat from that of the undamped harmonic oscillator (for which $\omega_{0}=\sqrt{k / m}$ )

$$
\omega=\omega_{0} \sqrt{\frac{1}{1+(2 \mathcal{Q})^{-2}}} .
$$

When $\mathcal{Q}$ is large, the frequency shift $\Delta$ is approximately

$$
\Delta=-\frac{\omega_{0}}{2}\left(\frac{1}{2 \mathcal{Q}}\right)^{2} \text {. }
$$

The energy spectrum of the damped harmonic oscillator was given in Eq. 139 for $\varepsilon^{\prime}=\hbar \omega$. When $\mathcal{Q}$ is large, Eq. 139 has the form of a Lorentzian function with half-width-at-half-maximum, $\Gamma$, of

$$
\Gamma=\frac{\omega_{0}}{2 \mathcal{Q}} .
$$

For a damped classical harmonic oscillator, the ratio of peak broadening to peak shift is

$$
\frac{\Gamma}{\Delta}=-4 \mathcal{Q}
$$

Equation 164 is convenient for testing against experimental measurements.

When the vibrational entropy is evaluated with Eq. 14, there is a change to $S_{\text {vib }}$ when a DOS curve $g(\varepsilon)$ is broadened by the damped harmonic oscillator power spectrum of Eq. 139. The shape of the damped oscillator spectrum is asymmetrical, going to zero at $\varepsilon=0$, and having more weight on its higher energy side. At high temperatures, where the phonon occupancy is large, the broadening serves to reduce the vibrational entropy. ${ }^{49}$ (There is a compensating effect from the lowering of the frequencies by damping, but this effect is smaller.)

The damping of a classical harmonic oscillator is understood as a dissipative process that transforms the energy of a macroscopic system into heat. Phonons are the heat, however, so the damping of a phonon cannot be understood in the same way. For example, adding a dissipative term to the Schrödinger equation would dissipate probability, not energy. It is necessary to treat phonon lifetimes as the transfer of energy between phonon modes, so a multi-body quantum mechanics is immediately necessary. The coupling of anharmonic quantum oscillators allows probability to transfer between the different oscillators, sometimes with energy shared

\footnotetext{
49 When two DOS curves such as $g^{\alpha}(\varepsilon)$ and $g^{\beta}(\varepsilon)$ of Eq. 16 are both broadened by damped oscillator functions of the same $\mathcal{Q}$, there is no effect on $\Delta S_{\mathrm{vib}}^{\beta-\alpha}$.
} 
by more than one. Such a model is convenient because it conserves energy, and a Hamiltonian can be written for the problem. If only a few oscillators are involved, however, a state will recur with some characteristic time. This may or may not be an issue for understanding experimental measurements of phonon lifetimes, but it will lead to confusion about the meaning of dissipation and irreversibility. The solution to the problem is to have a dense distribution of oscillators in the heat bath, which is an appropriate model for a macroscopic system with many degrees of freedom. For this reason, the damping of a quantum oscillator is sometimes analyzed as the imaginary part of the dynamical susceptibility of an external field interacting with the oscillator [319].

Self-consistent phonon theories offer an alternative to the perturbation theory approach described in Section 19.2. In essence, these methods make a modification to the dynamical matrix of harmonic theory. Horton and Cowley develop a simple one-dimensional example where the force constant, $\mathrm{d}^{2} V / \mathrm{d} x^{2}$, is convoluted with a Gaussian function in $x$ [320]. A set of equations is solved for consistency between the breadth of this Gaussian and the phonon frequency. This first-order approach uses a symmetrical interatomic potential without odd terms in $x$. This is also true for the more general first-order self-consistent phonon theory developed by Wallace, who then demonstrates interactions between the first-order phonons from the cubic terms in the potential [46].

\subsection{Perturbation Theory}

The usual approach to calculating how the frequencies and lifetimes of phonons change with temperature is with perturbation theory, using harmonic phonons as a basis set, and the last two terms of Eq. 146 as perturbations that alter the energies of the vibrational states. The phonon lifetime is calculated from Fermi's second golden rule, where transition rates are calculated individually by expanding the operators $A_{\vec{k}_{i}}$ in Eq. 146, and using these to couple the initial and final states of harmonic or quasiharmonic phonons.

Perturbation theory is performed for the atoms about their equilibrium configuration at elevated temperature, i.e., the thermal expansion consistent with quasiharmonic theory is already taken into account. For centro-symmetric crystals, the cubic terms in Eq. 141 with $\Phi_{\alpha \alpha^{\prime} \alpha^{\prime \prime} \kappa \kappa^{\prime} \kappa^{\prime \prime}} u_{\alpha \kappa} u_{\alpha^{\prime} \kappa^{\prime}} u_{\alpha^{\prime \prime} \kappa^{\prime \prime}}$ vanish because they are odd in the atom displacements, $u$. For example, the cubic energy contribution from the positive displacement of an atom with respect to its neighbor on the right is exactly cancelled by its negative displacement with respect to the atom at its left. For quantized vibrations, there is no energy change when $V\left(\vec{k}_{i}, \vec{k}_{j}, \vec{k}_{k}\right)$ from Eq. 146 (or $147)$ is treated as a first-order perturbation of quasiharmonic phonon states. The first non-vanishing first-order perturbation is the fourth-order term $V\left(\vec{k}_{i}, \vec{k}_{j}, \vec{k}_{k}, \vec{k}_{l}\right)$ in Eq. 146. It is physically reasonable, but argued more rigorously by Van Hove [321], that with respect to the harmonic term of relative strength 1 , the energy of the $n^{\text {th }}$ order perturbation goes as $(u / a)^{n-2}$, where $u$ is the displacement, and $a$ is the $1 \mathrm{NN}$ distance. We note that the fourth-order term in first-order perturbation theory is of relative strength $(u / a)^{2}$, but the third-order term in second-order perturbation theory is of equal strength, i.e., $(u / a)^{3-2} \times(u / a)^{3-2}=(u / a)^{2}$. The standard treatment of anharmonicity by perturbation theory includes these two parts, the quartic 


\begin{tabular}{|c|c|c|c|}
\hline Process & Terms & Creations & Annihilations \\
\hline & 1 & 3 & 0 \\
\hline & 3 & 2 & 1 \\
\hline$\longrightarrow$ & 3 & 1 & 2 \\
\hline & 1 & 0 & 3 \\
\hline
\end{tabular}

Figure 34. Summary of the eight terms from the cubic term of Eq. 146 obtained by expanding the operators $A_{\vec{k}_{i}}=a_{\vec{k}_{i}}+a_{-\vec{k}_{i}}^{\dagger}$.

term in first-order and the cubic term in second order. Many individual terms must be calculated, however, as summarized in Fig. 34 for the cubic term of Eq. 146. Not all of these terms conserve energy, such as the top and bottom processes in Fig. 34. They nevertheless do need to be considered as intermediate states (virtual states) in the second-order perturbation theory treatment of the cubic term.

Maradudin and Fein [322] obtained expressions for the phonon frequency shift in the presence of third- and fourth-order anharmonicities of Eq. 146, and these results have been discussed in a number of other references [99, 323, 324]. The phonon frequency shifts $\Delta\left(\vec{k}_{j}, \omega_{j}, T\right)$ and damping $\Gamma\left(\vec{k}_{j}, \omega_{j}, T\right)$ have the following features

- The quasiharmonic contribution to $\Delta(T)$ is identified. (It is zero if the volume of the crystal is fixed.)

- For the phonon lifetime broadening owing to scattering between quasiharmonic phonon states, the most important term is from the cubic anharmonicity. This term appears in first order perturbation theory, so it includes only those processes in Fig. 34 for which energy is conserved.

- For the shifts in phonon frequencies, the lowest-order anharmonic terms are the cubic term in second-order perturbation theory, ${ }^{50}$ and the quartic term in first-order perturbation theory. These can be comparable in strength, and comparable to the quasiharmonic part. It is not obvious what sign is expected of these anharmonic terms. They are nonzero even when there is no thermal expansion.

- At high temperatures, $\Delta(T)$ and $\Gamma(T)$ have contributions proportional to both $T$ and $T^{2}$. These usually do not reconcile with the classical prediction of Eq. 164 , in part because $\mathcal{Q}$ typically increases with temperature.

It is expected that the results for $\Delta(T)$ and $\Gamma(T)$ from perturbation theory are reliable for small atom displacements, $u / a$. The practical range of temperatures

\footnotetext{
50 In the special case when three times the phonon wavevector equals a reciprocal lattice vector, the cubic term can alter the phonon energy in first order.
} 
where these results are applicable is still a topic for research, but it is known that these results are not reliable near the melting temperature of rare-gas solids [325], and are not expected to work for quantum solids such as helium at any temperature. Unfortunately, the effort to extend these results to higher order seems very difficult, although there has been an impressive success in summing diagrams for a wellbehaved model of a rare-gas solid [326].

\subsection{Anharmonicities of Individual Phonons}

Experimental measurements of the temperature-dependence of phonon frequencies and lifetime broadenings have been used to assess the relative importances of the cubic and quartic terms of the interatomic potential. These measurements have been performed on insulators such as alkali halides, for which there is no role for thermal excitations of electrons or electron-phonon interactions. A number of studies have used optical spectroscopies, which sample phonons of small $k$. For example, for the transverse optical mode at $k=0$, the cubic and quartic anharmonicities for $\mathrm{AgBr}$ are negative, but positive for TIBr [327]. Neutron scattering studies of phonons at the Brillouin zone boundary in $\mathrm{AgGaSe}_{2}$ with the chalcopyrite structure showed that the anharmonic contributions dominated for an optical phonon, whereas the quasiharmonic approximation was adequate for an acoustic phonon [328].

An anomalously-large phonon damping was found at high temperatures in $\mathrm{CaF}_{2}$, a superionic conductor [329]. The phonon frequencies were approximately consistent with effects of volume expansion, but the Grüneisen parameters for different phonons varied from less than 1 to greater than 3 , and showed some temperature dependence. Especially large non-harmonic effects were found for a mode involving the $\mathrm{F}^{-}$ions. It might be argued that $\mathrm{CaF}_{2}$ is approximately quasiharmonic, but the Grüneisen parameters depend on $T$, and the large dampings of modes are inconsistent with the quasiharmonic model. A recent Raman study of $\mathrm{HfO}_{2}$ showed a similarly large range of mode Grüneisen parameters, and phonon lifetime broadenings that were approximately linear with temperature [317]. By comparing to computational results, the largest anharmonic effects could be attributed to modes dominated by the motions of oxygen ions.

Phonon DOS curves were obtained at several temperatures for both the hP24 and fcc phases of $\mathrm{Co}_{3} \mathrm{~V}$ [121], giving information about how the different phonon frequencies shift with temperature. An average shift can be used to obtain a Grüneisen parameter $\gamma$, and with a thermal expansion measured by diffractometry, a quasiharmonic prediction for the difference in phonon DOS between temperatures $T$ and $T+\Delta T$ is

$$
g_{T+\Delta T}(\varepsilon)-g_{T}(\varepsilon)=3 \gamma \alpha \Delta T\left(g_{T}(\varepsilon)+\frac{\partial g_{T}}{\partial \varepsilon} \varepsilon\right) .
$$

For the two phases of $\mathrm{Co}_{3} \mathrm{~V}$, Eq. 165 gives the two dashed curves in Fig. 35. The solid curves are the actual differences in phonon DOS curves measured at two temperatures. For the hP24 phase, the agreement is perhaps qualitatively correct, but for the fcc phase the agreement is poor. Some ad-hoc adjustment of the Grüneisen parameter for the fcc phase is helpful but not sufficient. For both phases of $\mathrm{Co}_{3} \mathrm{~V}$, the low transverse acoustic modes are more temperature dependent than the others, 


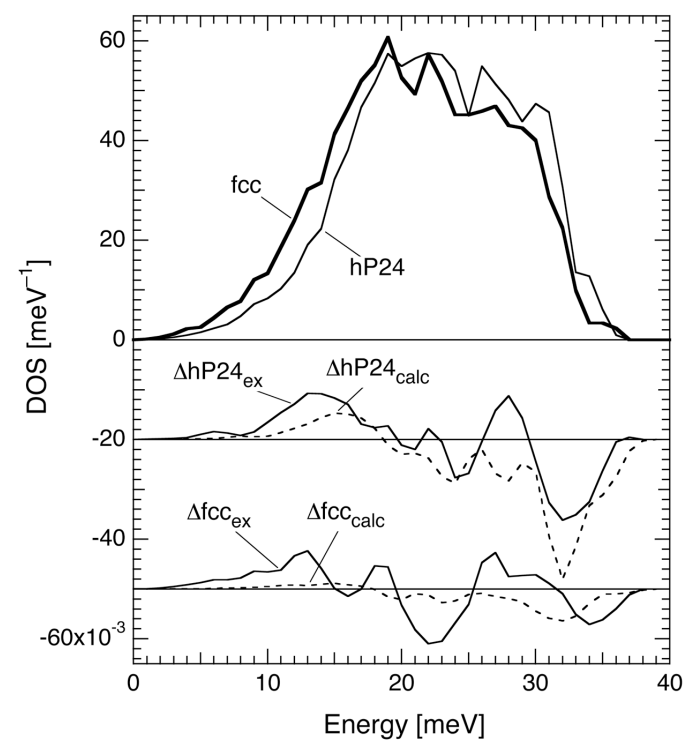

Figure 35. Top: Phonon DOS curves of the fcc and hP24 phases at high temperatures. Bottom: Solid curves labeled $\Delta \mathrm{hP} 24_{\mathrm{ex}}$ and $\Delta \mathrm{fcc}_{\mathrm{ex}}$ are differences between pairs of phonon DOS curves measured at two elevated temperatures (for hP24 at 1308 and $1073 \mathrm{~K}$ ), (for fcc at 1513 and $1333 \mathrm{~K}$ ). Dashed curves labeled $\Delta \mathrm{hP} 24_{\text {calc }}$ and $\Delta \mathrm{fcc}_{\text {calc }}$ were calculated by assuming the same constant Grüneisen parameter for all phonons with Eq. 165. After [121].

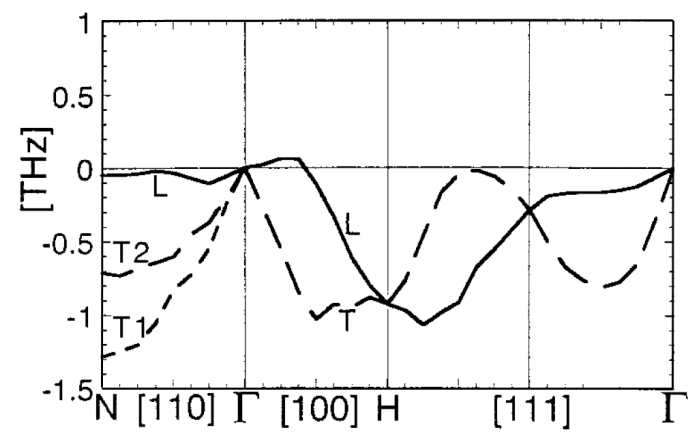

Figure 36. Phonon frequency shifts calculated with a Born-Mayer potential fit to give the correct phonon frequencies of $\mathrm{Zr}$ at high temperature. After [73].

indicating that they are beyond the predictions of quasiharmonic theory, whereas the longitudinal modes seem more quasiharmonic.

Figure 36 shows anharmonic phonon frequency shifts for bcc Zr calculated with perturbation theory for phonons along different crystallographic directions. Approximately, the amount of shift scales with the energy of the phonon, but this varies for the different phonon polarizations and is not an exact relationship. Notice, however, that towards the $\Gamma$ point (i.e., $k=0$ ), the shift goes to zero, as does the phonon energy. There are some discrepancies between the results of Fig. 36 by May, Müller and Strauch [73], and earlier theoretical work by Ye, et al., [103] on bcc Zr.

Ye, Ho, Chen, and Harmon evaluated phonon-phonon anharmonicity in Mo by first-principles frozen phonon calculations. The energies from their density functional theory calculations were used to estimate central force and bond-bending contribu- 
tions to the third and fourth-order anharmonicities of Eq. 146 [104]. The phonon frequency shifts and phonon broadenings were then calculated by the perturbation theory discussed in Section 19.2. An interesting finding was that a central-force model of the anharmonic contributions was inadequate for bcc Mo, although it was successful in their earlier work on bcc Zr [103]. The authors noted that the central force anharmonicity tended to cause phonon stiffening with increased temperature, whereas the bond-bending anharmonicity tended to cause a softening. Even with the addition of bond-bending forces, however, it was found that there was a discrepancy at the (100) H-point when the results were compared to the experimental measurements of Zaretsky, et al., [102]. This direction corresponds to a Fermi surface nesting, and a Kohn anomaly (discussed in Section 20.1.3).

Phonon linewidths are dominated by 3 -phonon interactions in first order, specifically the two middle processes shown in Fig. 34. Some special combinations of phonons interact strongly and decay quickly. For example, in gallium phosphide $(\mathrm{GaP})$ the transverse optical (TO) phonon with $k=0$ can decay into a longitudinal acoustic (LA) plus a transverse acoustic (TA) phonon with opposite momenta, and the energy is matched to that of the original TO phonon. For GaP under pressure, the phonon lifetime broadening was probed by Raman spectrometry [330, 331] The role of pressure was to suppress the matching of energy and momentum for this $\mathrm{TO} \rightarrow \mathrm{LA}+\mathrm{TA}$ process, narrowing the Raman linewidth of the TO mode. The possibilities for matching polarizations, energies, and momenta in 3-phonon processes can be complicated, with special combinations giving "Fermi resonances" such as this one.

Calculating the $k$-dependence of phonon lifetime broadening generally requires rather accurate information about the individual phonon energies. Simple rules for anharmonic phonon lifetime broadening are not yet evident, nor are rules for anharmonic phonon frequency shifts.

\subsection{Anharmonicity and Heat Capacity}

Another approach to finding anharmonicity is by assessing heat capacity data. Although heat capacity can include contributions from electrons and spins, for nonmagnetic insulators such as oxides at high temperature, it is appropriate to assign all the non-harmonicity beyond quasiharmonic behavior to phonon anharmonicity. This was the approach used by Anderson [332], who compiled an appendix on high temperature thermophysical properties of a number of minerals. Two minerals showing substantial anharmonicity are $\mathrm{Mg}_{2} \mathrm{SiO}_{4}$ and Grossular garnet $\left(\mathrm{Ca}_{3} \mathrm{Al}_{2}\left(\mathrm{SiO}_{4}\right)_{3}\right)$ ). Pyrope garnet and an olivine mineral showed modest anharmonicity, whereas $\mathrm{Al}_{2} \mathrm{O}_{3}$, $\mathrm{MgO}, \mathrm{KCl}, \mathrm{CaO}$ did not show much deviation from expected quasiharmonic behavior [332]. Figure 37 shows some of these reduced heat capacity data at high temperatures. The curve for Grossularite shows a particularly striking increase above the harmonic curve (and the classical limit), but it may indicate a change in defect density or another structural change in the material. Studies of phonons in $\mathrm{Fe}_{3} \mathrm{Si}$ alloys at temperatures of 20,930 , and $1100^{\circ} \mathrm{C}$ showed large phonon broadenings, approximately proportional to phonon wavevector [333]. These broadenings were interpreted as effects of chemical disordering in this material, which undergoes an order-disorder transformation at elevated temperatures. 


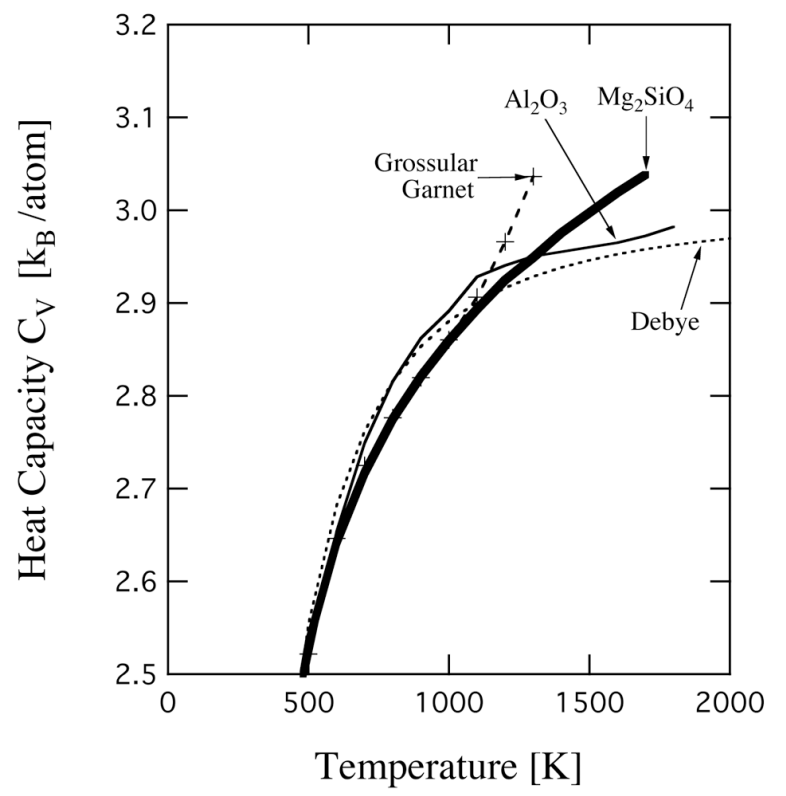

Figure 37. Heat capacity at constant volume $C_{\mathrm{V}}$ for three minerals, and a hypothetical Debye solid with a Debye temperature of 700 K. Data reduced from tables in Ref. [332].

Wallace and colleagues [50] performed a similar thermodynamic analysis for metals, and isolated anharmonic contributions to the entropies. They obtained the quasiharmonic contribution from the effects of thermal expansion on the phonon entropy and on the electronic entropy. Eriksson, Wills, and Wallace performed density functional theory (DFT) calculations of electronic structure that allowed them to account for the electronic entropy by calculating the electronic DOS at $T=0$, and using the Fermi-Dirac distribution to sample the electronic excitations across the Fermi surface [334]. After subtracting the quasiharmonic and electronic entropies from the total calorimetric entropies obtained from the literature, the remainder was the anharmonic entropy attributed to phonon-phonon interactions. This $S_{a}(T)$ is shown in Fig. 38. Differences in $S_{a}(T)$ for fcc and bcc metals are evident in Fig. 38, and supported by further results tabulated in Ref. [50]. The anharmonic entropies of fcc metals tend to be similar, being relatively small and negative. For elemental bcc metals, the anharmonic entropy can be large. There is significant variation in the anharmonic entropies of different bcc metals - Fig. 38 shows substantial differences between Mo and Ta, for example. Heat capacities and other thermodynamic data of Mo and W were assessed by Fernández Guillermet and Grimvall, who reported similarly-large anharmonicities in these bcc metals [335, 336].

Vibrational entropy is fundamentally determined from the volume of phase space in position and momentum coordinates that is explored by the atoms in the material. The phase space explored by coupled anharmonic oscillators is much less well understood than for harmonic solids, and has produced modern research into nonlinear phenomena such as chaos. Nevertheless, it is hoped that the wealth of results on harmonic solids can be adapted to the anharmonic solids. One approach is to use the energies of phonon creations and annihilations measured by inelastic scattering experiments - although the measured phonon spectra show shifts and 


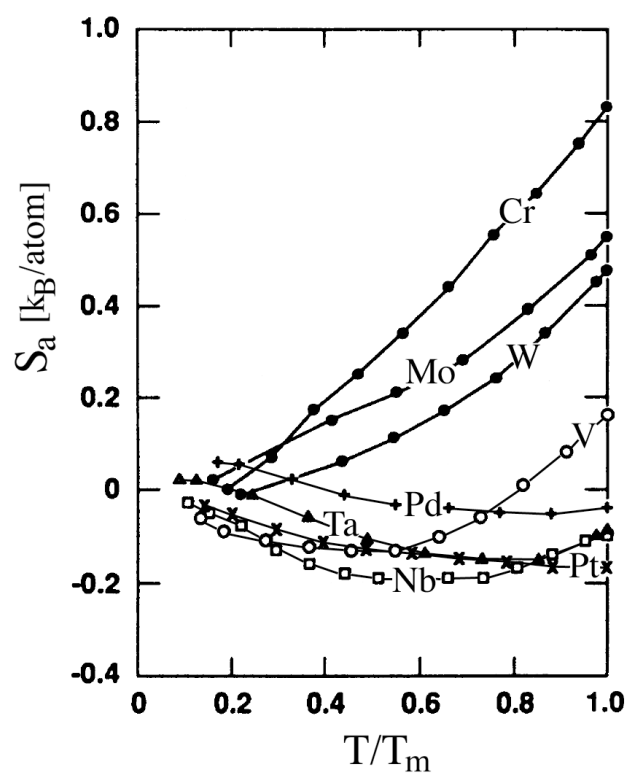

Figure 38. Anharmonic entropy for bcc and fcc transition metals, scaled to the melting temperature $T_{\mathrm{m}}$. After [334].

lifetime broadenings, the experimental phonon DOS measured at high temperature can be analyzed by Eq. 14 to obtain a "quasiharmonic" entropy. Is this approach reliable? Some optimism was offered by Cowley that quasiharmonic theory would be useful for thermodynamic entropy of mildly anharmonic solids [324]. Wallace [46] and others have pointed out that the first order correction for the entropy from anharmonic frequency shifts is

$$
S_{\mathrm{a}}=-\sum_{\vec{k}_{j}} \Delta\left(\vec{k}_{j}\right) \frac{\partial n_{\varepsilon}(T)}{\partial T},
$$

where $\Delta\left(\vec{k}_{j}, T\right)$ is the energy shift of the phonon $\vec{k}_{j}$, and $n_{\varepsilon}(T)$ in the Planck occupancy factor. Using Eq. 14 for the phonon entropy, with the frequencies obtained from inelastic scattering measurements at elevated temperatures, is equivalent in first-order to using a harmonic entropy from the low-temperature phonon DOS plus the contribution from Eq. 166.

This approach does not address any modification to the entropy by effects of phonon damping. Since the damping scales with the cubic term in the potential, by symmetry there is approximate cancellation of contributions in phase space of the position coordinates of opposite signs. Classical damping causes only small shifts of oscillator energies. Damping does cause a change in phonon linewidth, and the broadening of a phonon energy about a mean value is a concern for accurate determinations of vibrational entropy. If $g(\varepsilon)$ is broadened by a damped harmonic oscillator spectral intensity, for example, the vibrational entropy calculated with Eq. 14 will be altered. Nevertheless, if the broadening functions for two materials are the same, e.g., they have the same value of $\mathcal{Q}$ in Eq. 139, their difference in vibrational entropy will be unchanged in the classical limit. Also, for small broadening at least, 
the broadening is approximately symmetrical in $\varepsilon$, and the thermal factors are approximately symmetrical.

Although not fully justified, it is typical to assess the full vibrational entropy at elevated temperature by using Eq. 14 with the $g(\varepsilon)$ measured at the temperature of interest. In the few cases tested to date, this approach seems to work well. Eriksson, Wills and Wallace [334] used neutron scattering phonon measurements at elevated temperatures by Stassis, Petry, Heiming, and co-workers [337, 338, 339, 340] on bcc and hcp Ti, and bcc and hcp Zr to calculate the total phonon entropy at temperature (using Eq. 14). This was subtracted from the entropy obtained from calorimetric measurements, giving the electronic entropy, which was compared to calculation. The ultimate agreement was excellent. For all but bcc Ti, the discrepancy at any temperature was of order $0.01 k_{\mathrm{B}} /$ atom, which is probably better than can be expected from the experimental measurements. For bcc Ti at elevated temperatures, the discrepancy was $0.1 k_{\mathrm{B}}$ /atom or less. Both hcp Ti and hcp $\mathrm{Zr}$ have modestly large anharmonic entropies at their highest temperatures ( 0.35 and $0.43 k_{\mathrm{B}} /$ atom), so the accuracy of the vibrational entropy, obtained from phonon measurements at temperature and analyzed with the harmonic formalism, is most encouraging.

For all materials, phonons make the largest contribution to the heat capacity at high temperatures, and the phonon DOS must be obtained accurately. In cases where the phonon DOS has been measured at elevated temperatures, and the thermodyanamic properties assessed, the anharmonic part of the phonon entropy was generally in good agreement with that deduced from thermodynamic data. The cases of elemental $\mathrm{Ni}$ and $\mathrm{Al}[341,342]$ were in good agreement with the tabulations of Wallace [50], although the contribution from the magnetic entropy of Ni complicates the problem somewhat [343]. The agreement was also good for bcc V [48, 344], but what had been assigned to anharmonic entropy from thermodynamic measurements proved to originate from electron-phonon coupling. It is not easy to separate the effects of anharmonicity from the effects of electron-phonon interactions by experiment alone, but conventional wisdom has been that electron-phonon coupling is negligible at elevated temperatures.

\subsection{Anharmonic Trends of FCC and bCC Metals}

The temperature dependences of phonon frequency shifts and broadenings give insight into the cause of anharmonicity. The quality factor $\mathcal{Q}$ of a damped classical oscillator has no dependence on the amplitude of oscillation, so there is no temperature dependence to its lineshape. This proves inaccurate in general - phonon frequencies usually broaden with temperature. A damping force that depends on the velocity of the nuclei is not fundamental to phonon anharmonicity, of course. From quantum mechanical perturbation theory we expect the first-order lineshape broadening to originate with the cubic term in the potential, and this is calculated to be linear in $T$. Such behavior is sometimes reported, but at higher temperatures the broadening often increases faster than $T^{1}$ (e.g., [333]). Perhaps this can be understood as follows. The potential energy in a nearly-harmonic oscillator increases at $1 / 2 k x^{2} \simeq k_{\mathrm{B}} T$, so the oscillator displacement increases as $x \sim \sqrt{T}$. The limit to this displacement is approximately $x / a=0.15$, where $a$ is the interatomic distance, 


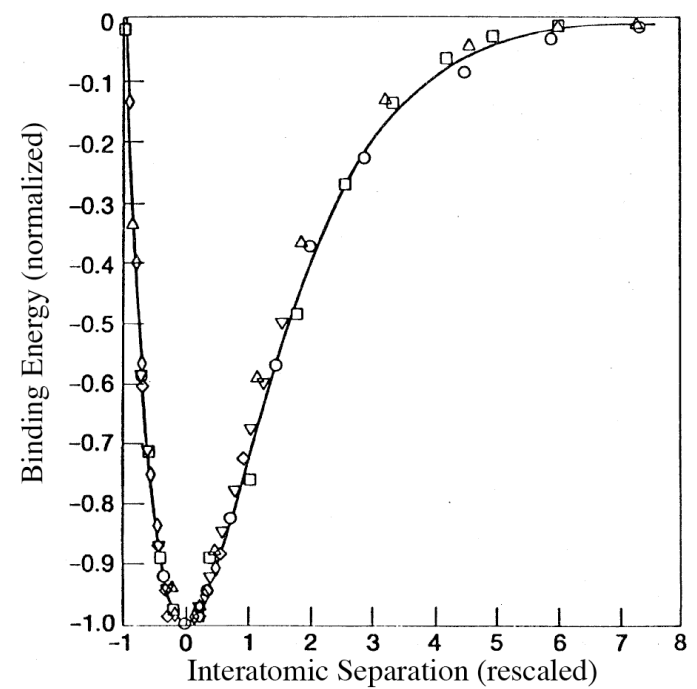

Figure 39. "Universal" interatomic potential of Rose, et al. [345, 346] (solid curve). Points were obtained from Mo, K, Sm, Ba, Cu. After [346].

because larger displacements lead to melting at $T_{\mathrm{m}}$. The maximum value of the cubic term in the potential energy therefore increases as $\left(\frac{T}{T_{\mathrm{m}}}\right)^{3 / 2}$.

It was proposed in the early 1980's that the interatomic potential of different metals, scaled by binding energy, bulk modulus, and with minima centered about the interatomic distance, would follow a universal curve $[345,346]$. The model seemed successful, as shown in Fig. 39. If true, the ratio of the cubic term to the quadratic term in the interatomic potential should be the same for metals. ${ }^{51}$ The constants in Eq. 158 that depend on the interatomic potential are $k$ and $b$. Assuming that the effective $b$ for coupled anharmonic quantum oscillators causes a constant energy spread at a given temperature, then from from Eq. 159 we might expect that $\mathcal{Q} / \sqrt{m} \simeq \frac{\sqrt{k}}{b}$, or

$$
\frac{\mathcal{Q}}{\sqrt{m}} \propto\left(\frac{T}{T_{\mathrm{m}}}\right)^{3 / 2}
$$

for all metals if the phonon linewidth arises from the effects of the cubic term in a universal interatomic potential.

The phonon DOSs of several fcc metals were measured at elevated temperatures by Kresch, et al. [341, 342, 343]. For fcc metals, the high temperature DOS curves could be fit well by modifying the low-temperature phonon DOS curves in two ways. The thermal shifts of phonon energies were small, and modeled successfully as a simple rescaling of the energy axis. The phonon energy broadening was accounted for by a damped harmonic oscillator function with the same value of $\mathcal{Q}$ for all phonons. These simple approximations were surprisingly successful for fcc metals such as Al, as shown in Fig. 40 [343]. The energy rescaling was small and not

${ }_{51}$ When this potential of Rose, et. al., is fit to a polynomial form, the coefficients are comparable for the quadratic and cubic terms in displacement from equilibrium. The quartic term depends on the range of the fit, but is an order-of-magnitude smaller. 


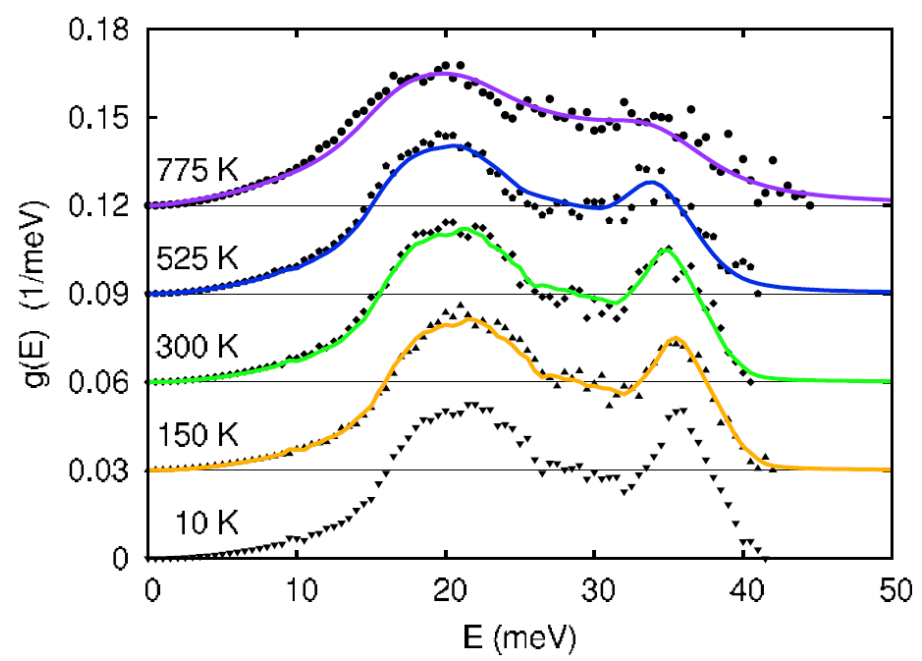

Figure 40. Phonon DOS of aluminum at temperatures as labeled. The markers show the experimental DOS. The lines are best fits to the elevated temperature DOS curves from rescaling the energy axis and convolution with the damped oscillator function Eq. 139. After [342].

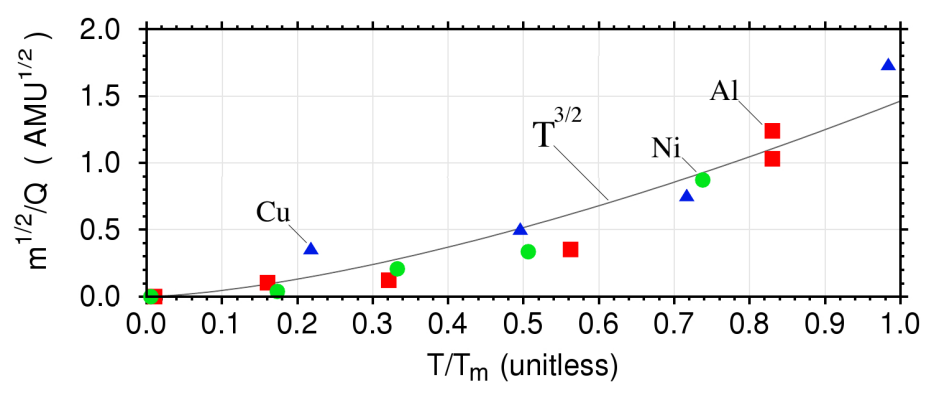

Figure 41. Experimental results from inelastic neutron scattering experiments at elevated temperatures, from which $\mathcal{Q}$ was obtained by fitting Eq. 139 to experimental data from fcc $\mathrm{Al}, \mathrm{Cu}, \mathrm{Ni}$ as shown in Fig. 40. $T_{\mathrm{m}}$ is the melting temperature. After [343].

reliable from experimental measurement, but the trend in the phonon linewidth is presented in Fig. 41. There is a surprising agreement between the experimental phonon lifetime broadening for fcc metals and the predictions of Eq. 167 for a simple model of a "universal" interatomic potential. The reader is cautioned that the data were obtained from only three fcc metals, so there are not enough results on phonon measurements to address the vibrational entropy in a systematic way. Nevertheless, Fig. 41 gives optimism that the phonon anharmonicity in fcc metals may be possible to understand in an general way.

For bcc metals, the trends are less simple because the different phonons do not shift with temperature in the same way [343]. This is particularly true of the low transverse acoustic phonons, which are intrinsically soft for the bcc structure, but vary considerably for different metals. The low transverse acoustic modes tend to soften much more strongly with temperature than the longitudinal modes, and are associated with a number of structural phase transformations such as martensite. The $2 \mathrm{NN}$ distance in the bcc structure is only about $15 \%$ longer than the $1 \mathrm{NN}$ 
distance, so interatomic forces between $2 \mathrm{NN}$ pairs are expected to be important in the bcc structure. This is generally true, based on tabulations of experimental interatomic force constants, for example [347]. These $2 \mathrm{NN}$ force constants can have a strong dependence on temperature, as appears to be the case for bcc $\mathrm{Cr}$ [47], where the longitudinal force constants decrease by a factor of two over the temperature range from 300 to $1773 \mathrm{~K}$, and the low transverse phonon branch shows a prominent softening over this temperature range.

\subsection{ThermoeleCtric Materials}

Thermoelectric materials need to transport electrons efficiently, but conduct heat poorly. Suppressing thermal conductivity is an important design strategy for new materials, and anharmonic effects are believed to be a viable approach to do so. Skutterudite antimonides of typical composition $\mathrm{La}_{1}(\mathrm{Fe}, \mathrm{Co})_{4} \mathrm{Sb}_{12}$ have a cage of $\mathrm{Sb}$ atoms around sites filled by $\mathrm{Fe}, \mathrm{Co}$, and around other sites filled by La. The addition of the caged La atoms suppresses substantially the thermal conductivity, and makes this family of materials interesting for service as a thermoelectric material. The localized vibrational modes of the La atoms were found by inelastic neutron scattering (using neutron-weighting to advantage in the experiment), and their vibrational levels were correlated to the heat capacity of the material [348]. The existence of resonance modes of the La atoms (sometimes called "rattling") is a characteristic of these materials, but with harmonic vibrations it is not obvious how thermal conductivity is suppressed. Subsequent work indicated that although the motions of the La atoms are mainly harmonic, with larger vibrational amplitude they couple more strongly to the acoustic modes responsible for thermal transport [349]. A possibility is that the local modes of the rattling atoms hybridize with the acoustic modes at small wavevector, $k$, effectively reducing the range in $k$ of the linear dispersion of acoustic modes. This would suppress the number of acoustic modes available to transport heat. Another thermoelectric material, $\mathrm{Zn}_{4} \mathrm{Sb}_{3}$ seems to show local modes of dumbell rotation that suppress thermal conductivity [350]. These modes appear to be strongly anharmonic, and may scatter phonons out of the acoustic modes that transport heat.

\section{Electron-Phonon Interactions in Metals}

\subsection{Fermi Surfaces}

\subsubsection{Fermi Surfaces and Electron Screening}

Electron states near the Fermi level affect the interatomic force constants of metals. The electrons and holes screen charge disturbances, minimizing the electrostatic energy. The high effectiveness of this screening helps distinguish metals from insulators. Without screening by conduction electrons, the transfer of an electron between neighboring atoms of differing electronegativity would cause a Coulombic attractive force that falls with distance by about $1 \mathrm{eV} / \AA$ per $\AA$. This contributes about $16 \mathrm{~N} / \mathrm{m}$ to the interatomic force constant, which is comparable to the interatomic force constants themselves. Solutes with substantial differences in electronegativity would 
then cause enormous distortions of interatomic distances and enormous changes in the phonon frequencies. The actual effects of alloying on lattice parameters and phonon DOS curves in metals are much smaller because screening is so effective. Since the atom displacements in a phonon distort the charge density, any changes in screening caused by alloying cause changes in the energy of a phonon. Likewise, it is important to look for changes in screening when considering how the phonons are affected by pressure or temperature. This Section 20 addresses how composition, temperature, and pressure alter the screening.

Electron screening depends on the size and shape of the Fermi surface in $k$-space. For example, the free electron model gives a spherical shape and an electron DOS that tends to be small at the Fermi level. Fermi surfaces are much more complex for transition metals, lanthanides, actinides, and their alloys [351]. Given the same topology of the Fermi surface, however, increasing the electron DOS at the Fermi level will increase screening, softening the phonons. Sometimes the simple parameter of the electron DOS at the Fermi level can be useful for understanding common features of how the phonon DOS is altered by effects of composition. The electron DOS at the Fermi level can also be used to understand the effects of temperature and pressure, although the effects of temperature require additional concepts.

\subsubsection{Fermi Surfaces and the Electron-Phonon Interaction}

To isolate the electron-phonon interaction, we reference the electronic energy to that of an undistorted, periodic structure without phonons and without thermal excitations of electrons. As discussed in Section 18.3, the "adiabatic" electronphonon interaction requires simultaneous thermal excitations of both electrons and phonons. For a free electron gas with a spherical Fermi surface, we expect a strong

contribution from $\hat{H}_{\mathrm{ep}}^{\mathrm{D}}$ of Eq. 154 when $k_{i}=2 k_{\mathrm{F}}$. Momentum conservation requires the three operators in Eq. 154 to give an annihilation of a phonon with wavevector of $2 k_{\mathrm{F}} \hat{\mathbf{z}}$, for example, an electron moved into a state with wavevector $-k_{\mathrm{F}} \hat{\mathbf{z}}$, and an electron removed from a state with wavevector $k_{\mathrm{F}} \hat{\mathbf{z}}$. Both the electron states are at the Fermi level, so at finite temperature there is a good chance that the initial states are occupied and unoccupied as required. For a free electron gas, if $k_{i}$ differs significantly from $2 k_{\mathrm{F}}$, one or more of the electron states is very far from the Fermi surface, and excitation is improbable, even at elevated temperature.

The Fermi-Dirac distribution blurs the Fermi surface. The thermal smearing function that accounts for thermal excitations across the Fermi surface is approximately a Gaussian of standard deviation $2 k_{\mathrm{B}} T$. The available thermal energy also scales with $T$, so the energy of thermal electronic excitations scales as $T^{2}$. The temperature-derivative of this energy is of course the electronic heat capacity, which is linear in $T$. These changes in electronic occupancies can alter the screening characteristics of the conduction electrons in some cases, but the effect can be small in others. For the spherical Fermi surface of a free electron gas, with increased temperature there is only a small change in the numbers of electrons and holes that participate in screening. It is often found that the elastic constants of metals have little explicit temperature dependence at a fixed volume. For many metals, most of the temperature-dependence of the elastic constants originates from the anharmonicity of phonon-phonon interactions, as described in Section 18.2, so effects 
of "adiabatic electron-phonon interactions," as described in Section 20.3.1, are often neglected at high temperatures.

Suppose the electron-phonon interaction is strong. At higher temperatures when more phonons are present, there is a proportionate increase in electron scattering and a shortening of the electron lifetime, $\tau$. A consequence is the lifetime broadening of the electron DOS by the characteristic energy $\Delta E=\hbar / \tau$. This lifetime broadening proves to be tricky to calculate because the increased scattering reduces the strength of the electron-phonon interaction that causes scattering. Nevertheless, in an approximate treatment [352] the resulting electronic broadening function is a Lorentzian function of full-width-at-half-maximum $2 \pi \lambda k_{\mathrm{B}} T$. When $\lambda$ (the electronphonon coupling parameter of Eq. 157) is approximately 1, this lifetime broadening exceeds the energy broadening caused by the Fermi-Dirac distribution. Furthermore, a Lorentzian function has slowly-decaying tails that sample a wide energy spread of the electron DOS.

\subsubsection{Kohn Anomalies and Electronic Topological Transitions}

For more complex Fermi surfaces, the situation is especially interesting when two sheets are parallel, or displaced by a common vector. This structure, sometimes called "nesting" of the sheets of the Fermi surface, allows many electron and hole states near the Fermi surface to be separated by the same wavevector. For a phonon of this wavevector, the screening of the charge disturbance is especially effective, reducing the energy cost of the phonon. This causes a reduction of the phonon energies in a narrow range of wavevector, called a "Kohn anomaly." Many Kohn anomalies have been reported (e.g., [353]).

An "electronic topological transition" can occur when alloying fills (or depletes) the bands with electrons, and a feature of the Fermi surface is created or eliminated [354]. A feature of the Fermi surface for early transition metals is a hole pocket at the center of the Brillouin zone (the " $\Gamma$-point," located at $k=0$ ). Filling it causes a loss of states at the Fermi level and a loss of electrons that can participate in screening. ${ }^{52}$ There are a number of discussions about how electronic topological transitions alter physical properties of metals and alloys such as elastic constants [355, 356, 357, 358, $359,360]$. Electronic topological transitions alter phonons by changing the adiabatic EPI. There have been relatively few systematic experimental studies of these effects, and most previous work was focused on selected phonon branches [361, 362, 363, 364]. An early study of phonon dispersions in single crystals of bcc Nb-Mo alloys showed large changes in phonons at a composition of approximately 66 at.\% Mo that were interpreted with a rigid band model as an electronic topological transition caused by filling the hole pocket at the $\Gamma$-point [361]. At higher $\mathrm{Nb}$ concentrations the picture is more complicated, since an electron "jack" appears in the Fermi surface of Mo around the $\Gamma$-point. Adding Nb to pure Mo causes some phonons to stiffen and some to soften [361]. More recently an electronic topological transition at 30 at.\% Mo was calculated from first principles methods, but in the virtual crystal approximation, and was argued to account for some of the phonon anomalies near

\footnotetext{
52 The electron DOS of vanadium, discussed below in the context of Fig. 43a, shows a drop of a factor of two at $0.35 \mathrm{eV}$ above the Fermi level caused by filling the hole pocket around the $\Gamma$ point.
} 
this composition [365]. A similar effect is expected for W-Re alloys, but effects on phonons have not been studied [366].

Pressure can drive electronic topological transitions, and pressure is particularly convenient for computational work. A computational study on bcc Nb under pressure showed that topological transitions occur at 5 and $60 \mathrm{GPa}$ [367], consistent with measurements of pressure-induced changes in the superconducting transition temperature [368]. A computational study on hcp Zn showed an electronic topological transition at high compression that was responsible for a change in compressibility and the axial ratio of the structure [369]. Experimental evidence for a change in phonon frequencies in $\mathrm{Zn}$ was found by inelastic neutron scattering at a pressure of approximately $6.8 \mathrm{GPa}$ [362], but the effect on elastic constants has been questioned [370]. An enhanced EPI after an electronic topological transition under pressure was recently reported in theoretical work on $\mathrm{YB}_{6}[371]$.

\subsection{Phonons at High-Temperatures in Zirconium, Hafnium, Niobium, Molybdenum, and Chromium}

In a German-French-Austrian collaboration spanning several years, phonon dispersions were measured for single crystals of elemental bcc transition metals at elevated temperatures $[47,339,340,372,373]$. Some of these results were discussed in Section 19.3 as they pertain to phonon-phonon interactions. Other aspects of this work focused on issues of lattice dynamics relevant to self-diffusion and soft-mode transitions. Some important thermodynamic results were also reported in this series of papers. In particular, the phonon DOS curves of $\mathrm{Nb}$ and $\mathrm{Zr}$ showed anomalous behavior with temperature - a slight stiffening of the phonons in both cases, most evident at the Van Hove singularity of the low transverse branch. Electron-phonon interactions were suggested as the source of this behavior. The metal $\mathrm{Cr}$ showed a very rapid softening of the phonon DOS with temperature [47]. An analysis of the lattice dynamics indicated that this originates from the thermal softening of the $2 \mathrm{NN}$ interatomic forces, which are quite large for $\mathrm{Cr}$ at low temperatures. These $2 \mathrm{NN}$ forces have strong effects on the transverse modes of the bcc structure, and seem to be involved in the mechanism of shear transformations. The electronic origin of this softening in $2 \mathrm{NN}$ force constants is less clear, but it may be related to magnetic disorder [374].

A computational study of phonon-phonon interactions in bcc Mo [104] showed some discrepancies from the temperature dependence of phonons measured at the $\mathrm{H}$ point of the Brillouin zone [102]. A slight stiffening with temperature of phonons at the $\mathrm{H}$ point was observed experimentally, but the computational work (without electron-phonon interactions) predicted a large softening. The authors attributed this discrepancy to effects of Fermi surface nesting.

\subsection{Phonons in Vanadium and Its Alloys}

\subsubsection{Temperature Anomalies of the Phonon DOS in Vanadium}

The phonons in pure bcc vanadium have an anomalous temperature dependence Fig. 42 shows that there is no phonon softening to temperatures as high as 1000 $\mathrm{K}$ [48], contrary to predictions of quasiharmonic theory (Section 6.3). On the other 


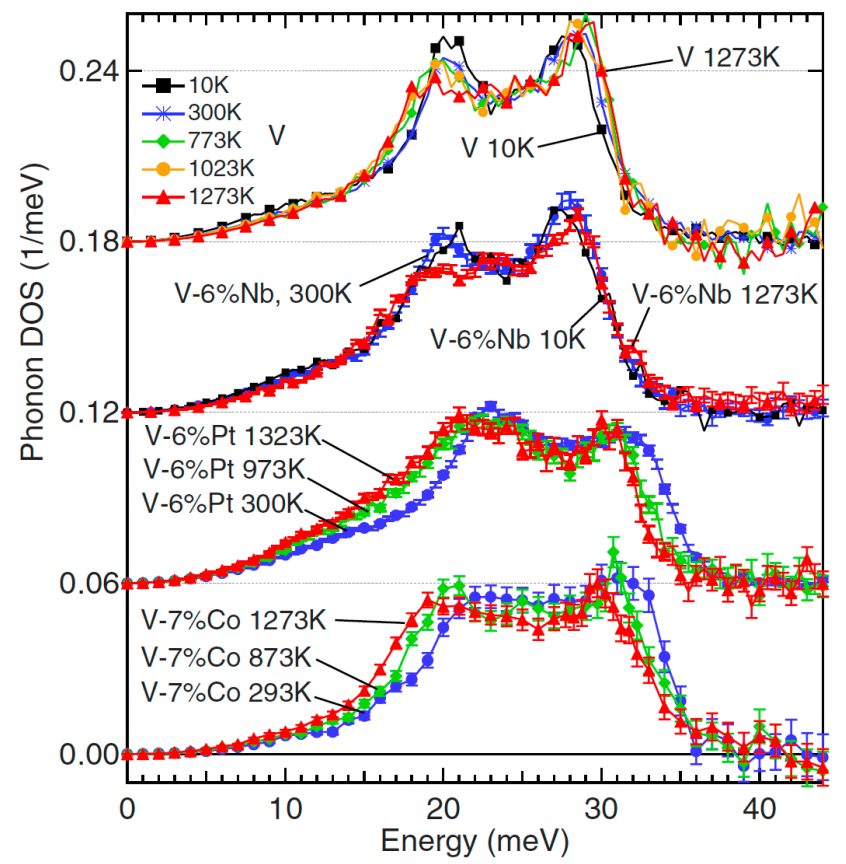

Figure 42. Phonon DOS of $\mathrm{V}$ and $\mathrm{V}$-alloys measured with inelastic neutron spectrometry. After [344].

hand, the phonon DOSs of V-6at.\%Pt and V-Co show a normal softening with temperature that is fully consistent with the quasiharmonic entropy of Eq. 83. It is interesting that $\mathrm{Nb}$ solutes have a minimal effect on the anomalous lack of phonon softening with temperature, but the elements $\mathrm{Nb}$ and $\mathrm{V}$ are isoelectronic, and bcc $\mathrm{Nb}$ and bcc $\mathrm{V}$ have very similar Fermi surfaces.

Figure 43a shows the electron DOS of $\mathrm{V}$ and three alloys, calculated by firstprinciples DFT methods [344, 355]. The addition of $\mathrm{Nb}$ has a negligible effect on the electron DOS, whereas Pt and Co both suppress the electron DOS at the Fermi level. The reduction in the number of states at the Fermi level should suppress the screening of charge density disturbances that occurs in the thermal motions of atoms, thus causing a stiffening of the phonons. This can be seen in the low-temperature data of Fig. 42, but is discussed extensively below.

Figure 43b shows the thermal effects on the electron DOS of V and V-Co [344]. The broadening from the Fermi-Dirac distribution is well known, as is the temperaturedependence of the electron chemical potential, but the broadening from the electron scattering by phonons is less easy to calculate, and its curve at the bottom of the figure must be considered approximate [352]. Nevertheless, both these effects are large, and when convoluted with the electron DOS there is a considerable smoothing of the electron DOS and substantial changes in electron density near the Fermi surface. In essence, the effect of temperature on the electron DOS of vanadium is to reduce the electron DOS at the Fermi level in a way that is similar to the effect of alloying with Co. Both result in fewer electrons that can participate in the screening, 

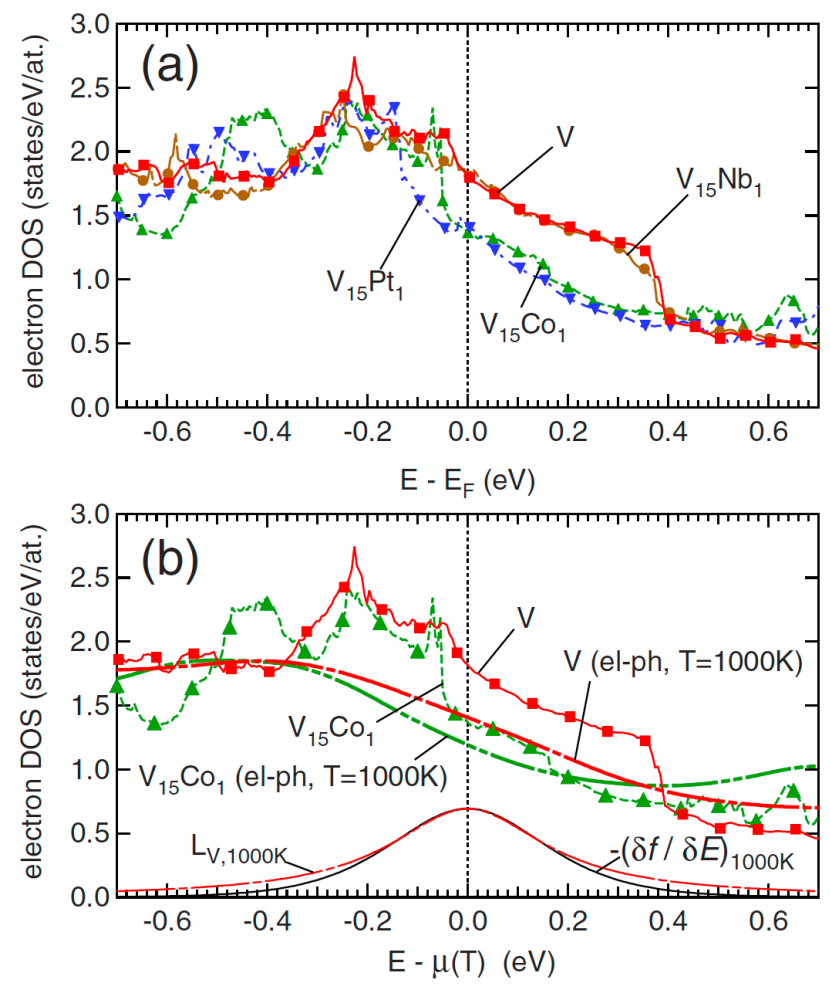

Figure 43. (a) Electronic DOS for $\mathrm{V}$ and $\mathrm{V}_{15} \mathrm{X}_{1}$ computed from first principles with VASP. (b) Electronic DOS for $\mathrm{V}$ and $\mathrm{V}_{15} \mathrm{Co}_{1}$ at $0 \mathrm{~K}$ and with broadening effect induced by the EPI at 1000 $\mathrm{K}$ and shift in $\mu(T)$. Also shown are the derivatives of the Fermi-Dirac function $-\partial f / \partial E$ and the Lorentzian representing the EPI broadening for V, both for $1000 \mathrm{~K}$. After [344].

causing a stiffening of the interatomic force constants. ${ }^{53}$ For the V-Co, Fig. 43b shows less change of the electronic DOS with temperature, and the phonon DOS of V-Co in Fig. 42 shows normal quasiharmonic softening. For pure V, however, the loss of screening with increasing temperature suppresses the softening, effectively stiffening the phonons. The effect is approximately as large as the effects of quasiharmonic softening, although of opposite sign. The two effects cancel approximately, giving a phonon DOS that is largely unchanged over a wide range of temperature.

It was indeed surprising that the effects of electron-phonon coupling in vanadium extend to temperatures as high as $1000 \mathrm{~K}$, since electron-phonon coupling is typically considered in light of phenomena such as superconductivity, and the electron-phonon interaction is typically argued to be negligible at elevated temperatures [50].

\subsubsection{Large Phonon Stiffening in Vanadium Alloys}

Inelastic neutron scattering experiments were used to measure the phonon DOS for bcc solid solutions of $\mathrm{V}$ with $3 d, 4 d$, and $5 d$ transition metal solutes [377, 355]. Results, organized by columns in the periodic table of elements, are presented in Fig. 44. The middle curves, from solutes in the $\mathrm{V}$ column of the periodic table, show

\footnotetext{
53 From prior analysis of the electron-phonon interaction in vanadium, motivated by understanding its superconductivity, it seems that there are only modest differences in screening efficiency of the different electrons at the Fermi surface [375, 376].
} 


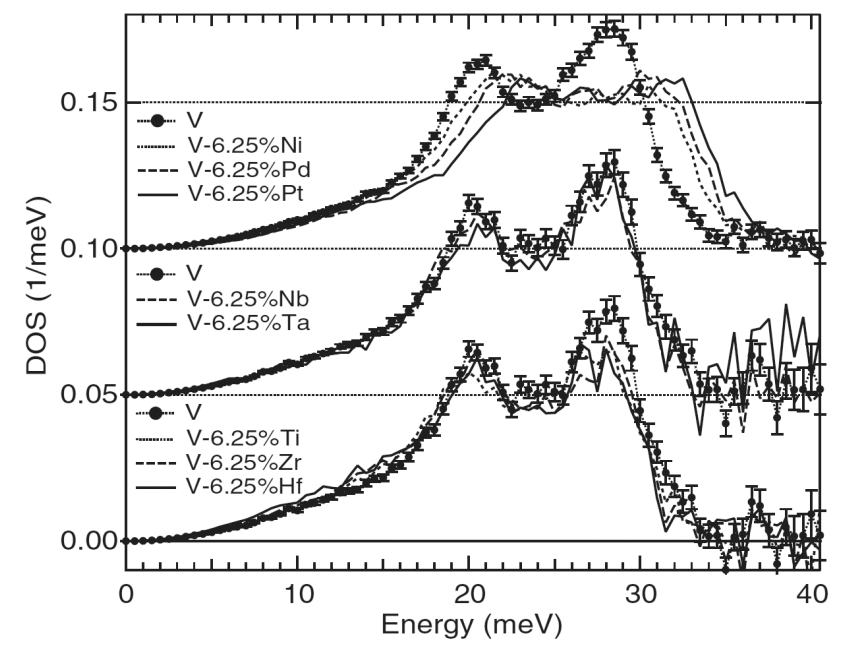

Figure 44. Neutron-weighted phonon DOS for solutes down the Ni, V, and Ti columns. After [377].

little change with alloying. The lower curves, from solutes one position to the left of $\mathrm{V}$ on the periodic table, show a slight softening of phonons with alloying. The top curves, from the elements in the Ni column of the periodic table, show a substantial stiffening. A vibrational entropy of mixing, $\Delta S_{\text {vib }}^{\text {mix }}$, can be defined for a V-X alloy as the difference in vibrational entropy of the alloy and the vibrational entropy of the pure elements $\mathrm{V}$ and $\mathrm{X}$, weighted by their atomic fractions. For dissolving 6.25 at.\% $\mathrm{Pt}$ in $\mathrm{V}, \Delta S_{\mathrm{vib}}^{\mathrm{mix}}$ is $-0.272 k_{\mathrm{B}}$ /atom from Eq. 14 . It exceeds the configurational entropy of mixing $S_{\text {mix }}(0.0625)=+0.234 k_{\mathrm{B}} /$ atom of Eq. 11 . For a V-6.25\% Pt alloy, the total entropy of mixing is therefore negative. Assuming the effects of solute atoms on the phonon spectrum are linear in the solute concentration, as expected at low concentrations, the entropy of mixing will be negative for all concentrations exceeding about $3.4 \% \mathrm{Pt}$. The configurational entropy of mixing will dominate at lower concentrations, owing to the logarithmic singularity of the first term in Eq. 11. Nevertheless, the composition where the total entropy of mixing is zero decreases approximately exponentially with the vibrational entropy contribution of the solute atom. A hypothetical solute causing twice the vibrational effect of $\mathrm{Pt}$ would have a negative entropy of mixing for compositions as low as 0.04 at.\%.

To perform the neutron weight corrections needed for thermodynamic accuracy of these results, a Born - von Kármán model of lattice dynamics was developed for a 16-atom cubic supercell containing 8 BCC unit cells of V atoms, except the central atom was a solute atom. The supercell dynamics allowed calculation of both the true phonon DOS and the experimental neutron-weighted phonon DOS (Sect. 8.3) by iterating interatomic force constants $\Phi_{\alpha \alpha^{\prime} l \kappa l^{\prime} \kappa^{\prime}}$ to find a best fit to the experimental phonon DOS. For the alloy V-6.25at.\%Pt, where the phonons stiffened considerably with respect to vanadium, a large $\mathrm{Pt}-\mathrm{V} 1 \mathrm{NN}$ longitudinal force constant was found responsible for the change in phonon DOS of Fig. 44. The changes in the total DOS 


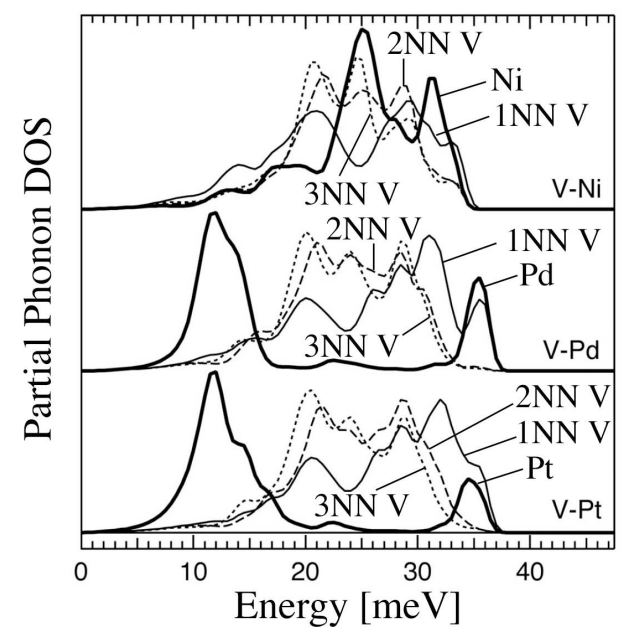

Figure 45. Partial DOS curves for the solute atom and its V neighbors up to 3NN shell, obtained from the optimized supercell lattice dynamics. After [378].

were dominated by the partial DOS of $\mathrm{V}$ atoms having solutes in their $1 \mathrm{NN}$ shell (Fig. 45). ${ }^{54}$

Figure 46 shows a good correlation between the vibrational entropy of alloying and the electronegativity of transition metal solutes across the $3 d$ row, and with $4 d$ and $5 d$ solutes down several columns of the periodic table. First-principles calculations on supercells matching the experimental compositions predicted a systematic charge redistribution in the nearest neighbor shell around the solute atoms. The charge transfers between atoms were evaluated with the Bader algorithm (which tends to overestimate the charge transfer in metals, although it follows the Pauling electronegativity scale). Although this correlation points to the importance of electronic rearrangements on phonon frequencies (as opposed to atomic size or mass, for example), more fundamentally it is the screening of ion motions by the electron density that governs the restoring forces on the ions and hence the phonon frequencies (Sect. 20.1.1 and [379]). The $d$ states of the late transition metal solutes $\mathrm{Ni}$, $\mathrm{Pd}$, and $\mathrm{Pt}$ are lower in energy than the rest of the alloy valence bands, leading to a split band for $\mathrm{Pd}$ and $\mathrm{Pt}$ solutes and considerable distortions of the band shape. Correspondingly, there is a systematic depletion of the number of states at the Fermi level for solutes toward the end of the transition metal series (about 20\% for 6-7\% solutes from the Ni column). This will reduce the number of weakly localized states available for screening the displacements of vanadium ions, causing higher energies for these displacements and higher phonon frequencies. The efficacy of screening depends on the available electronic excitations. The change with temperature of the screening can be attributed to an adiabatic electron-phonon interaction (EPI) as discussed next, even though the temperatures are quite high. ${ }^{55}$

\footnotetext{
54 Note also the resonance modes (Sect. 3.1) in the partial DOS curves of Pd and Pt around $12 \mathrm{meV}$.

${ }^{55}$ It has been argued that the EPI is negligible above about a quarter of the Debye temperature of vanadium [17].
} 


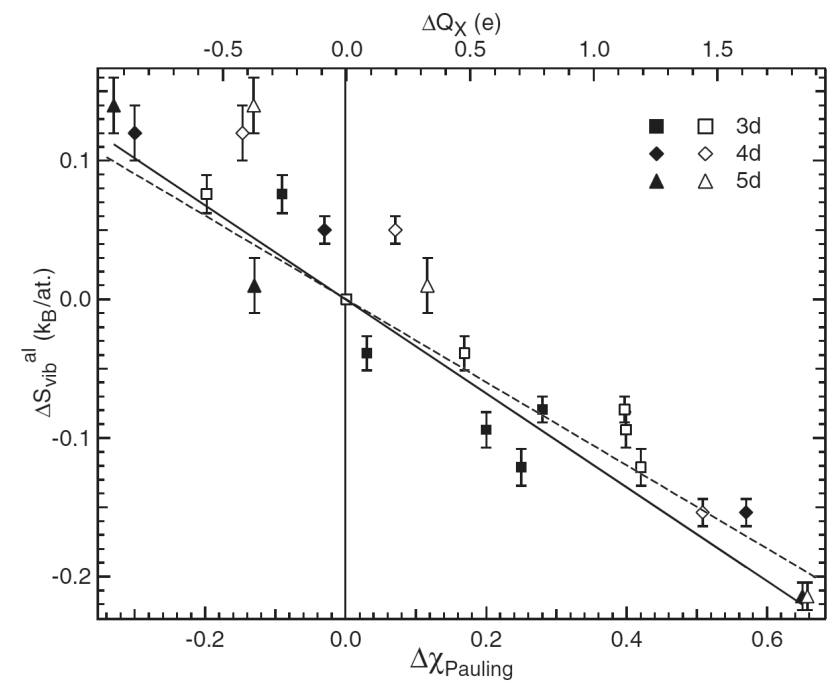

Figure 46. Neutron-weighted vibrational entropy of alloying, $\Delta S_{\mathrm{vib}}^{\mathrm{al}}$, plotted versus difference in Pauling electronegativity $\Delta \chi$ (solid symbols) between solute and $\mathrm{V}$, and Bader charge transfer on solute $\Delta Q_{\mathrm{X}}$ (open symbols). The solid (dashed) line is a linear fit of $\Delta S_{\mathrm{vib}}^{\mathrm{al}}$ versus $\Delta Q_{\mathrm{X}}$. After [377].

\subsection{The Adiabatic EPI in Superconductors}

Figure 47 shows changes with temperature of phonon DOS curves of three compounds with the A15 structure [380]. The compounds $\mathrm{V}_{3} \mathrm{Si}$ and $\mathrm{V}_{3} \mathrm{Ge}$ are well-known superconductors (with strong electron-phonon coupling), but $\mathrm{V}_{3} \mathrm{Co}$ is not. The compound $\mathrm{V}_{3}$ Co shows a normal phonon softening with temperature that is approximately consistent with predictions of quasiharmonic theory. On the other hand, the phonons in $\mathrm{V}_{3} \mathrm{Si}$ actually stiffen with increasing temperature! This stiffening is weaker for $\mathrm{V}_{3} \mathrm{Ge}$, which has a lower critical temperature for superconductivity. Figure 48 shows the temperature dependence of the average phonon frequency for $\mathrm{V}_{3} \mathrm{Si}$ and $\mathrm{V}_{3} \mathrm{Ge}$. The average phonon energy first increases with temperature, then decreases at high temperatures. The low-temperature behavior is attributable to anomalously soft phonons owing to a strong electron-phonon interaction (EPI). With increasing temperature the EPI is suppressed, and quasiharmonic behavior is expected at the highest temperatures.

The origin of the thermal suppression of the electron-phonon interaction is indicated in Fig. 49b, where the electron DOS at the Fermi level is shown to be strongly reduced with temperature for $\mathrm{V}_{3} \mathrm{Si}$. This is caused by a thermal broadening of the electron DOS from the Fermi-Dirac distribution function, the thermal shift of the electron chemical potential, and from the shortened electronic lifetime at elevated temperatures. Figure $49 \mathrm{a}$ shows that in contrast, $\mathrm{V}_{3}$ Co does not have much structure of its electron DOS at the Fermi level, and it is found that the changes with temperature are small.

Figure 50 shows phonon DOS curves for $\mathrm{V}_{3} \mathrm{Si}$ calculated with ab-initio methods [380]. The middle curves of Fig. 50, calculated with the thermal broadening of electronic levels, are generally consistent with the experimental observation of phonon stiffening, especially for the low-energy modes in which the motions of $\mathrm{V}$ atoms are 


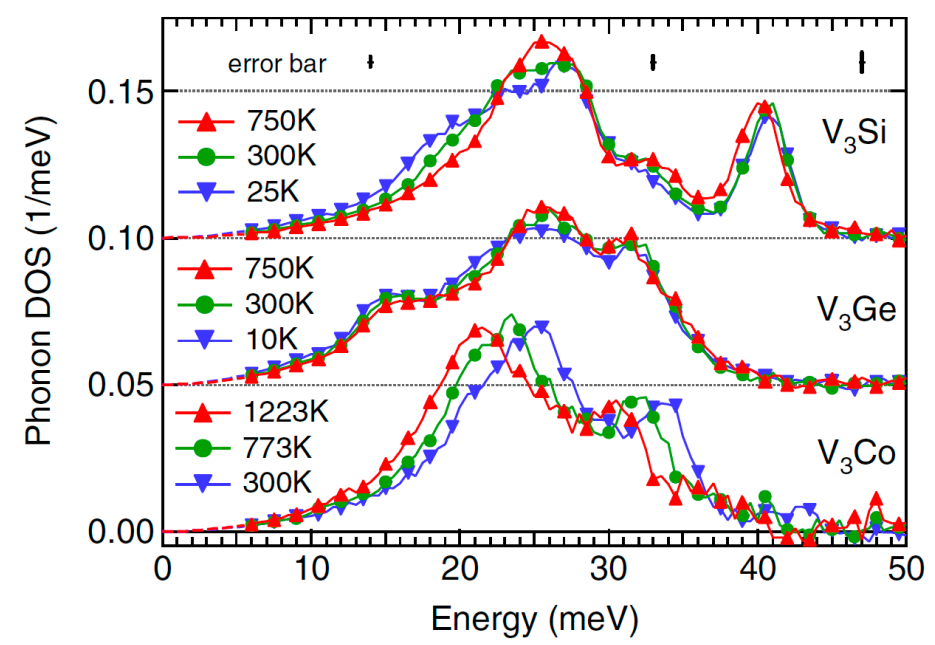

Figure 4\%. Phonon DOS of A15 measured with inelastic neutron scattering, at different temperatures. After [380].

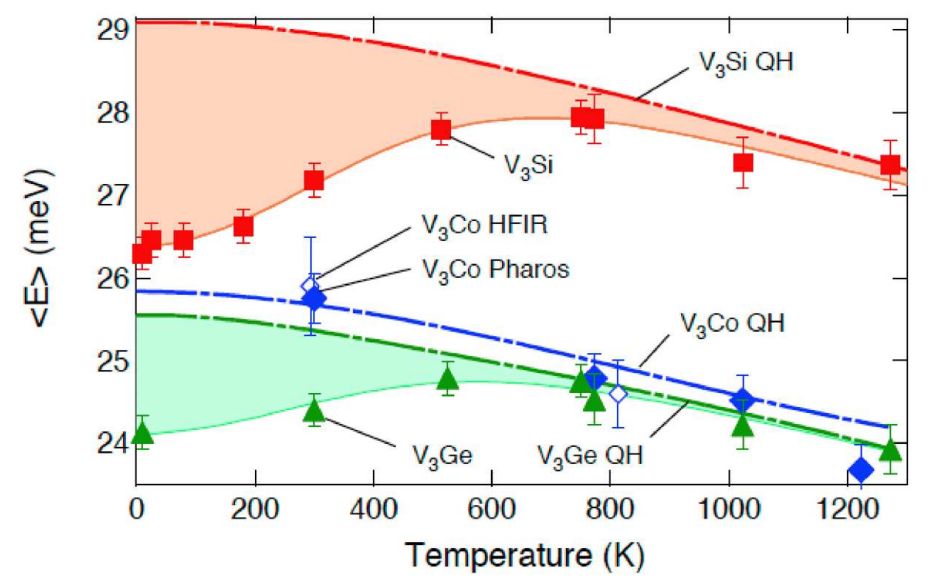

Figure 48. Average phonon energy of $\mathrm{A} 15$ compounds of $\mathrm{V}_{3} \mathrm{Si}, \mathrm{V}_{3} \mathrm{Ge}$, and $\mathrm{V}_{3} \mathrm{Co}$ as functions of temperature (from inelastic neutron scattering measurements). Markers are results from INS measurements. Dash-dotted lines correspond to conventional quasiharmonic $(\mathrm{QH})$ behavior. The $\mathrm{QH}$ curves were offset vertically to match the experimental data at the highest $\mathrm{T}$ measured. After [380].

dominant. In contrast, the top curves show that volume expansion alone causes a softening of the phonons corresponding approximately to a rescaling of the energy axis. This is as expected with the quasiharmonic model, but is inconsistent with the experimental trend. At the bottom of Fig. 50 are phonon DOS curves calculated with consideration of the effects of both thermal expansion and the adiabatic EPI. These agree very well with the experimental trends. It is interesting that the lifetime broadening function required to fit the experimental trend is a bit larger than expected, which suggests that the electron-phonon coupling parameter $\lambda$ is suppressed with temperature. 


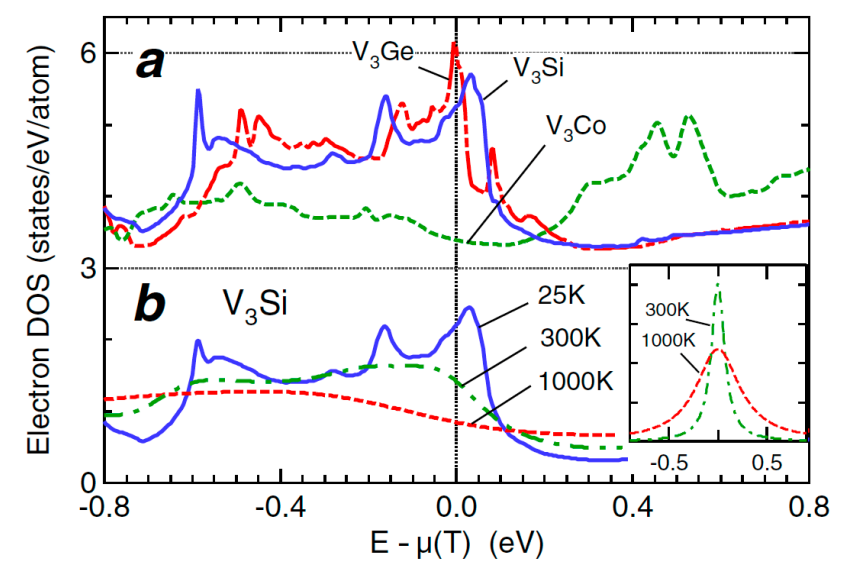

Figure 49. (a) Electronic DOS for $\mathrm{A} 15 \mathrm{~V}_{3} \mathrm{Si}, \mathrm{V}_{3} \mathrm{Ge}$, and $\mathrm{V}_{3}$ Co from first-principles VASP calculations. (b) Electronic DOS for $\mathrm{V}_{3} \mathrm{Si}$ broadened by EPI at $25 \mathrm{~K}, 300 \mathrm{~K}$, and $1000 \mathrm{~K}$, using electron-phonon coupling parameter $\lambda=1.0$ at all T. Energies are with respect to the electron chemical potential $\mu(T)$. Inset: broadening function at $300 \mathrm{~K}$ and $1000 \mathrm{~K}$.

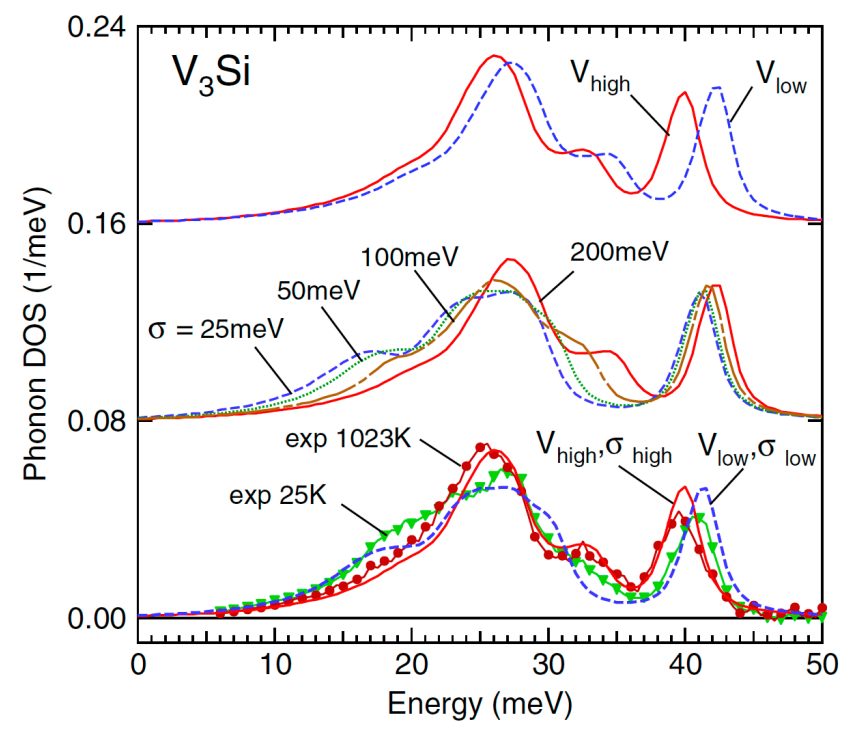

Figure 50. Top: Phonon DOS for $\mathrm{V}_{3} \mathrm{Si}$ calculated from first principles for two different volumes $\left(V_{\text {high }}=1.03 \times V_{\text {low }}\right)$. Middle: Calculated DOS for different electronic broadenings $\sigma$. Bottom: The same as above for two different $(V$ and $\sigma)$ combinations $\left(\sigma_{\text {low }}=50 \mathrm{meV}\right.$ and $\left.\sigma_{\text {high }}=200 \mathrm{meV}\right)$. The calculated DOS was obtained from the neutron-weighted contributions of $\mathrm{V}$ and Si species and convolved with the experimental instrument resolution. The markers are experimental results for $\mathrm{V}_{3} \mathrm{Si}$ at 25 and $1023 \mathrm{~K}$.

Perhaps the most surprising result is that the EPI is thermodynamically important to $1000 \mathrm{~K}$ in $\mathrm{A} 15 \mathrm{~V}_{3} \mathrm{Si}$ and $\mathrm{V}_{3} \mathrm{Ge}$, defying much conventional wisdom and many calculations of the effect of the EPI on the free energy of metals [50,352]. Such a possibility was suggested by a few early theoretical papers that addressed EPI effects on the heat capacity [381]. More recent calculations on free-electron metals without structure in the electron DOS near their Fermi levels found effects of the adiabatic EPI at high temperatures, but these effects were small [382, 383, 384] (discussed 
in Section 20.5). Other studies of early transition metals [385] and superconductors [386] suggested the importance of electron-phonon interactions on phonon dynamics.

Phonons in the C32 phase of $\mathrm{MgB}_{2}$ have been studied thoroughly at low temperatures, since the (nonadiabatic) EPI is the origin of its conventional superconductivity. It is possible to fully substitute $\mathrm{Al}$ for $\mathrm{Mg}$ [387], obtaining the same C32 structure across the $(\mathrm{Mg}, \mathrm{Al})^{11} \mathrm{~B}_{2}$ "quasibinary" system, and $\mathrm{C} 32 \mathrm{AlB}_{2}$ is not superconducting. Phonons have been studied with different $\mathrm{Al}$ and $\mathrm{Mg}$ concentrations [388], and the superconducting Mg-rich alloys show considerable softening of the high-frequency optical modes, consistent with a strong EPI and larger vibrational entropy. Early work indicated that superconductivity in $\mathrm{MgB}_{2}$ [389] originated with a strong EPI caused by anharmonic $E_{2 g}$ phonons. Later this interpretation has been called into question, and it has been argued that $\mathrm{MgB}_{2}$ is not particularly anharmonic [390]. Furthermore, the adiabatic approximation itself has been questioned because the electrons may not respond instantly to nuclear displacements [390]. A limitation of the previous work on phonon anharmonicity in $\mathrm{MgB}_{2}$ is that the measurements were at temperatures of $300 \mathrm{~K}$ and below, such as the extensive Raman spectrometry work that has been performed on $\mathrm{MgB}_{2}$ and related materials [388, 391, 392, 393, 394].

\subsection{Effects of Pressure, Composition, and Temperature on the Adiabatic EPI And the Phonon DOS}

For understanding how changes in the adiabatic EPI cause changes in phonons, we need some level of information about changes in the electronic structure near the Fermi level. If similar changes are caused by either temperature, composition, or pressure, we expect similar changes in electron screening, and similar effects on the phonons. For a simple example, increasing the band filling by alloying should have the same effect on the elastic moduli as a band filling caused by a reduction in volume with pressure ${ }^{56}$ For simple free-electron metals the effect of temperature is different, however, being a smearing the electron DOS.

Bock, Coffey, and Wallace considered the adiabatic and non-adiabatic electronphonon interactions with results from a pseudopotential perturbation theory [382, $383,384]$. In essence, free electron theory was extended with perturbations from pseudopotentials, and perturbations from nuclear displacements, giving effects from the EPI that were second order in the pseudopotential. The non-adiabatic EPI was found to be small, in the range of $10^{-5}$ to $10^{-4} \mathrm{eV}$ (but increasingly larger for $\mathrm{K}$, $\mathrm{Na}, \mathrm{Pb}$, and $\mathrm{Al}$ ), and weakly decreasing with temperature. The adiabatic EPI was calculated with phonon dispersions from experimental lattice dynamics, and with simple free electron dispersions for the electronic structure. For a reference state of a perfectly periodic crystal with no electronic excitations, the dominant effect of temperature was from the Fermi-Dirac distribution, $f(T)$, which gave a factor of $T^{2}$, and from the phonon occupancy factor $n(\varepsilon, T)$, which gave a factor of $T^{1}$ at modest temperatures. The numerical results for the adiabatic EPI were negligible at low temperatures, but increased as $T^{3}$ and exceeded the non-adiabatic EPI at the melting temperatures. Except for $\mathrm{Al}$, however, the non-adiabatic EPI was a

\footnotetext{
${ }^{56}$ For a free electron gas with a spherical and featureless Fermi surface, we need only the electron DOS at the Fermi level to predict, for example, that the bulk modulus scales with the electron DOS at the Fermi level.
} 
small effect. For $\mathrm{Al}$ at melting, the adiabatic EPI was $10^{-3} \mathrm{eV}$, which corresponds to an entropic effect of order $10^{-2} k_{\mathrm{B}}$ /atom. It seems that the adiabatic EPI for free electron metals is not so important thermodynamically, but it is within an order of magnitude of being so.

Metals with sharp features at the Fermi surface have larger thermal effects from the adiabatic EPI. The thermal broadening of the electron DOS can be understood as taking sharp peaks near the Fermi level and leveling them, or alternatively as filling states at low points in the DOS. We might expect that the detailed 3D structure of the Fermi surface could be important for understanding how phonon frequencies are affected by the adiabatic EPI, but the thermal effects of Sects. 20.3.1, 20.3.2, and 20.4 showed good correlations to the simpler density of states at the Fermi level. For example, for $\mathrm{A}_{15} \mathrm{~V}_{3} \mathrm{Si}$ Fig. 49b shows that the Fermi level is at a peak in the electron DOS, so the thermal smearing and the shift in electron chemical potential reduce markedly the numbers of electrons and holes available for screening, reducing the EPI. Semiquantitatively, at least, this contributes more to the stiffening of phonons than in the case of pure bcc V, where Fig. 43 shows a more mild effect of temperature. The electronic DOS curves of the A15 compounds and bcc V are similar, however, with a Fermi level crossing the upper edge of a peak in the DOS. ${ }^{57}$

Nevertheless, generalizations of the effects of pressure, composition and temperature may be risky. In particular, under pressure the electron states remain well defined and long-lived. With increased pressure a complex Fermi surface is expected to remain anisotropic, and retain its complex features. With temperature, however, the electrons scatter out of their ground states owing to interactions with phonons, giving a lifetime broadening of the electron energy levels. A similar effect is expected from the effects of composition (if solutes are treated as scattering electrons from the states of a pure element). Intricate structures of the Fermi surface at $T=0$ will be lost at high temperatures owing to thermal broadening of the electron energies, however. The lifetime broadening causes a more interesting effect on the states at the Fermi level that participate in the EPI:

- The lifetime broadening of the states at the Fermi surface owing to adiabatic electron-phonon interactions is dependent on the strength of the electronphonon interaction in different directions in $k$-space.

- Those directions in $k$-space with the stronger electron-phonon interactions will broaden more with temperature, suppressing the electron-phonon interaction.

- The suppression of the electron-phonon interaction along directions in $k$-space where it is strong will tend to equalize the strength of the electron-phonon interaction in all directions.

The thermal smearing of the electron states and the increased isotropy of the electron-phonon interaction will therefore wash out the detailed structure of the Fermi surface. For a Fermi surface with one sheet, its low-temperature structure

57 It is quite possible for the opposite effect to occur. When the Fermi level lies in a valley between regions of high electron DOS, it is expected that temperature will increase the number of electrons available for screening, and cause a further softening beyond what is expected from quasiharmonic behavior. 
will evolve towards a fuzzy sphere at high temperatures. For a Fermi surface with isolated pockets, the anisotropy may persist to higher temperatures, but the detailed systematics require further research.

Owing to the complexity and different structures of Fermi surfaces, further studies are needed to understand how well the effects of temperature on vibrational entropy can be predicted with only the electron DOS near the Fermi level. Furthermore, some conceptual issues such as how best to account for the lifetime broadening of electron states remain a challenge. Nevertheless, our present understanding of the concepts suggests that studies of phonons at high temperatures may provide useful information about the EPI, and perhaps about superconductivity, although the latter is more sensitive to the anisotropies of the EPI. 
Vibrational Thermodynamics of Materials

\section{Overview and Summary}

\section{Systematic Trends of Vibrational Entropy}

\subsection{Multiple Effects}

For solid-solid phase transformations, vibrational entropy has several contributions that are responsible for systematic behaviors.

- It is important to count the different types of atom pairs in an alloy, and how they change in the phase transformation. The interatomic forces for $1 \mathrm{NN}$ pairs must be known. In many cases the effects of the $1 \mathrm{NN}$ triangle and $2 \mathrm{NN}$ pairs are important, but there is cause for optimism that larger clusters make rapidly-diminishing contributions.

- Bond stiffnesses depend on bond lengths, and these effects can be comparable to differences in stiffness between different atom pairs. Effects of bond angle are not fully explored, but may be important for materials with $d$ - and $f$-electrons.

- Comparisons of bond stiffnesses are more difficult when there is a change in the crystal structure. The $2 \mathrm{NN}$ force constants are much larger in the bcc than the fcc structure, for example.

- With changes in composition, not all effects can be attributed to stiffness changes of local bonds, because changes in the electron density at the Fermi level cause non-local effects that depend on the overall composition.

- Alloying and pressure can be understood as changing the electronic structure near the Fermi level, which causes changes in the screening of charge disturbances that accompany the vibrations of atoms. Modern DFT calculations can predict these changes quantitatively in many cases.

- "Quasiharmonic behavior," is a classic thermal response that can be predicted from the bulk modulus and coefficient of thermal expansion. It reflects how the bond stiffness depends on bond length, and originates with the non-quadratic parts of the interatomic potential. Nevertheless, the interatomic potential is approximated as a harmonic one that changes with temperature.

- Anharmonicities from phonon-phonon interactions originate from an interatomic potential that is unchanged with temperature. The phonon lifetime broadening is dominated by the cubic part of the potential, and may have a common behavior for fcc metals. The phonon frequency shift depends on both the cubic and quartic terms of the potential, and trends are less obvious to date.

- The adiabatic EPI accounts for how thermal excitations of electrons in metals interact with thermal excitation of phonons. For free electrons, the strength of the adiabatic EPI scale as $T^{3}$, but larger thermal effects with different

temperature dependences occur when there is big structure of the electron DOS 
near the Fermi level. Pressure and composition can cause similar effects on the electron screening.

\subsection{Hume-Rothery Rules and Vibrational Entropy}

The long previous list of phenomena affecting vibrational entropy may discourage simple rules of thumb, but simple rules are used for other quantities in alloy thermodynamics. Assessing differences in vibrational entropy by rules analogous to Hume-Rothery rules $[153,395,396]$ for metallic alloys risks predictions that are no better than the Hume-Rotherey rules for enthalpy, and likely worse. The Hume-Rothery rules of alloy phase formation are based on atomic properties: 1) electronegativity, 2) metallic radius, and 3) electron-to-atom ratio, with the first two factors being the most important. For phonon entropy, we should add 4) atom mass, to this list.

- Electronegativity. This is a measure of the tendency for electron transfer to an atom during chemical bonding, which provides information on the strength of the bond and its stiffness. In metals this is only a part of the picture, because conduction electrons are highly effective in screening the charge disturbances that occur with electron transfer, but changes in the screening are also expected to scale with differences in electronegativity. Screening depends on the Fermi surface, but we expect systematic trends with electronegativity if, for example, the band structure is little changed with alloying.

- Metallic Radius. Inserting a larger solute atom will cause a local compression in the host crystal. Since interatomic forces generally become stiffer under compression, alloying with large atoms tends to reduce the vibrational entropy.

- Electron-to-Atom Ratio. This accounts for non-local Fermi surface effects in metals. The e/a ratio is expected to be a useful parameter if the rigid band model is reasonably reliable, and could be used to predict changes in screening, for example.

- Mass. There is a general relationship for an oscillator of $\omega=\sqrt{k / M}$, where the frequency, $\omega$, scales as the inverse root of the mass, $M$, assuming that the spring constant, $k$, is in fact a constant. Across the periodic table the atomic mass varies from 1 to 238 and beyond, and for a matrix having atoms of mass $M_{0}$, and solutes having mass $M_{S}$, with respect to the host the phonon entropy of the alloy scales with $\ln \left(M_{0} / M_{S}\right)$ [206]. No effects of mass are expected when a phase transformation rearranges the same atoms into a different structure, however. 


\section{Future Directions}

\subsection{OPPORTUNITIES FOR EXPERIMENT}

Measurements of the phonon DOS have long been possible with Fermi chopper spectrometers, but the experimental situation has changed markedly with the advent of new and efficient facilities for inelastic neutron scattering. There is now enough intensity so that dispersive information from coherent scattering can be measured reliably over wide ranges of $Q$ and $E$. For powder samples this $Q$-information is rich in detail, but it is averaged over different crystallographic directions. With computational support, the $S(Q, E)$ from polycrystalline samples can progress towards the more detailed information obtained from single crystals. Nevertheless, single crystal studies are possible with chopper spectrometers. With measurements at multiple crystal orientations, these studies should prove an efficient way to obtain phonon dispersions. Improved rates of data acquisition now allow more systematic measurements of phonons at elevated temperatures and modest pressures of a few GPa.

A triple axis spectrometer is highly accurate in its energy and momentum measurements, and when a full set of phonon dispersions are measured on single crystals of ordered compounds, the phonon DOS derived from a lattice dynamics model is highly reliable. This technique is not so reliable for disordered solid solutions, unfortunately, because the dispersions are inherently broadened, and this broadening need not be symmetrical in energy. Nevertheless, the biggest challenge remains obtaining single crystals of the material of interest. On the horizon are high intensity beams of long wavelength neutrons, which should offer new opportunities for studying long wavelength and low energy excitations in mesoscopic systems. These cold neutrons may also provide better energy resolution for phonon studies using phonon annihilations.

Phonon dispersions can now be measured on very small single crystals by inelastic x-ray scattering. In spite of flux limitations, these experiments are practical today for many chemical compositions. This method could make significant advances with modest investments in energy analyzer systems. Inelastic nuclear resonant scattering is an incoherent scattering technique for determining phonon partial DOS curves of isotopes such as ${ }^{57} \mathrm{Fe}$ and ${ }^{119} \mathrm{Sn}$, and is reasonably mature. Both these synchrotron methods offer the opportunities for measuring phonon information under extreme conditions of pressure and temperature, and these opportunities are starting to be exploited.

\subsection{Opportunities For Theory}

At low temperatures it is possible to use electronic structure calculations with the local density approximation to calculate phonon frequencies with good accuracy, but a $1 \%$ accuracy remains a challenge for many systems. Nevertheless, presentday DFT calculations are invaluable for finding trends in vibrational entropy with composition and pressure, and will see more widespread use. Better methods for electronic structure calculations are under active development. Improvements in ab-initio calculations on magnetic systems are expected as the exchange energy is 
handled by methods beyond the local density approximation, but the cooperative nature of the magnetic transition or the magnetic susceptibility remains a computational challenge. Density functional theory is not on solid ground when electronic excitations are considered, but there are developments in time-dependent DFT methods, and GW calculations give a more rigorous correlation energy. Path integral quantum Monte Carlo methods are also under development. These methods should prove valuable when considering matter in extreme conditions [397, 398]. Many of these computational methods will benefit from the ongoing rapid improvements in computing capabilities. Quantum molecular dynamics offers opportunities to handle anharmonic forces in a fundamental way, and with better computing resources these ab-initio molecular dynamics studies will be practical for larger systems and longer times.

At modest temperatures, the suppression of the electron DOS near the Fermi level owing to the effects of electron lifetime is not well understood. The adiabatic electron-phonon coupling can be obtained from band structure calculations on supercells with atoms in static positions characteristic of thermal displacements. Results from an ensemble of such calculations give a broadening of the electron band structure that increases with temperature. Better calculations on the adiabatic electron-phonon interaction can be used for more detailed predictions of thermal effects along different crystallographic directions. It may be possible to do this self-consistently, so the directions with the stronger EPI are more sensitive to temperature.

The dynamics of disordered, nonlinear, or anharmonic systems have a long history. The entropy of anharmonic systems will likely remain a challenge for many years to come. In the interim, it seems possible that better molecular dynamics calculations, perhaps ab-initio calculations, will play an increasingly important role in sorting out the dynamics of anharmonic materials and their temperature dependences.

\subsection{OUtLOOK}

Much of the subject of vibrational thermodynamics of materials is based on the statistical mechanics of harmonic oscillators. It is fortunate that the harmonic approximation gives a good start in understanding basic trends, because there are so many useful and powerful mathematical results for harmonic vibrations. Deviations from harmonicity are important, however, and understanding them is essential. High temperature behavior requires more concepts, and more complexity of analysis, but high temperature behavior is especially relevant to phase transformations in materials science and engineering.

Experimental facilities for inelastic scattering of neutrons and x-rays have made large advances in the past decade, and offer to make routine the acquisition of systematic data. Many of the barriers to high quality experimental results are now on the analysis side of the work, rather than on the instrumentation side, as has been the case historically. Calculations of vibrational entropy are fruitful today, and will get better as computational resources improve and new approaches become available. The strengths and weaknesses of experiment and theory can often be overcome by working with data and computation in the same computing environment, using 
the strengths of theory to support weaknesses in experimental measurements, and vice-versa.

Many important systematic trends on the vibrational thermodynamics of materials are now known. Nevertheless, relatively few materials have been studied to date, and there is much more to do. The situation has a parallel to the atomic structure of materials, which has been an active area of research for a hundred years. The vibrational motions of atoms in materials, and how they originate from structure and in turn influence the thermodynamic stability of the structure, will remain rich for discovery for years to come.

\section{Acknowledgments}

This work would not have been possible without the contributions of my junior collaborators at Caltech, especially L. Anthony, L.J. Nagel, H.N. Frase, A.F. Yue, M.E. Manley, P.D. Bogdanoff, J.Y.Y. Lin, T.L. Swan-Wood, A.B. Papandrew, O. Delaire, M.S. Lucas, M.G. Kresch, M.L. Winterrose, and J. Purewal, several of whom are taking this field into new directions. I acknowledge a debt to my collaborators in research, especially R. Nicklow, J.L. Robertson, R. Osborn, S. Rosenkranz, R. Ravelo, M. Baskes, R.J. McQueeney, W. Sturhahn, T.S. Toellner, E.E. Alp, I. Halevy, D. Abernathy, D.A. Neuman, L. Nazar, H-K. Mao. Software development and computational science were important in this work, and will be more so in the future. For this I acknowledge contributions from M.A.G. Aivazis, T.M. Kelley, M. McKerns, J.B. Keith. Important ideas have come from stimulating conversations over the years with A. van de Walle, V. Ozolins, G. Ceder, M. Asta, D.D. Johnson, D. de Fontaine, A.G. Khachaturyan, A. Zunger, and P. Rez. This work was supported by the DOE BES under grant DE-FG02-03ER46055.

\section{Bibliography}

\section{References}

1. J.W. Gibbs, Trans. Conn. Acad. 11, 382 (1873).

2. R. Kikuchi, Phys. Rev. 81, 988 (1951).

3. R. Kikuchi, J. Chem. Phys. 60, 1071 (1974).

4. D. de Fontaine, Solid State Physics 34, 73 (1979).

5. A.A. Maradudin, E. W. Montroll, G.H. Weiss, and I.P. Ipatova Theory of Lattice Dynamics in the Harmonic Approximation (Academic Press, New York, 1971).

6. M. Born and K. Wang, Dynamical Theory of Crystal Lattices (Oxford Classic Texts, Clarendon Press, Oxford, 1988).

7. O. Stern, Ann. Phys. (4 Folge), 51, 237 (1916).

8. F. Ducastelle, Order and Phase Stability in Alloys (North-Holland, Amsterdam, 1991).

9. A. van de Walle and G. Ceder, Revs. Mod. Phys. 74, 11 (2002).

10. J. Rosen and G. Grimvall, Phys. Rev. B, 27, 7199 (1983).

11. G.D. Garbulsky and G. Ceder, Phys. Rev. B 49, 6327 (1994).

12. R. Brout and W.M. Visscher, Phys. Rev. Lett. 9, 54 (1962).

13. Yu.M. Kagan and Ya.A. Iosilevskii, Zh. Eksp. Teor. Fiz. 42, 259 (1962). [English translation Sov. Phys. JETP 15, 182 (1962).] 
14. I.M. Lifshitz, Adv. Phys. 13, 483 (1964).

15. A.A. Maradudin, in Solid State Physics, F. Seitz and D. Turnbull, eds., 18, 273 (Academic Press, New York, 1966). Ibid. 19, 1 (1966).

16. A. Fernández Guillermet and G. Grimvall, Phys. Rev. B 40, 1521 (1989).

17. E.G. Moroni, G. Grimvall, and T. Jarlborg, Phys. Rev. Lett. 76, 2758 (1996).

18. V.L. Moruzzi, J.F. Janak, and K. Schwarz, Phys. Rev. B 37, 790 (1988).

19. J.M. Sanchez, J.P. Stark, and V.L. Moruzzi, Phys. Rev. B 44, 5411 (1991).

20. W.-T. Tseng and J.P. Stark, Philos. Mag. B 70, 919 (1990).

21. F.J. Dyson, Phys. Rev. 92, 1331 (1953).

22. P. Dean, Rev. Mod. Phys. 44, 127 (1972).

23. P.B. Allen and J. Kelner, Amer. J. Phys. 66, 497 (1998).

24. G. Moraitis and F. Gautier, J. Phys. F: Metal Phys. 7, 1421 (1977).

25. J.A.D. Matthew, R.E. Jones, and V.M. Dwyer, J. Phys. F: Metal Phys. 13, 581 (1983).

26. A.A.H.J. Waegemaekers and H. Bakker, Mat. Res. Soc. Symp. Proc. 21, 343 (1984).

27. G.D. Garbulsky and G. Ceder, Phys. Rev. B 53, 8993 (1996).

28. S. Baer, J. Phys. C: Solid State Phys. 16, 4103 (1983).

29. J. Mahanty and M. Sachdev, J. Phys. C 3, 773 (1970).

30. H. Bakker, Philos. Mag. A 45, 213 (1982).

31. H. Bakker, Phys. Stat. Solidi (b) 109, 211 (1982).

32. W.L. Bragg and E.J. Williams, Proc. Roy Soc. London A 151, 540 (1935).

33. B.E. Warren X-Ray Diffraction (Dover, Mineola New York, 1990).

34. Brent Fultz and James M. Howe, Transmission Electron Microscopy and Diffractometry of Materials Third Edition, (Springer-Verlag, Heidelberg 2007).

35. H. Bakker and C. Tuijn, J. Phys. C-Solid State 19, 5585 (1986).

36. C. Tuijn and H. Bakker, Phys. Stat, Solidi B 155, 107 (1989).

37. Cornelis Tuijn, Ph.D. Dissertation, van der Waals-Zeeman Institute, Univ. Amsterdam (1997).

38. G. Grimvall and A. Fernández Guillermet, Chapter 10 in Thermodynamic Data, edited by S.K. Saxena (Springer-Verlag 1992).

39. A. Fernández Guillermet and G. Grimvall, Phys. Rev. B 40, 10582 (1989).

40. G. Grimvall and A. Fernández Guillermet, Int. J. Thermophysics 15, 1353 (1994).

41. O.L. Anderson and J.E. Nafe, J. Geophys. Res. 70, 3951 (1965).

42. M.H.F. Sluiter, M. Weinert, and Y. Kawazoe, Phys. Rev. B 59, 4100 (1999).

43. A. van de Walle and G. Ceder, Phys. Rev. B 61, 5972 (2000).

44. E.J. Wu, G. Ceder, and A. van de Walle, Phys. Rev. B 67, 134103 (2003).

45. J.Z. Liu, G. Ghosh, A. van de Walle, and M. Asta, Phys. Rev. B 75, 104117 (2007).

46. D.C. Wallace, Thermodynamics of Crystals (Dover, Mineola, New York, 1998).

47. J. Trampenau, W. Petry, C. Herzig, Phys. Rev. B 47, 3132 (1993).

48. P.D. Bogdanoff, B. Fultz, J.L. Robertson and L. Crow, Phys. Rev. B 65, 014303 (2002).

49. M.E. Manley, R.J. McQueeney, B. Fultz, R. Osborn, G.H. Kwei, and P.D. Bogdanoff, Phys. Rev. B 65, 144111 (2002).

50. D.C. Wallace, Statistical Physics of Crystals and Liquids: A Guide to Highly Accurate Equations of State (World Scientific, Singapore, 2003).

51. C. Triguero, M. Porta, and A. Planes, Phys. Rev. B 73, 054401 (2006).

52. P. Entel, E. Hoffmann, P. Mohn, K. Schwarz, and V. L. Moruzzi, Phys. Rev. B 47, 8706 (1993).

53. Y. Wang, G. M. Stocks, D. M. C. Nicholson. W. A. Shelton, V. P. Antropov, and B. N. Harmon, J. Appl. Phys. 81, 3873 (1997).

54. M. van Schilfgaarde, I. A. Abrikosov, and B. Johansson, Nature (London) 400, 46 (1999).

55. W. Nernst and F.A. Lindemann, Berl. Ber. 494, (1911).

56. E.S.R. Gopal, Specific Heats at Low Temperatures, (Plenum, New York, 1966).

57. J.P. McCullough and D.W. Scott, eds. Experimental Thermodynamics Volume 1 Calorimetry of Non-reacting systems (Plenum, New York, 1968).

58. G.K. White, Experimental Techniques in Low-Temperature Physics (Oxford, 1979).

59. R. Stevens, J. Boerio-Goates, J. Chem. Thermodynamics 36, 857 (2004). 
60. B.E. Lang, J. Boerio-Goates and B.F. Woodfield, J. Chem. Thermodynamics 38, 1655 (2006).

61. R. Bachmann, et al., Rev. Sci. Instr. 43, 205 (1972).

62. G.R. Stewart, Rev. Sci. Instr. 54, 1 (1983).

63. J.C. Lashley, et al. Cryogenics 43, 369 (2003).

64. C.A. Kennedy, et al., Cryogenics 47107 (2007).

65. P.F. Sullivan and G. Seidel, Phys. Rev. 173, 679 (1968).

66. L. Van Hove, Phys. Rev. 95, 249 (1954).

67. V.S. Oskotskii, Fiz. Tverdogo Tela 9, 550 (1967). [English translation Sov. Phys. - Solid State 9, 420 (1967).]

68. M.M. Bredov, B.A. Kotov, N.M. Okuneva, V.S. Oskotskii, and A.L. Shakh-Budagov Fiz. Tverdogo Tela 9, 287 (1967). [English translation Sov. Phys. - Solid State 9, 214 (1967).]

69. F.W. de Wette and A. Rahman, Phys. Rev. 176, 784 (1968).

70. V. Ambegaokar, J.M. Conway, and G. Baym, in Lattice Dynamics, R.F. Wallis Ed., (Pergamon Press, Oxford, 1965) 261.

71. R.A. Cowley and W.J.L. Buyers, J. Phys. C: Solid State 2, 2262 (1969).

72. W.J.L. Buyers and R.A. Cowley, Phys. Rev. 180, 755 (1969).

73. T. May, W. Müller and D. Strauch, Phys. Rev. B 575758 (1998).

74. C.G. Windsor, Pulsed Neutron Scattering (Taylor \& Francis, London 1981).

75. http://neutrons.ornl.gov/instrument_systems/sns_instruments.shtml

76. http://www.isis.rl.ac.uk/instruments/index.htm

77. http://lansce.lanl.gov/lujan/instruments/Pharos/index.html

78. http://j-parc.jp/index-e.html

79. B.N. Brockhouse, Phys. Rev. 98, 1171 (1955).

80. A.D.B. Woods, W. Cochran, and B.N. Brockhouse, Phys Rev. 119, 980 (1960).

81. G. Shirane, S.M. Shapiro, and J.M. Tranquada, Neutron Scattering with a Triple-Axis Spectrometer (Cambridge, 2002).

82. R.M. Martin, Electronic Structure (Basic Theory and Practical Methods) (Cambridge, 2005).

83. C. Fiolhais, Fernando Nogueira, Miguel A.L. Marques, Editors, A Primer in Density Functional Theory (Lecture Notes in Physics) (Springer-Verlag, Berlin 2003).

84. M. Finnis, Interatomic Forces in Condensed Matter (Oxford Series on Materials Modelling, 1) (Cambridge, 2004).

85. J. Kohanoff, Electronic Structure Calculations for Solids and Molecules: Theory and Computational Methods (Cambridge University Press, 2006).

86. S. Baroni, P. Giannozzi, and A. Testa, Phys. Rev. Lett 58, 1861 (1987).

87. S. Baroni, S. de Gironcoli, and P. Giannozzi, Phys. Rev. Lett 65, 84 (1990).

88. S. Wei and M.Y Chou, Phys. Rev. Lett. 69, 2799 (1992).

89. X. Gonze, Phys. Rev. B 55, 10337 (1997).

90. X. Gonze and C. Lee, Phys. Rev. B 55, 10355 (1997).

91. K. Parlinski, Z.Q. Li, Y. Kawazoe, Phys. Rev. Lett 784063 (1997).

92. G.J. Ackland, M.C. Warren, and S.J. Clark, J. Phys.: Condens. Matter 9, 7861 (1997).

93. X. Gonze, G.M. Rignanese, R. Caracas, Z. Krist. 220, 458 (2005).

94. http://www.abinit.org/

95. P. Hohenberg and W. Kohn, Phys. Rev. 136, B864 (1964).

96. N.D. Mermin, Phys. Rev. 137, A1441 (1965).

97. W. Kohn and L.J. Sham, Phys. Rev. 140, A1133 (1965).

98. J.M. Ziman, Electrons and Phonons (Oxford, 1963).

99. Peter Bruesch, Phonons: Theory and Experiments One (Springer, 1982).

100. M.T. Yin and M.L. Cohen, Phys. Rev. Lett. 451004 (1980).

101. K. Kunc and R.M. Martin, Phys. Rev. B 24, 2311 (1981).

102. J. Zaretsky, C. Stassis, B.N. Harmon, K.-M. Ho, and C.-L. Fu, Phys. Rev. B 28, 697 (1983).

103. Y.-Y. Ye, Y. Chen, K.-M. Ho and B.N. Harmon, Phys. Rev. Lett. 58, 1769 (1987).

104. Y.-Y. Ye, Y. Chen, K.-M. Ho and B.N. Harmon, J. Phys.: Condens. Matter 3, 9629 (1991).

105. A.A. Quong and B.M. Klein, Phys. Rev. B 46, 10734 (1992).

106. S. Nosé, J. Chem. Phys. 81, 511 (1984). 
107. W.G. Hoover, A.J.C. Ladd, and B. Moran, Phys. Rev. Lett. 48, 1818 (1982).

108. J.M. Dickey and A. Paskin, Phys. Rev. 188, 1407 (1969).

109. R. Car and M. Parrinello, Phys. Rev. Lett. 55, 2471 (1985).

110. T.D. Kuhne, M. Krack, F.R. Mohamed and M. Parrinello, Phys. Rev. Lett. 98, 066401 (2007).

111. Slater, J.C. (1939) Introduction to Chemical Physics, McGraw-Hill, New York, Chapter 13.

112. F. Seitz, The Modern Theoery of Solids (McGraw-Hill, New York, 1940) Chapter 14.

113. F. Lange, Z. Physik. Chem. 30A, 601 (1924).

114. C. Zener, Elasticity and Anelasticity of Metals (University of Chicago Press, Chicago, 1948).

115. G. Grimvall and I. Ebbsjö, Physica Scripta 12, 168 (1975).

116. A.O.E. Animalu, Phys. Rev. 161, 445 (1967).

117. R. Pynn and I. Ebbsjö, J. Phys. F: Metal Phys. 1, 744 (1971).

118. D.C. Wallace, Phys. Rev. 176, 832 (1968).

119. F. Frey, W. Prandl, C. Zeyen, and K. Ziebeck, J. Phys. F: Maetal Phys. 9, 603 (1979).

120. L.J. Nagel, B. Fultz, and J.L. Robertson, Philos. Mag. B 75, 681 (1997).

121. J.L. Robertson, B. Fultz, and H.N. Frase, Phys. Rev. B 60, 9329 (1999).

122. R. Lübbers, H.F. Grünsteudel, A.I. Chumakov, and G. Wortmann, Science 287, 1250 (2000).

123. H.-K. Mao, V. Struzhkin, J. Shu, R.J. Hemley, W. Sturhahn, M.Y. Hu, E.E. Alp, L. Vocadlo, D. Alfe, G.D. Price, M.J. Gillan, M. Schwoerer-Böhning, D. Häusermann, P. Eng, G. Shen, H. Geifers, R. Lubers, G. Wortmann, Science 292, 914 (2001).

124. G. Shen, W. Sturhahn, E.E. Alp, J. Zhao, T.S. Toellner, V.B. Prakapenka, Y. Meng, and H.-K. Mao, Phys. Chem. Minerals 31, 353 (2004).

125. A. van de Walle, A. Moser, and W. Gasior Archives of Metallurgy and Materials, 49, 535 (2004).

126. C. Zener, J. Appl. Phys. 22, 372 (1951).

127. G.H. Vineyard, J. Phys. Chem. Solids 3, 121 (1957).

128. H.R. Schoeber, W. Petry and J. Trampenau, J. Phys. Condens. Matter 4, 9321 (1992).

129. C. Zener, in Thermodynamics in Physical Metallurgy, C. Zener ed., (ASM, Cleveland, 1950) p. 16.

130. H.B. Huntington, G.A. Shirn and E.S. Wajda, Phys. Rev. 99, 1085 (1955).

131. J.F. Freedman and A.S. Nowick, Acta Metal. 6, 176 (1956).

132. W. Desorbo, Acta Metall. 3, 227 (1955).

133. R.A. Oriani, Acta Metall. 3, 232 (1955).

134. A. Fernández Guillermet and G. Grimvall, J. Phys. Chem. Solids 53, 105 (1991).

135. A. Fernández Guillermet and H. Du, Z. Metallkunde 85, 154 (1994).

136. A. Seko, F. Oba, A. Kuwabara, and I. Tanaka, Phys. Rev. B 72, 024107 (2005).

137. A.P. Giddy, M.T. Dove, G.S. Pawley, and V. Heine Acta Crystallogr. A 49, 697 (1993).

138. M.T. Dove, Introduction to Lattice Dynamics, Cambridge Topics in Mineral Physics and Chemistry (Cambridge Univ. Press, 1993).

139. G. Ernst, C. Broholm, G.R. Kowach, and A.P. Ramirez, Nature 396, 147 (1998).

140. R. Mittal, S.L. Chaplot, H. Schober, and T.A. Mary, Phys. Rev. Lett 86, 4692 (2001).

141. C.A. Kennedy, M.A. White, A.P. Wilkinson, T. Varga, Phys. Rev. B 75, 224302 (2007).

142. M. Blackman, Proc. Phys. Soc. B 70, 827 (1957).

143. G.B. Walker, Phys. Rev. 103, 547 (1956).

144. R. Stedman and G. Nilsson, Phys. Rev. Lett. 15, 634 (1965).

145. G. Gilat and R. M. Nicklow, Phys. Rev. 143, 487 (1965).

146. V. Ozolins and M. Asta, Phys. Rev. Lett. 86, 448 (2001).

147. M. Asta and V. Ozolins, Phys. Rev. B 64, 094104 (2001).

148. V. Ozolins, B. Sadigh, and M. Asta, J. Phys.: Condens. Matter 17, 2197 (2005).

149. J. Chevrier, J.B. Suck, J.C. Lasjaunias, M. Perroux, and J.J. Capponi, Phys. Rev. B 49, 961 (1994).

150. C. Wolverton and V. Ozolins, Phys. Rev. Lett. 86, 5518 (2001).

151. M. Mihalkovic and M. Widom, Phys. Rev. B 75, 014207 (2007).

152. H. Okamoto, Phase Diagrams for Binary Alloys (Desk Handbook), (ASM International, Materials Park, OH, 2000). 
153. W. Hume-Rothery and G.V. Raynor, The Structure of Metals and Alloys (Institute of Metals, London, 1962).

154. A. Gangulee, G.C. Das, and M.B. Bever, Metall. Trans. 4, 2063 (1973).

155. Fong-Chi Hsu, Jiu-Yong Luo, Kuo-Wei Yeh, Ta-Kun Chen, Tzu-Wen Huang, Phillip M. Wu, Yong-Chi Lee, Yi-Lin Huang, Yan-Yi Chu, Der-Chung Yan, Maw-Kuen Wu, "Superconductivity in the PbO-type Structure alpha-FeSe," arXiv:0807.2369.

156. A. Subedi, L. Zhang, D.J. Singh, and H.M. Du, Phys. Rev. B 78, 134514 (2008).

157. H. Ikeda, M. Shirai, N. Suzuki, and K. Motizuki, Jpn. J. Appl. Phys. 32 Suppl. 32-3, 301 (1993).

158. E.M. Carvaljo, I.R. Harris, J. Mater. Sci. 15, 1224 (1980).

159. E. Kneller, Y. Khan, and U. Gorres, Z. Metallkunde 77, 43 (1986).

160. K.-J. Zeng, M. Hämäläinen, and H.L. Lukaz, J. Phase Equilibria 15, 577 (1994).

161. J. Okamoto, C. Ahn, and B. Fultz, in Microbeam Analysis - 1990, J.R. Michael and P. Ingram, eds. (San Francisco Press, 1990) p. 56.

162. L.J. Nagel, L. Anthony, J. K. Okamoto, and B. Fultz, Journal of Phase Equilibria 18, 551 (1997).

163. L. Anthony, L. J. Nagel, J. K. Okamoto, and B. Fultz, Phys. Rev. Lett. 73, 3034 (1994).

164. James Kozo Okamoto, Ph.D. Thesis in Applied Physics, California Institute of Technology, May 6, 1993.

165. B. Fultz, W. Sturhahn, T.S. Toellner, and E.E. Alp, in MRS Symposium Proceedings 590, Applications of Synchrotron Radiation Techniques to Materials Science V, S.R. Stock, S.M. Mini, and D.L. Perry, eds. (Materials Research Society, Warrrendale, PA 2000) p. 91.

166. B. Fultz, T.A. Stephens, W. Sturhahn, T.S. Toellner, and E.E. Alp, Phys. Rev. Lett. 80, 3304 (1998).

167. L. Anthony, J. K. Okamoto, and B. Fultz, Phys. Rev. Lett. 70, 1128 (1993).

168. L. Anthony, L. J. Nagel, and B. Fultz, in Solid-Solid Phase Transformations, W.C. Johnson, J. Howe, D.E. Laughlin, and W.A. Soffa, eds. (TMS, Warrendale, 1994) 467.

169. B. Fultz, L. Anthony, L.J. Nagel, R.M. Nicklow and S. Spooner, Phys. Rev. B 52, 3315 (1995).

170. C. Stassis, F.X. Kayser, C.-K. Loong, and D. Arch, Phys Rev. B 24, 3048 (1981).

171. J.D. Althoff, D. Morgan, D. de Fontaine, M. Asta, S.M. Foiles, and D.D. Johnson, Phys. Rev. B 56, R5705 (1997).

172. R. Ravelo, J. Aguilar, M. Baskes, J.E. Angelo, B. Fultz, and B.L. Holian, Phys. Rev. B 57, 862 (1998).

173. J.D. Althoff, D. Morgan, D. de Fontaine, M. Asta, S.M. Foiles, and D.D. Johnson, Comp. Mater. Sci. 10, 411 (1998).

174. D. Morgan, J.D. Althoff and D. de Fontaine, J. Phase Equilibria 19, 559 (1998).

175. S.J. Liu, S.Q. Duan, and B.K. Ma, Phys. Rev. B 58, 9705 (1998).

176. A. van de Walle, G. Ceder, and U.V. Waghmare, Phys. Rev. Lett. 80, 4911 (1998).

177. W.L. Bragg and E.J. Williams, Proc. Roy. Soc. A 145, 699 (1934).

178. H.A. Bethe Proc. Roy. Soc. A, 150, 552 (1935).

179. F.C. Nix and W. Shockley, Rev. Mod. Phys. 10, 1 (1938).

180. C. Zener, Phys. Rev. 71, 846 (1947).

181. L. Saroléa, Physica 19, 615 (1953).

182. C. Booth and J.S. Rowlinson Trans. Faraday Soc. 51, 463 (1955).

183. P.J. Wojtowciz and J.G. Kirkwood, J. Chem. Phys. 33, 1299 (1960).

184. Douglas Harvey Pearson, Ph.D. Thesis in Applied Physics, California Institute of Technology, September 30, 1991.

185. A.S. Normanton, P.E. Bloomfield, F.R. Sale, and B.B. Argent, Metal Science 9510 (1975).

186. T. Eguchi, H. Matsuda and K. Oki, IEEE Trans. Magnetics Mag-4, 476 (1968).

187. H.H. Hamdeh, J. Okamoto, and B. Fultz, Phys. Rev. B 42, 6694 (1990).

188. Matthew S. Lucas, Ph.D. Thesis in Materials Science, California Institute of Technology, July 25, 2008.

189. D.W. Clegg and R.A. Buckley, Metal Science 7, 48 (1973).

190. D.B. Bowen, Acta Metall. 2, 573 (1954). 
191. P.A. Flinn, G.M. McManus and J.A. Rayne, J. Phys. Chem. Solids 15, 189 (1960).

192. P.L. Rossiter and B. Bykovec, Philos. Mag. B 38, 555 (1978).

193. J. Rayne, Phys. Rev. 108, 649 (1957).

194. S. Katano, M. Iizumi, Y. Noda, J. Phys. F. 18, 2195 (1988).

195. L.J. Nagel, L. Anthony and B. Fultz, Philos. Mag. Lett. 72, 421 (1995).

196. Laura Jeanne Nagel, Ph.D. in Materials Science, California Institute of Technology, June 18, 1996.

197. F. Cleri and V. Rosato, Philos. Mag. Lett. 67, 369 (1993).

198. G.J. Ackland, in Alloy Modeling and Design, G. M. Stocks and P. Turchi, eds. (The Metals, Minerals and Materials Society, Warrendale, PA 1994) 149.

199. W. B. Fei, A. Kara, T. S. Rahman, Phys. Rev. B 61, 16105 (2000).

200. P. D. Bogdanoff, B. Fultz, and S. Rosenkranz, Phys. Rev. B 60, 3976 (1999).

201. P.D. Bogdanoff, T. Swan-Wood, and B. Fultz, Phys. Rev. B 68, 014301 (2003).

202. V. Ozolins, C. Wolverton and A. Zunger, Phys. Rev. B 58, R5897 (1998).

203. R.J. Elliott and D.W. Taylor, Proc. R. Soc. London, Ser. A 296, 161 (1967).

204. B. Fultz, T.A. Stephens, E.E. Alp, M.Y. Hu, J.P. Sutter, T.S. Toellner, and W. Sturhahn, Phys. Rev. B 61, 14517 (2000).

205. Y. Noda and Y. Endoh, J. Phys. Soc. Japan 57, 4225 (1988).

206. P.D. Bogdanoff and B. Fultz, Philos. Mag. B 79, 753 (1999).

207. A. F. Yue, I. Halevy, A. Papandrew, P. D. Bogdanoff, B. Fultz, W. Sturhahn, E. E. Alp, and T. S. Toellner, Hyperfine Interact. 141, 249 (2002).

208. W.G. Stirling, R.A. Cowley and M.W. Stringfellow, J. Phys. F: Metal Phys. 2, 421 (1972).

209. V. Ozolins, C. Wolverton, and A. Zunger, Phys. Rev. B 57, 6427 (1998).

210. P.J. Craievich and J.M. Sanchez, Computational Materials Science 8, 92 (1997).

211. N.A. Zarkevich, T.L. Tan, and D.D. Johnson, Phys. Rev. B 75, 104203 (2007).

212. B.P. Burton, A. van de Walle and U. Kattner, J. App. Phys. 100, 113528 (2006).

213. H.Y. Genga, M.H.F Sluiter, and N.X. Chen, J. Chem. Phys. 122, 214706 (2005).

214. J.W.D. Connolly and A.R. Williams, Phys, Rev. B 27, 5169 (1983).

215. M.S. Lucas, M. Kresch, R. Stevens, and B. Fultz, Phys. Rev. B 77, 184303 (2008).

216. M.S. Lucas, A. Papandrew, B. Fultz and M.Y. Hu, Phys. Rev. B 75, 054307 (2007).

217. T. Chart, F. Putland, and A. Dinsdale, CALPHAD: Comput. Coupling Phase Diagrams Thermochem. 4, 27 (1980).

218. A. Caro, M. Caro, E.M. Lapasso, and D.A. Crowson, Appl. Phys. Lett. 89, 121902 (2006).

219. P. Olsson, I.A. Abrikosov, L. Vitos, and J. Wallenius, J. Nucl. Mater. 321, 84 (2003).

220. E.G. Moroni and T. Jarlborg, Phys. Rev. B 47, 3255 (1993).

221. S.A. Danilkin, M.G. Zemlyanov, V.P. Minaev, P.P. Parshin, and V.V. Sumin, Fiz. Tverd. Tela (Leningrad) 29, 2122 (1987). [English translation Sov. Phys. Solid State 29, 1213 (1987).]

222. S.A. Danilkin, M.G. Zemlyanov, V.P. Minaev, P.P. Parshin, and V.V. Sumin, Fiz. Tverd. Tela (Leningrad) 31, 8 (1989). [English translation Sov. Phys. Solid State 31, 725 (1989).]

223. S.A. Danilkin, V.P. Minaev, and V.V. Sumin, Physica B 174, 241 (1991).

224. W. Luo, J.D. Clewley and T.B. Flanagan, J. Chem. Phys. 93, 6710 (1990).

225. G. Grimvall, J. Alloys and Compounds 233, 183 (1996).

226. J.L. Anderson, J. Nasise, K. Philipson, and F.E. Pretzel, J. Phys. Chem. Solids 31, 613 (1970).

227. W. Yu, C. Jin, and A. Kohlmeyer, J. Phys.: Condens. Matter 19, 086209 (2007).

228. B. Yates, G.H. Wostenholm, and J.K. Bingham, J. Phys. C: Solid State Phys. 7, 1769 (1974).

229. V. Ozolins, E.H. Majzoub, and C. Wolverton, J. Am. Chem. Soc. (2009).

230. R. Moreh, N. Shnieg, H. Zabel, Phys. Rev. B 44, 13111 (1994).

231. M.M. Beg, M. Nielsen, Phys. Rev. B 14, 4266 (1976).

232. Y. Reynier, R. Yazami, and B. Fultz, J. Power Sources 119-121, 850 (2003).

233. Y. Reynier, J. Graetz, T. Swan-Wood, P. Rez, R. Yazami and B. Fultz, Phys. Rev. B 70, 174304 (2004).

234. G.V. Kurdjumov and A.G. Khachaturyan, Metall Trans. 3, 1069 (1972).

235. P.C. Chen, B.O. Hall, and P.G. Winchell, Metall Trans. A 11, 1323 (1980).

236. K.A. Taylor and M. Cohen, Prog. Mater. Sci. 36, 225 (1992). 
237. B.D. Butler and B. Cohen, Ultramicroscopy 52, 238 (1993).

238. J.R. Patel and M. Cohen, Acta Metall. 1, 531 (1953).

239. C. Zener, Trans. AIME 203, 619 (1955).

240. L. Kaufman and M. Cohen, Prog. Mater. Physics 7, 165 (1958).

241. T. Bell and W.S. Owen, Trans. AIME 239, 1940 (1967).

242. H.C. Tong and C.M. Wayman, Acta Metall. 23, 209 (1975).

243. A. Borgenstam and M. Hillert Acta Mater. 45, 2079 (1997).

244. Q. Chen and B. Sundman, J. Phase Equilibria 22, 631 (2001).

245. E.S. Scheil, Anorg. Allg. Chem. 207, 21 (1932).

246. P.C. Clapp Phys. Stat. Sol. b 57, 561 (1973).

247. J. Friedel, J. Phys. Lett (Paris) 35, 59 (1974).

248. J.W. Cahn, Prog. Materials Sci. 36, 149 (1992).

249. Y. Murakami, J. Phys. Soc. Jpn. 33, 1350 (1972).

250. L. Mañosa, A. Planes, J. Ortín and B. Martínez, Phys. Rev. B 45, 7633 (1992).

251. L. Mañosa, A. Planes, J. Ortín and B. Martínez, Phys. Rev. B 48, 3611 (1993).

252. E. Obradó, L. Mañosa, and A. Planes, Phys. Rev. B 56, 20 (1997).

253. P. Bogdanoff and B. Fultz, Philos. Mag. B 81, 299 (2001).

254. G. Herget, Müllner, J.B. Suck, R. Schmidt, and H. Wipf, Europhys. Lett. 10, 49 (1989).

255. O. Delaire, M. Kresch, and B. Fultz, Philos. Mag. 85, 3567 (2005).

256. H. Franz, T. Asthalter, M. Dommach, A. Ehnes, K. Messel and I. Sergueev, Hyperfine Interact. 141/142, 131 (2002).

257. A. Zheludev, S.M. Shapiro, P. Wochner, and L.E. Tanner, Phys. Rev. B 54, 15045 (1996).

258. C.P. Opeil, B. Mihaila, R.K. Schulze, L. Manosa, A. Planes, W.L. Hults, R.A. Fisher, P.S. Riseborough, P.B. Littlewood, J.L. Smith, and J.C. Lashley, Phys. Rev. Lett. 100, 165703 (2008).

259. M.A. Uijttewaal, T. Hickel, J. Neugebauer, M.E. Gruner, and P. Entel, Phys. Rev. Lett. 102, 035702 (2009).

260. L.J. Nagel, B. Fultz, J.L. Robertson, and S. Spooner, Phys. Rev. B 55, 2903 (1997).

261. M.E. Manley, B. Fultz, and L.J. Nagel, Philos. Mag. B 80, 1167 (2000).

262. M.E. Manley, B. Fultz, D.W. Brown, B. Clausen, A.C. Lawson, J.C. Cooley, W.L. Hults, R.J. Hanrahan, Jr., J.L. Smith, and D.J. Thoma, Phys. Rev. B 66, 024117 (2002).

263. A. Tschöpe and R. Birringer, Acta Metall. Mater. 41, 2791 (1993).

264. L. B. Hong and B. Fultz, J. Appl. Phys. 79, 3946 (1996).

265. B. Fultz, H. Kuwano, and H. Ouyang, J. Appl. Phys. 77, 3458 (1995).

266. W.D. Kristensen, E.J. Jensen, and R. M. Cotterill, J. Chem. Phys. 60, 4161 (1974).

267. A. Tamura, H. Higeta, and T. Ichinokawa, J. Phys. C, 15, 4975 (1982).

268. A. Tamura, T. Ichinokawa, J. Phys. C, 16, 4779 (1983).

269. A. Tamura, H. Higeta, and T. Ichinokawa, J. Phys. C, 16, 1585 (1983).

270. J. Wang, D. Wolf, and S.R. Philpot, Philos. Mag A 73, 517 (1996).

271. K. Suzuki and K. Sumiyama, Mater. Trans. JIM 36, 188 (1995).

272. J. Trampenau, K. Bauszuz, W. Petry, and U. Herr, Nanostruct. Mater. 6, 551 (1995).

273. B. Fultz, J.L. Robertson, T.A. Stephens, L.J. Nagel, and S. Spooner, J. Appl. Phys. 79, 8318 (1996).

274. H.N. Frase, L. J. Nagel, J.L. Robertson and B. Fultz, Philos. Mag. B 75, 335 (1997).

275. H. Frase, B. Fultz and J.L. Robertson, Phys. Rev. B 57, 898 (1998).

276. A.B. Papandrew, A.F. Yue, B. Fultz, I. Halevy, W. Sturhahn, T.S. Toellner, E.E. Alp, and H-K. Mao, Phys. Rev. B 69, 144301 (2004).

277. B. Fultz, C.C. Ahn, E.E. Alp, W. Sturhahn, T.S. Toellner, Phys. Rev. Lett. 79, 937 (1997).

278. E. Bonetti, L. Pasquini, and E. Sampaolesi, A. Deriu, and G. Cicognani, J. Appl. Phys. 88, 4571 (2000).

279. A.F. Yue, A.B. Papandrew, O. Delaire, B. Fultz, Z. Chowdhuri, R. Dimeo and D.A. Neumann, Phys. Rev. Lett. 93, 205501 (2004).

280. A. Kara and T.S. Rahman, Phys. Rev. Lett. 81, 1453 (1998).

281. U. Stuhr, H. Wipf, K.H. Anderson, and H. Hahn, Phys. Rev. Lett. 81, 1449 (1998). 
282. P.M. Derlet, R. Meyer, L.J. Lewis, U. Stuhr, and H. van Swygenhoven, Phys. Rev. Lett. 87, 205501 (2001).

283. B. Roldan Cuenya, A. Naitabdi, J. Croy, W. Sturhahn, J. Y. Zhao, E.E. Alp, R. Meyer, D. Sudfeld, E. Schuster, and W. Keune, Phys. Rev. B 76, 195422 (2007).

284. B. Roldan Cuenya, W. Keune, R. Peters, E. Schuster, B. Sahoo, U. von Hörsten, W. Sturhahn, J. Zhao, T.S. Toellner, E.E. Alp and S.D. Bader, Phys. Rev. B 77, 165410 (2008).

285. H.N. Frase, L.J. Nagel, J.L. Robertson, and B. Fultz, in E. Ma, B. Fultz, R. Shull, J. Morral, and P. Nash, eds., Chemistry and Physics of Nanostructures and Related Non-Equilibrium Materials, (TMS, Warrendale, PA, 1997), ISBN No. 0-87339-358-8. p. 125.

286. H.N. Frase, B. Fultz, S. Spooner, and J.L. Robertson, J. Appl. Phys. 85, 7097 (1999).

287. H.N. Frase, B. Fultz, J. L. Robertson and S. Spooner, Philos. Mag. B 80, 1545 (2000).

288. B. Fultz and H. N. Frase, in Ultrafine Grained Materials, R. S. Mishra, S. L. Semiatin, C. Suryanarayana N.N. Thadhani, and T. C. Lowe, eds., (TMS, Warrendale PA, 2000) p. 3.

289. B. Fultz and H. N. Frase, Hyperfine Interact. 130, 81 (2000).

290. D. Wolf, J. Wang, S. R. Phillpot, and H. Gleiter, Phys. Rev. Lett. 74, 4686 (1995).

291. J.L. Robertson, H.N. Frase, P.D. Bogdanoff, M.E. Manley, and B. Fultz, Philos. Mag. Lett. 79, 297 (1999).

292. M.E. Manley, R.J. McQueeney, J.L. Robertson, B. Fultz, and D.A. Neumann, Philos. Mag. Lett. 80, 591 (2000).

293. G. Beggerow, in Landolt-Börnstein, New Series, Numerical Data and Functional Relationships in Science and Technology, K.H. Hellwege, (Ed.), Group IV, Vol. 4 (Springer-Verlag, Berlin, 1980) p. 9.

294. I. Barhin and G. Platzki, Thermochemical Data of Pure Substances, Vol. I (VCH, Weinheim, 1995) p. 507.

295. K.A. Gschneidner, Jr., V.K. Pecharsky, J. Cho, and S.W. Martin, Scr. Mater. 34, 1717 (1996).

296. M.E. Manley, R.J. McQueeney, B. Fultz, R. Osborn, G.H. Kwei, and P.D. Bogdanoff, Phys. Rev. B. 65, 144111 (2002).

297. R.J. McQueeney, M.E. Manley, R. Osborn, B. Fultz, G.H. Kwei, and P.D. Bogdanoff, Philos. Mag. B 81, 675 (2002).

298. M.E. Manley, R.J. McQueeney, B. Fultz, T. Swan-Wood, O. Delaire, E.A. Gorymychkin, J.C. Cooley, W.L. Hults, J.C. Lashley, R. Osborn, and J.L. Smith, Phys. Rev. B 67, 014103 (2003).

299. K.A. Gschneidner, Jr., R.O. Elliott, and R.R. McDonald, J. Phys. Chem. Solids 23, 1191 (1962).

300. I.-K. Jeong, T.W. Darling, M.J. Graf, Th. Proffen, R.H. Heffner, Y. Lee, T. Vogt, and J.D. Jorgensen, Phys. Rev. Lett. 92, 105702 (2004).

301. I.-K. Jeong, T.W. Darling, R.H. Heffner, A. Llobet, and J.C. Lashley, Philos. Mag. Lett. 88, 607 (2008).

302. J.C. Marmeggi and A. Delapalme, Physica (Amsterdam) 120B, 309 (1980).

303. G.H. Lander, E.S. Fisher, and S.D. Bader, Adv. Phys. 43, 1 (1994).

304. M.E. Manley, B. Fultz, R.J. McQueeney, C.M. Brown, W.L. Hults, J.L. Smith, D.J. Thomas, R. Osborn, and J.L. Robertson, Phys. Rev. Lett. 86, 3076 (2001).

305. P. Söderlind, O. Eriksson, B. Johansson, J. M. Wills, and A. M. Boring, Nature (London) 374, 524 (1995).

306. K.T. Moore and G. van der Laan, Rev. Modern Phys. 81, 235 (2009).

307. J. Wong, M. Krisch, D.L. Farber, F. Occelli, A.J. Schwartz, T.C. Chiang, M. Wall, C. Boro, and X.Q. Xu, Science 301, 1078 (2003).

308. R.J. McQueeney, A.C. Lawson, A. Migliori, T.M. Kelley, B. Fultz, M. Ramos, B. Martinez, J.C. Lashley, and S.C. Vogel, Phys. Rev. Lett 92, 146401 (2004).

309. X. Dai, et al., Science 300, 953 (2003).

310. W.H. Zachariasen, in The Metal Plutonium, A.S. Coffinberry and W.N. Miner, eds., (Univ. Chicago Press, Chicago, 1961) p. 99.

311. D.C. Wallace, Phys. Rev. B 58, 15433 (1998).

312. P.A.M. Dirac, The Principles of Quantum Mechanics (Oxford, Clarendon Press, 1958). 
313. N.W. Ashcroft and N.D. Mermin, Solid State Physics (Holt, Rinehart and Winston New York, 1976) Appendix L.

314. P.G. Klemens, Phys. Rev. 148, 845 (1966).

315. M. Balkanski, R.F. Wallis, and E. Haro, Phys. Rev. B 28, 1928 (1983).

316. G. Morell, W. Perez, E. Ching-Prado, and R.S. Katiyar, Phys. Rev. B, 53, 5388 (1996).

317. L. Chen, M. McKerns, and B. Fultz, Phys. Rev. B, in press (2009).

318. R.C. Shukla and E.R. Cowley, Phys. Rev. B 3, 4055 (1971). Ibid. 58, 2596 (1998). Ibid. 62, 3232 (2000).

319. S.W. Lovesey, Condensed Matter Physics: Dynamic Correlations Second Edition (Benjamin/Cummings, Menlo Park, CA, 1986) Chapter 1.

320. G.K. Horton and E.R. Cowley, Chaos 133, 667 (2003).

321. L. Van Hove, M. N. Hugenholz, and L.P. Howland, Quantum Theory of Many-Particle Systems (Benjamin, New York, 1961).

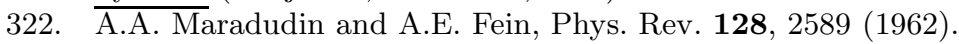

323. R.A. Cowley, Adv. Phys. 12, 421 (1963).

324. R.A. Cowley, Repts. Prog. Phys. 31, 123 (1968).

325. R.C. Shukla and E.R. Cowley, Phys. Rev. B 31, 372 (1985).

326. E.R. Cowley and G.K. Horton, Phys. Rev. Lett. 58, 789 (1987).

327. R.P. Lowndes, Phys. Rev. B 6, 1490 (1972).

328. P. Derollez, J. Gonzalez, B. Hennion, R. Fouret, Physica B 4 305, 191 (2001).

329. K. Schmalzl, D. Strauch, and H. Schober, Phys. Rev. B 68, 144301 (2003).

330. S. Ves, I. Loa, K. Syassen, F. Widulle and M. Cardona, Phys. Stat. Solidi (b), 223, 241 (2001).

331. M. Cardona, High Pressure Research 24, 17 (2004).

332. O. Anderson, Equations of State of Solids for Geophysicists and Ceramic Science (Oxford University Press, New York, 1995).

333. O.G. Randl, G. Vogl, W. Petry, B. Hennion, B. Sepiol, K. Nembach, J. Phys.: Condens. Matter 7, 5983 (1995).

334. O. Eriksson, J.M. Wills, and D. Wallace, Phys. Rev. B 46, 5221 (1992).

335. G. Grimvall, M. Thiessen, and A. Fernández Guillermet, Phys. Rev. B 36, 7816 (1987).

336. A. Fernández Guillermet and G. Grimvall, Phys. Rev. B 44, 4332 (1991).

337. C. Stassis, J. Zaretsky, D. Arch, O.D. McMasters and B.N. Harmon, Phys. Rev. B 18, 2632 (1978).

338. C. Stassis, G.S. Smith, B.N. Harmon, K.-M. Ho and Y. Chen, Phys. Rev. B 19, 181 (1979).

339. W. Petry, A. Heiming, J. Trampenau, M. Alba, C. Herzig, H.R. Schober, and G. Vogl, Phys. Rev. B 43, 10933 (1991).

340. A. Heiming, W. Petry, J. Trampenau, M. Alba, C. Herzig, H.R. Schober, and G. Vogl, Phys. Rev. B 43, 10948 (1991).

341. M. Kresch, O. Delaire, R. Stevens, J.Y.Y. Lin, and B. Fultz, Phys. Rev. B 75, 104301 (2007).

342. M. Kresch, M. Lucas, and B. Fultz, Phys. Rev. B 77, 024301 (2008).

343. M.G. Kresch, Ph.D. Thesis in Materials Science, California Institute of Technology, Dec. 5, 2008.

344. O. Delaire, M.G. Kresch, J. Munoz, M.S. Lucas, J.Y.Y. Lin, and B. Fultz, Phys. Rev. B 77, 214112 (2008).

345. J.H. Rose, J. Ferrante, and J.R. Smith, Phys. Rev. Lett. 47, 675 (1981).

346. J.H. Rose, J.R. Smith, F. Guinea, and J. Ferrante, Phys. Rev. B 29, 2963 (1984).

347. H.R. Schober and P.H. Dederichs, in Landolt-Börnstein Numerical Data and Functional Relationships in Science and Technology New Series 13a K.-H. Hellwege, ed. (SpringerVerlag, Berlin, 1981).

348. V. Keppens, D. Mandrus, B.C. Sales, B.C. Chakoumakos, P. Dai, R. Coldea, M.B. Maple, D.A. Gajewski, E.J. Freemen, and S. Bennington, Nature 395, 876 (1998).

349. J.L. Feldman, D.J. Singh, I.I. Mazin, D. Mandrus, and B.C. Sales, Phys. Rev. B 61, R9209 (2000).

350. W. Schweike, R.P. Hermann, M. Prager, J. Persson, and V. Keppens, Phys. Rev. Lett. 99, 125501 (2007). 
351. http://www.phys.ufl.edu/fermisurface/

352. G. Grimvall, The Electron-Phonon Interaction in Metals (North-Holland, Amsterdam, 1981).

353. H. Chou and S.M. Shapiro, Phys. Rev. B 48, 16088 (1993).

354. I. M. Lifshitz, Zh. Eksp. Teor. Fiz. 33, 1569 (1960). [English translation Sov. Phys. JETP 11, 1130 (1960).

355. Olivier Delaire, Ph.D. Thesis in Materials Science, California Institute of Technology, May $12,2006$.

356. Y.M. Blanter, M.I. Kaganov, A.V. Pantsulaya, A.A. Varlamov, Phys. Repts. 245, 160 (1994).

357. Y. Ding, R. Ahuja, J.F. Shu, P. Chow, W. Luo, H.-K. Mao, Phys. Rev. Lett 98, 085502 (2007).

358. A. Landa, J. Klepeis, P. Soderlind, I. Naumov, O. Velikokhatnyi, L. Vitos, A. Ruban, J. Phys. Chem. Solids, 9-10, 2056 (2006).

359. L. Koci, Y. Ma, A.R. Oganov, P. Souvatzis, and R. Ahuja, Phys. Rev. B. 77, 214101 (2008).

360. L. Dagens, J. Phys. F: Metal Phys. 8, 2093 (1978).

361. B.M. Powell, P. Martel, and A.D.B. Woods, Phys. Rev. B 171, 727 (1968).

362. J.G. Morgan, R.B. Von Dreele, P. Wochner P, and S.M. Shapiro, Phys. Rev. B 54, 812 (1996).

363. S.C. Ng. and B.N. Brockhouse, Solid State Commun. 5, 79 (1967).

364. A.P. Roy and B.N. Brockhouse, Can. J. Phys. 48, 1781 (1970).

365. O. De la Peña-Seaman, R. de Coss, R. Heid, and K.-P. Bohnen Phys. Rev. B 76, 174205 (2007).

366. N.V. Skorodumova, S.I. Simak, I.A. Abrikosov, B. Johansson, and Yu. Kh. Vekilov, Phys. Rev. B 57, 14673 (1998).

367. John S. Tse, Zhiqiang Li, Kentaro Uehara, and Yanming Ma, Phys. Rev. B 69, 132101 (2004).

368. V.V. Struzhkin, et al., Phys. Rev. Lett. 79, 4262 (1997).

369. Z. Li and J.S. Tse, Phys. Rev. Lett. 85, 5130 (2000).

370. G. Steinle-Neumann, L. Stixrude and R.E. Cohen, Phys. Rev. B 63, 054103 (2001).

371. Ying Xu, Lijun Zhang, Tian Cui, Yan Li, Yu Xie, Wen Yu, Yanming Ma, and Guangtian Zou, Phys. Rev. B 76, 214103 (2007).

372. W. Petry, T. Flottmann, A. Heiming, J. Trampenau, M. Alba, and G. Vogl, Phys. Rev. Lett. 61, $722(1988)$.

373. J. Trampenau, W. Petry, A. Heiming, M. Alba, C. Herzig, H.R. Schober, Phys. Rev. B 43, 10963 (1991).

374. G. Grimvall, J. Haglund, and A.F. Guillermet, Phys. Rev. B 47, 15338 (1993).

375. S.Y. Savrasov and D.Y. Savrasov, Phys. Rev. B 54, 16487 (1996).

376. B.N. Harmon and S.K. Sinha, Phys. Rev. B 16, 3919 (1977).

377. O. Delaire and B. Fultz, Phys. Rev. Lett. 97, 245701 (2006).

378. O. Delaire, T. Swan-Wood, and B. Fultz, Phys. Rev. Lett. 93, 185704 (2004).

379. K.-M. Ho, C.-L. Fu, and B. N. Harmon, Phys. Rev. B 29, 1575 (1984).

380. O. Delaire, M.S. Lucas, J.A. Munoz, M. Kresch, and B. Fultz, Phys. Rev. Lett. 101, 105504 (2008).

381. P.B. Allen and J.C.K. Hui, Z. Phys. B 37, 33 (1980).

382. N. Bock, D. Coffey, and D.C. Wallace, Phys. Rev. B 72, 155120 (2005).

383. N. Bock, D.C. Wallace, and D. Coffey, Phys. Rev. B 73, 075114 (2006).

384. N. Bock and D. Coffey, Phys. Rev. B 76, 174513 (2007).

385. L. Koci, Y. Ma, A.R. Oganov, P. Souvatzis, and R. Ahuja, Phys. Rev. B. 77, 214101 (2008).

386. B. Sadigh and V. Ozolins, Phys. Rev. B 57, 2793 (1998).

387. P. Villars, Ed., ASM Alloy Phase Diagrams Center, web service of ASM Int'l, Materials Park, OH, USA, 2007.

388. B. Renker, K.B. Bohnen, R. Heid, D. Ernst, H. Schober, M. Koza, P. Adelmann, P. Schweiss, T. Wolf, Phys. Rev. Lett. 88, 067001 (2002).

389. T. Yildirim, O. Gulseren, J.W. Lynn, C.M. Brown, T.J. Udovic, Q. Huang, N. Rogado, K.A. Regan, M.A. Hayward, J.S. Slusky, T. He, M.K. Haas, P. Khalifah, K. Inumaru, R.J. Cava, Phys. Rev. Lett. 87, 037001 (2001). 
390. M. d'Astuto, M. Calandra, S. Reich, A. Shukla, M. Lazzeri, F. Mauri, J. Karpinski, N.D. Zhigadlo, A. Bossak, and M. Krisch, Phys. Rev. B 75, 174508 (2007).

391. K.-P. Bohnen, R. Heid, and B. Renker, Phys. Rev. Lett. 86, 5771 (2001).

392. X.K. Chen, M.J. Konstantinovic, J.C. Irwin, D.D. Lawrie, J.P. Franck, Phys. Rev. Lett. 87, 157002 (2001).

393. J.W. Quilty, S. Lee, A. Yamamoto, and S. Tajima, Physica C 38, 378 (2002).

394. K.A. Yates, G. Burnell, N.A. Stelmashenko, D.-J. Kang, H.N. Lee, B. Oh, and M.G. Blamire, Phys. Rev. B 68, 220512(R) (2003).

395. A. Cottrell, Introduction to the Theory of Metals (Institute of Metals, London, 1988).

396. L. S. Darken and R. W. Gurry, Physical Chemistry of Metals (McGraw-Hill, New York, 1953), p. 74.

397. A. Hellman, B. Razaznejad, and B.I. Lundqvist, J. Chem. Phys. 130, 4593 (2004).

398. P.H. Hahn, W.G. Schmidt, and F. Bechstedt, Phys. Rev. B 72, 245425 (2005). 
$\mathrm{A}_{2} \mathrm{~B}, 62$

A15, 62, 115

activation barrier, 44

adiabatic

electron-phonon interaction, 109

adiabatic approximation, 42, 93

adiabatic EPI

$\mathrm{P}, \mathrm{c}, \mathrm{T}, 118$

$\mathrm{Ag}-\mathrm{Ni}, 46$

$\mathrm{Ag} / \mathrm{Fe} / \mathrm{Ag}, 82$

$\mathrm{AgBr}, 99$

$\mathrm{AgGaSe}_{2}, 99$

$\mathrm{Al}, 48,104,105$

alloys, 48

$\mathrm{Al}_{2} \mathrm{O}_{3}, 101$

$\mathrm{Al}-\mathrm{Co}, 50$

$\mathrm{Al}-\mathrm{Co}-\mathrm{Ni}, 50$

$\mathrm{Al}-\mathrm{Cu}, 49$

Al-Hf, 50

Al-Li, 23

$\mathrm{Al}-\mathrm{Sc}, 48$

$\mathrm{Al}-\mathrm{Si}, 49$

Al-Ti, 13, 50

$\mathrm{Al}-\mathrm{Zr}, 25,50$

$\mathrm{AlCu}, 51$

alkali halide, 22

AlN-GaN, 64

AlN-InN, 64

aluminides, 52

anharmonic, 29

anharmonicity, 29

cubic, 92

heat capacity, 101

quartic, 92

annihilation, 36

atomic volume, 23, 62

$\mathrm{Au}-\mathrm{Ni}, 46$

$\mathrm{Au}-\mathrm{Pt}, 63$

$\mathrm{Au} / \mathrm{Fe} / \mathrm{Au}, 82$

autocorrelation function, 42

B2, 51,56

$\mathrm{B} 8{ }_{1}, 51$

$\mathrm{Ba}, 105$

Bader algorithm, 114

barostat, 41

bcc, 43, 104

bcc metal, 102, 104, 106

bcc structure, 74

$2 \mathrm{NN}$ forces, 74

binary alloy, 14

binomial expansion, 15
Boltzmann, 1

bond length, 24

bond lengths, 21

bond proportion model, 13

bond stiffness

chemical effects, 25

bond stiffness vs. bond length model, 21

Born approximation, 32

Born-Oppenheimer approximation, 2, 93

Born-von Kármán model, 2, 60, 62

Born-Mayer potential, 46

Born-von Kármán model, 54

Bose-Einstein distribution, 35

boson, 4

boson peak, 81

Bragg-Williams approximation, 16, 18

Bragg-Williams model, 56

Brockhouse, 38

bulk modulus, 27

$\mathrm{C}_{V}, 27$

$\mathrm{C}_{p}, 27$

C-Li, 71

Ca-Sr, 63

$\mathrm{CaF}_{2}, 99$

calorimeter, 31

accuracy, 32

adiabatic, 31

differential scanning, 32

drift, 31

heat pulse, 31

$\mathrm{CaO}, 101$

Car-Parrinello algorithm, 42

carbides, 47

Ce, 30, 84

phases, 84

chaos, 102

charge transfer, 25

chopper spectrometers, 38

classical scattering, 37

cluster expansion, 64

cluster function, 65

Co, 43

$\mathrm{Co} / \mathrm{Fe} / \mathrm{Co}, 82$

composite, 81

computing power, 40

computing resources, 124

conceptual difficulties, 120

configurational coordinates, 1

configurational entropy, 45

nanostructure, 79

constant Q scan, 39 
continuum limit, 81

convolution, 36

Cooper pair, 90

coordinates, 1

coordination number, 14

correlation function

atom, 64

CoSe, 51

Coulomb force, 107

Cr, 30, 110

$2 \mathrm{NN}$ forces, 107

$\mathrm{Cr} / \mathrm{Fe} / \mathrm{Cr}, 82$

creation, 36

critical point

solid state, 84

critical temperature, 13, 14, 17, 43

change with $\Delta S_{\mathrm{vib}}, 13$

ordering, 20, 25, 53

unmixing, 14, 17

CrS, 51

CrSe, 51

CrTe, 51

$\mathrm{Cu}, 105$

$\mathrm{Cu}_{3} \mathrm{Au}, 57$

$\mathrm{Cu}-\mathrm{Fe}, 46$

$\mathrm{Cu} / \mathrm{Fe} / \mathrm{Cu}, 82$

cubic term

symmetry, 97

CuIn, 51

CuSn, 51

CuZn, 56

CuZr, 51

$\mathrm{D}_{22}, 77$

damped harmonic oscillator, 95

Debye model, 11, 26

Debye temperature, 57, 59

Debye temperatures, 12

Debye-Grüneisen approximation, 12

Debye-Waller factor, 34, 52

deformation potential, 94

degrees of freedom

microstructure, 76

density functional theory, 40

deuterium vs. hydrogen, 69

DFT calculations, 123

differential scanning calorimeter, 32

diffusion, 44

entropy, 44

dilation

electron energy, 94

dilute solutions, 45

dimensionality, 81

direct method, 40

disordered systems, 13 dispersions of excitations, 39

dissipative process, 96

$\mathrm{DO}_{3}, 54$

double-differential cross-section, 37

dynamical coordinates, 1

dynamical matrix, 8

Dyson, 13

eigenvalues, 8

eigenvectors, 8

of dynamical matrix, 8

Einstein model, 10, 15, 46, 52, 56

elastic anisotropy, 77

elastic constants, 11,81

elastic energy, 27, 77, 79

elastic scattering, 32

electrochemical measurements, 70

electron jack, 109

electron lifetime, 109

electron-phonon coupling, 87, 104, 112

temperature effect, 116

electron-phonon coupling parameter, 95

electron-phonon interaction, 93, 95, 107

electron-phonon interactions, 30, 88, 89

electron-to-atom ratio, 122

electronegativity, 25, 48, 63, 107, 114, 122

electronic entropy, 28, 86

electrons

independent, 40

strongly-correlated, 40

Eliashberg coupling function, 95

energy, 17

ensemble average, 34

entropy, 17

crystal field, 86

lithiation, 72

magnetic, 76

microstructure, 76

phonon, 3

spin fluctuations, 86

entropy of formation, 60

entropy of mixing

negative, 113

equations of motion, 7

EXAFS, 52

EXELFS, 52

experimental facilities

future, 123, 124

f electrons, 84,88

Faraday, 71

fcc, 43

fcc metal, 102, 104, 105

Fe, 81

nanocrystal, 82 


\section{$\mathrm{Fe}_{3} \mathrm{Al}, 52$ \\ Fe-C, 73 \\ $\mathrm{Fe}-\mathrm{Cr}, 66$ \\ Fe-N, 47 \\ $\mathrm{Fe}-\mathrm{Ni}, 75$ \\ FeCo, 56 \\ Fermi}

chopper, 38

golden rule, 97

level, 111, 115

pseudopotential, 33

resonance, 101

surface, 101, 107

topological transition, 109

surface nesting, 109

surface topology, 108, 119

Fermi surface, 76, 95

pressure, 110

thermal smearing, 119

fermion, 4

fermion field operator, 94

FeS, 51

FeSe, 51

FeSn, 51

FeTe, 51

force constants, 5

four-phonon process, 92

Frölich coupling, 95

free energy

phonon, 3

thermal expansion, 29

Friedel, 75

frozen phonon, 41, 100

fuzzy sphere, 120

GaAs, 23

GaN-InN, 64

$\mathrm{GaP}, 23,101$

Ge, 23, 30

Gibbs, 1

glass, 81

Grüneisen parameter, 22, 24, 29

grain boundary, 79

graphite intercalation, 71

Green's function, 32

Grossularite, 101

harmonic approximation, 4, 6, 29

harmonic model, 2

harmonic oscillator, 3

hcp, 43, 104

heat capacity, 25, 26, 101

quasiharmonic, 27

Helmholtz free energy, 16

Hf, 110 high temperatures importance of, 124

high-temperature limit, 4

hole pocket, 109

honon DOS

interaction, 64

Hume-Rothery rules, 122

hydrides, 69

independent electrons, 40

inelastic scattering, 32

InPt, 51

InSb, 23

instability eigenvector, 74

interaction DOS, 65

interaction partial DOS, 65

interaction phonon DOS, 64

interaction phonon partial entropy, 66

interatomic force constants, 11

intercalation, 70

interchange energy, 14, 45

intermetallic compound, 62

internal stress, 78

interstitial alloys, 68, 73

interstitial $\mathrm{O}$ and $\mathrm{N}, 68$

Invar, 31

irreversibility, 97

Ising lattice, 14

ordering, 18

isolated pockets, 120

K, 105

$\mathrm{KCl}, 101$

kinetic energy, 9, 93

Kohn anomaly, 101, 109

$\mathrm{L}_{2}, 54,59,62$

$\mathrm{Al}_{3} \mathrm{Sc}, 48$

latent heat, 86

lattice dynamics, 5, 6

Laves phase, 49, 62

Lennard-Jones solid, 92

Li, 71

Li battery, 71

Li intercalation, 70

$\mathrm{Li}_{x} \mathrm{CoO}_{2}, 71$

Li-C, 71

Li-H, 69

local mode, 10

long wavelength limit, 11

long-range order, 18

long-wavelength neutrons, 123

Lorentzian function, 83

Lorentzian lineshape, 96

low-symmetry phase, 51

lowering operator, 91 
Lucas, 66

magnetic degrees of freedom, 31, 76

magnon, 76

martensite, 68, 73, 75, 106

mass, 9, 62, 122

metallic radius, 122

$\mathrm{Mg}_{2} \mathrm{SiO}_{4}, 101$

$\mathrm{MgB}_{2}, 118$

$\mathrm{MgO}, 101$

microstructure, 76

miscibility gap, 67

Mo, 30, 100, 102, 105, 109, 110

molecular dyanamics

quantum, 124

molecular dynamics, 41

Monte Carlo simulation, 20

MSRD, 52

multiphonon expansion, 36

multiphonon scattering, 35

$\mathrm{NaCl}, 22$

nanostructure, 79

$\mathrm{Fe}-\mathrm{Ni}, 85$

high-energy modes, 84

low-energy modes, 80

phonon broadening, 83

$\mathrm{Nb}, 109,110$

Nb-Mo, 109, 110

$\mathrm{Nb}-\mathrm{O}, 69$

negative entropy of mixing, 113

negative thermal expansion, 88

neutron scattering, 32

triple axis spectrometer, 123

neutron weighting, 37, 57

neutron-weight

correction, 65

$\mathrm{Ni}, 104$

$\mathrm{Ni}_{2} \mathrm{MnGa}, 76$

$\mathrm{Ni}_{3} \mathrm{Al}, 53$

$\mathrm{Ni}_{3} \mathrm{~V}, 78$

$\mathrm{Ni}-\mathrm{Au}, 62$

Ni-Cr, 62

NiAs structure, 51

NiS, 51

NiTi, 75

nitrides, 47

normal coordinates, 2, 90

NRIXS, 53, 59, 82

number operator, 91

one phonon scattering, 35

partial DOS, 8

partition function, 3

configurational, 15 harmonic oscillator, 3

ordering, 19

$\mathrm{Pd}_{3} \mathrm{Fe}, 62$

$\mathrm{Pd}_{3} \mathrm{~V}, 78$

$\mathrm{Pd}-\mathrm{V}, 23$

$\mathrm{Pd} / \mathrm{Fe} / \mathrm{Pd}, 82$

$\mathrm{PdSb}, 51$

periodic boundary conditions, 7

phase space, 102

phonon

and lattice parameter, 55

branches, 36

damping, 83

density of states, 4

energy broadening, 96, 98

energy shift, 96, 98

entropy, 3

extreme environments, 123

free energy, 3

in multilayers, 83

lifetime, 98

self-consistent theory, 97

thermodynamics, 29

phonon annihilation, 35

phonon creation, 35

phonon dispersion, 92

phonon dispersions, 40, 123

phonon DOS, 4, 8

neutron-weighted, 38

phonon entropy, 27

phonon field operator, 91

phonon partial DOS, 8

phonon propagation, 13

phonon-phonon interaction, 30

phonon-phonon interactions, 89, 90

Planck distribution, 3

plastic deformation, 78

polarization, 7

polarizations, 8

polymorphism, 43

potential energy, 10, 93

pressure, 13, 110

$\mathrm{Pt}_{3} \mathrm{Fe}, 59,62$

$\mathrm{Pu}, 87$

phases, 87

Q information, 123

quality factor, $\mathcal{Q}, 104$

quality factor, $\mathcal{Q}, 83$

quantum field theory

results, 92

quantum oscillator

damping, 97

quasicrystal, 50

quasiharmonic, 29 
reliability of theory, 103

raising operator, 91

Raman spectrometry, 92

Raman spectroscopy, 101, 118

random solid solution, 14

rare gas solid, 99

Rayleigh mode, 80

recoil energy, 36

resonance mode, 10, 59

Rh alloys, 63

scattering

coherent, 34

incoherent, 34

quasielastic, 86

Schrödinger equation, 32

screening, 107, 118

second quantization, 91, 94

self-force constant, 6

shape factor, 34

shape memory alloy, 75

magnetic, 76

Si, 23, 30, 49

single crystals, 123

Sm, 105

small displacements, 6

Sn, 23, 43

soft mode, 74

solid solution, 64

solubility, 44, 45

sphalerite, 23

spin excitations, 1

spin fluctuations, 86

spin variable, 64

spinodal decomposition, 17, 67

square lattice, 18

Stirling approximation, 16

strongly-correlated electrons, 40

structure factor, 34

sublattice, 18

supercell method, 40

superconductivity, 112, 118

superconductor, 90

superionic conductor, 99

switch Hamiltonian, 42

Ta, 102

Ta-N, 69

Taylor series, 6

theory

future, 123

thermal conductivity, 92

thermal electronic excitations, 89

thermal energy, 36

thermal excitations, 108 thermal expansion, 27, 78

free energy, 29

negative, 88

thermodynamics phonon, 29

thermoelectric materials, 107

thermostat, 41

three-phonon process, 92, 95

Ti, 104

time average, 34

timing, 38

titanium compounds, 23

TlBr, 99

topological transition, 109

transition metal, 118

transition metals, 12

twinning, 73

$\mathrm{U}, 78,87$

phases, 87

umklapp process, 92

universal potential curve, 105

unmixing, 62

V, 30, 104, 110

$\mathrm{V}$ alloys, 112

$\mathrm{V}_{3} \mathrm{Co}, 115$

$\mathrm{V}_{3} \mathrm{Ge}, 115$

$\mathrm{V}_{3} \mathrm{Si}, 115$

V-Co, 111

V-D, 69

V-H, 69

$\mathrm{V}-\mathrm{N}, 69$

$\mathrm{V}-\mathrm{Nb}, 111$

$\mathrm{V}-\mathrm{Ni}, 114$

V-O, 69

V-Pd, 114

V-Pt, 18, 111, 114

$\mathrm{V}-\mathrm{X}, 110$

van Hove

singularity, 80

Van Hove correlation function, 35

vanadium compounds, 23

vandalized cage, 59

variational method, 41

vibrational entropy

multiple origins, 121

Vineyard, 44

W, 30, 102

W-Re, 110

waves

phases, 33

wurtzite, 64

$\mathrm{YB}_{6}, 110$ 
Zener, 44, 56

bcc hypothesis, 43

Zn, 110

Zr, 100, 104, 110 